\title{
INTERNET SKILLS
}

VITAL ASSETS IN AN INFORMATION SOCIETY 
Thesis, University of Twente

ISBN: 978-90-365-3086-6

Van Deursen, A.J.A.M. (2010). Internet skills. Vital assets in an information society. Enschede, the Netherlands: University of Twente

Printed by Gildeprint Drukkerijen, Enschede, the Netherlands

People on the cover all granted permission for usage and are actual participants of the studies discussed in this dissertation.

Some of the studies presented in the dissertation were supported by the Ministry of the Interior and Kingdom Relations, the Ministry of Economic Affairs, ECP-EPN, or the IBR Research Institute for Social Sciences and Technology. 


\title{
INTERNET SKILLS
}

\section{VITAL ASSETS IN AN INFORMATION SOCIETY}

\section{PROEFSCHRIFT}

\author{
ter verkrijging van \\ de graad van doctor aan de Universiteit Twente, \\ op gezag van de rector magnificus, \\ prof. dr. H. Brinksma, \\ volgens besluit van het College voor Promoties \\ in het openbaar te verdedigen \\ op vrijdag 17 december 2010 om 16:45
}

door

Alexander Johannes Aloisius Maria van Deursen

geboren op 20 december 1977

te Geldrop 
Dit proefschrift is goedgekeurd door de promotor prof. dr. J.A.G.M. van Dijk en assistent -promotor dr. $\mathbf{O}$. Peters. 


\section{Samenstelling promotiecommissie}

Promotor: $\quad$ Prof. Dr. J.A.G.M. van Dijk

Assistent-promotor: Dr. O. Peters

Leden: $\quad$ Prof. Dr. J. de Haan Sociaal Cultureel Planbureau /

Erasmus Universiteit Rotterdam

Dr. E. Hargittai Northwestern University

Prof. Dr. E. Loos Universiteit van Amsterdam

Prof. Dr. M.J. Steehouder Universiteit Twente

Prof. Dr. Scheerens Universiteit Twente 
Happiness is only real when shared Chris McCandles 


\section{Contents}

1. Introducing Internet Skills 9

$\begin{array}{lll}1.1 & \text { Increasing demands, higher stakes } & 10\end{array}$

1.2 Literacies in contemporary society 14

$\begin{array}{lll}1.3 & \text { Research questions and contents } & 16\end{array}$

1.4 Scope of the dissertation 20

2. Internet Skills and Social Inequality 21

2.1 Introduction 22

2.2 The Internet and social inequality 23

2.3 The digital divide 28

2.4 Internet skills and social inequality 32

2.5 Inequality among whom? 38

2.6 Conclusions 43

3. Defining Internet Skills 45

3.1 Introduction $\quad 46$

3.2 The term "Internet skills" 47

$\begin{array}{lll}3.3 & \text { Overview of related concepts } & 48\end{array}$

3.4 Proposing an Internet skills definition $\quad 58$

$\begin{array}{lll}3.5 & \text { Traditional media skills and digital media skills } & 70\end{array}$

4. Internet Skills Performance Tests 79

$\begin{array}{lll}4.1 & \text { Introduction } & 80\end{array}$

4.2 Method 81

4.3 Results of performance test 1: government information 88

4.4 Results of performance test 2: leisure information 92

4.5 Results of performance test 3: health information 95

4.6 Modeling Internet skills determinants 98

$\begin{array}{ll}4.7 & \text { Evaluating the Internet skills definition } \\ 4.8 & \text { Conclusions }\end{array}$

4.8 Conclusions 111 


\section{Internet Skills-Related Problems}

5.1 Introduction

5.2 Coding Internet skills-related problems

5.3 Identifying Internet skills-related problems

5.4 Conclusions

6. Internet Skills Survey questions

6.1 Introduction

128

6.2 Step 1: Correlations between items and observed skills

6.3 Step 2: Discriminant validity of the Internet skills items

133

6.4 Step 3: Internet skills items in a first-order confirmatory factor analysis

6.5 Conclusions

7. General Conclusions and Discussion

7.1 Introduction

7.2 Level of Internet skills

7.3 Differences in Internet skills

7.4 Internet skills, vital assets in an information society

7.5 Limitations and future research

155

8. Overcoming Internet Skills Divides 159

8.1 Introduction

8.2 Improving new media development

8.3 Improving Internet skills 


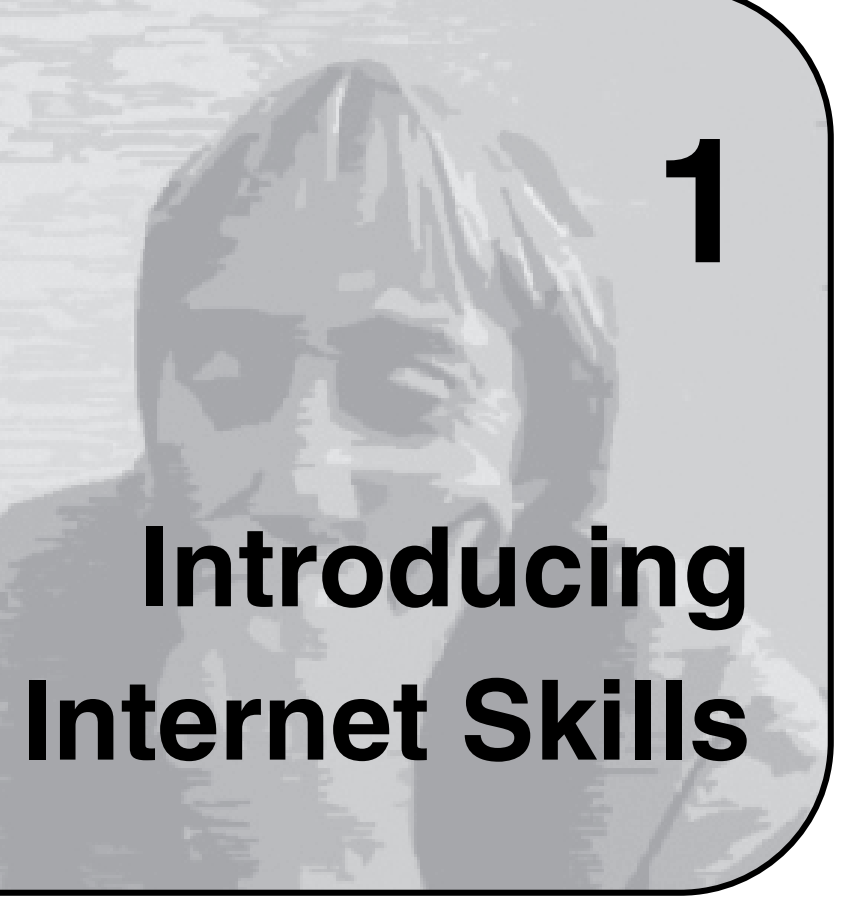

This dissertation is about being able to keep up with the digitalization of contemporary society. It starts with a brief historical overview of changes in communication technologies and the increasing demands they have put on users. Special attention in this chapter is given to the communication technology that in a relatively short period of time has vastly changed the way information is collected and used: the Internet. Since the impact of the Internet depends on the skills that determine the way in which it can be used, these skills are introduced as the main focus of the dissertation. Subsequently, the theoretical and practical goals are discussed followed by the research questions and an overview of the dissertation. 


\subsection{Increasing demands, higher stakes}

While oral traditions did give us the Agricultural Revolution as well as the poetry of Homer, they would not have sufficed for much more than that. Similarly, handwritten works brought us the Renaissance, but printing was needed for the modern era with its more complicated society and therefore greater information needs. To handle the information needs of the future, we will have to use electronic forms of information. (Odlyzko, 1997, p. 3)

Odlyzko refers to the increasing complexity of society, which brings greater information needs. From his quote, it appears that, to some extent, his view corresponds with McLuhan's (1962) idea that major shifts in society's predominant technology of communication are crucial determining forces behind the societal transformations of nomadic, agricultural, industrial, and informationbased cultures. Although it is often discussed whether changes in modes of communication have been decisive for the major shifts of human civilization, these changes do seem to function as important components within these shifts regardless. Investigations of existing oral cultures have shown that a change in the predominant mode of communication, particularly the transition from oral communication to writing, consistently involves a transformation of patterns of community organization, communication styles, and ways of thinking (Boomershine, 1987). This section provides a brief historical overview of societal transformations from a communication technology perspective. The overview reveals that societal change is associated with higher demands being placed on its members.

Humans successfully survived the Ice Age, domesticated plants and animals, settled in villages, and established metallurgy without any transformation in their communication "technology" of the primarily oral transmission of knowledge (Mizrach, 1998). For a long time, oral communication accounted for much of the cultural transmissions from one generation to the next. With the emergence of more complex civilizations, oral communication became deficient, and around 3000 B.C., the Sumerians developed a logographic system for improving recordkeeping and taxation (Mizrach, 1998). This system required years of training to master and was only accessible to elites destined to rule rather than to the majority. Most of the general public required oral communication only and probably did not feel harmed by their lack of reading and writing skills (nor was it 
expected that they possessed them). For centuries, the transmission of culture and work procedures mainly occurred at home or at work. While reading and writing systems improved in the following centuries (e.g., the invention of the alphabet, the addition of vowels to the alphabet, the transition from reading out loud to silent reading), they remained specialist activities limited to a small group of intellectual elite.

The demand for written matter was fueled by the invention and spread of the Gutenberg's printing press in the course of the early sixteenth century (Briggs, 2000). Newspapers, pamphlets, and books were suddenly reproducible at high rates, which influenced the nature of written communication considerably. However, it was several years before printed documents became a method of mass communication. Most people lacked the skills of reading and writing, and relatively large proportions of the population were needed for manual work in agricultural and industrial production (Briggs, 2000). Schooling was needed to increase the number of people that were able to read and write but was far from customary. Around the seventeenth century, Bible-reading, civilization, morals, political obedience, and factory discipline were all cited in arguments in favor of schooling (Stone, 1969). However, schooling remained restricted to the higher classes of society since learning for the poor was considered dangerous; it would encourage them to aspire beyond their status position (Stone, 1969).

During the period referred to as the Enlightenment (an intellectual movement that took place between the 1600s and 1800s), schooling underwent a major transformation. The Enlightenment was characterized by ideas of great value to society, including the centrality of freedom, democracy, and general education (Stone, 1969). As part of the Enlightenment, in the US, Thomas Jefferson was the pioneer advocate for the establishment of public schooling in 1779 based on the belief that a general education was necessary for all citizens (Stone, 1969). It was argued that education distinguishes civilization from barbarism and that the capacity to read makes one society superior to another (Stone, 1969). Although Jefferson's proposal was never realized, in the dawn of the eighteenth century, state-supervised schools appeared in the US and Europe, thereby expanding the reach for formal education and increasing the number of people that attended schools (Rury, 2005). This was an historic step, because until that time, schooling was a privately organized affair in particular churches and in the domestic settings of guilds and small crafts, agriculture, and industry (Rury, 2005). 
Throughout the Industrial Revolution that started in the eighteenth century, agricultural output increased dramatically, adapting to the greater requirements of industrialization. Changes and developments in manufacturing, mining, and transportation affected socio-economic and cultural conditions. Living standards were accelerated by the more rapid pace of technological progress Jefferson, 2005). The organization of schooling by society was further strengthened by the introduction of compulsory education throughout the second half of the nineteenth century. This was another historic step, unique in two ways. First of all, it was now believed that everybody should possess the literacies of reading and writing, both people in cities and rural areas. Secondly, it was the government who told individuals and families what to do with their children in order to improve society by introducing laws that placed restrictions on what families and employers could determine for children. Although the introduction of compulsory education did not pass off smoothly, since education was not nearly as important as manual work (especially in rural areas), in 1959, the United Nation's Declaration on the Rights of the Child declared that children should receive compulsory education, at least in the elementary stages (Harris \& Hodges, 1995).

At the turn of the twentieth century, a host of new media appeared. Besides new telecommunication media, these new media ranged from photography and film to the gramophone, radio, and television. Many of these new media were audiovisual and again required new skills on top of the skills of reading and writing texts. These new skills however, have barely been a part of schooling. This also was the case for telecommunication media. Apparently, the popular view prevailed that everybody is able to speak, hear, and view. Learning to use audio-visual media primarily seemed to be considered a hobby or a matter of professional expertise for a few.

Around the seventies, in many western societies, the number of people working in the blue-collar sectors started to decrease. This trend has carried on since, and in the nineties, only about a seventh of the American working population was actually manufacturing; the rest received, processed, and transmitted information or served the information moguls (Hobart \& Schiffman, 2000). The role of agriculture and industry as the primary economic sectors was replaced by the information sectors, and authorities started to use the term "information society" to emphasize the occurring social changes. There is no universal agreement of 
what exactly constitutes an information society ${ }^{1}$. Moore (1998) pointed out that information is used as an economic resource (organizations make greater use of information to increase their efficiency, stimulate innovation, and increase their effectiveness and competitive position) and that the general public makes greater use of information than before (people use information more intensively in their activities as consumers: to make informed choices between different products, to explore their entitlements to public services, and to take greater control over their own lives). The general consequences of the information society are larger volumes of information, new forms and aggregations of information, and new tools for working with information (Marchionini, 1995).

The information society emerged after the industrial society, first in the shape of print, to be followed by the contemporary electronic era. The concept, however, is often related to electronic forms of communication; "Computer technology is to the information age what mechanization was to the industrial revolution" (Naisbitt, 1984, p. 22). The idea of a whole new type of society was created; although, in fact, all societies discussed are information societies. After all, every form of social cooperation demands the exchange of information. "If there is just more information, then it is hard to understand why anyone should suggest that we have before us something radically new" (Webster, 2002, p. 259). Webster points out that the growth of information had already started in the 1950s; only the mass applications of ICTs that increase the capacity to process information are new. The main point is that besides agricultural products, machines and tools, medical care and social facilities, also information products became important social commodities (De Mul, 2005). Therefore, although the concept of the information society as a revolutionary new type of society is contested, there is no doubt that the importance and the amount of information has spectacularly increased over the last few decades. Information sharing has become the major form of earning a living for large segments of society. This makes coping with the massive amounts of available information and the supporting technologies important for everyone.

\footnotetext{
${ }^{1}$ See also Information Age (Castells, 1996), Computerized Society (Martin \& Norman, 1970), Information Economy (Porat, 1977), and Knowledge Economy (Kim \& Mauborgne, 1999).
} 


\subsection{Literacies in contemporary society}

Communication technology breakthroughs of the last decades can be unified under the name of Information and Communication Technologies (ICTs). They revolutionized the spread of information, and their impact on society is comparable to the birth of writing or the development of the printing press. Important ICT breakthroughs include the inventions of the electronic computer in 1943; the microprocessor, which made computers accessible to the public, in 1971; and the Internet, which originated in the early 1970s when the US Defense Department set up an electronic communication network between computers. "The fundamental principle behind the Internet was that once someone somewhere made available a document, database, graphic, sound, video, or screen at some stage in an interactive dialogue, it should be accessible by anyone, with any type of computer, in any country" (Berners-Lee \& Fischetti, 2000, p. 37). The Internet enables a tremendous decentralization of information and has made it easier than ever for individuals and organizations to publish information to a potentially large audience. After the appearance of graphical browsers in 1993, the diffusion of the Internet occurred with speeds no other media ever reached before. While it took 38 years for the telephone and 17 years for the television to reach a 30\% household diffusion level in the US, the Internet achieved the same rate in only seven years (Katz \& Rice, 2002).

Technology has frequently played a dominant role in defining what skills have been considered important. With the diffusion of ICTs, a new situation emerges requiring additional skills to those of earlier technologies. In contrary to the audiovisual media, computers were considered difficult and reserved for specialists that possessed the necessary advanced new skills. The diffusion of the Internet again requires additional literacies. This can be explained by applying seven communication capacities to the Internet, as proposed by Van Dijk (1999):

- Speed. The Internet allows instantaneous worldwide communication. The amount of available information in a specific period of time is very large compared to traditional media.

- Reach. The potential social and geographic reach of the Internet is worldwide. Although only a small part of the world's population has Internet connectivity, the Internet still allows access to innumerable addresses and contacts. 
- Storage capacity. This is where the Internet stands out most, especially when compared to human memory or print media (Van Dijk, 2001). The massive amount and variety of online information, very different in quality, puts new demands on people who have to use it.

- Selectivity. Users have to choose between Web addresses, menu options, online applications, and information to find that for which they are looking.

- Stimuli richness. The Internet combines text, sound, speech, images, and video. Although this richness supports mental access, it also requires more mental capabilities to process the various stimuli (Van Dijk, 1999).

- Interactivity. Like stimuli richness, interactivity also causes contradictory effects on Internet use. On the one hand, interactivity makes Internet use more attractive, stimulating, immediate, involving, and participatory, but on the other hand, Internet use is more demanding, since it requires many cognitive resources (Bucy \& Newhagen, 2004). Using the Internet requires a more active user compared to traditional media, especially print.

- Complexity. Compared to the more traditional media, the use of the Internet is more complex. Web sites allow for information in which illustrations, graphs, images, and video can be used. Unfortunately, not all Web sites achieve this in a usable manner.

Over the last centuries, the literacies of reading and writing have become increasingly important. With the introduction of compulsory education, the possession of these literacies became organized by society. Now, the use of the Internet further raises the demands put on its users. Therefore, researchers and policy makers are arguing that the technologies of oral communication and the literacy of reading and writing should be accompanied by newer forms of literacy. Just as literacy was a prerequisite for full participation in the industrial society, Internet skills are prerequisites for full participation in contemporary informationbased society. A lack of these skills might result in disadvantages or even exclusion from global literacy communities (Sutherland-Smith, 2002). Unfortunately, in current educational programs, this has not yet fully gotten to the heart of the debate. While compulsory education obliged all children to go to school and learn to read and write, learning to use the Internet is not a standard component of the current curriculum. To the contrary, it is generally believed that technologies such as the Internet empower learners and are additions to learning itself (Buckingham, 
2007). This, however, is dubious, and research on the matter is often misrepresented (Buckingham, 2007).

Many different concepts that relate to new forms of literacy have been adopted. Examples include visual, media, multimedia, computer, ICT, cyber, Internet, network, digital, Web, or information literacy. According to Livingstone, "The spawning of a new literacy as diverse academic disciplines and stakeholder interests join in the debate seems infelicitous" (2003, p. 5). The varied terminologies cause confusion, apparent in three domains (Livingstone, 2003):

- Among the public that require new skills at work, in education, and at home. Often discussed here is so-called computer literacy; however, the exact skills required remain unclear.

- Among policy makers that are responsible for generating an ICT-literate population. Print literacy has long been a central target for educational policies; however, the new forms of literacy are now becoming increasingly important.

- Among the academy where questions of literacy have raised growing attention in the research agenda, drawing together a multidisciplinary mix of specialists in literacy.

Within the different academic disciplines, confusion also seems to exist. The primary term concerned for researchers in information science, for example, is information literacy. Naito (1991, p. 294) argued, "Many people consider information literacy to be the most important skill for the 90's and for the 21st century." However, the term itself appears to be defined "depending on what part of the elephant one is experiencing” (Langford, 1998, p. 65). Information literacy is defined in terms of skills and behaviors. It is not clear whether the term is an umbrella phrase, a new literacy tied to changing technologies, or a basic literacy mirroring the expanding information needs of society (Langford, 1998).

\subsection{Research questions and contents}

The historical overview of the skills that people need to function in society is marked by three epochal tendencies: an expanding number of skills, ever-larger sections of the population that require these skills, and education of these skills that shifts from private to public settings. In this dissertation, the focus is on the 
skills needed to use the Internet. The Internet has become the most dominant digital medium that is linked to all others. Gradually, personal computers have become terminals for Internet use. Digital televisions, telephones, cameras, and videos have also become increasingly connected to the Internet for transmission, reception, and exchange of user-generated content. With the many related concepts proposed, only minor agreement on the exact underlying definition exists. It appears that an explanation of the concept is more important than the term itself. To avoid becoming entangled in terminological disputes and to facilitate a dialogue between the academy and policy makers, in this dissertation, the straightforward term "Internet skills" is used.

The theoretical goals of this dissertation are:

1. To increase the insights into the role Internet skills play in society;

2. To develop an Internet skills definition that can be used in measurements. The practical goals are:

3. To measure the Internet skills levels among the Dutch population;

4. To propose a measurement instrument of Internet skills that can be used in surveys;

5. To provide recommendations for improving Internet skills levels and to improve Web site development from an Internet skills perspective.

To achieve these goals and guide the topic of Internet skills, several research questions are proposed. The first research question is aimed at the role of Internet skills in society. In the former sections, it was argued that the transformation of societies is accompanied by higher demands of its people that increasingly depend on new communication technologies such as the Internet. Higher demands are likely to have societal impacts. Those failing to keep up are in danger of becoming disadvantaged in several realms of contemporary society. One of the most discussed concerns in the area of social inequality is the socalled "digital divide," the gap between people who have and those who do not have access to the Internet. The role of Internet skills in the social inequality and digital divide debate is discussed in Chapter 2.

Research Question 1: How do Internet skills relate to social inequality in contemporary society? 
Before measurements of Internet skills can be conducted, it should be clear exactly what comprises these skills (and why they are termed Internet skills; alternatives might have been "Internet competency" or "Internet literacy"). This requires a comparison of existing related terms and definitions that so far primarily seem to cause confusion. In Chapter 3, an overlapping definition for Internet skills is proposed. This definition goes beyond technical skills by differentiating between both medium- and content-related Internet skills.

\section{Research Question 2: How can Internet skills be defined conceptually?}

The proposed Internet skills definition differentiates between medium- and content-related Internet skills. As will be discussed, the content-related skills, to some extent, correspond with skills that are needed in traditional media. In Chapter 3, differences between skills needed in traditional and new media are discussed.

\section{Research Question 3: What are the similarities and differences between traditional} media skills and digital media skills?

In Chapter 4, it will be argued that performance tests are the most valid way of measuring Internet skills. A series of three performance tests have been conducted among the Dutch population. These three studies focus on a different domain of interest. Since an important goal of the dissertation is to determine the most likely populations to lack Internet skills, several factors that might determine levels of Internet skills are accounted for.

\section{Research Question 4: What is the level of Internet skills among the Dutch population? Research Question 5: Which factors determine Internet skills levels?}

The results of the three performance tests are also used to evaluate the proposed Internet skills definition. In the final part of Chapter 4, the usefulness, reliability, and validity of the definition are discussed. The performance test results are compared with known observations of Internet skills in the Dutch population using methods such as self-assessments and measures of Internet experience. A complete disagreement would raise doubts on the reliability or validity of the framework. 
Research Question 6: What is the reliability and validity of the proposed definition of Internet skills adopted as a particular type of scientific measurement?

A more in-depth analysis of the specific individual Internet skills that users possess is needed to reveal why certain segments of the population have lower levels of Internet skills than others. Therefore, Chapter 5 discusses the results of a qualitative analysis based on recorded screen actions of subjects that participated in the performance tests.

Research Question 7: Which specific individual skills-related problems do users experience online?

Research Question 8: Which factors determine the type and amount of Internet skillsrelated problems experienced?

Measuring Internet skills using large-scale performance tests is both expensive and time consuming. A useful addition to the measurement of Internet skills would be the development of survey questions. In Chapter 6, survey questions are proposed and compared with the results of the actual performance tests. The proposed instrument can be used in future Internet skills measurements.

Research Question 9: Which survey questions are valid and reliable measures of Internet skills?

Chapter 7 provides the overall conclusions and discussion. The consequences of not being able to address the increasing demands are linked to the people who are most likely to possess low levels of Internet skills.

Finally, Chapter 8 proposes several Internet skills-related policy recommendations, both from a supply perspective (Web site development) and from a demand perspective (how Internet skills levels of the population can be improved).

Research Question 10: How can differences in Internet skills be accounted for? 


\subsection{Scope of the dissertation}

The Internet skills considered in this dissertation are skills that everyone should possess in contemporary society. Specific skills (needed, for example, in certain job environments) are not covered. Furthermore, the proposed Internet skills definition is applicable for a large period of time, meaning that specific Internet applications or employed hardware are neglected.

The scope of this dissertation is limited to the Dutch population. Other countries are not included in the measurements, making international comparisons impossible. This being said, there is no reason to expect that the proposed Internet skills definition is not applicable in other countries. The definition professes to be general and cross-border. Evidence should subsequently be provided in other countries. Since measuring Internet skills in performance tests is expensive and time consuming, survey measures for Internet skills are proposed in Chapter 6. These enable easy measurement of Internet skills in other countries. However, they will need further validation and replication outside the Netherlands.

The scope of this dissertation is limited to the information-related part of the Internet. The communication-related part, meaning particular skills required for computer-mediated communication (e.g., using appropriate message length, content, abbreviations, or emoticons in emailing and chatting, social networking, and participation in online discussion), has been neglected. Although the Internet serves more purposes than the provision of information alone, the Internet is virtually synonymous with information location and retrieval (Karchmer, 2001; Tesdell, 2005), and information-related activities remain the most widely used. Of the ten most common activities reported by Internet users in the US, e-mail is the only activity related to communication. Besides fun and shopping, all other items on the list relate to obtaining information (Witte \& Mannon, 2009). Content creation has also been ignored. Only functional skills that are a requirement for typical Internet use, common to the majority of Internet users, are included, making a comparison with functional literacy as a prerequisite for distinguishing the literate from the illiterate possible. Nonetheless, some of the skills defined are also a necessity for communication activities and content creation. 


\subsection{Introduction}

It is impossible to reduce human society to a level. The Socialists may do their utmost, but all striving against nature is vain. There naturally exists among mankind differences of the most important kind; people differ in capacity, skill, health, strength; and unequal fortune is a necessary result of unequal condition. Such inequality is far from being disadvantageous either to individuals or to the community; social and public life can only go on by the help of various kinds of capacity and the playing of many parts, and each man, as a rule, chooses the part which peculiarly fits his case. (Pope Leo XIII, 1891)

Pope Leo XIII believed that inequality is inevitable and has a function in the production of the wealth on which society depends. In his Rerum Novarum, all men are considered equal in regard to their origin and nature and to their rights and duties. Differences of skills and preferences are supposed to be responsible for a world in which no one is equal. The liberal-conservatives similarly argue that social inequality triggers excellence, ambition, and growth. They believe that everyone is responsible for their personal position in society. Contrary views are adhered to by the social-democrats who favor equal chances in personal development and follow the principles of Rawls that are outlined in his Theory of Justice (1993, p. 5-6):

- Each person has an equal claim to a fully adequate scheme of equal basic rights and liberties, which scheme is compatible with the same scheme for all; and in this scheme, the equal basic liberties, and only those liberties, are to be guaranteed their fair value.

- Social and economic inequalities are only acceptable when they satisfy two conditions: first, they are to be attached to positions and offices open to all under conditions of fair equality of opportunity; and second, they are to be to the greatest benefit of the least advantaged members of society.

The suggested inequalities relate to so-called "primary goods," or the basic things people require (Rawls, 1971). Information should be considered one of the primary goods in life (Rawls, 1971). In earlier societies, the functional illiterates were unable to answer the demands of reading and writing, leading to a deprivation of information. In contemporary society, Internet access equates with access to information. Therefore, people who lack the skills needed to use the 
Internet are deprived of information. This is especially true when traditional forms of information are replaced by online versions. Since information should be considered a primary good, unequal access to it is likely to result in social inequality. The main concern of this chapter is to identify the seriousness of this inequality and the role Internet skills play in the inequality debate. From a liberalconservative point of view, an unequal division of Internet skill levels in society is acceptable when this serves the innovation capacity of higher-educated and more productive people. The lower-educated and less productive people do not need a high level of skills for their function in society. Only the existing information-elite should then possess high levels of Internet skills to access online information. The social-democrats would argue that everybody should possess the necessary Internet skills, since this creates the broad informational basis that is required for a functioning society and economy. These contradictory arguments of inequality in contemporary society caused by new technologies are reflected in the so-called digital divide discourse. The digital divide stems from a comparative perspective of relative inequality. The idea behind the concept is that there are benefits associated with ICT usage and that non-usage has negative consequences, causing social inequalities between non-users and users. By applying a digital divide perspective (defined in terms of the Internet as appears to be the usual convention) and by focusing on the impact of Internet skills on one's personal life, in this chapter, it is argued that Internet skills deserve further research attention. To provide an answer to Research Question 2 (How do Internet skills relate to social inequality in contemporary society?), the following topics are subsequently addressed: the relation between the Internet and social inequality, digital divide research and the role of Internet skills, the consequences of having insufficient levels of Internet skills, and the segments of the population that are most likely to lack Internet skills.

\subsection{The Internet and social inequality}

Social inequality appears in many forms, has various causes and consequences, and refers to differences in resources that are unequally distributed (Hoffman, 2008). In order to count as social inequality, the unequal distribution of these resources must be the systematic result of a particular social process (Hoffman, 2008). An unequal distribution that occurs regularly between the same social segments is perceived as injustice (Hradil, 2001). A classification of resources is suggested by 
Bourdieu (1984) who elaborated on the ideas of Marx and Weber. Both sociologists explored the origins and functions of social inequality in the industrial society. Marx distinguished between societal classes that are determined by their economic position; the main classes are the capitalists or the bourgeoisie that own and control the means of production and the working class or proletariat that can only sell its labor power (Bilton et al., 1996). Although, according to Marx, technology and economic activity primarily determine the shape and form of human relationships, he also attributes social development to more than simply the forces of production (Sabel \& Zeitlin, 1985). This idea was further developed by Weber who considers human society highly stratified or multi-layered and made up of individuals that are all attempting to expand their sphere of control over various social resources (Holborn \& Langley, 2004). Social resources can be anything that is socially desirable and limited in supply; they include the means of production and property, but also other less tangible entities such as social respect, physical strength, or intellectual knowledge (Hoffman, 2008). Weber defined classes by their life chances and opportunities of which the latter can be described by wealth and prestige (Hoffman, 2008).

Bourdieu also believed that society cannot be analyzed simply in terms of economic classes. He reintegrated both Marx's and Weber's ideas by defining economic, cultural, and social capital. The term "capital" was used to highlight differences between societal groups (Hoffman, 2008). The unequal distribution of capital forms the basis of its effect, namely making profits and instituting rules favorable to capital reproduction (Hoffman, 2008). Economic capital reflects Marx's ideas, including both monetary and property assets (that can be converted directly into money), but also other economic possessions that might increase one's capacities (Hoffman, 2008). Social capital consists of resources based on group membership, relationships, networks, and support. It "constitutes the totality of current and potential resources connected to a durable network of more or less institutionalized relations characterized by mutual knowing or acknowledgements; in other words, social capital is a resource based on the affiliation to a group" (Bourdieu, 1984, p. 190). Cultural capital is formed by the knowledge, skills, education, and advantages that give a person a higher status in society (e.g., titles or degrees). Later, Bourdieu added symbolic capital, resources available to an individual based on honor, prestige, or recognition. The forms of capital play an important role in the enduring struggle between the dominating and the dominated classes (Lawley, 1994). It is through the acquisition of capital 
and the use of symbolic capital to perpetrate symbolic violence that classes guarantee their own legitimacy and reproduction (Lawley, 1994).

Since all forms of capital can promote inequality, the question arises how the Internet contributes to these forms of inequality. Tambini (2000) argues that the non-hierarchical nature of the Internet together with the declining cost of computing technologies and increasing user-friendliness are socially leveling and undermine existing patterns of class, race, and gender inequalities. To the contrary, Witte and Mannon (2009) argue that the Internet is both intertwined with and consequential for inequality. They consider the Internet paradoxical, since it is both an emblem of a free and open society, but also an active reproducer and possible accelerator of social inequality. Max Weber (1978, p. 938) argues, "Every technological repercussion and economic transformation threatens stratification by status and pushes the class situation into the foreground." It appears that all forms of capital defined by Bourdieu are applicable to the Internet. In fact, Internet use and the forms of capital seem to reinforce each other (Van Dijk, 2005). First of all, all forms of capital affect Internet access. Economic capital is a requisite to have the supporting means (e.g., a personal computer and a subscription to an Internet provider). Social capital is needed to learn to connect to and use the Internet with the help of others and to reach others. Cultural capital is needed to cope with the diverse amount of available content supplied for people with different cultural backgrounds. When these three requisites are met, the Internet in return affects the three forms of capital (at least, for those having the skills to use it). Economic capital can, for example, be increased by buying profitable resources online or by finding better jobs. Social capital can be increased by extending physical networks to virtual ones, increasing civic engagement, and increasing sense of community (Katz \& Rice, 2002). Finally, cultural capital can be increased by using the Internet, for example, for learning purposes. The result is that some individuals use the Internet in capital-enhancing ways, while others either do not use the Internet at all or utilize it in less effective and less profitable ways or simply for leisure (Hargittai \& Shafer, 2006; Zillien \& Hargittai, 2009). The suggested forms of capital are affected by Internet use in such a way that those with a higher status are advantaged by using online information to increase their forms of capital. Furthermore, it is demonstrated that the intensive and extensive nature of Internet use among the well-off and well-educated suggests an elite life-style from which those with less capital are marginalized (Van Dijk, 2005; Witte \& Mannon, 2009). The result is that groups 
with fewer forms of capital are likely to be persuaded in negative ways; flights will be booked, concerts will be sold out, jobs will be given away, and dates will primarily be granted to those having access. The communication capacities of the new media discussed in Chapter 1 (e.g., reach, selectivity, and interactivity) evidently are effective on these occasions. The initial use of the Internet is typically restricted to those at the top of existing social hierarchies. It functions as a commodity for which the distribution-at least initially-tends to follow existing divisions of class, race, and gender (Selwyn, 2006; Van Dijk, 2005; Willis $\&$ Tranter, 2006). Rather than equalization, the Internet tends to reinforce social inequality and lead to the formation of disadvantaged and excluded individuals (Golding, 1996; Van Dijk, 2005). Witte and Mannon (2009) furthermore illustrated that Internet access should be understood as an asset to maintain class privilege and power and that capitalist relations of production are maintained since the inequalities upon which they rest are reproduced from one generation to the next.

The Internet is one in a long series of information and communications technologies-from speech, to printing, movable type, telegraphy, telephony, radio, and television-that arguably influenced patterns of social inequality by destroying existing competencies and permitting early adopters to interact with more people and acquire more information over greater distances in a shorter period of time (DiMaggio, Hargittai, Celeste, \& Shafer, 2004). In contemporary society, the new patterns of social inequality even seem to go beyond legitimate forms of inequality. Where the liberal conservatives and Leo XIII would argue that inequalities serve society as a whole, it is more likely that the Internet structurally rewards and benefits a specific group of people that has access to information and services that are most relevant to them. Mason and Hacker (2003) support the notion that the Internet serves to increase social inequalities by applying Adaptive Structuration Theory, which holds that society and its rules and resources are reproduced when members' actions reinforce the systems they have created or that existed prior to the use of a communication technology. Mason and Hacker argue that there is a "duality of structure" inherent in this, as the rules and resources both affect and are the outcome of the interaction. The following has been proposed by Mason and Hacker (2003):

Appropriation occurs when rules and resources are taken from a larger social context, as when a group takes on rules from the 
larger organization. When interacting via IT, users appropriate a society's rules and resources, and they utilize the technology in accordance with them. Essentially, technology is used in ways that work to increase the resources of the users. (p. 48)

According to Mason and Hacker (2003), this interaction is comparable to the mutual relationship between the Internet and different forms of capital as discussed earlier:

Both the structure of IT and the appropriated social rules and resources result in the reproduction of the roles, rules, and resources that exist outside of the technology. Those with power and resources outside of the IT context are the primary early adopters of the technology. They use the technology to meet their needs, and the resources and rules they brought into the IT context initially served to shape the roles and rules of those interacting via IT. This serves to reproduce the existing power relations in the social system and even strengthens them, as it opens up a new channel from which those without power and resources are further excluded. This suggests that the rapid evolution of IT, which meets the increasing demand for the more sophisticated and efficient processing of information determined to be valuable by those who negotiate what has value in society, has ensured that those already possessing sophisticated resources and skills continue to shape the technology. The outcome is a technology that primarily meets the needs of those who adopted it first, and the unintended consequence of this is that those already excluded fall further behind. (p. 48)

Other suggestions that the Internet increases social inequalities can be found in theoretical work on the network society. According to Castells (2004), networks constitute the new social morphology of society. He defines the network society as "a society whose social structure is made of networks powered by microelectronics-based information and communication technologies" (p. 3). One of the major axes of social inequality is the divide between those who are networked and those who are not (Castells, 1996). Castells (1999) argues that networks are characterized by a space of flows that overwhelms and pervades the traditional spaces of places. He contends that the particular framing of ICTs in the context of global, informational, and increasingly de-regulated capitalism has been a major factor in the increase of inequality and, particularly, of social exclusion. Because technological access is essential for improvement of living conditions and 
personal development, ICTs deepen discrimination and inequality in the absence of deliberate, corrective policies (Castells, 1999). Van Dijk (2006) defines the "network society" as a form of society that organizes its relationships in media networks that gradually replace or complement the social networks of face-to-face communication. For Van Dijk, networks have become the nervous system of society and are shaping the prime mode of organization and most important structures of modern society. In a network society, information should be considered a positional good, and some positions in society create better opportunities than others for gathering, processing, and using valuable information (Van Dijk, 2006). The positions people have in networks determine their potential power, which reflects the ideas of Weber who argued that human relationships should be added to economic assets when discussing social inequality. When someone in a network society has only a marginal position or no position at all, this entails social exclusion. Those who are very much included because they do have a central position increase their power, capital, and resources (Van Dijk, 2006). With unequal positions comes an unequal distribution of positional goods. The appropriation of these goods determines who is first in the acquisition of particular facilities and gains in networks, the mechanism of opportunity hoarding (Tilly, 1999). In opportunity hoarding, those included exclude people on the opposite side of the boundary from use of a valueproducing resource (e.g., the Internet). They capture the returns and devote some of the returns to reproducing the boundary (Tilly, 1999).

The outline above suggests that the Internet has the potential to strengthen traditional sorts of inequality rather than ameliorate it. It is also likely that these new forms of inequality seem to go beyond legitimate forms of inequality. The difference between legitimate (e.g., income differences between persons with different qualification levels) and illegitimate inequality (e.g., income differences between men and women with the same qualification level) (Hoffman, 2008) gets to the heart of what social scientists call the "digital divide," or the gap between those with access to the Internet and those without.

\subsection{The digital divide}

Original conceptualizations of the digital divide were rather superficial; they only considered binary classification of physical access to the Internet, thereby primarily 
identifying Internet access as a result of differences in capital; you either had the resources to establish a connection to the Internet or you did not. Having a connection meant being on the positive side of the debate and more or less assumed to be having access to all the advantages the Internet had to offer. The singular connotation of having physical access or not having access, for example, seems disseminated by Compaine (2001) who considers the digital divide to be "a nonexistent myth" and a "luxury" problem. He rests his beliefs on the diffusion of innovations theory of Rogers (1995) who theorized that innovations would spread through a society in an S-curve. The S-curve describes the relative speed with which members of a social system adopt an innovation, measured by the length of time required for a certain percentage of the members of a social system to adopt an innovation (Rogers, 1995). The adoption rate contains a point in time, called critical mass, when enough individuals have adopted an innovation in order that the continued adoption of the innovation is self-sustaining (Markus, 1987). From this point of view, the digital divide should steadily disappear as the diffusion rate reaches saturation. The gradual process of the diffusion of a technology from the higher social strata to the lower strata is referred to as the "trickle-down" effect (Norris, 2001; Van Dijk, 2005; Willis \& Tranter, 2006).

By comparing the diffusion of television, radio, and telephone with the diffusion of the Internet, Compaine (2001) stressed that eventually Internet access automatically reaches high levels of saturation due to market competition, technological developments, and the promotion of technologies in government policies. This would suggest that the digital divide should be considered a temporary problem since physical access to the Internet has been constantly growing since its appearance. The presentation of the digital divide as a temporary problem of physical access has furthermore been accelerated by the migration of the Internet to platforms such as digital television and mobile phones (Golding \& Murdock, 2001).

Although the diffusion of innovations theory has been applied to the study of Internet diffusion (e.g., Forman, 2005), there are serious problems with this theory when applied to the Internet (Norris, 2001; Van Dijk \& Hacker, 2003). Norris (2001) elaborated on the diffusion of innovation theory and distinguished between normalization and stratification models. The normalization model, supported by Compaine, maintains that Internet access will eventually reach full saturation among households and thus results in a disappearance of the digital divide. To the 
contrary, the stratification model maintains that people who adopt a new technology first will not stop obtaining new ones, suggesting an ever-lasting digital divide. There are different points of departure and arrival for the higher and lower social strata, as some strata may never reach full access (Norris, 2001). The notion that this theory was originally focused on traditional mass media (e.g., the telephone and television) should be added to the criticism of applying diffusion of innovations to the Internet. As outlined in the first chapter, the Internet differs from these media in several aspects. When the diffusion process is carefully evaluated, the adoption decision unfolds as a series of stages (Rogers, 1995): knowledge, persuasion, decision, implementation, and confirmation. It is the first stage that troubles the application of diffusion of innovations to the Internet. This stage includes the possession of information that is necessary to use the technology (Rogers, 1995). This stage might only be marginally important in the use of more traditional media that are relatively easy to use. The Internet, however, as we will see later, has communication capacities that require new skills, causing serious barriers in the diffusion process.

The importance of Internet skills in the social inequality and digital divide debate becomes even more apparent when studying the knowledge gap hypothesis. This theory of media effects mainly considers traditional media, but is often seen as a forerunner to the digital divide concept. Tichenor, Donohue, and Olien (1970) suggested that when the infusion of mass media information into a social system increases, segments of the population with higher socio-economic statuses tend to acquire this information at a faster rate than the lower-status segments. The basic assumption of the knowledge gap hypothesis combines the components of information (with the value judgment that more information is better), media, and two or more groups of receivers that can be classified into "high" and "low" groups that are positively correlated (Bunz, 2009). Bunz applied this basic logic to the digital divide and argued that instead of information or knowledge, the digital divide literature now refers to literacy, skills, or ability with the assumption that more literacy or skills is better for the individual. Thus, while the original knowledge-gap hypothesis suggested that differences in socio-economic status are responsible for the acquisition of knowledge, the skills that influence both the amount of information and the kind of information retrieved online are now also suggested as an explanation (Bunz, 2009). The use of the traditional mass mediaon which the knowledge gap focuses-was relatively easy compared to the Internet. Use of the Internet requires a broad range of skills enabling navigation 
through a vast amount of information rather than simply reading newspapers or watching television. Relative to print media and television, Internet usage requires not only enabling technologies, but also users with sufficient skills to use the Internet (Bonfadelli, 2002). This is further discussed in Chapter 3.

The discussion above stresses that the digital divide should not be considered a divide in physical access only. This limitation is recognized by more recent theories of Internet adoption and use (Freese, Rivas, \& Hargittai, 2006). The following has been proposed by Jung, Qui, and Kim (2001):

When exclusive emphasis is placed on owning or having access by using these have/have-not comparisons, the assumption is that either all haves will incorporate the technology in their everyday lives in the same manner and to the same degree or that the difference in the quality of Internet connection among the haves is unimportant. (p. 509)

An increasing number of researchers have argued that more attention should be paid to social, psychological, and cultural backgrounds (Van Dijk, 2006), resulting in several conceptualizations of the digital divide:

- Kling (2000) suggested a distinction between technical access (material availability) and social access (the professional knowledge and technical skills necessary to benefit from information technologies). Similar distinctions have been suggested by Hargittai (2002) under the terms firstand second-level digital divide and Attewell (2001) between first digital divide and second digital divide.

- DiMaggio and Hargittai (2001) suggested five dimensions along which divides may exist: Technical means (software, hardware, and connectivity quality), the autonomy of use (location of access and freedom to use the medium for one's preferred activities), use patterns (types of uses of the Internet), social support networks (availability of others one can turn to for assistance with use and the size of networks to encourage use), and skill (one's ability to use the medium effectively).

- Warschauer (2003) argued that besides the physical access to ICT, other factors such as content, language, literacy, educational level attained, and institutional structure must be considered as well in the evaluation of the level of availability and usage of ICT in the society. 
- Mossberger, Tolbert, and Stansbury (2003) distinguished between physical access (access divide); the possession of ICT knowledge and skills (skills divide); using ICT for finding new jobs, use at work, and constant learning (economic opportunity divide); and the possibility to use ICT in democratic processes (democratic divide).

- Van Dijk (2005) proposed a causal model with four types of access to ICTs: Motivational access (the lack of the elementary digital experience by people who have no interest or feel hostile towards ICTs), physical access (the availability of ICTs), digital skills (the ability to use ICTs), and usage access (the opportunity and practice of using ICTs).

- Wilson (2006) considered eight aspects of the digital divide: physical access (access to ICT devices), financial access (cost of ICT services relative to annual income), cognitive access (ICT skills), design access (usability), content access (availability of relevant applications and information online), production access (capacity to produce one's own content), institutional access (availability of institutions that enable access), and political access (access to the institutions where the rules of the game are written).

These scholars stress the cultural, educational, political, and socio-economic aspects of the digital divide and believe that while gaps in physical access are being addressed, other gaps seem to be widening. Therefore, the attention in the digital divide discourse has now shifted to other types of inequalities (e.g., inequalities in skills). Although Internet skills are relatively new in the debate, it appears that they are becoming increasingly important. After all, people need an adequate level of skills in order to become effective Internet users (Eastin \& LaRose, 2000).

\subsection{Internet skills and social inequality}

Thus far, it has been substantiated that the Internet plays an important role in the social inequality debate. Furthermore, it is argued that in the digital divide discourse, the attention has shifted from physical access to differences in Internet skills, making differences in Internet skills a possible contributor to social inequality. In discussions about social inequality, the specific conditions one has to meet are usually not separately discussed. Therefore, there is little generalized understanding of the ways in which Internet skills divides match the more conventional patterns of social inequality. From the former discussions, however, 
it appears that Internet skills play a significant part. The following has been proposed by Castells (2001, as cited in Livingstone, 2003):

Increasingly, as computer use is ever less a lifestyle option, ever more an everyday necessity, inability to use computers or find information on the web is a matter of stigma, of social exclusion; revealing not only changing social norms but also the growing centrality of computers to work, education, and politics. (p. 6)

Although the old media still offer basic entries to most information and services, those who have the skills to use the Internet tend to come first on the labor market, in political competitions, and in social and cultural affairs. The relation between social inequality and Internet skills is further investigated in this section. Specific focus is given to individual life chances. An effort to interpret the concept of social inequality in individual life chances demands a specification of the types of inequality that are at stake. In the literature, systematic descriptions of inequality often consist of categories of the most valued resources in society, for example, the forms of capital suggested by Bourdieu. Added to the forms of economic, social, and cultural capital are health and political capital, two domains in which the Internet plays an important role. This categorization guides the societal purposes that may be fulfilled through the possession of Internet skills. From the overview below, it will become clear that the stakes of not being able to work with the Internet are high. They are nothing less than participation or exclusion from contemporary and future information-based society. These skills appear to be vital assets.

\subsubsection{Economic inequality}

"The Digital Divide may have serious economic consequences for disadvantaged minority groups as information technology skills become increasingly important in the labor market" (Fairlie, 2004). Workers who have the skills to use the Internet effectively may perform better than those who have not (they have improved access to information, faster and more efficient communication, greater access to learning opportunities, or higher job satisfaction leading to greater job commitment) and therefore have privileged access to desirable jobs and/or obtain more generous rewards for their performance (DiMaggio \& Bonikowski, 2008). Workers that are less skilled with new technologies have suffered the costs of economic change as wage inequality has expanded since the late 1970s (Goldin \& 
Katz, 2008; Mossberger, Johns, \& King, 2006). Goss and Phillips (2002) found that, in the manufacturing sector, Internet users were paid a wage premium of $13.5 \%$ in 1998 (after controlling for other factors influencing pay). Freeman (2002) similarly found a 14\% premium in 2001 (controlling for computer use). DiMaggio and Bonikowski (2008) revealed that skills and behaviors associated with Internet use are rewarded by the labor market and that inequality in mastery of technology is a valid concern for students of social stratification. Besides higher income and better opportunities in the labor market, Internet skills also offer other economic opportunities (e.g., when buying products, trading stocks, conducting price comparisons, or enforcing low prices by uniting with others).

\subsubsection{Social inequality}

Bourdieu's definition of social capital stresses the importance of social connections as a means of obtaining resources. The Internet intensifies the interpersonal transformation from door-to-door to individualized place-to-place and person-to-person networks (Wellman, 2001). The Internet enables the expansion and maintenance of social relationships established with people from around the world and increases social capital in terms of social contact, civic engagement, and sense of community (Katz \& Rice, 2002; Quan-Haase, Wellman, Witte, \& Hampton, 2002). However, no clear patterns have emerged. Some argue that the Internet enables individualism by taking people away from face-to-face and phone contact (e.g., Slouka, 1995). Others claim that the Internet provides new and better ways to communicate and consider the Internet primarily a convenient extension of offline activities and as an additional means of communication beyond traditional contact (Müller, 1999). Katz and Aspden (1997) consider the Internet a medium in which Internet skills appear to be the most important determinant of friendship formation. Similarly, Katz and Rice (2002, p. 326) argue, "The Internet complements and even strengthens offline interactions, provides frequent uses for social interaction and extends communication with family and friends." Regarding seniors, the variety of online information provides a new connection to the outside world, improving their social communication and well-being (Nahm \& Resnick, 2001). The elderly who are trained to use the Internet report having high levels of social connectivity, high levels of perceived social support, and generally more positive attitudes toward 
aging (Cody, Dunn, Hoppin, \& Wendt, 1999). Positive effects, however, depend on the possession of the required Internet skills.

Other social benefits of high levels of Internet skills might be found in social networking. Supporting research, however, is scarce. Valkenburg, Peter, and Schouten (2006) found that using social network sites increases the frequency of interaction with friends, which, in turn, results in positive effects on self-esteem and, in due course, on satisfaction with life. Thus, people that do not have the skills to create and maintain an online profile will eventually receive relative disadvantages compared to those who do. The design of an attractive and informative personal profile requires advanced Internet skills. One should know how to present oneself in these profiles with textual and visual aids to attract new visitors, and to satisfy existing contacts. This applies even more to online dating. Those participating in social networking sites reveal very different levels of the necessary skills (Boyd \& Ellison, 2007; Knobel \& Lankshear, 2008). It is no exaggeration to claim that Internet skills will decide success or failure in all future Internet markets aimed at building social relationships.

\subsubsection{Health inequality}

The use of the Internet for information and services in the domain of healthcare is growing rapidly (Freese et al., 2006). Eysenbach and Köhler (2003) calculated that about $4.5 \%$ of all Internet searches worldwide are health-related. In 2002, 62\% of Internet users in the US had gone online in search of health information (Pew Internet Project, 2002). Health-related Web sites are often used to search for health information, Internet-based peer support groups, online health consultations, and the delivery of health interventions (Griffiths, Lindenmeyer, Powell, Lowe, \& Thorogood, 2006). In the search for online health information, information about a particular illness or condition is most popular, and people seem very proactive in the use of this information (Fox \& Rainie, 2002). People perform search operations after being prescribed a new medication or course of treatment, because they are dealing with an ongoing medical condition, because they have unanswered questions after a doctor's visit, or because they have decided to change their diet or exercise habits (Fox \& Rainie, 2002). The healthrelated searches are not restricted to people's own information needs. They also involve caretaking of the family (Fox \& Fallows, 2003). Providing health 
information and services over the Internet has many potential benefits for its users. They include saving time, saving effort, easier access, getting help when feeling embarrassed or stigmatized, and having the ability to obtain extra information (Griffiths et al., 2006). Other potential benefits include healthier lifestyles, early detection of potential medical problems, collaborative treatment of illnesses, and access to treatments to which a local provider may not have access (Mittman \& Cain, 1999).

Besides the benefits of the online provision of health information and services, several drawbacks can only be compensated for with high levels of Internet skills. First, very few tools are available to help people find relevant information in the excessive amount that is available (Benigeri \& Pluye, 2003). Second, the scientific quality of online health information is difficult to evaluate (Pandolfini, Impicciatore, \& Bonati, 2000; Shepperd, Charnock, \& Gann, 1999). Third, the available information is often incomplete (Benigeri \& Pluye, 2003). Fourth, users must be able to understand the information found and put it into practice (D’Alessandro, Kingsley, \& Johnson-West, 2001). At present, most medical information Web sites present technical information to a population that is unfamiliar with medical literature. In addition, difficulties with technical terms and the reading skill level required are also problematic (Benigeri \& Pluye, 2003). These drawbacks exclude many people from accessing online medical information. Distressing is the fact that the information and assistance provided by the Internet is in practice unavailable precisely to those citizens who have the most frequent health problems and the highest mortality rates: people with low levels of education and the elderly. For them, the lack of skills can even become vital in the most literal sense. This happens when the unskilled cannot find the hospital with the shortest waiting list for surgery or the best qualifications. This also occurs when they lack any other crucial information that helps them in preventing or relieving an urgent disease or when they are not able to ask for a second opinion about a proposed treatment.

\subsubsection{Political inequality}

In the 1980s, the term "New Public Management" was introduced. The focus of this management approach was on the relationship between a government and its citizens. The latter were no longer seen as subjects of the state, but as clients. In 
New Public Management, the way in which governments influenced their citizens shifted from reinforcement of the law towards service provision. Triggered by the dot.com hype-in which the Internet was considered a holy grail for service delivery_ - and by positive Internet access figures, the Internet now plays a major role in the service provision by many public authorities (Van Deursen \& Pieterson, 2006). Unfortunately, the policy to offer an increasing number of services and information online is characterized by barely funded presuppositions of what citizens want and of the citizens' skills when using the Internet (Van Deursen, 2007; Van Dijk \& Ebbers, 2006). Some of the applications are offered as an alternative to their traditional counterparts (e.g., service desks or telephones), making them unavailable to those with insufficient levels of Internet skills. The problem of being short of skills becomes urgent when governments suppose that citizens are able to complete virtually every task on the Internet. Policy advisors often suppose that the Internet is a generally accessible channel for both informing and communicating with citizens (Van Deursen, 2007). Although there are various policies that attempt to resolve particular aspects of the digital divide, they seem to consider the problem of the lack of connectivity and participation as temporary, since the present, mainly elderly, generation of computer illiterates will soon become extinct (Loos, 2010; Van Deursen, 2007). There is a real danger that some people will be deprived of these services, introducing discrimination that infringes fundamental principles of today's democracies (Servaes, 2003).

Similar conclusions can be drawn when considering political participation. Tranter and Willis (2002) argue that the Internet may serve to alter existing political organization by engaging social groups not engaged in previous forms of civic participation. However, contrary to popular expectations in the 1990s, the Internet is not drawing more people into the political process (Bimber, 2003; Brundidge \& Rice, 2009; Katz \& Rice, 2002; Quan-Haase et al., 2002; Scheufele \& Nisbet, 2002). Besides the fact that technical opportunities do not automatically fix basic problems of a lack of motivation, a lack of Internet skills should also be considered an important cause. Online political information retrieval meets the same problems as searching for online health information. Online discussion channels and decision-making tools require a high level of Internet skills. Giving even more power and influence to the people that are politically motivated and that have the necessary skills may result in even stronger inequalities. 


\subsubsection{Cultural inequality}

The Internet provides an enormous and diverse supply of leisure- and entertainment-related content often freely available to those with the skills to access them. Among the possibilities are game playing, listening and sharing music (e.g., iTunes), watching videos (e.g., YouTube), using forums for collectively practicing hobbies, and sharing and trading cultural objects such as pictures, stories, and drawings. Think of the many leisure (such as hiking) and cultural (such as museums) organizations that are present on the Internet. Jones Thompson (2005) reports that sites associated with music, games, movies, videos, pornography, and gambling or sweepstakes receive the heaviest Internet traffic. In the tourism domain, planning and booking holidays on the Internet is becoming a worldwide trend and is the most important industry in terms of electronic commerce volume (Susskind, Bonn, \& Dev, 2003). Tourism is no longer limited to brochures, print, or TV advertisements. It now includes an online community of tourism opportunities and information. Of course, the benefits of online tourism-such as greater availability of trips, price comparisons, and quality assessments - are only available to those who possess the skills to use the information and services online. The same applies to using the Internet for educational purposes. The Internet also provides opportunities to obtain an education via distance learning. This implies that those who have the skills to use the Internet might strengthen their educational advantage over those who do not have these skills.

\subsection{Inequality among whom?}

Since digital exclusion is strongly associated with traditional forms of social exclusion-for example by socio-economic status, region, and deprivation (Norris, 2001)—differences between several demographic groups with regard to Internet skills can be suggested. Even as there is some variation to the scale of difference, the segments of the population that are most likely to be excluded are most commonly defined in terms of gender, age, education, income, race, geography, and disability. Specifically relevant here is to what extent returns from Internet skills vary for different types of users. 


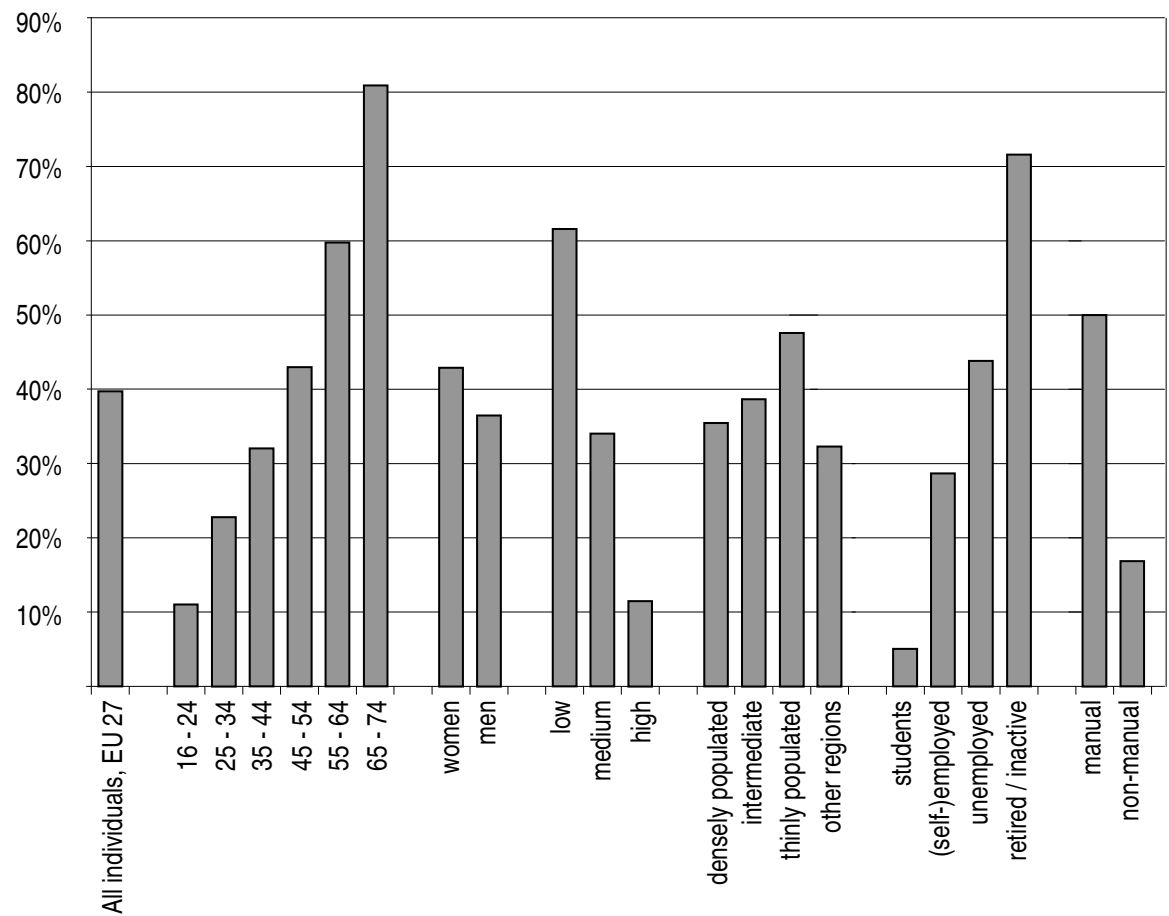

age

gender education location

employment

Figure 2.1 Individuals Lacking Internet Skills

Source: Eurostat Community Survey on ICT Usage by Households and by Individuals between 1674 Years Old (2007). EU27 without Malta.

Most of the large-scale surveys that have been conducted reveal large differences in Internet skills among segments within populations or among countries with high Internet diffusion. However, these studies mainly use self-reports that are not very valid measurements for the actual level of Internet skills (see Section 4.2). Furthermore, in most cases, only the command of hardware and software is considered. To get some indication about the level of Internet skills, data provided by the European Commission are available. Figure 2.1 contains proportions of people in the European Union with no Internet skills by socio-economic status in the year 2007. These skills, however, are measured by asking respondents which of a number of possible activities they have ever carried out. There is no estimation, measurement, or observation of actual Internet skills levels. The provided figures are therefore more an indication of the type of use than of the skills that a person has or, in this case, lacks. The figure shows that specific groups of citizens barely have the necessary skills to use the Internet. In the US, indications for low levels 
of Internet skills are provided by Katz and Rice (2002). They identified a substantial group of people who had used the Internet for (typically) a short while and then stopped using it. In fact, this accounted for one fifth of all respondents as of the time they conducted their surveys. The most frequently cited reasons for dropping out were problems with physical access and the Internet being too hard to master or too complex.

The most valid scientific instrument to measure Internet skills is a performance test in a controlled environment (Hargittai, 2005; Kuhlemeier \& Hemker, 2007; Pask \& Saunders, 2004). Hargittai (2002) provided 54 demographically diverse American experimental subjects with five rather different Internet search tasks. The results revealed enormous differences in the accomplishment of these tasks and the time needed for them (from a few seconds to more than 15 minutes). Respondents searched for content in myriad ways, and there was a large variance in whether they were able to find various types of information and in how long they took to do so. Most of the tests, however, are conducted by scholars of library and information science. These studies tend to focus on the technicalities of searches, such as the number of search terms used in a search query, and are mainly conducted among people who are motivated to follow computer courses, people from particular academic communities, or among college students, which makes it impossible to generalize the findings to the broader Internet user population (Hargittai, 2002). To gain a better understanding of the Internet skills of the general population, it is important to include people from beyond the academic community (Hargittai, 2002).

Regarding gender, the outcomes are not consistent. Goulding and Spacey (2002) claim that men possess more knowledge about the Internet and the way to use it than women since the latter have been slower to start using the Internet than men have. Wasserman and Richmond-Abbott (2005) found that the level of Internet use was related to Web knowledge, and that this was higher among men than among women. Schumacher and Morahan-Martin (2001) concluded that men possess greater Internet skills than women do. Furthermore, men tend to use the Internet more often than women and for longer periods of time (Kraut et al., 1998; Ono \& Zavodny, 2003; Wasserman \& Richmond-Abbott, 2005). However, recent studies show little variation by gender in access to the Internet in the developed Western countries (Ono \& Zavodny, 2003). Bimber (2000) controlled for socio-economic and demographic characteristics when studying differences in 
gender, and although women were less likely than men to use the Internet frequently, he concluded that this was the product of socioeconomic and other factors, not gender itself. Hargittai and Shafer (2006) found that men and women do not differ greatly in their online abilities, but that women's self-assessed skill is significantly lower than that of men.

When considering age, older people are often regarded as "laggards" in the diffusion process for innovations (Rogers, 1995). Young people get to know the Internet at an early age and thus are considered more skillful than seniors (De Haan, Huysmans, \& Steyeart, 2002). It is often believed that the so-called digital generation possesses the highest level of Internet skills. Seniors have never had the opportunity to acquaint themselves with the Internet at school and lag behind in their ownership and use of computers and the Internet as well as their digital skills (De Haan \& Huysmans, 2002a). Difficulties in learning new skills and resistance to change have also been suggested as barriers to Internet use by the elderly (Castells, 2001). Furthermore, seniors have more trouble learning new digital skills due to decreased working memory and reaction times (Boyd \& Bee, 2009). Those over forty have also missed this opportunity at school, but many of them at least have learned to use computers and the Internet at work. In line with these statements, Hargittai $(2001,2002)$ concludes that age is negatively associated with one's level of Internet skills. Cho, Gil de Zúñiga, Rojas, and Shah (2003) have found that both young and upper-class users were more effective in obtaining what they sought online. Others took indirect or multiple routes to achieve the same end. However, there is also proof that specific Internet skills are insufficient among students (Davis, 2003; Harrison et al., 2003; Klein, Yarnall, \& Glaubke, 2001; Metzger, Flanagin, \& Zwarun, 2003; O’Hanlon, 2002; Volman, Van Eck, Heemskerk, \& Kuiper, 2005).

Education is the most consistent global predictor of the use of ICTs. The higher educated more often own computers, have Internet access at home, and connect through broadband and spend more time online (Buente \& Robbin, 2008; DiMaggio et al., 2004; Robinson, DiMaggio, \& Hargittai, 2003). Strongly related to educational attainment are cognitive resources that are largely responsible for differences in Internet use and in the digital skills of different educational groups (De Haan, Huysmans, \& Steyeart, 2002). Goldin and Katz (2008) argue that the more highly educated are able to keep up with technological advancements and therefore increase their lead over people who are not able to keep up. However, 
they also concluded that education in digital skills cannot keep up with technological developments, which has resulted in wage inequality in the US.

People who spend more time online-whether at work or any other locationwill acquire more knowledge about the Internet and thus develop better online skills (Hargittai, 2002, 2005). Moreover, people who have been Internet users for a longer period of time are expected to be better at finding information online because they have more experience to draw upon (Hargittai, 2002, 2005). Indeed, Hargittai (2002) found years of experience and intensity of use to serve as strong predictors of Internet skills. In general, for both computers and the Internet, the length of previous experience and the amount of current usage have been associated with greater technological expertise (Schumacher \& Morahan-Martin, 2001; Weil \& Rosen, 1995). Young people learn to use a computer at an early age and are considered more skillful. A psychological study of Internet use concluded that most people take at least two years to become competent at finding information online (Eastin \& LaRose, 2000).

Social resources consist of access people have to other sources of help and training (Robinson et al., 2003). Shaw's theory of social constructionism states that individual development is enhanced by constructive activity in the social setting (Shaw, 1995). The user is supported by developing his or her knowledge within a community setting, which helps to reinterpret and develop experiences (Pinkett, 2000). Additionally, learning within the community can, in turn, support the community itself. The individual adds to the knowledge held by the community and to the common setting (Pinkett, 2000). In diffusion of innovations literature, the importance of social support networks in the spread of new technologies is often mentioned. Furthermore, Kraut et al. (1998) presented a "rich get richer" model, which predicts that those who have social support will get more social benefits from using the Internet. The social context of computer and Internet users appears to be a decisive factor in the opportunities they have for learning Internet skills (Van Dijk, 2006). DiMaggio and Hargittai (2001) identified social support as a critical component of inequality. Uptake and further usage of the Internet may be significantly affected by the amount of social support to which a user has access. New users need to be able to draw on social contacts to increase their skill levels and help gain confidence, and they need to be able to have access to emotional support and encouragement when they encounter problems. 
Other factors that might be significant in the possession of Internet skills are socio-economic position, the location of Internet use, and participation in an Internet course. Students and workers are more likely to use the Internet than people that are retired or unemployed (although, as stated previously, undergraduate students with a relatively high level of education also demonstrated limitations in Internet skills). Regarding income, disparities in computer ownership and Internet access are narrowing. However, differences can be observed in the way they are used. People with a higher household income are more likely to seek news and product information, make online travel arrangements, and perform work-related activities online than those with lower income (Madden, 2003). However, they are less likely to use instant messaging or download music (Madden, 2003). People forced to use the Internet at school or in libraries have less time to practice, and that might result in a restriction of their Internet skills. Finally, a myriad of Internet courses are offered both in education and in labor organizations. Those that take part in these courses and learn to use the Internet are more likely to possess sufficient Internet skills than those who do not (Anandarajan, Simmers, \& Igbaria, 2000). Rothbaum, Marthland, and Jannsen (2008) argued that users who lack formal instruction are likely to have trouble using the Internet.

Finally, the existence of a racial digital divide in Internet use has been documented more extensively in the US than in Europe. Although recent data regarding Internet access and types of Internet use is available (e.g., Jackson et al., 2008), to our knowledge, there is no academic research concerning Internet skills and their division over different races among populations at large.

\subsection{Conclusions}

The overview in this chapter reveals several grounds to increase the attention to Internet skills in the social sciences:

- Within different conceptualizations of the digital divide, Internet skills are considered an important issue. In the explanation of different uses of the Internet, the level of Internet skills seems to be one of the most important factors.

- It is being progressively recognized that Internet skills are not equally distributed in society. Differences in Internet skills have the power to 
increase existing social inequalities, since Internet use can have benefits in political, economic, social, health, and cultural dimensions. People with better Internet skills will profit more from Internet use than people who lack these skills.

- Existing figures and research only serve as a poor estimation of Internet skills levels among populations at large. Current research has many problems with reliability and validity, which causes the actual levels of Internet skills to be largely unknown. There is a need for more in-depth and valid research.

The specific Research Question addressed in this chapter is as follows: How do Internet skills relate to inequality in contemporary society? The outline in this chapter revealed that access to the Internet is not simply mapped onto existing inequalities, but has the power to increase existing social divisions in society. From digital divide research, we have learned that besides physical access (although this is a condition), Internet skills are increasingly important for consideration. The meaning of the Internet in people's lives is fully depended on the possession of Internet skills, which are mentioned in all conceptualizations of the digital divide. In fact, having these skills is critical to participate in contemporary information society. When the skills to use the Internet are unequally divided among segments of the population, the consequences of this skills inequality may exacerbate existing societal inequalities. Thus, differences in Internet skills should be considered a new cause for social inequality. Several segments in society are in danger of being unable to meet the increasing communication technology-related demands set upon them. Although inequalities within society have always existed, an even stronger division, in which the higher status members increasingly gain access to more information than the lower status members, seems to exist. 


\subsection{Introduction}

The Internet and its accompanying blitz of technological transformation pushes up against a media model we have long accepted. It forces a shift in paradigms that will make you reevaluate older ways of information gathering even as you learn to incorporate them into the new. The Internet is not a gradual shift in the way we work. Instead, it is an analog-to-digital transformation that will alter the rules of communication.

(Gilster, 1997, p. 38)

The term "literacy" has had a variety of meanings over time. The simplest form of literacy involves the ability to use language in its written form: "A literate person is able to read, write, and understand his or her native language and express a simple thought in writing" (Bawden, 2001, p. 220). A more general definition considers literacy as the skills that are needed to perform well within society. When the context is also considered, being literate becomes "having mastery over the process by means of which culturally significant information is coded" (De Castell \& Luke, 1988, p. 159). In the first chapter, it was argued that within contemporary information society, original forms of literacy should be accompanied by new forms accounting for the massive amounts of information and the supporting technologies. Gilster (1997) suggests that the changes in literacy concepts are based on shifts in communication technology. He focuses on the Internet-based system of communication, information transfer, and information holding and popularized the concept of "digital literacy" as a general ability to understand and use information on the Internet and other digital sources.

Digital literacy is just one of many concepts that resulted from the rapid diffusion of digital technologies into society. Both Bawden (2001) and Virkus (2003) concluded that in most cases, the exact nature of these concepts is not adequately defined. Often, authors seem to believe that the definition of a term used is selfexplanatory. The main goal of this chapter is the provision of a definition that can be used in actual measurements. Since the concern of Internet skills is growing in policy discussions and Internet skills have the power to affect levels of social inequality, there is a strong need for a definition that can guide research. Until now, the development of assessments has been hampered particularly by the lack of consensus on what constitutes measurable dimensions (Ba, Tally, \& Tsikalas, 2002). This chapter provides an answer to Research Question 2 (How can Internet 
skills be defined conceptually?) and 3 (What are the similarities and differences between traditional media skills and digital media skills?).

\subsection{The term "Internet Skills"}

One reason to apply the term "Internet skills" is the association with references applied in digital divide research where the term "digital skills" is commonly used (e.g., De Haan, 2004; Eshet-Alkalai, 2004; Fuchs \& Horak, 2008; Kvasny, 2006; Mason \& Hacker, 2003; Steyeart, 2002; Van Dijk \& Hacker, 2003; Van Dijk, 2005). Internet skills can be considered a specification; others are, for example, the skills to use mobile telephones or computers. Thus, the Internet-adjective of the concept "Internet skills" refers to the medium on which all attention is concentrated in this dissertation. The concept "skills," however, requires further clarification. Several related terms are used interchangeably (e.g., literacy, competence, ability, capacity, expertise, fluency, or know-how). Most common are "literacy," "competence," and "skill," or combinations like "literacy skills" or "literacy competence." Literacy seems to be the most general concept, often considered a set of skills or competencies. Since the goal is to identify measurable dimensions, the terms "skills" and "competences" seem more appropriate than the more general "literacy." The concept of competence has different meanings, and it is not always clear whether competence refers to identifiable indices or is related to patterns of behavior (Anttiroiko, Lintilä, \& Savolainen, 2001; Virkus, 2003). Anttiroiko et al. (2001) concluded that there are two dimensions in competence: knowledge and skills. They found knowledge to be the understanding of how our everyday world is constituted and works, while skills involve the ability to pragmatically apply, consciously or even unconsciously, our knowledge in practical settings. They conceived skills as the technical aspects of competence, or the more technical "how to do" component. This confined technical annotation of skills appears to be the convention, but should be carefully evaluated. The New Oxford Dictionary of English describes skill as "a person's ability to do something well; an expertise." This ability is not limited to technical aspects. In fact, the term "skills" seems to suggest a more (inter)active performance in media use than, for example, the term literacy suggests for reading and writing texts. For instance, using the Internet does not only mean reading and writing on keyboards and screens but also interaction with programs and with other people, transactions in goods and services, and making decisions. This requires more 
action than the passive use of visual media like television or books that mainly require knowledge and cognitive skills. Thus, on top of the more tool-related skills, specific skills are also needed to use the provided information.

\subsection{Overview of related concepts}

Bawden (2001) identified several concepts that are used synonymously for the new forms of literacy, of which the following are relevant for this dissertation: Computer literacy (synonyms: IT, information technology, and electronic literacy), information literacy, network literacy (synonyms: Internet and hypermedia literacy), digital literacy (synonym: digital information literacy), and media literacy. The definitions range from the tautological (e.g., computer literacy is "the ability to use" computers and the Internet) to the idealistic (e.g., "the term literacy is shorthand for cultural ideals as eclectic as economic development, personal fulfillment, and individual moral fortitude" (Tyner, 1998, p. 17)). Bawden (2001) distinguished between skills-based literacies (such as computer literacy)—which essentially indicate skills in handling information in a particular setting, context, or formatand more general literacies.

\subsubsection{Media literacy}

Media literacy as a field of research has developed from the critical evaluation of mass media; originally, it was meant to be a protection from what many people perceived to be the treacherous effects of mass media (Martin, 2006). The term "media literacy" accounts for the more traditional media including print and audio-visual media like radio and television, but also for the Internet. A fairly common and broad concept of media literacy is defined by Potter (2004):

Media literacy is the set of perspectives from which we expose ourselves to the media and interpret the meaning of the messages we encounter. We build our perspectives from knowledge structures. The knowledge structures form the platforms on which we stand to view the multifaceted phenomenon of the media: their business, their content, and their effects on individuals and institutions. The more people use these knowledge structures in mindful exposures, the more they will be able to use media exposures to meet their own goals and the 
more they will be able to avoid high risks for negative effects. (p. 58-59)

This perspective is a bit broader than those of Aufderheide (1993), Silverblatt (1995), Messaris (1998), and Meyrowitz (1998), which purely define media literacy in terms of knowledge of the media. Potter (2004) and others-such as Hobbs (1996), Brown (1998), and Adams and Hamm (2001) —add the notion of skill. Potter lists a number of skills related to media production and seven primary cognitive skills required to attain knowledge:

The skills associated with production (writing, photography, acting, directing, editing, sound recording, etc.) can help people become more media-literate by adding more information to their knowledge structures. But production skills are secondary to the more primary skills of analysis, evaluation, grouping, induction, deduction, synthesis, and abstracting. (p. 59)

Potter and many others working with the traditional concept of media literacy have developed a cognitive view of literacy. However, it is doubtful whether even this enlarged concept of media literacy, encompassing media knowledge and the cognitive (intellectual and analytical) skills required to process media messages, is broad enough to capture the skills required to successfully use the Internet. This requires not only knowledge and cognitive skills, but also practical skills related to (trans)actions, interactions, and all kinds of applications in the context of work, education, and leisure time.

Livingstone, Van Couvering, and Thumim (2005) integrated digital and information technologies into an overall model for media literacy for adults. This model includes multiple media literacy categories that are described medium by medium. In the first of eight categories, named "media access and ownership," the Internet component includes the topic of the digital divide. According to Livingstone et al. (2005, p. 14), this topic should be addressed as a continuum with degrees of marginality and multiple different types of divide, given that the "goalposts of what constitutes acceptable access are continually shifting as new technologies emerge." The second category, "navigating basic media competences," refers to the ability to use the Internet's essential features, such as a browser. 


\subsubsection{Information literacy}

While most definitions of media literacy focus on the nature of various genres of media and the way in which messages are constructed and interpreted, information literacy tends to focus on the ways in which it is accessed and evaluated (Martin, 2006). Information literacy emphasizes the identification, location, evaluation, and use of media materials (Livingstone et al., 2005). In the first chapter, it was pointed out that much confusion exists around the term and that many other terms have been suggested (e.g., infoliteracy, informacy, information empowerment, information competence, information mediacy). Despite the continuous concern about the term, information literacy is the most commonly used phrase (Bawden, 2001). The term first appeared in print in a report by Zurkowski, written in 1974 on behalf of the National Commission on Libraries and Information Science. Zurkowski (1974, p. 6) used the phrase to describe the techniques and skills known by the information literate "for utilizing the wide range of information tools as well as primary sources in molding information solutions to their problems." Information literacy not only stems from technological developments, but also from the emerging information society. Information literacy emerged as a distinct skill set and a necessary key to one's social and economic well-being in an increasingly complex information society (Kulthau, 1987). The American Library Association (1989) used the following concept of information literacy:

The ability to recognize when information is needed and the ability to locate, evaluate, and use the needed information effectively. Ultimately, information literate people are those who have learned how to learn. They know how to learn because they know how knowledge is organized, how to find information and how to use information in such a way that others can learn from them. They are people prepared for lifelong learning, because they can always find the information needed for any task or decision at hand.

Doyle (1994) expands on this definition more specifically and defines an information literate person as someone who:

1. Recognizes that accurate and complete information is the basis for intelligent decision making;

2. Recognizes the need for information;

3. Formulates questions based on information need; 
4. Identifies potential sources of information;

5. Develops successful search strategies;

6. Accesses sources of information including computer-based and other technologies;

7. Evaluates information;

8. Organizes information for practical application;

9. Integrates new information into an existing body of knowledge;

10. Uses information in critical thinking and problem solving.

Shapiro and Hughes (1996) composed a summary of the types of information literacy required in working with computers and networks. They suggested seven components:

1. Tool literacy (the ability to understand and use the practical and conceptual tools of current information technology relevant to education and the areas of work and professional life that the individual expects to inhabit);

2. Resource literacy (the ability to understand the form, format, location, and access methods of information resources, especially daily expanding networked information resources);

3. Social-structural literacy (understanding how information is socially situated and produced);

4. Research literacy (the ability to understand and use the IT-based tools relevant to the work of today's researcher and scholar);

5. Publishing literacy (the ability to format and publish research and ideas electronically, in textual and multimedia forms);

6. Emerging technology literacy (the ability to continuously adapt to, understand, evaluate, and make use of the continually emerging innovations in information technology so as not to be a prisoner of prior tools and resources and to make intelligent decisions about the adoption of new ones); and

7. Critical literacy (the ability to evaluate critically the intellectual, human, and social strengths and weaknesses, potentials and limits, benefits and costs of information technologies).

Similarly, Dunn (2002) describes an assessment that includes a set of seven core competencies for students. In order to be able to find, evaluate, use, 
communicate, and appreciate information in all its various formats, students must be able to demonstrate the following skills:

1. Formulate and state a research question, problem, or issue not only within the conceptual framework of a discipline, but also in a manner in which others can readily understand and cooperatively engage in the search.

2. Determine the information requirements for a research question, problem, or issue in order to formulate a search strategy that will use a variety of resources.

3. Locate and retrieve relevant information, in all its various formats, using, when appropriate, technological tools.

4. Organize information in a manner that permits analysis, evaluation, synthesis, and understanding.

5. Create and communicate information effectively using various media.

6. Understand the ethical, legal, and sociopolitical issues surrounding information.

7. Understand the techniques, points of view, and practices employed in the presentation of information from all sources.

There are strong similarities between the various statements of information literacy skills developed to date (Dunn, 2002). Regardless of how one describes the essential skills and knowledge necessary for information literacy, the processes themselves are always the same (Dunn, 2002). Virkus (2003) argued that the increasing attention to information literacy is partly the result of information overload, especially related to the growth of digital information. Although Bundy (2004) notes that the most important elements of information literacy are critical discernment and reasoning rather than technological fluency, information literacy definitions typically seem to involve both computer-specific knowledge as well as broader content-related skills like evaluating information sources. In general, definitions of information literacy can be divided into three concepts (Boekhorst, 2003):

- The ICT concept: Information literacy refers to the competence to use ICT to retrieve and disseminate information.

- The information (re)sources concept: Information literacy refers to the skills to find and use information independently or with the aid of intermediaries. 
- The information process concept: Information literacy refers to the process of recognizing information need, retrieving, evaluating, using, and disseminating information to acquire or extend knowledge.

\subsubsection{Computer, IT, and ICT Iiteracy}

In 1981, soon after the popularization of the personal computer, a concept that elaborated the special skills required for the use of computers was invented and published in the Washington Post (Warschauer, 2003). The term used was "computer literacy," a very narrow concept only indicating basic forms of computer operation like turning on a computer, opening a folder, and saving a file. Unfortunately, such narrow definitions of skills required for computer use have remained customary since that time. In educational settings, computer literacy often results in tool-oriented approaches, which causes the teaching to be reduced to relatively trivial software instruction (Hoem \& Schwebs, 2004). The lack of in-depth definitions of computer literacy might stem from the fact that there is a pressing need to promote computer literacy, because computer literacy is a requisite for finding and using networked information (Breivik, 2001).

Like computer literacy, IT and ICT literacy are often described narrowly and only cover the basic skills to use a computer and networks. Basic skills include the skills necessary to handle specific tools (e.g., the ability to use a search engine, a word processor, or the configuration of input and output devices) (Adams, 1984). While such definitions are specific and measurable, they quickly become obsolete because of the rapid changes in technology ( $\mathrm{Ba}$ et al., 2002). In addition, these tool-dependent definitions enumerate countless specific skills, begging the question of whether literacy might entail the development of more general capabilities that people may apply across tools and settings (Ba et al., 2002). More in-depth definitions of ICT literacy connect the term with other traditional and new human capabilities, such as literacy and numeracy; scientific, economic, technological, visual, and information-handling abilities; thinking and productivity capacities; and communication abilities (Armstrong \& Warlick, 2004; Markausakite, 2006). In 2001, the Educational Testing Service (ETS) convened an international ICT literacy panel to study the growing importance of existing and emerging ICTs and their relationship to literacy, called ICT Literacy. The panel emphasizes that "technology skills alone, without corresponding cognitive skills and general literacy, will not decrease the gaps defined by a digital divide." Their 
working definition of ICT literacy is as follows: "ICT literacy is using digital technology, communication tools, and/or networks to access, manage, integrate, evaluate, and create information in order to function in a knowledge society" (ETS, 2001, p. 2). The panel emphasizes that ICT literacy cannot be defined primarily as the mastery of technical skills and concludes that the concept should be broadened to include both critical cognitive skills as well as the application of technical skills and knowledge. The cognitive skills include general literacy and critical thinking and problem solving.

\subsubsection{Digital literacy}

Gilster (1997, p. 1) defined digital literacy as "the ability to understand and use information in multiple formats from a wide range of sources when it is presented via computers" and "...being able to understand a problem and develop a set of questions that will solve that information need." He suggests that the most essential aspect is "the ability to make informed judgments about what you find on-line," paralleling definitions of information literacy. Gilster emphasizes that the Internet is an inclusive medium, which means that no central organization determines the content of the Internet and that there is no sense of accountability to a mass audience. He also emphasizes that the Internet places demands that were always present, though less visible, in the analog media of newspaper and television. Gilster put forward the idea that new media require new skills for both navigating networked technologies and interpreting the meaning of digital messages. Gilster did not provide specific skills, but argued that acquiring digital literacy for the Internet involves mastering a set of core competencies (as cited in Bawden, 2001):

- The ability to make informed judgments about what is found online, "the art of critical thinking;"

- The skills of reading and understanding in a dynamic and non-sequential hypertext environment;

- Knowledge assembly skills; building a "reliable information horde" from diverse sources, with "the ability to collect and evaluate both fact and opinion, ideally without bias;"

- Searching skills, essentially based in Internet search engines;

- The ability to manage the "multimedia flow," using information filters and agents; 
- The ability to create a "personal information strategy," with selection of sources and delivery mechanisms;

- Awareness of other people and the ability to contact them to discuss issues and get help;

- Being able to understand a problem and develop a set of questions that will solve that information need;

- Understanding of backing up traditional forms of content with networked tools;

- Wariness in judging validity and completeness of material referenced by hypertext links.

Eshet-Alkalai (2004) considers digital literacy to involve more than the mere ability to use software or operate a digital device; it includes a large variety of complex cognitive, motor, sociological, and emotional skills, which users need in order to function effectively in digital environments. Her concept of digital literacy includes:

- Photo visual literacy: "Reading" instructions from graphical displays;

- Reproduction literacy: Utilizing digital reproduction to create new, meaningful materials from pre-existing ones;

- Information literacy: Evaluating the quality and validity of information;

- Branching literacy: Constructing knowledge from non-linear, hyper-textual navigation;

- Socio-emotional literacy: Understanding the "rules" that prevail in cyberspace and applying this understanding in online cyberspace communication.

Eshet-Alkalai and Amichai-Hamburger (2004) presented empirical data to support the concept. They conducted a study in which subjects had to complete assignments. The subjects were selected over three age categories: young (11th graders averaging age 17), middle (third-year college students averaging age 26), and older (college graduates, averaging age 37). The results revealed that regarding photo-visual literacy, there were no significant differences between young and medium age groups, but both were significantly different from the oldest group, which performed worse. Regarding reproduction literacy (editing a neutral text story about a child getting ready for school), the oldest age group performed the best, followed by the middle and the youngest. Branching literacy was measured by letting the subject plan an online trip. Results indicate that there were no significant differences between the two younger groups, who both scored 
significantly higher than the oldest. Information literacy was measured by analyzing (looking for bias and contradiction) seven Internet news sources and presenting an overall written summary of opinion on quality and reliability for each source. The oldest group performed best, followed by the middle and then the young. Socio-emotional literacy subjects had to enter a chat room and engage in a dialogue on a political topic. The middle group scored highest, followed by the youngest, followed by the oldest on a combined variable that included cognitive presence, social presence, and emotional presence.

\subsubsection{Network literacy}

McClure (1994, p. 138) introduced the term "network literacy" and defined it as "the ability to identify, access, and use electronic information from the network." McClure (as cited in Bawden, 2001) suggests that the basic components of network literacy include:

- Knowledge:

- An awareness of the range and uses of networked resources;

- An understanding of the role and uses of networked information in problem solving and "basic life activities;"

- An understanding of the system by which networked information is generated, managed, and made available.

- Skills:

- An ability to retrieve specific types of information from networks;

- An ability to manipulate networked information; combining, enhancing, adding value;

- An ability to use networked information to help make work-related and personal decisions.

\subsubsection{Multidisciplinary definitions}

New forms of literacy are considered to help researchers achieve a better understanding of Internet use in the context of digital inequality (DiMaggio et al., 2004). However, from the outline above, it seems that there are several approaches possible, all with their own strengths and weaknesses. To propose a conceptual definition appropriate for the general Internet user population, the most suitable aspects of each should be combined. It appears to be important to 
avoid a technologic viewpoint when defining Internet skills. Internet skills should contain both technical aspects that are related to the use of the medium and substantive aspects that are related to the content provided by the medium. Definitions of information literacy already account for this distinction, but tend to be aimed at students instead of the general user population. More appropriate is the definition used by Warschauer (2003) who made a list containing the concepts of computer literacy, information literacy, multimedia literacy, and computermediated communication literacy. He defined computer literacy in terms of the basic forms of computer and network operation, information literacy as the ability to manage vast amounts of information, and multimedia literacy as the ability to understand and produce multimedia content. He added computer-mediated communication literacy as the skill of effectively managing online communications (e.g., email, chatting, and video-conferencing) to adhere to the rules of "netiquette." Mossberger, Tolbert, and Stansbury (2003) considered technical competence (the more narrow set of skills to operate the Internet) and information literacy (the skills to recognize when information can solve a problem or fill a need and to effectively employ information resources).

Both Steyeart (2002) and Van Dijk (2005) introduced three general types of skills applicable to both computers and the Internet. Steyeart distinguished between the following three types of skills:

- Instrumental skills: the operational manipulation of technology;

- Structural skills: relating to the structure in which information is contained;

- Strategic skills: the basic readiness to pre-actively look for information, information-based decision-making, and scanning of the environment for relevant information.

According to Steyeart, Internet skills are mostly about new instrumental skills, while underneath, the traditional information and strategic skills are generic to all media. In the transformation towards an information society, they significantly gain importance. Van Dijk (2005) considered information skills as characteristic of the information society. He further developed the concept of digital skills as a sequence of operational skills, information skills, and strategic skills needed to work with computer media. He changed Steyeart's definition to the following:

- Operational skills: the skills to operate computer and network hardware and software; 
- Information skills: the skills to search, select, and process information in computer and network sources. In a further specification of information skills, he proposes the following division:

- Formal information skills: the ability to understand and to handle the formal characteristics of a computer and a computer network-the Internet—such as file structures, menu structures, and hyperlinks;

- Substantial information skills: the ability to find, select, process, and evaluate information in specific sources of computers and networks according to specific questions and needs.

- Strategic skills: the capacities to use these sources as the means for specific goals and for the general goal of improving one's position in society.

\subsection{Proposing an Internet skills definition}

Both Steyeart (2002) and Van Dijk (2005) suggest that their definitions are applicable to the modalities of both computer and Internet use. Their definitions include functional skill that should be considered a requirement of typical Internet use, common to the majority of Internet users. Despite the sequential and conditional nature of their concepts, they claim the skills can and should be measured separately. Both definitions account for the technicalities of Internet use, apparent in the instrumental or operational and formal information skills, and for the aspects that relate to the content provided by the Internet and are included in the substantial information and strategic skills. These skills largely correspond with the definitions of information and digital literacy. The distinction between medium and content is important and will serve as the main division in the proposed definition for Internet skills. Medium-related skills correspond with some definitions of computer literacy, operational skills as defined by Van Dijk (2005), and instrumental skills as defined by Steyeart (2002). Furthermore, the formal information skills proposed by Van Dijk should be considered independent of the provided content (as will be explained in Section 3.4.2) and should be added to the medium-related part of Internet skills instead of the information or content-related part. The content-related part of Internet skills should follow the definitions of information literacy, digital literacy, substantial information skills, and strategic Internet skills. These all relate to the content provided by the medium, in this case, the Internet. For providing measurable dimensions of the concept of Internet skills, four skills are considered. On the one 
hand, operational Internet skills and formal Internet skills are both related to the use of the Internet as a medium. On the other hand, information Internet skills and strategic Internet skills are both related to the content provided by the Internet. This division provides opportunities to investigate how these different Internet skill levels are distributed among segments in the population. Furthermore, these skill divisions can be applied to the more traditional media.

\subsubsection{Operational Internet skills}

Besides the concepts of operational skills, instrumental skills, computer literacy, and ICT literacy, other terms used to indicate a set of basic skills in using computer or Internet technology are technical competence (Mossberger et al., 2003), technological literacy (Carvin, 2000), and technical proficiency (Søby, 2003). According to Carvin, technological literacy is the ability to utilize common IT tools, including hardware, software, and Internet tools like search engines. Søby describes technical proficiency as the basic component of digital literacy, including a foundational knowledge of hardware, software, applications, networks, and elements of digital technology. Both Steyeart and Van Dijk refer to the "European computer driving license" for measuring operational and instrumental skills. Here, the Internet skill aspects consist of the following:

- Understanding concepts and terms associated with using the Internet;

- Appreciating security considerations;

- Accomplishing common Web search tasks using a Web browsing application and available search engine tools;

- Bookmarking Web sites and printing Web pages and search outputs;

- Navigating within and completing Web-based forms;

- Understanding concepts associated with electronic mail.

The University of Valencia (2000) uses four items for testing Internet skills:

- Create a bookmark or save a favorite Web page;

- Save/download a Web site with graphics, video, or audio;

- Follow a link from one Web page to another Web page;

- Move forward and backward through Web pages.

Bunz (2004) developed an instrument to assess people's fluency with the computer, e-mail, and the Internet to fill the existing void between previously 
developed computer literacy or experience scales and the ever-accelerating development of Internet technology. To measure Web fluency, Bunz used items related to using a Web browser:

- Opening Web addresses;

- Identifying host servers from Web addresses;

- Using "back" and "forward" buttons to move between Web pages;

- Using search engines.

Larsson (2002) created a Digital Literacy Checklist after Gilster's (1997) definition of the concept "digital literacy." Items related to the Internet include the following:

- I know what a Web browser is and how to use it to move between Web sites or pages;

- I know how to use my browser's bookmark file to organize Web sites;

- I know how to change the preferences on my browser. (e.g., I know how to change my opening page and the font type and size);

- I've emptied the disk and memory cache on my browser to free up space on my hard drive;

- I can read various file formats on the Web (e.g., GIF, JPG, PDF, Word);

- I can use Telnet (an Internet program) to log on to another computer and use that computer as if it were my own;

- I'm aware that I can download (and/or listen to) audio files;

- I understand that HTML is used to tag ASCII text for display by Web Browsers;

- Once I have located data on the Web, I can identify the format and know how to move it into my desktop for further analysis;

- I can download files using my Web browser;

- I can reliably transfer text and binary files between client and server computers (i.e., move data between my desktop and mainframe computers) using FTP;

- I can download files from public FTP sites;

- I know how to set up a personal newsfeed so that I only get the information I need.

Based on Larsson's checklist, Revere (2005) created a Digital Literacy SelfAssessment. The Internet skill part includes the following questions:

- Have you ever used a browser?

- Have you ever saved a Web page or printed a Web page? 
- Have you ever customized a Web browser (security settings, tool bars, etc.)?

- Do you know the difference between a search engine, subject directory, and meta-search tool and know when it is most advantageous to use one?

- If you found a site on the Web that you particularly liked, would you know how to easily bookmark that site so you could go back to it later without having to re-enter the entire address?

- Do you know how to view, download, decompress, and open documents and programs from Internet sites (e.g., graphics, PDF files)?

- Once you have located data on the Web, do you know how to move it onto the desktop or save it to a folder?

- Have you emptied the disk and memory cache on your browser to free up space on the hard drive?

Table 3.1 summarizes the discussed skills. Furthermore, indicators used by Statistics Netherlands (CBS) are added. Not all aspects in Table 3.1 are considered in the definition that will be proposed. Navigational aspects are discussed in the next section, wherein the formal Internet skills are introduced. Setting browser preferences is not a prerequisite for using the Internet, and neither are security considerations, printing, or creating Web pages.

When measuring operational Internet skills, the following aspects should be considered:

- Operational use of an Internet browser (typing URL in the address bar);

- Operational use of a search engine (input field and search operation);

- Using (back and forward) browser buttons;

- Bookmarking Web sites;

- Downloading or saving files from the Internet;

- Saving Web pages and Web sites;

- Operational use of online forms (different buttons and sending forms);

- Using various file formats (e.g., pdf).

Operational Internet skills are the basic skills needed to use the Internet and for which it is easiest to predict skill level because most of the skills research-based on surveys-focuses on them. It is also relatively easy to observe them in performance tests. Research shows that individuals in Western countries also show relevant differences with regard to these skills (e.g., De Haan, 2003; Hargittai, 2002; Park, 2002). Young users often have the operational skills that 
support Internet access (Clark, 2001). According to De Haan and Huysmans (2006), operational skills are mastered relatively poorly by the elderly, unemployed, and disabled, as well as by homemakers. Many senior citizens reportedly lack basic knowledge of Internet operation (Lundt \& Vanderpan, 2000). The performance tests conducted by Hargittai (2002) revealed that the general American user population lacks an understanding of the basics of surfing the Internet.

Table 3.1 Measurements of Operational Internet Skills

\begin{tabular}{|c|c|c|c|c|c|c|}
\hline $\begin{array}{l}\text { ECDL } \\
(2009)\end{array}$ & $\begin{array}{l}\text { Valencia } \\
(2000)\end{array}$ & $\begin{array}{l}\text { Bunz } \\
(2004)\end{array}$ & $\begin{array}{l}\text { Larsson } \\
(2002)\end{array}$ & $\begin{array}{l}\text { Revere } \\
(2005)\end{array}$ & $\begin{array}{l}\text { CBS } \\
(2009)\end{array}$ & \\
\hline $\begin{array}{l}\text { Understand } \\
\text { concepts }\end{array}$ & & $\begin{array}{l}\text { Use a } \\
\text { browser }\end{array}$ & $\begin{array}{l}\text { Know what a } \\
\text { browser is }\end{array}$ & $\begin{array}{l}\text { Use a } \\
\text { browser }\end{array}$ & & Browser use \\
\hline & Follow links & & & & $\begin{array}{l}\text { Surf the } \\
\text { Internet }\end{array}$ & Navigation \\
\hline $\begin{array}{l}\text { Use a search } \\
\text { engine }\end{array}$ & & $\begin{array}{l}\text { Use search } \\
\text { engines }\end{array}$ & & $\begin{array}{l}\text { Know } \\
\text { differences } \\
\text { between } \\
\text { search tools }\end{array}$ & $\begin{array}{l}\text { Use search } \\
\text { engines }\end{array}$ & $\begin{array}{l}\text { Search engine } \\
\text { use }\end{array}$ \\
\hline Bookmark & Bookmark & & Bookmark & Bookmark & Bookmark & Adding \\
\hline Web site & Web site & & Web site & Web site & Web site & bookmarks \\
\hline & $\begin{array}{l}\text { Move forward } \\
\text { and back }\end{array}$ & $\begin{array}{l}\text { Use back } \\
\text { and forward }\end{array}$ & $\begin{array}{l}\text { Move } \\
\text { between } \\
\text { Web sites }\end{array}$ & & & Navigation \\
\hline & & & $\begin{array}{l}\text { Download } \\
\text { audio files } \\
\text { Move files to } \\
\text { desktop }\end{array}$ & $\begin{array}{l}\text { Move files } \\
\text { onto the } \\
\text { desktop or } \\
\text { folder }\end{array}$ & $\begin{array}{l}\text { Download } \\
\text { files from the } \\
\text { Internet }\end{array}$ & $\begin{array}{l}\text { Downloading } \\
\text { files }\end{array}$ \\
\hline & $\begin{array}{l}\text { Save a Web } \\
\text { site }\end{array}$ & & & $\begin{array}{l}\text { Save a Web } \\
\text { page }\end{array}$ & & Saving files \\
\hline $\begin{array}{l}\text { Print Web } \\
\text { pages }\end{array}$ & & & & $\begin{array}{l}\text { Print Web } \\
\text { pages }\end{array}$ & & Printing \\
\hline \multirow[t]{6}{*}{$\begin{array}{l}\text { Navigate / } \\
\text { complete } \\
\text { forms }\end{array}$} & & & & & & Using forms \\
\hline & & & $\begin{array}{l}\text { Change } \\
\text { browser } \\
\text { preferences }\end{array}$ & $\begin{array}{l}\text { Customize } \\
\text { browser }\end{array}$ & & $\begin{array}{l}\text { Browser } \\
\text { preferences }\end{array}$ \\
\hline & & & $\begin{array}{l}\text { Empty disk } \\
\text { and cache }\end{array}$ & $\begin{array}{l}\text { Empty disk } \\
\text { and cache }\end{array}$ & & \\
\hline & & & $\begin{array}{l}\text { Read various } \\
\text { file formats } \\
\text { on the Web }\end{array}$ & & & $\begin{array}{l}\text { Use different } \\
\text { file formats }\end{array}$ \\
\hline & & & & $\begin{array}{l}\text { View, } \\
\text { decompress, } \\
\text { and open } \\
\text { documents }\end{array}$ & & \\
\hline & & $\begin{array}{l}\text { Identify host } \\
\text { server }\end{array}$ & & & & \\
\hline $\begin{array}{l}\text { Consider } \\
\text { security }\end{array}$ & & & & & & $\begin{array}{l}\text { Security } \\
\text { considerations }\end{array}$ \\
\hline & & & & & $\begin{array}{l}\text { Create } \\
\text { Web site }\end{array}$ & $\begin{array}{l}\text { Create Web } \\
\text { page }\end{array}$ \\
\hline
\end{tabular}




\subsubsection{Formal Internet skills}

The second type of Internet skills is formal Internet skills². Every medium has particular formal characteristics that have to be understood and requires techniques that must be mastered. Formal skills relate to the structures on which a medium is built. The Internet is the most obvious example of hypermedia, requiring the skills of browsing and navigating. Users must have these skills to use the vast and diverse number of Web sites and menu layouts offered on the Web. Web sites differ in (the placement of) text, content, backgrounds, photos, frames, links, buttons, frames, and pop-ups. Furthermore, they differ in features used to help the user navigate the pages; elements such as color for text, links, and hyperlinks visited; multimedia elements such as sound, animation or video; and interactive features such as message boards and forms. Similar features account for the design of menus (e.g., static or rollover). All of these distinct characteristics demand navigational skills on the part of the user. Many sites use features without sufficient attention to human factors, which renders these sites inaccessible to all but users with sophisticated know-how about Web navigation (DiMaggio \& Hargittai, 2001). Often, Web sites seem to be perfectly usable from the perspective of the developers but perform different in actual usability tests. In this regard, studies usually assume a technical perspective in which the design of the Web site is tested. Of course, this helps to improve Web site functionality, but it neglects users' skills deficiencies.

The most frequently cited problem in hypermedia use is disorientation (Ahuja \& Webster, 2001; Lee, 2005). Most traditional media are linear, giving the user little control over the flow of information. Hypermedia provides a formal structure that enables users to choose their own non-linear paths instead of employing the fixed formal structures of print media (for example, chapters, paragraphs, and references). Now, users can move not only forward but also backward and to unknown locations (Kwan, 2001). Without a sense of location, distance, and necessary direction, it is not surprising that users often have a strong sense of disorientation (Conklin, 1987; Kwan, 2001). Compared to the computer, the Internet is a much larger and more complex problem domain. Not knowing where one is, where to go next, how to get back to a previous site, what path one has followed, or where to look for information is all part of the sense of losing orientation (Edwards \& Hardman, 1989; Park \& Kim, 2000). Disorientation arises

\footnotetext{
${ }^{2}$ Inspired by Van Deursen \& Gui (2008).
} 
from the need to know where one is in the network, where one came from, and how to get to another place in the network (Balasubramanian, 1994). Disorientation can result in frustration, loss of interest, and a measurable decline in user efficiency (McDonald \& Stevenson, 1998). When following a pre-defined path rather than browsing freely, users are less likely to feel disoriented or lost (Zellweger, 1989). However, the structure of the Internet changes dynamically: pages disappear, reappear in alternative form, or are mirrored on other servers; new pages appear, and so on (Fuchs, 2008). It seems that the complexity of the Internet increases with its growth. Getting lost on the Internet also occurs when users have sufficient domain expertise. This means that disorientation should be framed in terms of site structure, Web links and Web design, independent of the information topics being navigated (Danielson, 2003; Webster \& Ahuja, 2006). Formal Internet skills are considered part of medium-related skills, like operational Internet skills. Formal Internet skills then consist of the skills necessary to use different Web site layouts and maintain one's orientation when browsing.

Navigation and orientation are important skills within Web site menus, within Web sites, when moving between Web sites, and when browsing through search results. Problems in doing this are often ascribed to seniors ${ }^{3}$. Indeed, the Internet presents obstacles to users with mobility or cognitive impairments, and navigation can be difficult (O'Hara, 2004). Clicking within small areas or selecting options from menus requires fine motor control and good sight. Furthermore, navigating a loosely organized nonlinear site can frustrate people with short-term memory impairments (Nielsen, 2002). Becoming lost or disoriented is known to be one of the most significant problems in hypertext navigation, but there have been only a few attempts to assess and quantify lostness. Lostness in hypertext is difficult to measure (Herder, 2003; Otter \& Johnson, 2000). Ahuja and Webster (2001) conducted an experiment demonstrating that user-perceived disorientation in Web navigation (assessed using a questionnaire developed by the authors) is only weakly related to user behavior and that perceived disorientation is a better predictor of performance (time) than user behavior (such as the number of visited Web pages and page revisits). Using questionnaire-based measures of lostness is difficult if not impossible (Gwizdka \& Spence, 2007). There is some evidence that

\footnotetext{
${ }^{3}$ In the media, seniors and their problems with new media like the Internet are often stereotyped. They create the perception that elderly adults are technophobes and are fearful or mistrustful of new technology. Unfortunately, this also affects the elderly themselves, further strengthening the hesitation to use the Internet.
} 
hypertext causes confusion when compared to linear structures (Miall \& Dobson, 2001). Eshet-Alkalai and Amichai-Hamburger (2004) have explored the level of "branching literacy" by testing the ability to plan a trip on the Internet. However, this task requires the consideration of the whole range of skills as defined in this proposal. A more appropriate task would, for example, be to extract very simple information (e.g., contact information) from Web sites that are very different in their layout and menu designs.

\subsubsection{Information Internet skills}

Before the diffusion of the Internet in households, searching within electronic databases was usually facilitated by individuals skilled in the use of databases, such as librarians (Jenkins, Corritore \& Wiedenbeck, 2003). Because of the ubiquity of the Internet and search engines, most users have never had formal training in the use of searching facilities (Tatcher, 2008). Strongly related to information Internet skills are the definitions of information literacy and digital literacy. Standards and statements regarding these terms are produced by several professional information associations, all derived from the widely accepted definition of the American Library Association (Correia \& Teixeira, 2003). Literature relevant to information Internet skills is spread across different areas that tend to remain separate. This causes the aspects of information retrieval science to be poorly integrated (Jenkins et al., 2003). Most literature focuses on the search for (online) information, which is mostly seen as an action via which users try to fulfill their information needs. In the related literature, there exists general agreement that the process entails more than merely gathering information: it encompasses posing or identifying a question or problem, exploring available information, refining the question, gathering and evaluating information, and synthesizing and using information (Wallace, Kupperman, Krajcik, \& Soloway, 2000). If the goal is to define information Internet skills, studies that adopt a staged approach in explaining this process are the most interesting.

Ellis (1989) and Marchionini (1995) propose a generally accepted distinction that was originally developed in the context of traditional information retrieval. While the distinction proposed by Ellis is based on the searching behavior of social scientists, research physicists and chemists, and engineers and research scientists in industrial firms (Choo, Detlor, \& Turnbull, 2000), the distinction proposed by 
Marchionini is recursive and appropriate for digital environments like the Internet. Furthermore, it is not limited solely to the information-seeking process in search engines. Many scholars of online searching have adapted aspects from this framework. Based on Marchionini's model, it becomes obvious that different users have different needs at different points in a search process:

1. The process starts with problem definition. The identification of the information need can be regarded as the determination or definition of a problem (Brand-Gruwel, Wopereis, \& Vermetten, 2005). A problem definition is comprehensive when a clear description of the problem and the type and amount of information required to solve it are provided. The phase of problem definition is addressed in a literature review conducted by Walraven, Brand-Gruwel, and Boshuizen (2008). They stated that people above the age of 18 do not seem to experience many problems relating to this skill. However, this lack of problems could also be explained by the lack of focus on skills underlying defining the information problem (Timmers \& Glas, 2010).

2. Once the information problem has been formulated, sources of information to use in solving the problem are considered. Interesting sources of information are based on criteria such as reliability, validity, precision, completeness, accuracy, availability, novelty, and costs (BrandGruwel et al., 2005). Step 2 of the Big6 (Eisenberg \& Berkowitz, 1990), a well-known information problem-solving model used in education, includes determining possible information sources. People have many options for searching on the Internet (such as search engines, meta-search tools, bots, directories, jump pages, and specialized resources like those that search the Invisible Web). However, most people searching for information turn to the same system for all their tasks (Jansen \& Pooch, 2000).

3. The third step, formulating search queries, has gained considerable attention in academic research, highlighting a potential barrier to information for different types of Internet users. The typical Internet user only uses one or two terms per query (Aula \& Nordhausen, 2006). A recurring mistake is the use of multiple terms without any spaces between them (Birru et al., 2004; Hargittai, 2002). In addition, students experience 
similar problems (Aula \& Nordhausen, 2006; Mittermeyer, 2005). They do not come up with other search queries as presented in an informationsearch assignment and almost never try alternate search queries when the initial search attempt fails (Bilal, 2002; De Vries, Van Der Meij, \& Lazonder, 2008; Dinet, Favart, \& Passerault, 2004). Besides search queries, other criteria can be used to refine search results (e.g., Boolean operators, date, location, format, and other criteria). Users searching for information mostly use simple operations, with an average of two terms per query and two queries per session (Jansen \& Pooch, 2000). Furthermore, only about two percent of the queries contain query operators (Beitzel, Jensen, Chowdhury, Grossman, \& Frieder, 2004). In a study among students, Ivanitskaya, O'Boyle, and Casey (2006) found that only few used advanced search options or limited their searches. Many seemed to rely on basic searches (while understanding that a one-word search would return too many documents).

4. For the fourth step, selecting the most relevant information or search results, people must be able to separate relevant from irrelevant documents. When a search engine only returns a few results, they can be inspected comprehensively. However, when people use broad searching strategies in tandem with large-scale search engines, a vast number of often-unsuitable results will appear (Livingstone, Van Couvering, \& Thumim, 2005). This problem is reinforced by the fact that information seekers often do not venture past the first page of search results (Aula \& Nordhausen, 2006; Birru et al., 2004; Ozmutlu, Spink, \& Ozmutlu, 2004). Jansen and Spink (2003) report that Web search engine users on average view about eight Web documents, with more than $66 \%$ of searchers examining fewer than five documents in a given session. Furthermore, on average, users view about two to three documents per query, and over $55 \%$ of Web users view only one result per query (Jansen \& Spink, 2003).

5. The final step is the ability to make informed judgments about what is found online, "the art of critical thinking" (Gilster, 1997). Information is not always of the same quality, and its diversity requires specific information skills from users (Steyeart, 2002). It is necessary to check for data accuracy and verify the reliability of the sources. In particular, the ability to discriminate between authoritative information and information 
whose provenance is detached from its originator is very important (Steyeart, 2000). This is key in evaluating most of the new media and creates a need for citizens to acquire new capabilities related to assessing the value, veracity, and reliability of information if they are to participate effectively in a global society (Mansell, 2002). Eshet-Alkalai (2004) equates the evaluation aspect to the concept of information literacy, referring to the cognitive skills required for the educated and effective evaluation of information. Regarding children, it is proven that most lack the skills to critically evaluate the information found online (Fidel et al., 1999; Lorenzen, 2001; Pritchard \& Cartwright, 2004; Shenton \& Dixon, 2003).

\subsubsection{Strategic Internet skills}

Strategic skills relate to the usage gap, as described by Bonfadelli (2002) and Van Dijk (2005), between those who primarily use information and communication technologies and networks for professional and educational development and those who mainly use them for entertainment purposes. Van Dijk defines strategic skills as the capacity to use computer and network sources as the means of reaching particular goals and for the general goal of improving one's position in society. Pruulmann-Vengerfeldt (2006) relates strategic skills with Bourdieu's discussion of how economic, social, and cultural capital influences everyday life and activities. These forms of capital increase the value and usability of the Internet and are stronger in people with better strategic Internet skills. "If there were no privileged consequences as a result of Internet usage, there would be little to debate other than percentage point difference in access and usage over time for various groups" (Mason \& Hacker, 2003, p. 41). Although strategic skills hardly depend on operational and formal skills alone, together with information skills, they serve as the means to reach a particular goal by one's own initiative.

Although Doyle (1994) mentions problem solving in his definition of information literacy, and the American Library Association (1989) includes decision-making, very few skill-related studies available address strategic Internet skills. Here, a process that consists of four analytically distinct steps in making effective use of the Internet is proposed. This process is based on the classical approach to decision-making whose emphasis lies on procedures through which decision- 
makers can reach an optimal solution as efficiently as possible (Miller, 2006). The first step is goal orientation. In this context, this means being aware of the opportunities offered by the Internet and subsequently determining the goal of the Internet session. Keeping an eye on this goal and working toward it is difficult, especially in a digital media landscape that offers an enormous number of distracting stimuli. The second step is taking the right actions on the Internet. In correspondence with the decision-making process, here, this step means gathering and combining various online information sources to achieve the best means of reaching the desired goal (Miller, 2006). After the right actions are taken, it is time to make decisions about how to reach the original goal by using the (oftenexcessive amount of) information retrieved selectively; this is the third step. In the decision-making process, this step means developing a set of decision options and evaluating them according to carefully developed criteria (Miller, 2006). The decision-making process ends when an optimal decision is identified and decision implementation can begin (Miller, 2006). The final step is that of obtaining the benefits from making the optimal decision. These benefits can be of an economic, political, social, health, or cultural nature. Considering all four steps, we can reflect on the following subsequent indicators of strategic Internet skills: developing an orientation toward a particular goal, taking the right actions to reach this goal, making the right decisions to reach this goal, and eventually gaining the benefits resulting from this goal.

Citizens with a high degree of strategic skills should be able to make the most out of their use of the Internet (e.g., putting together a trip, gaining financial benefits, choosing between political parties, or finding a hospital that specializes in treating a specific disease). Unfortunately, to our knowledge, there is no research that addresses these skills on an individual level. Regarding orientation, research reveals that older adults are particularly susceptible to distracting material while reading (Connelly, Hasher, \& Zacks, 1991). They therefore experience greater difficulty than younger adults do on the Internet. Regarding the step of taking action, users tend to become loyal to a single Web site for accomplishing tasks, even when a better Web site is just a click away (Zauberman, 2003). Especially for strategic questions, it might be very important to compare information from different resources to make sure the potential benefits are optimal (e.g., in comparing trips offered on different Web sites). 


\subsubsection{Proposing an Internet skills definition}

The four Internet skills categories are based on individual abilities and are a requirement of typical Internet use. They are relevant for the general population to function well in an increasingly digital environment. An important aspect is that the four Internet skills can be divided into both medium (operational and formal) and content (information and strategic) related Internet skills. People often believe that using the Internet only requires medium-related skills that are automatically learned by younger generations. An explanation for this somewhat shortsighted view might be that research into traditional media skills has often focused on the analysis of messages provided by the media (Livingstone, 2003). Medium-related operational and formal Internet skills are less often discussed in traditional media skill research because these skills were and are more or less taken for granted. With the arrival of computers and the Internet, new skills that appeared to be difficult to master were suddenly required, demanding all attention. The contentrelated aspects of the Internet were thus neglected. Answering Research Question 2, Table 3.2 on the next page contains the definition of Internet skills.

The proposed definition avoids a technologically deterministic viewpoint by both accounting for technical aspects related to the use of the Internet and substantive aspects related to the content provided by the Internet. The provided concept of Internet skills is of a sequential and conditional nature. Content-related skills somehow depend on the medium-related skills because the absence of mediumrelated skills means that one will not even come to perform the content-related skills.

\subsection{Traditional media skills and new media skills}

The distinction between medium (operational and formal) and content (information and strategic) related skills is applicable to both traditional and new media. The common denominator of operational skills is that the use of all media requires certain technical abilities to perceive and to process the signs and symbols concerned and to command the particular hardware and software. All media have particular formal structures that users should become familiar with too. Subsequently, all media offer particular contents that enable searching, selecting, processing, and evaluating information. Finally, all media can be approached with 
Table 3.2 Four Types of Internet Skills

\section{Operational Internet skills}

- $\quad$ Operating an Internet browser:

- Opening Web sites by entering the URL in the browser's location bar;

- Navigating forward and backward between pages using the browser buttons;

- Saving files on the hard disk;

- Opening various common file formats (e.g., PDFs);

- Bookmarking Web sites.

- Operating Internet-based search engines:

○ Entering keywords in the proper field;

- Executing the search operation;

- Opening search results in the search result lists.

- Operating Internet-based forms:

○ Using the different types of fields and buttons;

- Submitting a form.

\section{Formal Internet Skills}

- Navigating on the Internet by doing the following:

- Using hyperlinks embedded in different formats such as texts, images, or menu's.

- Maintaining a sense of location while navigating on the Internet, meaning:

- Not becoming disoriented when navigating within a Web site;

- Not becoming disoriented when navigating between Web sites;

- Not becoming disoriented when opening and browsing through search results.

\section{Information Internet Skills}

- Locating required information, by:

- Choosing a Web site or a search system to seek information;

- Defining search options or queries;

- Selecting information (on Web sites or in search results);

- Evaluating information sources.

\section{Strategic Internet skills}

- Taking advantage of the Internet by doing the following:

- Developing an orientation toward a particular goal;

- Taking the right action to reach this goal;

- Making the right decision to reach this goal;

- Gaining the benefits resulting from this goal.

Content-related

Internet skills 
strategic skills to use them as a means to reach a particular benefit or goal. Investigating how the proposed Internet skill definition is also applicable to other media provides a better understanding of what, exactly, is new about the skills required to use the Internet. To gain further understanding, traditional media skills (print and audio-visual media) and new media skills (computer and Internet) are discussed and compared. In Table 3.3, the four skills are ordered sequentially. This table shows that audio-visual media users should be able to read and write. Computer users need some understanding of audio-visuals. Internet users have to be able to operate a computer. Without the skills of reading and writing and without understanding graphics or audio-visuals, they can barely use this medium either.

\subsubsection{Traditional media skills}

The minimum skills required to derive meaning from print media are the abilities to read and write, labeled as operational skills in Table 3.3. Explaining this figure from a column view, it can be observed that in print media, to start with, the operational skills of reading and writing have received the most attention by educators and policy makers. On the other hand, the formal skills to make use of print media are often neglected. They are the skills to understand and possibly edit the structures of texts. Formal skills are necessary to understand the way texts are organized. These are the chapters, paragraphs, tables of content, indexes, and the references used in books, magazines, journals, newspapers, etcetera. Together, operational and formal skills form the necessary basis to use any (print) medium.

As with the Internet, for print media, the information and strategic skills relate to the provided content. Information skills for using a print medium are the skills to search for information, select relevant information, process the information, and evaluate the information found. Applying information skills in print media can be hard, since these media are mainly text-based and require a lot of mental effort to extract narrative meaning (Newhagen \& Bucy, 2004; Salomon, 1979). Strategic skills relate to the purpose for using the print medium and the potential empowerment that this usage accompanies. From the information obtained, a user might take actions, make decisions by placing this information in the right perspective, and finally gain certain benefits that affect his/her personal or professional life. 


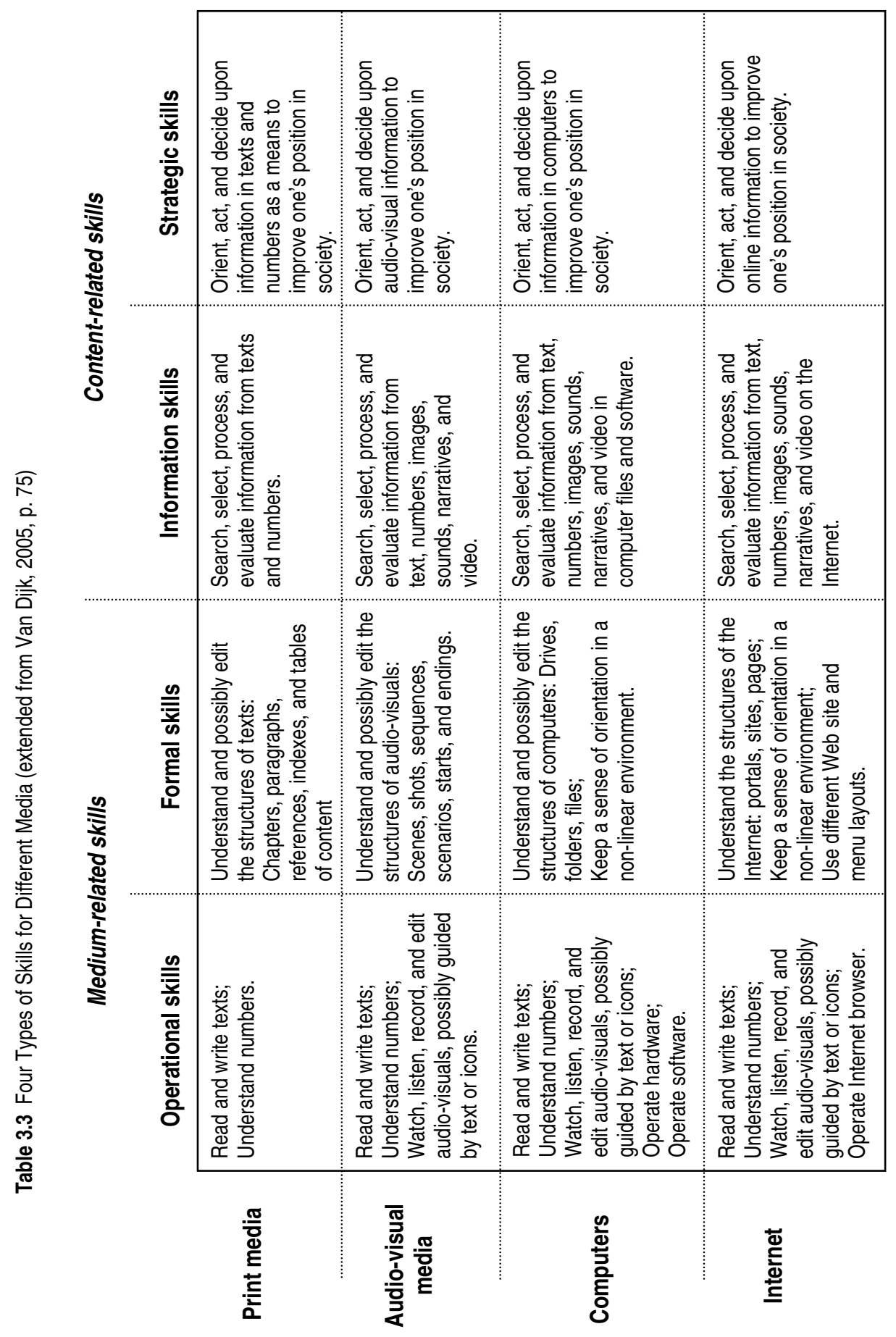


Traditional media literacy also encompasses audio-visual media like television programs, movie pictures, and videos. Originally, audio-visual media mainly provided sounds, video, or images that could largely be processed without print media skills (Reeves \& Nass, 1996). After some time, audio-visual media became increasingly filled with text in introductions, subtitles, textboxes, and banners. Besides reading and writing, operational skills for using audio-visual media encompass attentive and systematic watching, listening, and recording audiovisuals. Formal skills needed for audio-visual media include the understanding and possibly editing of the structures of audio-visuals: scenes, shots, sequences, scenarios, starts, and endings. Information and strategic skills that belong to the content provided by the audio-visual media require different mental operations than processing texts (for example, processing images and sounds). However, the set of actions required—searching, selecting, processing, and evaluating information — can be described using the same concepts. Regarding strategic skills, it is relevant to know which types of audio-visual information can be used for taking actions and for making decisions. When the goal is information retrieval, the actions required will be different from those required to meet the goal of entertainment (Salomon, 1979).

Knowledge can be derived from both print and audio-visual media. The characteristics of traditional media (e.g., low levels of selectivity and accuracy of information as compared to digital media) stimulate a relatively passive manner of using these media. While traditional media enable active mental processing, digital media require a minimum level of active engagement with the medium, and they offer the possibility for interaction and transaction.

\subsubsection{Digital media skills}

People often think that using digital media such as computers and the Internet only requires button-knowledge and that this will be automatically learned by the younger generation. An explanation might be that traditional media literacy research has often focused on the analysis of messages provided by the media (Livingstone, 2003). With the arrival of the new media, suddenly new skills that appeared to be difficult to master were required. The new operational and formal skills have attracted most of the attention. The operational skills necessary to use computers are partly similar to those required for the use of print and audio-visual 
media. The ability to read and write text and figures and to watch, listen to, record, and edit audio-visual materials are skills that can also be used while operating computers. This list of skills is then complemented by special skills necessary for the operation of computer hardware and software. The formal skills one employs in using computers are also new since they entail a structure different from that of any traditional medium: one must know how to use drives, folders, files, and so on. This structure needs to be understood and mastered before effective use is possible. Not only should one know how to open folders and save files, but one should also be able to maintain a sense of orientation while browsing through the numerous files and folders that hard drives often contain. Both operational and formal skills are necessary for the use of digital media. However, a high level of operational and formal skills is not in itself sufficient. The content-related skills for using computers-information and strategic skills-are similar to the equivalent skills in traditional media. In using computers, information skills include searching, selecting, processing, and evaluating information from integrated digital texts, images, sounds, videos, and figures. Strategic skills include becoming orientated, acting, and making decisions in processing this information in order to reach a particular goal and eventually gain a personal or professional benefit.

Operational, formal, information, and strategic skills to use the Internet have already been extensively discussed. An important difference between the Internet and other media is that the Internet requires a unique form of digital skills for adequate use of a browser and hypermedia. Furthermore, although the information skills required for traditional media and computers are partly similar to those needed on the Internet, information provided by the Internet is infinitive and thus puts much more pressure on the content-related skills. Online searches often result in the appearance of a large amount of unsuitable results, making the selection part of information skills harder to achieve. Subsequently, sources found should also be evaluated for their validity and reliability. Strategic Internet skills assume additional analytic abilities. Not only should information sources be analyzed, but distinctions should also be made between goals and means and between what is more and less important to reach these goals. 


\subsubsection{Differences between traditional and digital media skills}

In answering Research Question 3 (What are the similarities and differences between traditional media skills and digital media skills?), it appears that traditional and digital media skills contain similarities but also some important differences:

- The characteristics of traditional media (e.g., low potential of selectivity and accuracy of information) create relative passivity in those using these media. In this respect, traditional media usage is different from predominant digital media use. While traditional media enable active mental processing, digital media require users to interact with interfaces. A minimum level of active engagement with the medium is required, and the possibility of interactions, transactions, and interpersonal communication is offered. Using computers or the Internet constitutes action, interaction, and transaction.

- Since the storage capacity, accuracy, and selectivity of digital media are greater than those of traditional media (Van Dijk, 2006), the contentrelated skills (information skills and strategic skills) gain more emphasis. As discussed in the first chapter, no other media provides such a large amount of information and range of non-linear choices. The Internet's information features are used in ways similar to those in which print and audio-visual media are employed. The acts of searching, selecting, processing, and evaluating information from online video, images, sounds, texts, and numbers largely correspond to the information skills used with traditional media and computers. However, the information provided by the Internet is infinite and linked in a non-linear manner, which puts much more stress on the content-related skills. For example, when a broad search strategy is used in an Internet search engine, a large number of unsuitable results will appear, making the selection element of information skills harder. The sources found should also be evaluated for their validity and reliability.

- Strategic Internet skills assume additional analytic abilities. Not only should information sources be analyzed, but distinctions should also be made between goals and means and between what is more and less important in reaching these goals. In order to acquire strategic skills and employ them on the Internet, users must be critical and analytical, and they must have superior information skills. 
On the one hand, computers and the Internet make things easier, because they enable systematic, simultaneous information retrieval from innumerable sources. Finding information in a traditional library might be more difficult for inexperienced information seekers than finding the same information on the Internet using a straightforward search engine. However, as stipulated by the observed differences, on the other hand, computers and the Internet make information seeking and benefiting from media use more difficult, because this assumes a number of new operational and formal skills to begin with. This raises an extra barrier in addition to that presented by the skills of reading and writing. Additionally, digital media require particular information and strategic skills that are partly different from those required for the use of traditional media.

The differences imply that digital media skills increase the inequality observed in the possession of traditional media skills. Livingstone (2003) argues that the social uses of information technologies work to reproduce and reinforce traditional literacy skills rather than to transform them or to generate new literacy skills. Steyeart (2002) and Van Dijk (2005) emphasize that information and strategic skills pose new demands and will be much more important in future information and network societies. An increasing number of activities are affected by purposeful searches for information, as well as by the processing and use of the information to attain or retain positions in all kinds of relationships, both in work and social life. 


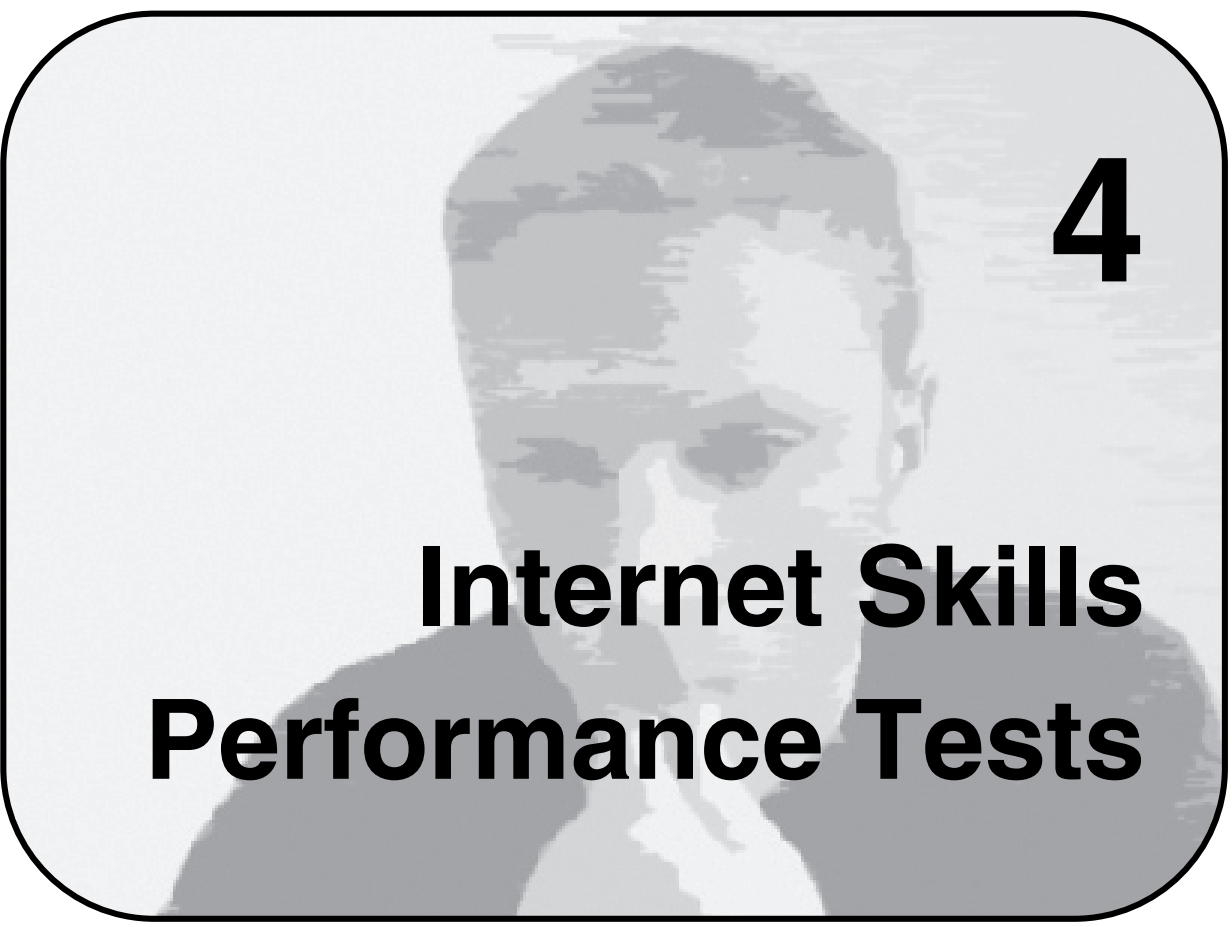

To measure the level of Internet skills among the Dutch population, three studies are conducted in which subjects are asked to complete assignments on the Internet. The three studies differ in the applied context-government, general-leisure, and health information services, respectively. After introducing the studies, methods for measuring Internet skills are presented, followed by an overview of the eventual method applied here. The chapter continues with a presentation of the absolute levels of Internet skills and the most important contributors to Internet skills levels. After evaluating the proposed Internet skills definition, conclusions are presented. 


\subsection{Introduction}

The technological opportunities the Internet is offering right now are just a tiny reflection of what can be reality in the beginning of the next millennium.

Quotes such as the one above (taken from the 1994 Dutch e-government development program "Electronic Highway") reveal that the expectations of the Internet as a means for public service delivery were very high in the nineties. As a result, almost all public authorities developed online information and services to serve their citizens, thereby following upon other (commercial) domains. The policy to offer services and information online seems to be characterized by barely funded presuppositions of the citizens' skills when using the Internet. An insufficient level of Internet skills makes the information and services unavailable when they are offered as an alternative to their traditional counterparts. In this chapter, the fourth (What is the level of Internet skills among the Dutch population?) and fifth (Which factors determine Internet skills levels?) research questions are addressed.

To indicate the level of Internet skills of the Dutch population, three studies are conducted in which subjects have to complete assignments on the Internet. The observation method applied is discussed in Section 4.2. The assignments in the first study (discussed in Section 4.3) relate to information and services offered online by public authorities. The results reveal how ready Dutch citizens are for the public transformations towards the Internet in which a substantial amount of time and money is invested. The assignments in the second study (discussed in Section 4.4) are more general (leisure-related), since it might be argued that people with higher levels of bureaucratic knowledge might perform better on the content-related skills (information and strategic Internet skills) in the first study than people who lack such knowledge. The assignments in the third study (discussed in Section 4.5) apply the domain of health information. While about $60 \%$ of Americans believe that online health information is similar or even better than what they get from a physician (Diaz et al., 2002), it appears that in the domain of health, only about $30 \%$ of the information provided online is reliable (Eysenbach \& Köhler, 2002). The quality of the information offered varies by language as well as disease (Eysenbach, Powell, Kuss, \& Eunryoung, 2002) and is likely to be determined by the supplier, which can be a commercial (perhaps not identified as such) or a non-profit organization. The increasing importance of the Internet as a source of health information and the apprehensions of health 
professionals about the quality of online health information raise serious concerns (Morahan-Martin, 2004).

In the final section of this chapter, the data collected in the three performance tests are used to evaluate the Internet skills definition. The performance tests allow for an evaluation of the definition's usefulness, reliability, and validity. In Section 4.8, Research Question 6 (What is the reliability and validity of the proposed definition of Internet skills adopted as a particular type of scientific measurement?) is addressed.

\subsection{Method}

\subsubsection{Choosing a measurement method}

For studying the levels of Internet skills among populations at large, a variety of methodologies can be employed. Most of the existing research used surveys that might offer in-depth explorations of participants' skills, but in most cases gathered data based on people's own perceptions or estimations of their computer or Internet skills (Kuhlemeier \& Hemker, 2007). People are presented with a list of skills and are asked to evaluate how well they perform those skills. Although selfreport questionnaires have advantages — such as the ability to present a large number of questions on a wide range of skills in a short time, simple scoring, fast processing, and cost effectiveness (Kuhlemeier \& Hemker, 2007) - this method has significant problems of validity (Hakkarainen et al., 2000; Hargittai, 2005; McCourt, Larres, Ballantine, \& Whittington, 2003; Merritt, Smith, \& Renzo, 2005; Talja, 2005). Interpretations of skills are perspective and context dependent (Talja, 2005). Talja introduced the concept "Information Technology-self" (IT-self), which is a dialogic, context-dependent, momentary, and multilayered entity involving different temporal layers with which people refer to their past experiences but also predict future levels of skills. Individuals produce different kinds of IT-selves in different contexts (e.g., creation of an online learning environment versus creating a homepage), and their IT-self-skills depend on detailed changes in dialogic contexts of discussion and argumentation (Talja, 2005). Talja also denotes that individuals' descriptions of their own Internet skills depend upon with whom they compare themselves (Talja, 2005). Merritt, Smith, and Renzo (2005) checked the validity of self-reports concerning computer skills 
and found that these were rated higher than actual skills. Although, in general, higher self-efficacy leads to a greater likelihood of using the Internet (Eastin \& LaRose, 2000) and to a higher completion of online tasks (Torkzadeh \& Van Dyke, 2002), this kind of measurement has significant problems of validity and is a poor predictor of performance. Experience shows that males, especially young males, overrate their performance, while some women and elderly people underrate it (Hargittai \& Shafer, 2006). Research among young students revealed that girls appear to have a more realistic view of their own digital skills than boys (De Haan \& Huysmans, 2002b; Hakkarainen et al., 2000). Consequently, it is not clear to what extent differences in self-ratings correspond to real differences in skills. Besides measures of self-efficacy, surveys also employ indirect measures for Internet skills (see the examples provided in the first chapter). They do not measure actual skills and therefore can only be considered as a poor indication. In fact, they measure differences in Internet applications used, not the skills needed for these applications.

The measurement of Internet skills should provide the possibility to actually use the Internet. Observational studies could be very suitable to provide a realistic view of people's Internet skills. However, their cost is a strong limitation for largescale data gathering. Although observational studies discourage interruption of participants-which makes it difficult to obtain contextual information (Kellar, Hawkey, Inkpen, \& Watters, 2008)—and testing the actual skills of Internet users in a behavioral study is a highly labor-intensive process, they seem to be the most suitable methods of obtaining a direct measure of skill. A variety of process indicators may be automatically recorded, such as user actions and successful completion. To obtain a realistic view of people's Internet skills, three observational studies are conducted in which subjects are asked to complete assignments on the Internet. The procedures followed are described below and to some extent correspond with experimental settings (controlled environment, presence of a test-leader, applied quota samples). Due to the labor-intensive process, it is not possible to obtain a representative sample of the whole Dutch population. However, obtaining a reliable and valid measurement is the primary objective. 


\subsubsection{Sample}

In line with the procedure used by Hargittai (2002), we used the condition of invitation that the subject used the Internet at least once every month for more than just e-mail. This condition excluded approximately 20\% of the Dutch population, but ensured that low-frequency users who were nonetheless familiar with the Internet were included. The subjects were not informed about the exact intention of the studies. They were told the study would cover general Internet use. The invitation policy put people who feared a test at ease. Only adult Dutchspeaking users were included in the three studies.

To be able to generalize from the findings, the subjects were recruited by applying a stratified random sampling method. First, a sample was randomly selected from a telephone book of the region of Twente (an eastern region in the Netherlands). Subsequently, a selective quota sample was drawn from the strata of gender, age (equal number of subjects in the categories of age 18-29, 30-39, 40-54, and 55-80), and educational level of attainment (equal number of subjects in the categories low, middle, and high) to reach equal subsamples. Digital divide research has repeatedly shown that access to and use of the Internet is heavily stratified by these variables. When respondents indicated they were willing to participate, their contact mailing address and e-mail address were recorded, and a time for the research session was scheduled. Respondents received a confirmation/follow-up letter in the mail and directions to the research site. The day before the study, respondents were reminded of the session by phone. After the session (which took approximately one and a half hours), subjects were rewarded with 25 Euro for their participation.

Both in the first and second study, 109 subjects participated (response rates to the invitation were $28 \%$ and $32 \%$ respectively). In the third study, 88 subjects participated (response rate was 19\%). Table 4.1 contains the characteristics of the subjects. The quota sample applied was designed to ensure a sufficient amount of subjects in different gender, age, and educational groups to obtain valid data. The average years of Internet experience was $8.1(\mathrm{SD}=3.0), 8.3(\mathrm{SD}=3.2)$, and 9.3 $(\mathrm{SD}=4.3)$, and the average amount of Internet use was 9.7 hours a week $(\mathrm{SD}=9.7)$, 9.5 hours a week $(\mathrm{SD}=10.2)$, and 12.3 hours a week $(\mathrm{SD}=13.7)$ in studies 1,2 , and 3 , respectively. The average age of the subjects was 43.4 ( $\mathrm{SD}=15.5), 43.2$ 
$(\mathrm{SD}=17.1)$, and $42.5(\mathrm{SD}=17.5)$, respectively. Overall, the people who participated in the three studies represent a diverse group of Internet users.

Table 4.1 Subjects over Gender, Education, Age, Location of Internet Use, Assistance Requirements, Socio-economic Status, and Participation in an Internet Course

\begin{tabular}{|c|c|c|c|c|c|c|c|}
\hline & \multicolumn{2}{|c|}{ Study 1} & \multicolumn{2}{|c|}{ Study 2} & \multicolumn{2}{|c|}{ Study 3} \\
\hline & & $\mathrm{n}$ & $\%$ & $\mathrm{n}$ & $\%$ & $\mathrm{n}$ & $\%$ \\
\hline \multicolumn{8}{|c|}{ Gender } \\
\hline & Male & 51 & 47 & 57 & 52 & 45 & 51 \\
\hline & Female & 58 & 53 & 52 & 48 & 43 & 49 \\
\hline \multicolumn{8}{|l|}{ Age } \\
\hline & $18-29$ & 25 & 23 & 27 & 25 & 24 & 27 \\
\hline & $30-39$ & 27 & 25 & 24 & 22 & 18 & 21 \\
\hline & $40-54$ & 27 & 25 & 28 & 26 & 23 & 26 \\
\hline & $55-80$ & 30 & 28 & 30 & 28 & 23 & 26 \\
\hline \multicolumn{8}{|c|}{ Education } \\
\hline & Low (e.g., Primary school) & 32 & 29 & 34 & 31 & 25 & 28 \\
\hline & Middle (e.g., High school) & 37 & 34 & 34 & 31 & 32 & 36 \\
\hline & High (e.g., College and University) & 40 & 37 & 41 & 38 & 31 & 35 \\
\hline \multicolumn{8}{|c|}{ Primary location of Internet use } \\
\hline & At home & 95 & 87 & 104 & 95 & 75 & 85 \\
\hline & At work & 13 & 12 & 5 & 5 & 1 & 1 \\
\hline & At school & 1 & 1 & 0 & 0 & 8 & 9 \\
\hline & At a friend's or family member's home & 0 & 0 & 0 & 0 & 3 & 3 \\
\hline & At a library & 0 & 0 & 0 & 0 & 1 & 1 \\
\hline \multicolumn{8}{|c|}{ Assistance required when using the Internet } \\
\hline & No & 51 & 47 & 45 & 41 & 49 & 56 \\
\hline & Yes, from family & 32 & 29 & 29 & 26 & 18 & 21 \\
\hline & Yes, from friends & 18 & 17 & 27 & 25 & 17 & 19 \\
\hline & Yes, from colleagues & 5 & 5 & 5 & 5 & 4 & 5 \\
\hline & Yes, from a helpdesk & 2 & 2 & 3 & 3 & 0 & 0 \\
\hline \multicolumn{8}{|c|}{ Socio-economic status } \\
\hline & Employee & 54 & 50 & 52 & 47 & 30 & 34 \\
\hline & Retired & 18 & 17 & 19 & 18 & 14 & 16 \\
\hline & Student & 14 & 13 & 16 & 15 & 21 & 24 \\
\hline & Homemaker & 10 & 9 & 11 & 10 & 3 & 3 \\
\hline & Employer & 7 & 6 & 5 & 5 & 6 & 7 \\
\hline & Disabled & 5 & 5 & 3 & 3 & 4 & 5 \\
\hline & Unemployed & 1 & 1 & 2 & 2 & 9 & 10 \\
\hline \multicolumn{8}{|c|}{ Participation in an Internet course } \\
\hline & No & 84 & 77 & 81 & 74 & 63 & 72 \\
\hline & Yes & 25 & 23 & 28 & 26 & 25 & 28 \\
\hline
\end{tabular}




\subsubsection{Data collection and procedure}

The first study took place between September and December 2007, the second study between September and December 2008, and the third study between October 2009 and April 2010. All three performance tests were conducted in a University office, which had both shortcomings and advantages. Shortcomings include the negative influence on response rates, the use of a location with which people are not familiar, and the use of a computer that is configured differently from the machine people usually use. This may influence the results, as certain settings (e.g., the default home page or keyboard layout) are not equivalent to their own. Advantages include the fact that this approach controls for quality of the Internet connection and of hardware/software and that the setting is equally different and new for all.

After arriving at the laboratory, subjects were given verbal instructions about the procedure of the study. Prior to the test, a 10-minute questionnaire was administered to gather personal data. After the subjects completed the questionnaire, they were given a sequence of nine assignments, one at a time. Subjects themselves decided when they were finished or wanted to give up on an assignment. No encouragements were given because the pressure to succeed is already higher in a laboratory setting than at home. After a specific maximum amount of time had passed (determined from the pilot-tests), the test-leader gently asked the subjects to move on to the next assignment. If the correct answer was not found, the task was rated as not completed. The test-leader refrained from influencing the subjects' strategies. Subjects were offered snacks and beverages during the sessions.

During the assignment completion, subjects used a keyboard, a mouse, and a 17inch monitor. These were connected to a laptop from which the test-leader could watch the subjects' actions. The laptop had access to a high-speed university network for Internet use and was programmed with the most popular Internet browsers (Microsoft Internet Explorer, Mozilla Firefox, and Google Chrome). This allowed subjects to replicate their regular Internet use. No default page was set on the browsers, and all the assignments started with a blank page. To ensure that subjects were not influenced by previous user's actions, the browser was reset after each session by removing temporary files, cookies, and favorites. In addition, downloaded files, history, form contents, and passwords were removed, and the 
laptop was rebooted. To address Research Questions 7 and 8 (discussed in Chapter 5), Morae Recorder (TechSmith, Version 2.2) was used to record the screen actions of the subjects that completed the assignments (see Chapter 5).

The test-leader directly measured whether the task was completed successfully and the time spent on the tasks. These two measures are the two most important for the level of performance in research concerning search performance (Aula \& Nordhausen, 2005). The task completion rate is considered the most important outcome. However, since a user can be very slow but eventually find the correct answer, whereas another could find the answer almost immediately, the time spent on the assignments was also used as a measure of success. For every skill type, several assignments are prepared consisting of multiple tasks, so the total outcome for every skill is measured as the number of tasks solved successfully and the time spent on these tasks. Both completion and time were directly noted during the sessions.

After finishing the assignments, a second survey was administered to the subjects. This survey included scaled items that together represent the four types of Internet skills. Because the three performance tests are labor-intensive and expensive, another goal is to suggest ways in which Internet skills can be assessed by survey questionnaires instead of always relying on elaborate data collection methods. This is further discussed in Chapter 6 .

\subsubsection{Assignments}

Two assignments (consisting of eight tasks) were used to measure operational Internet skills, two (consisting of four tasks) for measuring formal Internet skills, three for measuring information Internet skills, and two for measuring strategic Internet skills. The total outcome for every type of Internet skills is measured as the number of tasks solved successfully and the time spent on these tasks. The assignments applied situations that are closely linked to types of real-life questions that people face, since the presence of a real information need makes observations a means of offering a valuable insight into users' natural behavior (Shneidermann, Byrd, \& Croft, 1997). All assignments are fact-based and have a specific correct action or answer. Open-ended tasks are avoided because of the ambiguity of interpretation of the many potential answers. Before the three performance tests 
were conducted among the participating subjects, 12 subjects were recruited to participate in a pilot-test in which the assignments were tested for comprehensibility and applicability. A conscious effort was made to include seniors and less-educated individuals. After the pilot-tests, some formulations in the task descriptions were adjusted.

In the first study, the assignments related to government information and services. Some of the assignments were derived from the ten most used search queries in the general Dutch government's Internet portal. For the assignments that directed subjects to a specific Web site, Web sites with a high score on usability in official government benchmark studies were selected. In Appendix I, all assignments in study 1 and the corresponding skills indicators from the Internet skills definition proposed in Chapter 3 are overviewed. The second study used a more popular context, in which general, leisure-related assignments geared toward the consciousness of all Internet users had to be completed. Appendix II contains the assignments used in the second study and the corresponding Internet skill indicators. The assignments in the third study were all health-related and accessible to the general user population. These are overviewed in Appendix III.

\subsubsection{Operationalization of variables}

Several explanatory variables that are discussed in Chapter 2 are accounted for in the Internet skills measurements. Gender is included as a dichotomous variable and age as a continuous variable. Data on education were collected by degree. These were subsequently divided into three overall groups of low, medium, and higher educational attainment. Internet experience was measured as the number of years people have been using the Internet. The amount of Internet use was measured by the number of hours respondents spend browsing the Internet weekly (the week before the survey was used as reference). Furthermore, participation in an Internet class, whether at school or elsewhere, was considered dichotomous (no/yes). In the regression analyses reported later, the data on social support, location of Internet use, and working situation, as reported in Table 4.1, are also transformed to dichotomous variables. Working situation was divided into active (employers, employees, and students) or inactive (retired, disabled, homemakers, unemployed) groups. This transformation was performed because the number of subjects in some of these groups is very low (e.g., the number of disabled or unemployed subjects) and because adding these as dichotomous 
variables provides information about the variables as a whole, instead of each separate group.

\subsubsection{Missing data}

The number of missing values was low. During the assignment completion, the test-leaders were instructed to check all data carefully for missing answers. If these were found, the subjects were asked to provide the answer. This resulted in only two missing values in the first study, three in the second study, and none in the third study. In the regression analyses reported in the next sections, these variables are neglected. However, the other available data of the subjects that forgot to answer a question were still used, given the small number of subjects in the studies.

\subsection{Results of performance test 1: Government information}

\subsubsection{General results}

Altogether, the subjects completed an average of $78 \%$ of the operational Internet skill tasks, $72 \%$ of the formal Internet skill tasks, and $62 \%$ of the information Internet skill tasks. Most problematic are the two strategic Internet skill tasks of which the subjects completed an average of $22 \%$ (see Table 4.2). The time the subjects spent on the assignments varied substantially. Some subjects, for example, needed less than three minutes for the eight operational Internet skills tasks, while others were still not finished within 20 minutes (see Table 4.2).

Table 4.2 Overview of Successful Task Completion and Time Spent

\begin{tabular}{lccrcc}
\hline Independent variables & \multicolumn{2}{c}{ Task completion } & \multicolumn{3}{c}{ Time spent (seconds) } \\
& $\mathrm{M}(\mathrm{SD})$ & $\%$ & $\mathrm{M}(\mathrm{SD})$ & Min. & Max. \\
\hline Operational tasks (8) & $6.26(1.89)$ & 78 & $553(254)$ & 167 & $1200^{*}$ \\
Formal tasks (4) & $2.86(1.04)$ & 72 & $616(255)$ & 242 & $1200^{*}$ \\
Information tasks (3) & $1.86(0.84)$ & 62 & $939(449)$ & 257 & 2157 \\
Strategic tasks (2) & $0.44(0.65)$ & 22 & $1466(575)$ & 437 & 2719 \\
\hline
\end{tabular}

${ }^{*}$ maximum time allowed

Table 4.3 reveals that $39 \%$ of the subjects were able to complete all operational Internet skills tasks, 33\% were able to complete all formal Internet skills tasks, 
$21 \%$ were able to complete all information Internet skills tasks, and only $11 \%$ of the subjects were able to complete both the strategic skills tasks. As much as $62 \%$ of the subjects could not complete either of the two strategic Internet skills tasks successfully. The hardest task, which only $20 \%$ of the subjects were able to complete successfully, was finding out which of three political parties had the subject's first, second, and third favor. Three issues had to be considered (nuclear energy, childcare allowance, and having two nationalities).

Table 4.3 Number of Tasks the Subjects Failed to Complete Successfully

\begin{tabular}{lcc}
\hline & \# of failed tasks & \% of subjects \\
\hline Operational Internet skills & 0 & 39 \\
& 1 & 17 \\
2 & 10 \\
3 & 11 \\
4 & 12 \\
5 & 5 \\
& 6 & 5 \\
& 7 & 1 \\
& 8 & 0 \\
\hline Formal Internet skills & 0 & 33 \\
& 1 & 32 \\
& 2 & 22 \\
Information Internet skills & 3 & 11 \\
& 4 & 1 \\
\hline Strategic Internet skills & 0 & 21 \\
& 1 & 52 \\
& 2 & 18 \\
& 3 & 8 \\
\hline
\end{tabular}

\subsubsection{Internet skills determinants}

According to Table 4.4, age and level of educational attainment appear to be strong contributors to the operational Internet skills level, significant both for the number of tasks completed successfully and the time spent on the tasks. Internet experience is also significant for the number of tasks completed and for the time spent on the operational Internet skill tasks. Hours spent online weekly did not contribute to either the number of operational Internet skills tasks completed or the time spent on the assignments. The latter was determined by working 
situation. Active people (employees, employers, and students) required less time than inactive people (disabled, homemakers, retired, and unemployed).

Table 4.4 Linear Regression on Number of Operational Tasks Completed Successfully and Time Spent

\begin{tabular}{|c|c|c|c|c|}
\hline \multirow[t]{2}{*}{ Independent variables } & \multicolumn{2}{|c|}{ Task completion } & \multicolumn{2}{|c|}{ Time spent } \\
\hline & $t$ & $\beta$ & $t$ & $\beta$ \\
\hline Gender (M/F) & -0.82 & -.06 & -1.30 & -.08 \\
\hline Age & -3.13 & $-.30^{\star \star \star}$ & 5.11 & $.43^{\star \star *}$ \\
\hline Education (low-medium-high) & 3.86 & $.32^{\star \star \star}$ & -2.75 & $-.27^{\star \star \star}$ \\
\hline Years online & 1.90 & $.15^{\star}$ & -2.56 & $-.18^{\star \star}$ \\
\hline Hours online weekly & 0.55 & .04 & -1.44 & -.10 \\
\hline Internet class (no / yes) & 0.45 & .03 & -0.14 & -.01 \\
\hline Assistance required (no / yes) & -1.47 & -.12 & 1.83 & .13 \\
\hline Primary location of Internet use (at home / elsewhere) & 1.15 & .08 & -1.15 & -.07 \\
\hline Working situation (inactive / active) & 1.62 & -.15 & -1.97 & $-.16^{*}$ \\
\hline$R^{2}$ & & .52 & & .64 \\
\hline$F$ & & $14.02^{\star \star \star}$ & & $22.34^{\star * *}$ \\
\hline
\end{tabular}

Regarding formal Internet skills, again, age and educational level of attainment appeared as significant contributors to both the number of successfully completed tasks and the time spent on the formal Internet tasks. Requiring assistance and the primary of location of Internet use also played significant roles regarding the number of completed formal Internet skill tasks. The less people need help, and the more often they use the Internet at home, the better their formal Internet skills (see Table 4.5).

Table 4.5 Linear Regression on Number of Formal Tasks Completed Successfully and Time Spent

\begin{tabular}{|c|c|c|c|c|}
\hline \multirow[t]{2}{*}{ Independent variables } & \multicolumn{2}{|c|}{ Task completion } & \multicolumn{2}{|c|}{ Time spent } \\
\hline & $t$ & $\beta$ & $t$ & $\beta$ \\
\hline Gender (M/F) & 1.06 & .08 & -2.17 & -.15 \\
\hline Age & -2.58 & $-.25^{\star \star}$ & 5.01 & $.46^{\star \star \star}$ \\
\hline Education (low-medium-high) & 2.94 & $.26^{*}$ & -1.98 & $-.16^{\star}$ \\
\hline Years online & 1.56 & .13 & -1.68 & -.13 \\
\hline Hours online weekly & -0.30 & -.02 & -1.66 & -.13 \\
\hline Internet class (no / yes) & 1.00 & .07 & -0.24 & -.02 \\
\hline Assistance required (no / yes) & -3.08 & -.26 & 1.65 & .13 \\
\hline Primary location of Internet use (at home / elsewhere) & -2.40 & $-.18^{\star \star}$ & -0.76 & -.05 \\
\hline Working situation (inactive / active) & 1.26 & $.12^{\star}$ & -1.07 & -.09 \\
\hline$R^{2}$ & & .49 & & .57 \\
\hline$F$ & & $12.39^{\star \star \star}$ & & $16.46^{\star * *}$ \\
\hline
\end{tabular}


The regression results reported in Table 4.6 indicate that educational level of attainment is a significant contributor to the number of information tasks completed successfully. No other variables in the equation appear significant. The same accounts for the time spent on the information Internet skill tasks. Here, also, the educational level of attainment is the only significant contributor.

Table 4.6 Linear Regression on Number of Information Tasks Completed Successfully and Time Spent

\begin{tabular}{lcccc}
\hline Independent variables & \multicolumn{2}{c}{ Task completion } & \multicolumn{2}{c}{ Time spent } \\
& $t$ & $\beta$ & $t$ & $\beta$ \\
\hline Gender (M/F) & -1.35 & -.13 & -2.17 & -.15 \\
Age & -0.89 & -.12 & 5.01 & $.46^{\star * \star}$ \\
Education (low-medium-high) & 3.12 & $.36^{\star * *}$ & -1.98 & $-.16^{*}$ \\
Years online & 0.60 & .07 & -1.68 & -.13 \\
Hours online weekly & -1.02 & -.11 & -1.66 & -.13 \\
Internet class (no / yes) & 0.27 & .02 & -0.24 & -.02 \\
Assistance required (no / yes) & -0.00 & .00 & 1.65 & .13 \\
Primary location of Internet use (at home / elsewhere) & 1.12 & .11 & -0.76 & -.05 \\
Working situation (inactive / active) & -0.31 & -.04 & -1.07 & -.09 \\
\hline$R^{2}$ & & .13 & & .23 \\
$F$ & & $2.82^{* * *}$ & & $4.67^{* * *}$ \\
\hline${ }^{*} p<.05,{ }^{* *} p<.01,{ }^{* * *} p<.001$ & & & &
\end{tabular}

Table 4.7 reveals that educational level of attainment is a significant contributor to the number of successfully completed strategic Internet skills tasks. As was the case with information Internet skills, none of the other variables appear as significant contributors. There are no significant contributors for the time spent on these tasks.

Table 4.7 Linear Regression on Number of Strategic Tasks Completed Successfully and Time Spent

\begin{tabular}{lcccc}
\hline Independent variables & \multicolumn{3}{c}{ Task completion } & \multicolumn{2}{c}{ Time spent } \\
& $t$ & $\beta$ & $t$ & $\beta$ \\
\hline Gender (M/F) & -0.72 & -.06 & -1.11 & -.11 \\
Age & -1.42 & -.17 & -0.19 & -.03 \\
Education (low-medium-high) & 4.24 & $.42^{\star * *}$ & 1.06 & .13 \\
Years online & 0.21 & .02 & 0.54 & .06 \\
Hours online weekly & -1.60 & -.15 & -1.23 & -.14 \\
Internet class (no / yes) & 0.31 & .03 & 0.47 & .05 \\
Assistance required (no / yes) & -1.61 & -.16 & 1.20 & .14 \\
Primary location of Internet use (at home / elsewhere) & -0.61 & -.05 & -0.26 & -.03 \\
Working situation (inactive / active) & 1.29 & .14 & -0.62 & -.08 \\
\hline$R^{2}$ & & .30 & & .01 \\
$F$ & & $6.09^{* * *}$ & & .84 \\
\hline${ }_{* \star *} p<.001$ & & & &
\end{tabular}




\subsection{Results of performance test 2: leisure information}

\subsubsection{General results}

As can be seen from Table 4.8, altogether, the subjects completed an average of $74 \%$ of the operational Internet skills tasks, $80 \%$ of the four formal Internet skills tasks, $53 \%$ of the information skills tasks, and $29 \%$ of the two strategic Internet skills tasks successfully. The time spent on the tasks again varies considerably for all four Internet skills. Regarding the two strategic Internet skills tasks, for example, the time ranged from a little over 7 minutes to 45 minutes.

Table 4.8 Overview of Successful Task completion and Time Spent

\begin{tabular}{lccrrr}
\hline Independent variables & \multicolumn{2}{c}{ Task completion } & \multicolumn{3}{c}{ Time spent (seconds) } \\
& M (SD) & $\%$ & M (SD) & Min. & Max. \\
\hline Operational tasks (8) & $5.9(1.9)$ & 74 & $427(198)$ & 118 & 980 \\
Formal tasks (4) & $3.2(1.0)$ & 80 & $450(218)$ & 180 & 1143 \\
Information tasks (3) & $1.6(0.9)$ & 53 & $960(336)$ & 343 & 1717 \\
Strategic tasks (2) & $0.6(0.7)$ & 29 & $1613(545)$ & 441 & $2500^{*}$ \\
\hline${ }^{*}$ maximum time allowed & & & & &
\end{tabular}

Table 4.9 Number of Tasks Subjects Failed to Complete Successfully

\begin{tabular}{lcc}
\hline & \# of failed tasks & \% of subjects \\
\hline Operational Internet skills & 0 & 25 \\
& 1 & 13 \\
2 & 16 \\
& 3 & 18 \\
& 4 & 8 \\
& 5 & 9 \\
& 6 & 6 \\
& 7 & 4 \\
Formal Internet skills & 8 & 0 \\
& 0 & 55 \\
& 1 & 22 \\
Information Internet skills & 2 & 16 \\
& 3 & 6 \\
& 4 & 0 \\
\hline Strategic Internet skills & 0 & 16 \\
& 1 & 45 \\
& 2 & 22 \\
& 3 & 17 \\
\hline
\end{tabular}


In Table 4.9, it is shown that $25 \%$ of the subjects were able to complete all operational Internet skills tasks, 55\% were able to complete all formal Internet skills tasks, 16\% were able to complete all information Internet skills tasks, and only $13 \%$ of the subjects were able to complete both the strategic skills tasks. Similar to the results of the first study, as much as $56 \%$ of the subjects could not complete either of the two strategic Internet skills tasks successfully. This was $17 \%$ of the subjects regarding the three information Internet skills tasks. As expected, both strategic Internet skills tasks were the hardest and both could only be completed successfully by $29 \%$ of the subjects. One of these tasks, for example, asked the subjects whether it would be cheaper to go to Amsterdam under specific conditions by train or by car. The hardest information Internet skills task was completed by $44 \%$ of the subjects and provided the subjects with the task of finding out whether they are allowed to use a rubber boat of 9.84 feet in length with a maximum speed of $17 \mathrm{mph}$ in public waters.

\subsubsection{Internet skills determinants}

As shown in Table 4.10, age is the strongest contributor to the level of operational Internet skills. Age has the highest betas, significant both for the number of tasks completed successfully and the time spent on the tasks. Educational level of attainment appears as a significant contributor to the number of successfully completed tasks. Internet experience and amount of Internet use have a significant effect on the time spent on the operational Internet skill tasks. Finally, using the Internet primarily at home contributes to the number of operational assignments completed successfully.

Table 4.10 Linear Regression on Number of Operational Tasks Completed Successfully and Time Spent

\begin{tabular}{lcccc}
\hline Independent variables & \multicolumn{2}{c}{ Task completion } & \multicolumn{2}{c}{ Time spent } \\
& $t$ & $\beta$ & $t$ & $\beta$ \\
\hline Gender (M/F) & -1.01 & -.07 & 0.87 & .05 \\
Age & -5.24 & $-.50^{\star \star *}$ & 6.77 & $.57^{\star \star \star}$ \\
Education (low-medium-high) & 2.77 & $.20^{\star *}$ & -0.92 & -.06 \\
Years online & 0.43 & .03 & -3.22 & $-.20^{\star \star}$ \\
Hours online weekly & 1.80 & .15 & -2.02 & $-.15^{\star}$ \\
Internet class (no / yes) & -0.53 & -.04 & 1.00 & .06 \\
Assistance required (no / yes) & -0.94 & -.07 & 1.66 & .11 \\
Primary location of Internet use (at home / elsewhere) & -2.28 & $-.16^{\star}$ & 0.62 & .04 \\
Working situation (inactive / active) & -1.23 & -.11 & 1.02 & .08 \\
\hline$R^{2}$ & & .55 & & .65 \\
$F$ & & $15.46^{* \star *}$ & & $23.09^{* \star *}$ \\
\hline${ }^{*} p<.05,{ }^{* *} p<.01,{ }^{* \star *} p<.001$ & & & &
\end{tabular}


Regarding formal Internet skills, again, age appears as a significant contributor to the number of completed tasks successfully and the time spent. In addition, educational level of attainment appears significant for the number of formal tasks completed successfully. The primary location of Internet use also plays a significant role on the number of successfully completed formal Internet skill tasks and time spent. Finally, increased Internet experience leads to less time being spent on the operational assignments (see Table 4.11).

Table 4.11 Linear Regression on Number of Formal Tasks Completed Successfully and Time Spent

\begin{tabular}{|c|c|c|c|c|}
\hline \multirow[t]{2}{*}{ Independent variables } & \multicolumn{2}{|c|}{ Task completion } & \multicolumn{2}{|c|}{ Time spent } \\
\hline & $t$ & $\beta$ & $t$ & $\beta$ \\
\hline Gender (M/F) & 1.22 & .09 & -0.71 & -.05 \\
\hline Age & -3.41 & $-.35^{\star \star *}$ & 5.08 & $.48^{\star \star \star}$ \\
\hline Education (low-medium-high) & 3.49 & $.27^{\star \star *}$ & -1.50 & -.11 \\
\hline Years online & 2.66 & .21 & -2.31 & $-.17^{\star}$ \\
\hline Hours online weekly & -0.23 & -.02 & -1.58 & -.13 \\
\hline Internet class (no / yes) & 1.30 & .10 & -1.63 & -.11 \\
\hline Assistance required (no / yes) & -0.41 & -.03 & 1.24 & .10 \\
\hline Primary location of Internet use (at home / elsewhere) & -3.19 & $-.24^{\star \star}$ & 2.73 & $.19^{\star *}$ \\
\hline Working situation (inactive / active) & -1.79 & -.17 & 1.02 & .09 \\
\hline$R^{2}$ & & .51 & & .55 \\
\hline$F$ & & $11.36^{\star * *}$ & & $15.64^{* \star *}$ \\
\hline
\end{tabular}

Table 4.12 Linear Regression on Number of Information Tasks Completed Successfully and Time Spent

\begin{tabular}{lcccc}
\hline Independent variables & \multicolumn{2}{c}{ Task completion } & \multicolumn{2}{c}{ Time spent } \\
& $t$ & $\beta$ & $t$ & $\beta$ \\
\hline Gender (M/F) & -1.05 & -.10 & 1.58 & .15 \\
Age & -0.42 & -.05 & 2.45 & $.31^{*}$ \\
Education (low-medium-high) & 2.95 & $.28^{* * *}$ & 1.60 & .16 \\
Years online & 1.36 & .13 & -2.55 & -.25 \\
Hours online weekly & 0.28 & .03 & 0.17 & .02 \\
Internet class (no / yes) & -0.17 & -.02 & 1.78 & .17 \\
Assistance required (no / yes) & -2.07 & $-.21^{*}$ & 1.04 & .11 \\
Primary location of Internet use (at home / elsewhere) & -0.90 & -.08 & 0.52 & -.05 \\
Working situation (inactive / active) & -0.50 & -.06 & 0.28 & .03 \\
\hline$R^{2}$ & & .27 & & .18 \\
$F$ & & $3.99^{* * *}$ & & $3.55^{* \star *}$ \\
${ }^{*} p<.05,{ }^{* *} p<.001$ & & & &
\end{tabular}

The regression results presented in Table 4.12 reveal that educational level of attainment is a significant contributor to the number of successfully completed information tasks. Age appears as a significant contributor to the time spent on these tasks. Finally, assistance required is significant for the number of tasks 
completed successfully. The less likely people are to need help when using the Internet, the more information Internet skills tasks they successfully complete.

Table 4.13 shows that educational level of attainment is the only significant contributor to the number of successfully completed strategic Internet skills tasks. There are no significant contributors for the time spent on these tasks.

Table 4.13 Linear Regression on Number of Strategic Tasks Completed Successfully and Time Spent

\begin{tabular}{lcccc}
\hline Independent variables & \multicolumn{2}{c}{ Task completion } & \multicolumn{2}{c}{ Time spent } \\
& $t$ & $\beta$ & $t$ & $\beta$ \\
\hline Gender (M/F) & -1.06 & -.10 & 1.35 & .14 \\
Age & 0.15 & .02 & 0.14 & .02 \\
Education (low-medium-high) & 4.31 & $.42^{* * *}$ & 1.26 & .14 \\
Years online & 0.64 & .06 & 0.96 & .10 \\
Hours online weekly & -0.48 & -.06 & 0.26 & .03 \\
Internet class (no / yes) & -0.11 & -.01 & 2.02 & .21 \\
Assistance required (no / yes) & -1.14 & -.12 & 0.57 & .07 \\
Primary location of Internet use (at home / elsewhere) & -0.94 & -.09 & -0.58 & -.06 \\
Working situation (inactive / active) & -0.58 & -.07 & -0.15 & -.02 \\
\hline$R^{2}$ & & .25 & & .09 \\
$F$ & & $3.75^{\star * *}$ & & 1.07 \\
\hline${ }^{*} p<.05,{ }^{* *} p<.01,{ }^{* * *} p<.001$ & & & &
\end{tabular}

\subsection{Results of performance test 3: Health information}

\subsubsection{General results}

The subjects successfully completed an average of $73 \%$ of the eight operational Internet skills tasks and an average of $73 \%$ of the four formal Internet skills tasks. Of the three information Internet skills tasks, an average of 50\% was completed successfully, and of the two strategic Internet skills tasks, an average of $35 \%$. Again, the time spent on all the assignments varies substantially.

Table 4.14 Overview of Successful Task Completion and Time Spent

\begin{tabular}{lccrrr}
\hline Independent variables & \multicolumn{2}{c}{ Task completion } & \multicolumn{3}{c}{ Time spent (seconds) } \\
& M (SD) & $\%$ & M (SD) & Min. & Max. \\
\hline Operational tasks (8) & $5.8(2.1)$ & 73 & $427(198)$ & 118 & 980 \\
Formal tasks (4) & $2.9(1.2)$ & 73 & $450(218)$ & 180 & 1143 \\
Information tasks (3) & $1.5(0.9)$ & 50 & $960(336)$ & 343 & 1717 \\
Strategic tasks (2) & $0.7(0.8)$ & 35 & $1613(545)$ & 441 & 2500 \\
\hline
\end{tabular}


In Table 4.15 , it is shown that $28 \%$ of the subjects were able to complete all operational Internet skills tasks, 39\% were able to complete all formal Internet skills tasks, only 13\% were able to complete all information Internet skills tasks, and $20 \%$ of the subjects were able to complete both the strategic skills tasks. Forty-four percent of the subjects could not complete either of the two strategic Internet skills tasks successfully. This was $17 \%$ of the subjects regarding the three information Internet skills tasks. The second strategic Internet skills tasks was the hardest and could only be completed successfully by $25 \%$ of the subjects. In this task, subjects were asked to find a homecare organization in the city of Enschede with a special caring program for individuals suffering from dementia and impaired hearing. The hardest information Internet skills task was completed by only $28 \%$ of the subjects and asked the subjects whether it is a good idea to start an antiviral (remedy against viral infections) for Lyme borreliosis.

Table 4.15 Number of Tasks the Subjects Failed to Complete Successfully

\begin{tabular}{lcc}
\hline & \# of failed tasks & \% of subjects \\
\hline Operational Internet skills & 0 & 28 \\
& 1 & 21 \\
2 & 16 \\
& 3 & 10 \\
& 4 & 10 \\
& 5 & 9 \\
& 6 & 4 \\
& 7 & 3 \\
Formal Internet skills & 8 & 0 \\
& 0 & 39 \\
& 1 & 28 \\
Information Internet skills & 2 & 17 \\
& 3 & 11 \\
& 4 & 5 \\
\hline Strategic Internet skills & 0 & 13 \\
& 1 & 40 \\
& 2 & 34 \\
& 3 & 14 \\
\hline
\end{tabular}




\subsubsection{Internet skills determinants}

Table 4.16 shows that age and education are the two significant contributors to the number of operational Internet skills tasks successfully completed and to the time spent on these tasks. Age is the strongest contributor.

Table 4.16 Linear Regression on Number of Operational Tasks Completed Successfully and Time Spent

\begin{tabular}{lcccc}
\hline Independent variables & \multicolumn{2}{c}{ Task completion } & \multicolumn{2}{c}{ Time spent } \\
& $t$ & $\beta$ & $t$ & $\beta$ \\
\hline Gender (M/F) & -1.21 & -.10 & -0.31 & -.02 \\
Age & -6.99 & $-.71^{* \star *}$ & 4.89 & $.51^{\star \star *}$ \\
Education (low-medium-high) & 2.04 & $.18^{\star}$ & -2.94 & $-.27^{* \star}$ \\
Years online & 1.39 & .12 & -1.71 & -.15 \\
Hours online weekly & 0.18 & .02 & 0.42 & -.04 \\
Internet class (no / yes) & 0.27 & .02 & 1.22 & .10 \\
Assistance required (no / yes) & 0.34 & .03 & 0.92 & .08 \\
Primary location of Internet use (at home / elsewhere) & 0.17 & .01 & -1.06 & -.09 \\
Working situation (inactive / active) & -1.11 & -.11 & -0.53 & -.06 \\
\hline$R^{2}$ & & .55 & & .54 \\
$F$ & & $10.11^{* \star *}$ & & $10.11^{* * *}$ \\
\hline${ }^{*} p<.05,{ }^{* *} p<.01,{ }^{* * *} p<.001$ & & & &
\end{tabular}

Table 4.17 reveals that regarding formal Internet skills, again, age and education are significant contributors to both the number of successfully completed tasks and the time spent. In addition, years of Internet experience also contributes significantly to both equations.

Table 4.17 Linear Regression on Number of Formal Tasks Completed Successfully and Time Spent

\begin{tabular}{|c|c|c|c|c|}
\hline \multirow[t]{2}{*}{ Independent variables } & \multicolumn{2}{|c|}{ Task completion } & \multicolumn{2}{|c|}{ Time spent } \\
\hline & $t$ & $\beta$ & $t$ & $\beta$ \\
\hline Gender (M/F) & -1.19 & -.09 & -0.07 & .00 \\
\hline Age & -6.69 & $-.62^{\star \star \star}$ & 7.10 & $.61^{\star \star *}$ \\
\hline Education (low-medium-high) & 4.85 & $.40^{\star \star \star}$ & -4.54 & $-.34^{\star \star \star}$ \\
\hline Years online & 2.12 & $.17^{\star}$ & -2.10 & $-.15^{\star}$ \\
\hline Hours online weekly & -1.40 & -.11 & 0.01 & .00 \\
\hline Internet class (no / yes) & -0.63 & -.05 & 0.99 & .07 \\
\hline Assistance required (no / yes) & -0.81 & -.06 & 0.36 & .03 \\
\hline Primary location of Internet use (at home / elsewhere) & 0.97 & .07 & -0.54 & -.04 \\
\hline Working situation (inactive / active) & -1.37 & -.13 & -0.20 & -.02 \\
\hline$R^{2}$ & & .61 & & .68 \\
\hline$F$ & & $13.60^{\star * *}$ & & $18.20^{* * *}$ \\
\hline
\end{tabular}


The regression results reported in Table 4.18 reveal that educational level of attainment is the strongest significant contributor to the number of information tasks completed successfully. Furthermore, participation in an Internet course positively contributes to the number of tasks completed successfully, and requiring assistance significantly and negatively contributes to the time spent on the tasks.

Table 4.18 Linear Regression on Number of Information Tasks Completed Successfully and Time Spent

\begin{tabular}{lcccc}
\hline Independent variables & \multicolumn{2}{c}{ Task completion } & \multicolumn{2}{c}{ Time spent } \\
& $t$ & $\beta$ & $t$ & $\beta$ \\
\hline Gender (M/F) & 1.00 & .09 & -1.19 & -.13 \\
Age & 0.46 & -.06 & 0.59 & .08 \\
Education (low-medium-high) & 5.29 & $.56^{\star * *}$ & -0.69 & -.08 \\
Years online & 0.65 & .01 & -0.67 & -.08 \\
Hours online weekly & -0.07 & -.01 & 0.02 & .00 \\
Internet class (no / yes) & -2.50 & $-.24^{*}$ & -0.04 & -.00 \\
Assistance required (no / yes) & -0.40 & -.04 & 2.43 & $.28^{\star}$ \\
Primary location of Internet use (at home / elsewhere) & -0.89 & -.09 & -0.49 & -.05 \\
Working situation (inactive / active) & -0.37 & -.00 & -0.30 & -.04 \\
\hline$R^{2}$ & & .34 & & .09 \\
$F$ & & $4.48^{\star * *}$ & & 1.70 \\
\hline${ }^{*} p<.05{ }^{* * *} p<001$ & & & &
\end{tabular}

${ }^{*} p<.05,{ }^{* \star *} p<.001$

Regarding strategic Internet skills, educational level of attainment is the only significant contributor to the number of successfully completed tasks (see Table 4.19). No other variables appeared significant.

Table 4.19 Linear Regression on Number of Strategic Tasks Completed Successfully and Time Spent

\begin{tabular}{lcccc}
\hline Independent variables & \multicolumn{2}{c}{ Task completion } & \multicolumn{2}{c}{ Time spent } \\
& $t$ & $\beta$ & $t$ & $\beta$ \\
\hline Gender (M/F) & 1.31 & .11 & 0.93 & .11 \\
Age & 0.06 & .01 & 1.13 & .17 \\
Education (low-medium-high) & 5.99 & $.58^{\star * *}$ & 0.87 & .11 \\
Years online & 0.76 & .07 & -1.15 & -.14 \\
Hours online weekly & 0.31 & .03 & 0.16 & .02 \\
Internet class (no / yes) & -0.58 & -.05 & -0.52 & -.06 \\
Assistance required (no / yes) & -0.01 & -.00 & -0.27 & -.03 \\
Primary location of Internet use (at home / elsewhere) & 0.26 & .02 & -1.08 & -.13 \\
Working situation (inactive / active) & 1.06 & .12 & 0.66 & .10 \\
\hline$R^{2}$ & & .45 & & .07 \\
$F$ & & $7.10^{\star * *}$ & & 0.62 \\
\hline${ }^{*} p<.05,{ }^{* *} p<.01,{ }^{* * *} p<.001$ & & & &
\end{tabular}




\subsection{Modeling Internet skills determinants}

Table 4.20 Significant Contributors (s) to the number of assignments completed successfully (\#tasks) and the time spent (Time) for all Four Internet Skills in Studies 1, 2, and 3

\begin{tabular}{|c|c|c|c|c|c|c|}
\hline & \multicolumn{2}{|c|}{ Study 1} & \multicolumn{2}{|c|}{ Study 2} & \multicolumn{2}{|c|}{ Study 3} \\
\hline & \# tasks & Time & \# tasks & Time & \# tasks & Time \\
\hline \multicolumn{7}{|l|}{ Operational Internet skills } \\
\hline Gender & - & - & - & - & - & - \\
\hline Age & $s$ & $s$ & $s$ & $s$ & $s$ & $s$ \\
\hline Education & $s$ & $s$ & $s$ & - & $s$ & $s$ \\
\hline Years online & $s$ & $s$ & - & $s$ & - & - \\
\hline Hours online weekly & - & - & - & $s$ & - & - \\
\hline Internet class & - & - & - & - & - & - \\
\hline Assistance required & - & - & - & - & - & - \\
\hline Primary location of Internet use & - & - & $s$ & - & - & - \\
\hline Working situation & - & $s$ & - & - & - & - \\
\hline \multicolumn{7}{|l|}{ Formal Internet skills } \\
\hline Gender & - & - & - & - & - & - \\
\hline Age & $s$ & $s$ & $s$ & $s$ & $s$ & $s$ \\
\hline Education & $s$ & $\mathrm{~s}$ & $\mathrm{~s}$ & - & $\mathrm{s}$ & s \\
\hline Years online & - & - & - & $s$ & $\mathrm{~s}$ & $\mathrm{~s}$ \\
\hline Hours online weekly & - & - & - & - & - & - \\
\hline Internet class & - & - & - & - & - & - \\
\hline Assistance required & $s$ & - & - & - & - & - \\
\hline Primary location of Internet use & $s$ & - & $s$ & $s$ & - & - \\
\hline Working situation & - & - & - & - & - & - \\
\hline \multicolumn{7}{|l|}{ Information Internet skills } \\
\hline Gender & - & - & - & - & - & - \\
\hline Age & - & $s$ & - & $s$ & - & - \\
\hline Education & $s$ & s & $s$ & - & s & - \\
\hline Years online & - & - & - & - & - & - \\
\hline Hours online weekly & - & - & - & - & - & - \\
\hline Internet class & - & - & - & - & - & - \\
\hline Assistance required & - & - & s & - & - & s \\
\hline Primary location of Internet use & - & - & - & - & - & - \\
\hline Working situation & - & - & - & - & - & - \\
\hline \multicolumn{7}{|l|}{ Strategic Internet skills } \\
\hline Gender & - & - & - & - & - & - \\
\hline Age & - & - & - & - & - & - \\
\hline Education & s & - & s & - & s & - \\
\hline Years online & - & - & - & - & - & - \\
\hline Hours online weekly & - & - & - & - & - & - \\
\hline Internet class & - & - & - & - & - & - \\
\hline Assistance required & - & - & - & - & - & - \\
\hline Primary location of Internet use & - & - & - & - & - & - \\
\hline Working situation & - & - & - & - & - & - \\
\hline
\end{tabular}

Table 4.20 overviews all significant contributors to the four Internet skills in all three studies. From this table, it appears that age, education, and Internet experience are the most important variables to predict the levels of the four 
Internet skills. As a following step, the exact relationship between these variables and Internet skills is further assessed using multivariate methods. Furthermore, since the number of hours spent online is often considered an important factor in the public debate, this variable is added to the analyses.

Structural equation modeling was undertaken using Amos 17.0 (Arbuckle, 2010). Analyses are based on maximum likelihood estimation, with all data being treated as shaping continuous scale. As suggested by Holbert and Stephenson (2002), the following model fit indices are used: The Chi-square estimate with degrees of freedom, given that it is still the most commonly used means by which to make comparisons across models (Hoyle \& Panter, 1995). The ratio between Chi-square and degrees of freedom should not exceed 5 for models with a good fit (Bentler, 1989). Additionally, the standardized root mean squared residual (SRMR) as a second absolute fit statistic (Hu \& Bentler, 1999) in combination with the TuckerLewis index (TLI) as incremental index and the root mean squared error of approximation (RMSEA) (Browne \& Cudeck, 1993) are reported. Hu and Bentler recommend using a cutoff value close to .95 for TLI in combination with a cutoff value close to .09 for SRMR to evaluate model fit and the RMSEA close to .06 or less.

When applying SEM, sample size should be large, as it relies on tests that are sensitive to sample size as well as to the magnitude of differences in covariance matrices. Schumacker and Lomax (2004) surveyed the literature and found sample sizes of 250 to 500 to be used in "many articles" and "numerous studies . . . that were in agreement" that fewer than 100 or 150 subjects was below the minimum. One rule of thumb found in the literature is that sample size should be at least 50 more than 8 times the number of variables in the model. Mitchell (1993) advances the rule of thumb that there be 10 to 20 times as many cases as variables. Another rule of thumb, based on Stevens (1996), is to have at least 15 cases per measured variable or indicator. To obtain a sufficient sample size, in all three performance tests, the average successful completion of the tasks are standardized and subsequently merged for all four types of Internet skills. This is allowed because the measurement of the skills in all studies was the same except for the content on which they focus (i.e., government, leisure, health). The aggregate of three samples comprises a sample of 306 subjects that actually performed assignments on the Internet. During the SEM analyses in AMOS, Hoelter's critical $\mathrm{N}$ is issued to judge if the applied sample size is adequate. By convention, sample size is 


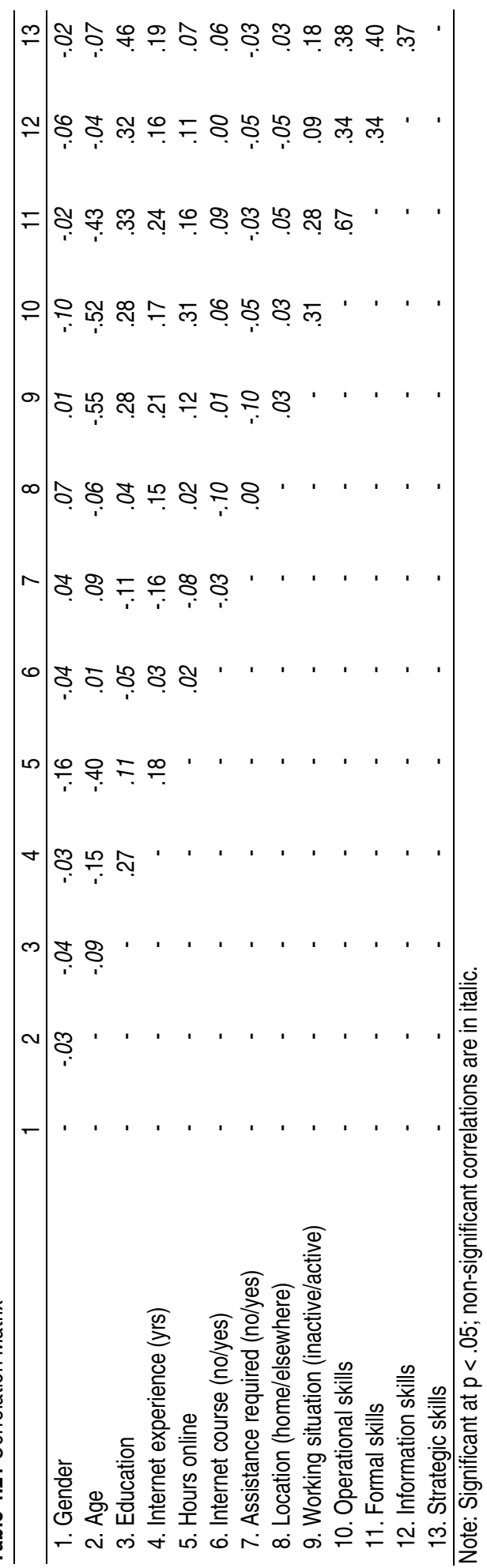


adequate if Hoelter's $\mathrm{N}>200$. A Hoelter's $\mathrm{N}$ under 75 is considered too low to accept a model by Chi-square. The eventual model, as presented in Figure 4.2, resulted in a Hoelter's N of 333 (at the .05 levels of significance) and 415 (at the .01 levels of significance).

Two dependent latent variables are constructed, based on the distinction between medium- and content-related Internet skills. The two latent variables are not measured directly, but are estimated from the related measured variables. Operational and formal Internet skills "tap" into the latent variables together labeled as medium-related Internet skills, and information and strategic Internet skills into the latent variables together labeled as content-related Internet skills. A complete overview of all variables accounted for in the prior analyses is shown in Table 4.21. From this table, it appears that the correlations between gender, location of Internet use, following an Internet course, and the four Internet skills are not significant. Furthermore, the correlation between requiring assistance when using the Internet and operational and strategic Internet skills is significant, but very low. These variables are excluded from the path analysis. The model with the variables of age, education, Internet experience, hours spent online, and the medium- and content-related Internet skills is presented in Figure 4.1.

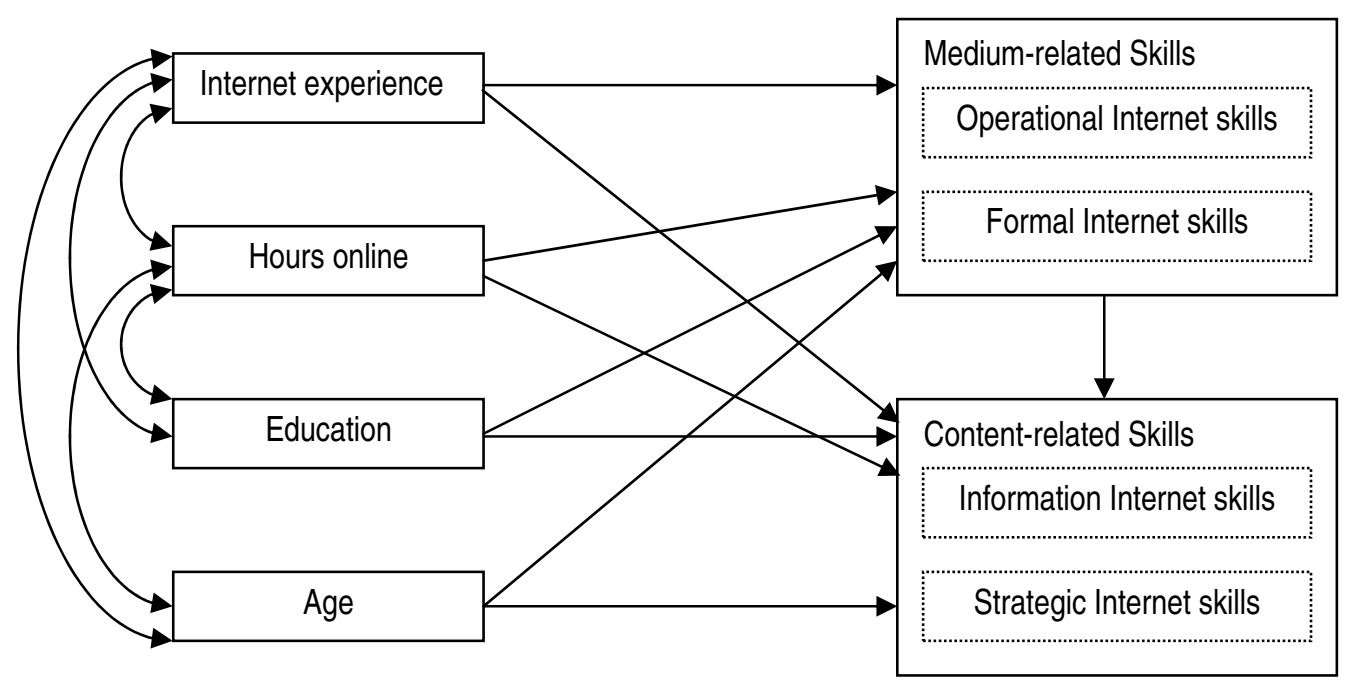

Figure 4.1 Model with Internet experience, Hours Online, Education, and Age as Independent Variables and Medium- and Content-related Internet Skills as Dependent Variables. 
To test the sequential and conditional nature of medium-related Internet and content-related Internet skills, two path analyses were conducted. One path analysis involved a direct path from medium-related skills to content-related skills, while the other path analysis did not include a direct path. The results obtained by testing the validity of a causal structure with a direct path from medium-related skills to content-related skills showed a good fit, $\chi^{2}(11)=19.01, \chi^{2} / \mathrm{df}=1.73$, SRMR $=.032$, TLI $=.972$, RMSEA $=.049$ (90\% confidence interval $[\mathrm{C}]: .000$, $.085), \mathrm{AIC}=69.013, \mathrm{ECVI}=.226$ (CI: .200, .279). The results obtained by testing the validity of a causal structure without a direct path from medium-related skills to content-related skills showed a poor fit, $\chi^{2}(12)=44.38, \chi^{2} / \mathrm{df}=3.70$, SRMR $=.055$, TLI $=.895$, RMSEA $=.094(90 \%$ confidence interval $[\mathrm{CI}]: .065, .124)$, $\mathrm{AIC}=92.381, \mathrm{ECVI}=.303$ (CI: .248, .383).

To test whether Internet experience and amount of Internet use also influence the content-related Internet skills, an additional path analysis was conducted without the direct paths from Internet experience and Internet amount to content-related skills. The results obtained by testing the validity of a causal structure without the direct paths from Internet experience and hours online to content-related skills showed an improved fit, $\chi^{2}(12)=19.31, \chi^{2} / \mathrm{df}=1.61, \mathrm{SRMR}=.033$, TLI $=$ .976, RMSEA $=.045(90 \%$ confidence interval [CI]: .000, .080), $\mathrm{AIC}=67.311$, $\mathrm{ECVI}=.221$ (CI: .197, .274). Squared multiple correlations provide information about the variance accounted for by the complete set of variables and show that medium-related skills accounted for 69\% (operational Internet skills for 69\% and formal Internet skills for 63\%) and content-related skills accounted for $80 \%$ (information Internet skills for 31\% and strategic Internet skills for 44\%).

The path model with standardized path coefficients is featured in Figure 4.2. The standardized path coefficients show a significant direct effect of Internet experience, education, and age on medium-related skills. The direct effect of hours spent online weekly on medium-related skills is not significant. Figure 4.2 also shows significant direct effects of education, age, and medium-related skills on content-related skills.

The results from Figure 4.2 further strengthen the results obtained from the linear regression analyses in all three studies. Higher age appears as a negative contributor to the medium-related skills (i.e., operational and formal Internet skills); the younger generations perform better on these skills. However, the 


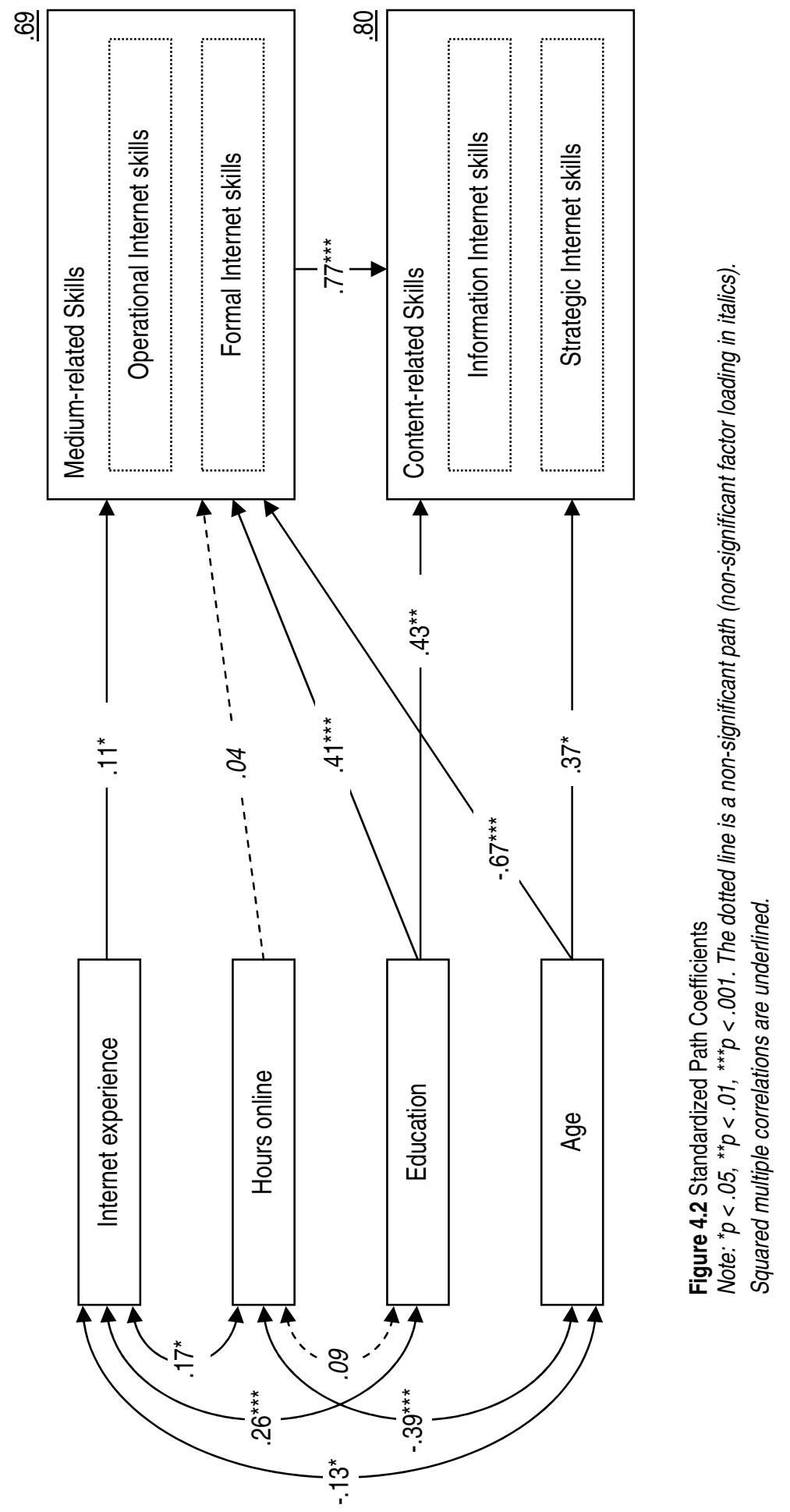


results regarding content-related skills prove different. In fact, it seems that higher age positively contributes to the level of content-related skills, meaning that older generations perform better than younger ones. Educational attainment appears significant both for the medium and content-related Internet skills. Internet experience only contributes to the medium-related skills. It appears that contentrelated skills do not grow with years of Internet experience or amount of time spent online weekly. Amount of Internet use only has a slight effect on mediumrelated skills.

From the measurement model presented in Figure 4.2, it appears that age is a significant contributor to the medium-related Internet skills (i.e., operational and formal Internet skills). The model also reveals that age negatively contributes to the level of content-related skills, indicating that younger people have to be taught better information skills and the appropriate strategic skills in particular domains. Conversely, elderly people have to learn medium-related skills more effectively. In fact, there is a direct, positive, significant effect of age on content-related skills, meaning that the older generations perform better than the younger with regard to these skills. However, the final results of the performance tests measuring both medium- and content-related skills are still negative. The direct effect of age on content-related Internet skills is mediated by the indirect effect of age on mediumrelated skills. When the path from age- to medium-related Internet skills $(\beta=-.67)$ is multiplied by the path from medium-related skills to content-related skills $(\beta=$ $.77)$ and summed with the direct path from age to content-related Internet skills ( $\beta$ $=.37$ ), the result is a $\beta$ of -.15 . This indicates that the medium-related skills have a major influence on the performance of content-related-skills, so that the older generations still score slightly lower on performance tests than young people with regard to these skills. This conclusion further strengthens the importance of measuring Internet skills comprehensively and in detail, considering the full range of Internet skills.

Educational attainment appears positively related to both medium- and contentrelated Internet skills. The level of educational attainment affects both mediumand content-related Internet skills. The total effect of education on content-related skills $(\beta=.75)$ results from the direct effect of education on content-related skills added by the indirect effect of education on medium-related skills. It appears that content-related skills do not grow with years of Internet experience or amount of 
time spent online weekly. Internet experience only has a minor effect $(\beta=.11)$ on medium-related Internet skills.

\subsection{Evaluating the Internet Skills definition}

The results of the three performance tests reveal the independent contribution of several contributors to the four types of Internet skills. Age and educational level of attainment appear most important. The importance of age and education in all types of access to digital technology is observed in almost every empirical study of the digital divide. Comparable results regarding Internet skills are observed in the performance tests conducted by Hargittai (2002) in the United States, although this test did not account for the four different Internet skills as measured in the studies reported in this dissertation. Here, age appeared to be a significant negative contributor to the level of operational and formal Internet skills. The level of educational attainment appeared significant to the levels of operational, formal, information, and strategic Internet skills.

The level of Internet skills measured appeared to have a weak relation with years of Internet experience and number of hours spent online weekly. Internet experience contributed to the level of operational Internet skills, but formal, information, and strategic Internet skills did not grow with years of Internet experience or amount of time spent online. This goes against the predominant public opinion. However, it is in line with other research that reports that measures of experience are poor predictors of ability (e.g., Chadwick-Dias, McNulty, \& Tullis, 2003).

In the following evaluation of the performance results, it will be claimed that the proposed Internet skill definition is appropriate to measure Internet skills. At the very least, it is better than other methods used to measure these skills. When the findings of the performance tests are compared with conclusions drawn from survey research, it appears that these surveys provide an overly flattering picture. They often only focus on operational and formal skills, or they ask Internet skillrelated questions that are too general or superficial. They tend to ask for general opinions about the level of skills instead of specific questions about actual behavior (achievements in computer and Internet use). The proposed framework 
for measuring Internet skills could be a powerful means for understanding the complexity of the Internet skills that people employ when they use the Internet.

The measurement of the framework is practicable though rather laborious. The performance tests themselves require 1.5 hours for every subject. In-depth analysis of the results lasts equally long. Preparation and closing of the test (sampling, reception of subjects, filling in questionnaires) takes approximately a half hour per subject. This whole procedure makes this kind of measurement very costly in terms of time and money. It is the main reason why no more than approximately one hundred people can be tested in each study. Because of this, researchers in this field are looking for equally reliable and valid alternatives. The most attractive candidate is posing focused proxy questions in surveys. These questions ask for reports of behavior that indicates a particular Internet skill. In Chapter 6, these questions are further discussed and a measurement instrument to be used in surveys is proposed.

For a legitimate evaluation of the reliability and validity of the developed and tested Internet skill definition, it is important to realize that test development in this field is still in an exploratory stage. As of yet, there are no generally accepted touchstones or criteria for measuring Internet skills.

\subsubsection{Reliability}

The reliability was safeguarded by equalizing all experimental conditions in the three performance tests. Intra-observation reliability was not relevant because all dimensions and indices of the Internet skills definition were measured by a single assignment. Adding assignments to each item of the already extensive performance tests of 1.5 hours was unrealistic. Inter-observation reliability was at stake, as the three-test series used exactly the same kind of assignments for a particular skill, only differing in topic (government, leisure, and health information). The fact that the three studies produced the same results improved reliability and validity. Motivation or interest for these topics would not have to be conceived as a disturbing factor.

In Table 4.23, the means of tasks completed successfully in all three studies are compared. None of the independent sample $t$-tests appear significant, indicating 
that the means do not differ. The percentages of completed assignments belonging to a specific skill follow the same pattern in both studies. Indeed, the conclusions of all three studies agree and can be considered mutually supportive. More important, however, is the result that all studies put forward similar contributors to the four Internet skill levels.

Table 4.23 F-values of Comparison between the Means of the Number of Successfully Completed Operational (O), Formal (F), Information (I), and Strategic (S) Internet Skills in Studies (s) 1, 2, and 3

\begin{tabular}{lrrrrrrrr}
\hline & $\mathrm{O}(\mathrm{s} 1)$ & $\mathrm{O}(\mathrm{s} 3)$ & $\mathrm{F}(\mathrm{s} 1)$ & $\mathrm{F}(\mathrm{s} 3)$ & $\mathrm{I}(\mathrm{s} 1)$ & $\mathrm{I}(\mathrm{s} 3)$ & $\mathrm{S}(\mathrm{s} 1)$ & $\mathrm{S}(\mathrm{s} 3)$ \\
\hline $\mathrm{O}(\mathrm{s} 1)$ & - & 0.00 & - & - & - & - & - & - \\
$\mathrm{O}(\mathrm{s} 2)$ & 0.04 & 0.03 & - & - & - & - & - & - \\
$\mathrm{F}(\mathrm{s} 1)$ & - & - & - & 0.26 & - & - & - & - \\
$\mathrm{F}(\mathrm{s} 2)$ & - & - & 0.34 & 0.00 & - & - & - & - \\
$\mathrm{I}(\mathrm{s} 1)$ & - & - & - & - & - & 0.02 & - & - \\
$\mathrm{I}(\mathrm{s} 2)$ & - & - & - & - & 2.10 & 2.21 & - & - \\
$\mathrm{S}(\mathrm{s} 1)$ & - & - & - & - & - & - & - & 0.11 \\
$\mathrm{~S}(\mathrm{~s} 2)$ & - & - & - & - & - & - & 0.42 & 0.04 \\
\hline
\end{tabular}

Note: None of the F-values appeared significant

\subsubsection{Validity}

For evaluating the validity of the framework, the official 1999 Standards for Educational and Psychological Testing (American Educational Research Association et al., 1999) that are based on the modern model of validity designed by Messick (1995) are followed. Messick proposed a single construct of validity using several types of evidence (content, construct, and criterion). Content-related evidence of validity can be found when the measure concerned represents all known facets of the concept or domain under investigation. The literature overview in Chapter 3 has made an inventory of several known theoretical and operational definitions of Internet skills. The proposed definition directly emerged from this inventory-including the attempt to make a well-founded selectionminimizing the chance that the definition falls outside the accepted body of knowledge in this field of investigation.

Construct-related evidence of validity refers to the empirical and theoretical support for the construct under investigation (i.e. Internet skills). The empirical phenomena that belong to the parts of this construct according to Internet skills theory are searched for in Chapter 3. The proposed definition consisted of a medium-related skills part (operational and formal skills) and a content-related 
skills part (information and strategic skills) with a particular coherence that is expected to have a conditional nature according to the theory. In both studies, the percentages of completed medium skill-related assignments are higher than the percentages of completed content skill-related assignments. In addition, no subjects completed any of the content-related skill assignments without also having completed medium-related skill assignments. The opposite is the case: there are subjects that did not complete any of the content-related skill assignments, but did complete medium skill-related assignments. The path from medium and content-related skills in Figure 4.2 appeared very high $(\beta=.77)$. This also indicates that the relation between the medium and content-related skills is characterized by a conditional nature. Thus, the framework is considered valid in this respect.

Construct validity also means that the definition as a whole agrees to a certain extent with other constructs or measures of Internet skills. Currently, there are three competitive measures: indirect measures of Internet skills (years of Internet experience and Internet usage time), self-assessments in surveys, and performance tests. One might expect some correlation between the results of these measures; otherwise, one or more of them would seem invalid. This type of construct validity is called convergent validity. It is assumed that there will be a modest correlation between these measures but no absence of correlation and no very high correlation either. Although Cohen (1988) argued that criteria for the interpretation of a correlation coefficient are in some ways arbitrary, he considers a correlation between .5 and 1 large, between .3 and .5 medium, and under .3 small. Table 4.24 reveals that Internet experience correlates positively-mostly with a medium to small strength-with the number of completed skill assignments of all four skills. Amount of Internet use correlates mainly with the medium-related skills, but also only little.

Table 4.24 Pearson Correlations between Internet Experience and Hours Online with Number of Successfully Completed Operational, Formal, Information, and Strategic Skills Tasks

\begin{tabular}{|c|c|c|c|c|c|}
\hline & & $\begin{array}{l}\text { Operational } \\
\text { skills }\end{array}$ & $\begin{array}{l}\text { Formal } \\
\text { skills }\end{array}$ & $\begin{array}{l}\text { Information } \\
\text { skills }\end{array}$ & $\begin{array}{c}\text { Strategic } \\
\text { skills }\end{array}$ \\
\hline Internet experience & Study 1 & $.50^{\star \star}$ & $.46^{\star \star}$ & $.26^{* *}$ & $.32^{* \star}$ \\
\hline Hours online & Study 1 & $.33^{\star *}$ & $.27^{\star *}$ & Ns & Ns \\
\hline Internet experience & Study 2 & $.19^{\star *}$ & $.34^{* *}$ & $.28^{*}$ & $.20^{\star}$ \\
\hline Hours online & Study 2 & $.47^{\star \star}$ & $.24^{\star *}$ & $.24^{\star \star}$ & Ns \\
\hline Internet experience & Study 3 & $.20^{\star *}$ & $.28^{\star *}$ & $.16^{\star *}$ & $.19^{\star *}$ \\
\hline Hours online & Study 3 & $.33^{\star *}$ & $.26^{\star *}$ & $.11^{\star *}$ & Ns \\
\hline
\end{tabular}


In a survey prior to the performance tests, subjects were asked to estimate their own level of Internet skills on a five-point scale. In the first performance test, the mean score was $3.58(\mathrm{SD}=0.91)$, in the second $3.63(\mathrm{SD}=0.91)$, and in the third $3.72(\mathrm{SD}=0.92)$. Table 4.25 shows that self-assessments have a high correlation with operational Internet skills. However, correlations with formal, information, and Strategic Internet skills appear to be medium to low. Apparently, the subjects have primarily operational skills in mind when assessing their general level of skills, again showing that these self-assessments provide an overly flattering picture.

Table 4.25 Pearson Correlations between Internet Skills Self-assessments and the Number of Successfully Completed Operational, Formal, Information, and Strategic Internet Skills Tasks

\begin{tabular}{lcccc}
\hline & $\begin{array}{c}\text { Operational } \\
\text { skills }\end{array}$ & $\begin{array}{c}\text { Formal } \\
\text { skills }\end{array}$ & $\begin{array}{c}\text { Information } \\
\text { skills }\end{array}$ & $\begin{array}{c}\text { Strategic } \\
\text { skills }\end{array}$ \\
\hline Self-assessment Study 1 & $.54^{\star \star}$ & $.40^{\star \star}$ & $.28^{\star \star}$ & $.31^{\star \star}$ \\
Self-assessment Study 2 & $.56^{\star *}$ & $.32^{\star *}$ & $.37^{\star *}$ & $.31^{\star *}$ \\
Self-assessment Study 3 & $.63^{\star \star}$ & $.69^{\star \star}$ & $.29^{\star \star}$ & $.29^{\star \star}$ \\
\hline
\end{tabular}

${ }^{\star *} p<.01$

Self-assessments do not provide a complete and valid measurement of Internet skills. Furthermore, they also tend to draw inaccurate conclusions. Men rated themselves significantly higher than women in studies 1 and 2 (study 1: $F(1,108)=4.55, p<.01$; study $2: F(1,108)=5.52, p<.05$ ) (see Table 4.26). However, in the actual performance test, no significant difference between males and females was observed. Post Hoc tests (Bonferroni with a 5\% significance level) among four categories of age reveal that subjects in the youngest age group(s) rate themselves significantly higher than subjects in the oldest age group(s) (study 1: $F(3,105)=10.50, p<.001$; study $2: F(3,105)=9.08, p<.001$; study $3: F(3,77)=11.71$, $p<.001$ ) (see Table 4.30). This confirms that the subjects have mainly operational skills in mind when assessing their own level of Internet skills. These are the areas in which subjects in the youngest group performed best. It is safe to say that the performance test is a more valid measurement than the self-assessment.

Table 4.26 Internet Skills Self-assessment (5-point scale) over Gender and Age (M(SD))

\begin{tabular}{lllllll}
\hline \multicolumn{6}{c}{ Gender } & \multicolumn{4}{c}{ Age } \\
& \multicolumn{1}{c}{ Male } & \multicolumn{1}{c}{ Female } & \multicolumn{1}{c}{$18-29$} & \multicolumn{1}{c}{$30-39$} & \multicolumn{1}{c}{$40-54$} & \multicolumn{1}{c}{$55-80$} \\
\hline Study 1 & $3.80(0.75)_{\mathrm{a}}$ & $3.38(0.99)_{\mathrm{b}}$ & $4.20(0.58)_{\mathrm{a}}$ & $3.78(0.80)_{\mathrm{a}, \mathrm{b}}$ & $3.41(0.75)_{\mathrm{b}, \mathrm{c}}$ & $3.03(1.00)_{\mathrm{c}}$ \\
Study 2 & $3.82(0.85)_{\mathrm{a}}$ & $3.42(0.94)_{\mathrm{b}}$ & $4.30(0.72)_{\mathrm{a}}$ & $3.70(0.88)_{\mathrm{a}, \mathrm{b}}$ & $3.34(0.94)_{\mathrm{b}}$ & $3.27(0.74)_{\mathrm{b}}$ \\
Study 3 & $3.80(0.80)_{\mathrm{a}}$ & $3.48(0.95)_{\mathrm{a}}$ & $4.08(0.72)_{\mathrm{a}}$ & $4.31(0.63)_{\mathrm{a}}$ & $3.57(0.93)_{\mathrm{a}, \mathrm{b}}$ & $2.96(0.77)_{\mathrm{b}}$ \\
\hline
\end{tabular}

For gender and age, within each row, means with non-common subscripts are significantly different, $p<.05$. 
Finally, criterion related evidence assumes external yardsticks in one way or another. Since such a yardstick does not exist for Internet skills, only secondary types of evidence can be looked for. A case of (predictive) criterion validity would be a correlation between the results of the performance test or framework and amount and variation of Internet use (more Internet skills are expected to lead to more Internet use and to more advanced types of Internet use). A case of (concurrent) criterion validity would be the concurrent results of more tests of the same framework after each other. This is what is attempted in this study. When an increasing number of the same test leads to the same results among the same population of test subjects, a commonly accepted criterion can be built after some time.

\subsection{Conclusions}

Research Question 4 addressed the Internet skills level of the Dutch population. From the results of three conducted performance tests, it is tempting to conclude that the Dutch population possesses a fairly high level of operational and formal Internet skills and that the levels of information and strategic Internet skills attained are much lower. However, it is not possible to draw conclusions about absolute levels of performance. There are few other performance tests of users' Internet skills (e.g., Hargittai, 2002), but these did not distinguish between the four levels of Internet skills, which leaves us without direct standards of comparison in other countries. In Chapter 7 , the results are put in a larger perspective and five general claims are made about the performance test results. An important conclusion is that operational and formal Internet skills are a necessary but not sufficient condition for the performance of information skills and strategic Internet skills when using the Internet. Therefore, policy makers and new media developers should adjust their beliefs that, with the exception of some seniors, now everybody has access to and can use the Internet.

Research Question 5 addressed the factors that determine Internet skills levels. From the results of the three studies, it appears that the level of Internet skills proved quite different among categories of the Dutch population. Educational level attained appeared the most important contributing factor, significant to the number of tasks completed successfully on all four types of Internet skills and, in most cases, also to the amount of time spent on tasks in all three studies. 
Furthermore, besides a direct contribution from educational level of attainment on both the medium-related skills and the content-related skills, there is also an indirect contribution, since the level of medium-related skills also in large part contributes to the level of the content-related skills. Age is the second most important contributing factor. It seems that age contributes negatively to the level of operational and formal Internet skills. However, there is a direct positive relation to the information and strategic Internet skills. However, the absolute result is that older age groups do not perform better on these content-related skills because these groups are hindered by their low levels of operational and formal Internet skills. Internet experience only contributes to the number of operational tasks completed and time spent on them. Amount of time spent online only showed some minor contribution to the time spent on formal Internet tasks. It seems that information and strategic Internet skills do not grow with years of Internet experience or the number of hours spent online. Taking an Internet course, having a support network, location of Internet use, and working condition all have only a minor influence on all four Internet skills.

The main benefits of the proposed Internet skills definition are as follows: (1) a definition and measurement of several types of Internet skills distinguished by others in the literature, (2) a definition representing a full range of Internet skills, and (3) the idea of a sequential and conditional nature of medium-related skills and content-related skills. Research Question 7 addressed the reliability and the validity of the Internet skills definition as a particular type of scientific measurement. In this chapter, the reliability of the studies flows from the mutually supportive conclusions of all three studies. The construct-related evidence of validity is confirmed by the empirical support for the theoretical definition, which suggested that the proposed definition consisted of a medium-related skills part (operational and formal skills) and a content-related skills part (information and strategic skills) with a particular coherence that is expected to have a conditional nature. The convergent validity appeared sufficient, since modest correlations between Internet skills and other constructs or measures of Internet skills were visible. 

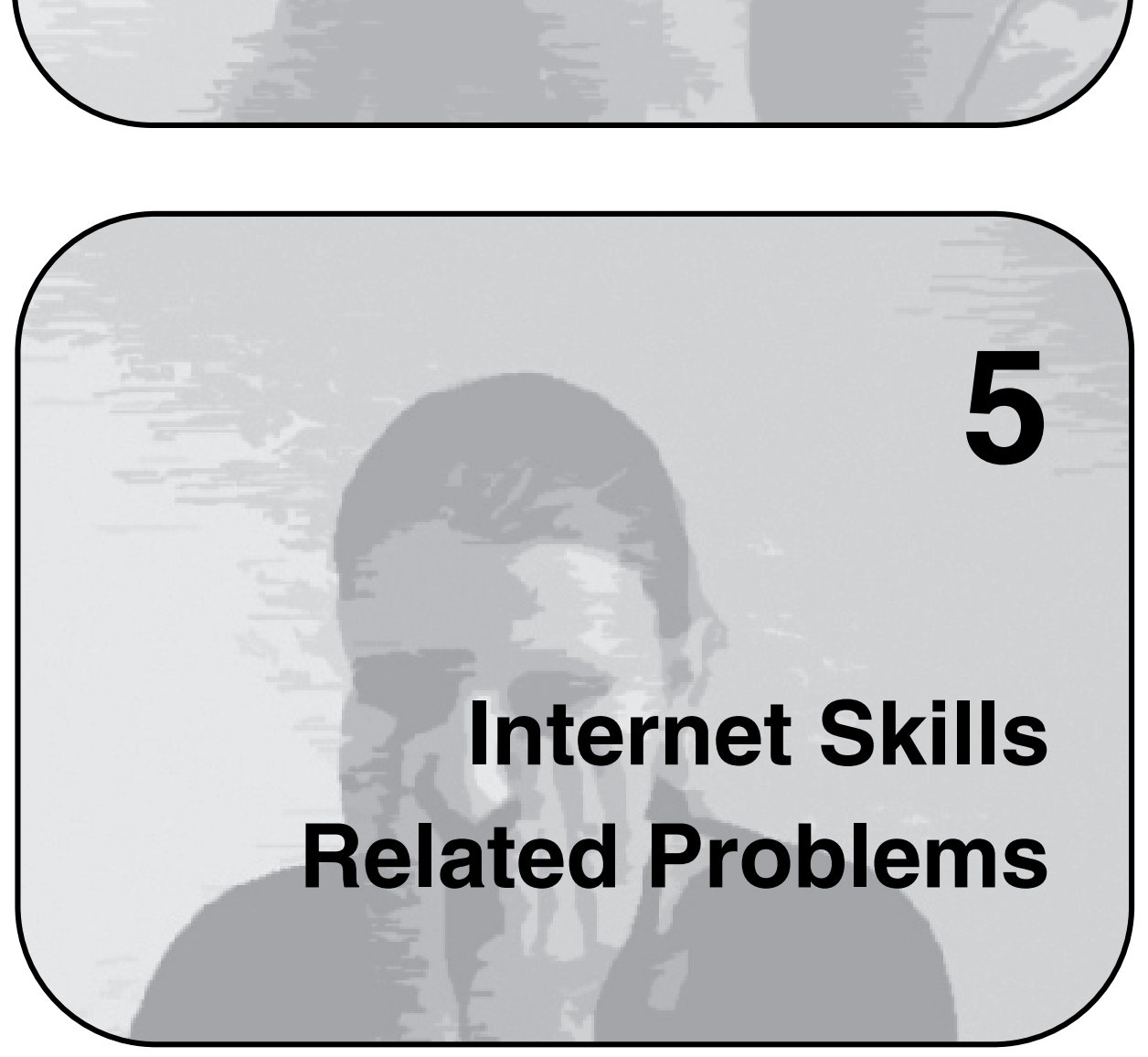

In Chapter 4, mainly absolute levels of Internet skills and differences in Internet skills among specific user groups were reported. It remained unclear why some people possess high levels of a specific Internet skill, while others barely master the same skills. This chapter provides a qualitative analysis of specific individual skill-related problems that users experience online. After discussing the applied coding scheme, the method and results are discussed. Finally, conclusions based on the qualitative analyses are presented. 


\subsection{Introduction}

Male subject-age 73-conducting a search operation that does not return any results: "Hmmm, let's wait for a while; the computer is still thinking." [Sits back and waits for seven minutes]

Female subject-age 19—conducting a search operation that does not return any results in the next two seconds: "****, nothing; let's try it elsewhere." LVisits another Web site while search engine is still processing the search query]

From the two situations above, we can learn that different people have different ways of working with the Internet. In these examples, both the young female and male senior unwittingly experienced a problem. The senior subject was under the impression that the search engine was still searching for results and kept on waiting in vain, while the younger subject was so inpatient that she missed out on relevant search results that did not get the chance to show up. This chapter provides a qualitative analysis in which several individual Internet skill-related problems are identified. The conclusion provides an answer to Research Questions 7 (Which specific individual skills-related problems do users experience online?) and 8 (Which factors determine the type and amount of Internet skills-related problems experienced?).

To answer the research questions, the screen actions of all 109 subjects that participated in the first study are analyzed. Morae recorder software (TechSmith, Version 2.2) was used to record the screen actions of the subjects that completed the assignments. Detailed log files of the recordings were created with Morae Manager (TechSmith, Version 2.2) to enable in-depth analysis. In total, 109 sets of data were generated by the screen recordings analyzed. The richness of the video data required a systematic approach for coding. The coding scheme used is discussed in the next section. Section 5.3 presents the results of the analysis, followed by an overall conclusion and points of discussion.

\subsection{Coding Internet skills-related problems}

To analyze the video recordings, a coding scheme was developed based upon both the Internet skills definitions discussed in Chapter 3 and trends observed in the 
data. Most of the operational and formal Internet skills-related problems in the coding scheme emerged from the matching skill definitions. A specific task was created to reveal whether subjects would experience them. During the completion of the information and strategic skills assignments, the occurrences of specific experienced information and strategic skills-related problems were counted. Although most of the problems emerged from the conceptual Internet skills definition, some problems were derived from the data. Take, for example, the following part of the information Internet skills definition: selecting information. Analysis of the data revealed that there are more problems regarding this part alone (for example, not going beyond the first search result, choosing sponsored search results without knowing, or choosing irrelevant search results). The same accounts for the strategic skills definition (for example, analysis of the data revealed that taking actions towards a final goal contained the problems of being misled, using information from only one source when more were needed, working in an unstructured manner, and using Web sites that support decisions incorrectly). In short, problems that emerged from the data and were not directly mentioned in the Internet skills definitions were added to the coding scheme. In total, 38 problems were identified, described at a general level of abstraction (instead of a concrete level such as mouse clicks or keystrokes) (see Table 5.1).

To test the reliability of the empirical work, the coding of the data was replicated by a second observer. This observer independently coded the recordings of ten subjects, which was compared to the coding of the primary observer. The comparison revealed no disagreements on the coded operational and formal skillrelated problems (with the binary outcome experienced/not experienced in the specific task). The number of the individual information skill-related problems that were counted during the completion of the information skill-related assignments did not reveal any points for discussion. However, some disagreement between coders on three strategic skill problems occurred, which was solved after discussion. One of the points of discussion, for example, was whether filling out a form irrelevant to the assignment itself could also be coded as making an incorrect decision. Each session of video recording took about two to three hours to code and analyze. 
Table 5.1 Coding Scheme

\begin{tabular}{|c|c|}
\hline \multicolumn{2}{|c|}{ Individual Operational Internet Skills Problems } \\
\hline Address bara,b & Using the address bar incorrectly (e.g., entering keywords) \\
\hline Save & Not being able to save a file to the hard disk \\
\hline PDFa & Not being able to save a PDF file \\
\hline Favorites & Not being able to add a Web site to the favorites (or bookmarks) \\
\hline Form ${ }^{a, b}$ & Using a Web form incorrectly (e.g., buttons or pull-down menus) \\
\hline Search engine ${ }^{a, b}$ & Not recognizing the search engine or input field \\
\hline Search queries ${ }^{a, b}$ & Using search queries incorrectly \\
\hline Mouse $\mathrm{b}^{\mathrm{b}}$ & Using the mouse incorrectly (e.g., double click or right click) \\
\hline Scrollbarb & Experiencing scrollbar related problems \\
\hline \multicolumn{2}{|c|}{ Individual Formal Internet Skills Problems } \\
\hline Design_Web site & Experiencing problems with different Web site designs \\
\hline Design_Menua,b & $\begin{array}{l}\text { Using Web site menus incorrectly (e.g., not being able to use } \\
\text { scroll-over menus) }\end{array}$ \\
\hline Orientation_Within ${ }^{\mathrm{a}, \mathrm{b}}$ & Not knowing where one is located within a Web site \\
\hline Orientation_Between & Entering automatically opened browser windows without realizing this \\
\hline Orientation_Search ${ }^{\mathrm{a}, \mathrm{b}}$ & Not being able to open more than one search result \\
\hline \multicolumn{2}{|c|}{ Individual Information Internet Skills Problems } \\
\hline System_Proper & Not choosing a proper search system or way of searching \\
\hline Queries_Wrong & Using too broad search queries not emergent from the search task \\
\hline Queries_Specific & Using search queries specific to the task \\
\hline Queries_Booleans & Not using Booleans to limit search results (e.g., parentheses) \\
\hline Search_Advanced & Not using advanced search methods (e.g., date or excluding keywords) \\
\hline Search_Limit & Not searching within search results \\
\hline Search_Luck & Using the Google option "I'm feeling lucky" \\
\hline Select_Sponsor & Choosing sponsored or commercial results \\
\hline Select_First three & Not checking more than the first three search results \\
\hline Select_First page & Not checking more than the first page of search results \\
\hline Select_Irrelevant & Choosing irrelevant search results \\
\hline Information_Form & Filling out a form that does not lead to the necessary information \\
\hline Information_Wrong & Using information that is not applicable to the situation \\
\hline Information_Source & Using information from a less reliable Web site \\
\hline Information_Date & Using information that is outdated \\
\hline Information_Check & Not checking information on another Web site \\
\hline \multicolumn{2}{|c|}{ Individual Strategic Internet Skills Problems } \\
\hline Orientation_Stimuli & Being distracted by irrelevant stimuli (e.g., banners) \\
\hline Orientation_Start & Not knowing how or where to start with the assignment \\
\hline Action_Misled & $\begin{array}{l}\text { Being misled (e.g., working towards a goal that does not deliver } \\
\text { personal benefits) }\end{array}$ \\
\hline Action_Source_Single & Using information from only one Web site (source) \\
\hline Action_No_Structure & Working in a unstructured (random) way towards the final answer \\
\hline Action_Support_Wrong & Using Web sites incorrectly that support the decision-making process \\
\hline Decision_Wrong & Making an incorrect decision based on the information acquired \\
\hline Decision_No & Not making a decision at all \\
\hline Decision_Incomplete & Making a decision based on incomplete information \\
\hline
\end{tabular}




\subsection{Identifying Internet skills-related problems}

The next sections discuss the individual Internet skills-related problems that emerged from the Internet skills definition and the completion of the assignments in the first performance test. During the performance of the information skills assignments, both the operational and formal skill problems are recorded during specific tasks designed to measure the corresponding skills. To measure the experienced information and strategic skills problems, the total numbers of occurrences of every problem are counted in all 109 screen recordings so as to make statistical analysis possible.

\subsubsection{Operational Internet skills-related problems}

For 5\% of all 109 subjects, the use of the address bar appeared to be problematic. Three seniors did not recognize the address bar at all. Without the immediate appearance of Google, they were unable to recognize the Internet browser and had no clue on how to start the session. One 55-year-old medium-educated man did not remove the "about: blank" part in the address bar. A 44-year-old loweducated woman was convinced that she could only open a Web site using the "Open" option in the File-menu of Internet Explorer.

Saving a file to the hard disk (in this case, the online tax declaration program) was problematic for $37 \%$ of the subjects. The largest percentage of the subjects who failed this test saved the whole Web page $(12 \%)$ or had absolutely no clue on how to proceed (11\%). Other mistakes included assuming that the file was already saved after opening the save dialog only, making Web site shortcuts to the desktop, or adding a Web site to the favorites.

In one task, subjects were asked to open an online PDF file and save it in an existing folder on the desktop. Forty-nine percent of the subjects experienced problems and did not succeed. Twenty-five percent had absolutely no idea what to do, and $15 \%$ were only able to save the PDF in the automatically opened "My documents" folder. Others added the file to the "Favorites," closed the save dialog instead of saving the file, right-clicked on the opened PDF file, or saved the whole Web site before opening the PDF itself. 
Ninety percent of the subjects were able to add a Web site to the Favorites (or Bookmarks in Mozilla Firefox).

For only four subjects, filling out a Web-based form appeared to be a problem. They forgot to complete the whole form, which resulted in a warning message that caused confusion. During the information assignments, five subjects experienced this problem.

Problems with respect to the operation of search engines were rare. Four subjects overlooked the search engine on a Web site (while obviously present), and 11 subjects $(10 \%)$ experienced search query-related problems. These were typed without spaces or preceded by "www." Ten senior subjects (33\% of all seniors) entered search queries into the address bar.

Finally, three subjects in the oldest age group (10\% of all seniors) only used the scrollbar's tiny arrow buttons for traversing long distances on a page. They were not aware of the easier-to-use middle part of the scrollbar. Using the mouse resulted in some surmountable problems for 14 seniors (47\% of all seniors), causing minor delays. These subjects clicked multiple times on search buttons or used the right button instead of the left.

Failing to save a file to the hard disk, to open and save an online PDF file, and to add a Web site to the Favorites and operational problems with respect to the use of search engines appeared most frequently, and these problems are accounted for in regression analyses summarized in Table 5.2. The analysis is conducted to provide details about the different users that experience these problems when completing the assignments. The specific factors accounted for in the linear regression analyses reported earlier in this chapter are added to the analysis. Since the experienced problems are measured in a specific task with a binary outcome (not succeeded/succeeded), logistic regression analyses are performed to identify what factors appear significant in the regression model. In the models for saving a file and opening and saving a PDF, age is a negative $(\operatorname{Exp}(B)<1)$ contributor. Education has a positive $(\operatorname{Exp}(\mathrm{B})>1)$ contribution to saving a PDF, adding a Web site to the Favorites, and using a search engine. Other factors did not appear significant. 
Table 5.2 Logistic Regression Analysis of Operational Skills-related Problems

\begin{tabular}{|c|c|c|c|c|}
\hline Independent variables & $\begin{array}{l}\text { Saving a } \\
\text { file } \\
\text { Exp(B) }\end{array}$ & $\begin{array}{c}\text { Opening and } \\
\text { saving a PDF } \\
\operatorname{Exp}(B)\end{array}$ & $\begin{array}{c}\text { Adding sites to } \\
\text { the Favorites } \\
\operatorname{Exp}(B)\end{array}$ & $\begin{array}{c}\text { Using search } \\
\text { engines } \\
\operatorname{Exp}(B)\end{array}$ \\
\hline Gender & 1.21 & 0.33 & 0.81 & 1.05 \\
\hline Age & $0.95^{\star}$ & $0.91^{* \star *}$ & 0.95 & 0.95 \\
\hline Education & 1.72 & $5.59^{* \star *}$ & $3.17^{*}$ & $3.67^{\star}$ \\
\hline Years online & 1.23 & 1.33 & 0.97 & 1.48 \\
\hline Hours online weekly & 1.00 & 1.01 & 1.04 & 1.09 \\
\hline Internet class (no / yes) & 1.15 & 0.76 & 0.69 & 0.34 \\
\hline Assistance required (no / yes) & 0.54 & 0.94 & 0.30 & 0.68 \\
\hline $\begin{array}{l}\text { Primary location of Internet use (at } \\
\text { home / elsewhere) }\end{array}$ & 4.44 & 1.29 & 1.62 & 3.08 \\
\hline Working situation (inactive / active) & 3.12 & 1.03 & 1.55 & 2.16 \\
\hline Nagelkerke $R^{2}$ & .47 & .61 & .48 & .64 \\
\hline Chi-square & $45.84^{\star * \star}$ & $65.64^{* * *}$ & $41.58^{\star \star \star}$ & $66.66^{* * *}$ \\
\hline
\end{tabular}

\subsubsection{Formal Internet skills-related problems}

To identify individual layout and Web site design related problems, subjects were asked to find the street address (simple information) of three public agencies (very different in Web site design) in the Dutch city Nijmegen. Twenty-one percent did not succeed. Some subjects simply overlooked the "contact" button in the main menu, while others did not recognize this menu at all. There was one subject who altered the URL by adding "/Nijmegen," believing that this would bring him directly to the contact details. During the information tasks, $40 \%$ of the subjects experienced problems while using (rollover) menus.

Maintaining orientation within a Web site was difficult for $28 \%$ of the subjects. In a specific task, subjects were asked to return to the homepage of the Dutch Tax and Customs administration from a deep-link. One of them tried to find the homepage using the Internet browser's help function, and another attempted to call his brother to ask him for help finding the homepage. During completion of the information assignments, subjects often believed that they were on the homepage of a Web site while this was not the case; they clicked the link "Up" while they were already at the top of a page or clicked a link to the current page.

To measure problems related to orientation between Web sites, subjects were instructed to click an external link on the Web site of the Government Information Service (Central Office of Information). Twenty-one percent of the 
subjects lost their orientation when a new browser window was opened. They did not understand why the back button was deactivated and overlooked the Government Information Service's Web site in the original window, even when it was still visible in the background. Some of the participants closed all windows and started again. During the completion of the information assignments, 29\% of the subjects experienced similar problems. They were relocated without noticing and did not see the original window anymore after opening a new one.

Maintenance of orientation when navigating search results was measured by asking subjects to open the first and fourth search result after performing a search operation. One third of subjects experienced problems, the main one being that after opening the first search result, subjects chose the fourth option in another nearby menu that had nothing to do with the generated search results. They were convinced that they opened the fourth search result, indicating that the Web site's structure caused confusion. Two subjects opened the fourth page with search results instead of the fourth search result. During completion of the information assignments, ten subjects never returned to the original search result list. They retyped the same search queries in a new Google window.

Table 5.3 Logistic Regression Analysis for Formal Skills-related Problems

\begin{tabular}{lcccc}
\hline Independent variables & $\begin{array}{c}\text { Using } \\
\text { different Web } \\
\text { site designs } \\
\text { Exp(B) }\end{array}$ & $\begin{array}{c}\text { Orientation } \\
\text { within a Web } \\
\text { site } \\
\text { Exp(B) }\end{array}$ & $\begin{array}{c}\text { New } \\
\text { browser } \\
\text { window } \\
\text { Exp(B) }\end{array}$ & $\begin{array}{c}\text { Browsing } \\
\text { more search } \\
\text { results } \\
\text { Exp(B) }\end{array}$ \\
\hline Gender & 1.06 & 1.02 & 1.64 & 0.90 \\
Age & $0.93^{*}$ & $0.95^{*}$ & $0.97^{\star}$ & 0.97 \\
Education & 1.71 & 1.11 & $2.72^{* * *}$ & 1.47 \\
Years online & 1.03 & 1.02 & 0.95 & $1.48^{*}$ \\
Hours online weekly & 0.98 & 0.88 & 1.01 & 1.00 \\
Internet class (no / yes) & 0.82 & 0.56 & 0.56 & 1.00 \\
Assistance required (no / yes) & $0.06^{*}$ & 0.06 & 0.49 & 0.37 \\
Primary location of Internet use (at & 2.57 & 1.54 & 1.25 & 6.73 \\
home / elsewhere) & 0.98 & 0.92 & 2.20 & 0.97 \\
Working situation (inactive / active) & .87 & .59 & .39 & .44 \\
\hline Nagelkerke $R^{2}$ & $40.18^{* * *}$ & $41.64^{* * *}$ & $37.33^{* * *}$ & $40.73^{* * *}$ \\
Chi-square & & & &
\end{tabular}

According to Table 5.3 (in which the binary outcome is "succeeded/ not succeeded"), in the regression models for using different Web site designs, maintaining orientation within a Web site, and getting confused when a new browser window is opened, age is a negative contributor. Like the younger 
subjects, the higher educated are less likely to experience the problem of getting confused when new browser windows are opened. Furthermore, the more experienced Internet users are, the more likely they are to be able to open more than one search result. Finally, subjects that need help from peers when using the Internet experience more problems with using different Web site designs.

\subsubsection{Information Internet skills-related problems}

Three assignments were prepared to identify information Internet skill-related problems. The first assignment used a closed environment (one specific Web site). In the second and third assignments, subjects could choose their own starting point. All subjects, with the exception of two, chose Google.

Defining search queries is a step that revealed large differences between the subjects. Fifty-six percent of the subjects performed search operations using search queries that did not fit the information problem or were too general (e.g., keyword "salary" when searching for "minimum wages in a specific year"). In a few cases, the full assignment was entered into the search bar, resulting in unusable search results.

Ninety-five percent of the subjects did not refine the search results by using Boolean operators. In only four cases, quotation marks were used, and in two cases, the "+" symbol was used. Nobody used advanced search methods (e.g., entering exact word combinations or dates).

Since search engines return a vast number of unsuitable search results, intensive selection is required before the results become useful. Two subjects selected the Google option "I'm feeling lucky." Sponsored or commercial results were opened by $56 \%$. This percentage is probably higher than in other contexts since the Dutch government usually appears at the top of the search result lists in Google with sponsored links. Thirty-six percent of the participants did not go beyond the first three search results $(12 \%$ went beyond the first three results in all information assignments). Ninety-one percent did not go further than the first page with search results in all the assignments. Fifty-five percent of the participants selected one or more irrelevant search results, $20 \%$ selected irrelevant information pages within Web sites, and 4\% filled out irrelevant forms in an attempt to find the 
information needed (e.g., filling in forms that generate average wages for specific jobs instead of searching for the minimum wages as assigned).

Six percent of the subjects used information from an unreliable Web site. A striking observation was that nobody seemed to pay attention to the source of the information found. Finding the answer seems to be the primary objective; it does not seem to matter where the information comes from. For example, government information was taken from a Web site containing classroom talks of primary school pupils. In addition, nobody evaluated the date the information was published. Only four subjects checked information on another Web site.

Table 5.4 Linear Regression Analysis for Formal Skills-related Problems

\begin{tabular}{|c|c|c|c|c|c|c|c|c|}
\hline \multirow[t]{2}{*}{ Independent variables } & \multicolumn{2}{|c|}{$\begin{array}{l}\text { Using too broad } \\
\text { search queries }\end{array}$} & \multicolumn{2}{|c|}{$\begin{array}{l}\text { Limited use of } \\
\text { search results }\end{array}$} & \multicolumn{2}{|c|}{$\begin{array}{c}\text { Irrelevant } \\
\text { search results }\end{array}$} & \multicolumn{2}{|c|}{$\begin{array}{l}\text { Using irrelevant } \\
\text { information }\end{array}$} \\
\hline & $t$ & $\beta$ & $t$ & $\beta$ & $t$ & $\beta$ & $t$ & $\beta$ \\
\hline Gender (M/F) & 0.57 & .05 & 0.53 & .12 & -0.14 & -.01 & -1.06 & -.10 \\
\hline Age & -0.38 & -.05 & -0.43 & -.12 & -2.30 & $-.32^{\star}$ & -0.62 & -.08 \\
\hline Education (low-medium-high) & -2.48 & $-.27^{\star *}$ & -1.81 & $-.31^{*}$ & -1.41 & -.17 & -2.25 & $-.26^{*}$ \\
\hline Years online & -1.37 & -.14 & -1.70 & -.45 & -0.86 & -.10 & -0.19 & -.02 \\
\hline Hours online weekly & -0.92 & -.10 & -0.76 & -.01 & -0.65 & -.07 & 0.65 & .07 \\
\hline Internet class (no / yes) & 0.85 & $.08^{*}$ & 0.76 & .03 & -0.41 & -.04 & -0.58 & -.06 \\
\hline Assistance required (no / yes) & 0.79 & .08 & 0.79 & .05 & 0.52 & .06 & 1.45 & .16 \\
\hline $\begin{array}{l}\text { Primary location of Internet } \\
\text { use (at home / elsewhere) }\end{array}$ & 0.14 & .01 & 0.13 & .03 & 0.63 & .07 & -0.67 & -.07 \\
\hline $\begin{array}{l}\text { Working situation (inactive / } \\
\text { active) }\end{array}$ & -1.73 & .20 & -0.98 & .48 & -0.73 & -.09 & -0.63 & -.08 \\
\hline$R^{2}$ & & .26 & & .21 & & .22 & & .15 \\
\hline$F$ & & $3.85^{\star \star \star}$ & & $2.76^{\star \star \star}$ & & $2.84^{\star \star \star}$ & & $1.97^{*}$ \\
\hline
\end{tabular}

During the completion of the information skill assignments, the total number of problem occurrences was counted. Linear regression analyses over this number that revealed significant $F$-values are reported in Table 5.4. In the model for selecting irrelevant search results, age is predictive. Surprisingly, the older the subjects are, the less likely they are to select irrelevant search results. Education is predictive for using broad search queries, not going beyond the first three search results, and selecting irrelevant information, where the higher educated experience these problems less than the lower. Other factors do not appear predictive to the regression models. 


\subsubsection{Strategic Internet skills-related problems}

Maintaining orientation towards the final goal was difficult for some subjects. Strategic skill-related problems that emerged in this step were being distracted by irrelevant stimuli (e.g., banners) (4\%), not having a clue on how to start at least one of the two strategic assignments (49\%), and being misled during the completion of the assignments without noticing (20\%).

A few strategic Internet skills-related problems emerged from the second step in the definition: taking action toward the final goal. Thirty-six percent of the subjects used Web sites that support users in making informed decisions (e.g., choosing a political party). Unfortunately, these Web sites were only used in the simplest way, and the generated outcomes were too easily taken for granted. Of the subjects that used these Web sites, $92 \%$ were not able to generate useful outcomes. Twenty-five percent of the subjects did not combine multiple information sources and used information from one Web site only, not enough to resolve the strategic assignment (e.g., making decisions taking into account different political views). Seventy-one percent of the subjects experienced the problem of working in an unstructured way (determined by checking whether information is not gathered piece by piece but by randomly surfing) in either of the two strategic assignments.

In the final stage of the strategic skill definition, an important problem appeared to be making misguided decisions based on the information found. This was done by $46 \%$ of subjects. Sixty-three percent based their decisions on incomplete information tenure.

Linear regression analyses over the total occurrences of specific problems that revealed significant F-values are reported in Table 5.5. In the models for not knowing how or where to start, using only one Web site to make decisions, working in an unstructured manner, and making incorrect decisions based on the information found, education is predictive. Subjects with more Internet experience have fewer problems knowing how to start an assignment. Surprisingly, subjects that followed an Internet course are less likely to know how to start a strategic skill assignment. They do however make fewer incorrect decisions based on the information found. 
Table 5.5 Linear Regression Analysis of Strategic Skills-related Problems

\begin{tabular}{|c|c|c|c|c|c|c|c|c|}
\hline \multirow[t]{2}{*}{ Independent variables } & \multicolumn{2}{|c|}{$\begin{array}{l}\text { Not knowing } \\
\text { where to Start }\end{array}$} & \multicolumn{2}{|c|}{$\begin{array}{l}\text { Using only one } \\
\text { Web site }\end{array}$} & \multicolumn{2}{|c|}{$\begin{array}{c}\text { Working } \\
\text { unstructured }\end{array}$} & \multicolumn{2}{|c|}{$\begin{array}{l}\text { Making incorrect } \\
\text { decisions }\end{array}$} \\
\hline & $t$ & $\beta$ & $t$ & $\beta$ & $t$ & $\beta$ & $t$ & $\beta$ \\
\hline Gender (M/F) & 0.83 & .07 & 0.49 & .05 & -0.15 & -.01 & -1.18 & -.11 \\
\hline Age & 0.34 & .04 & -0.94 & -.12 & 0.91 & .11 & 0.69 & .09 \\
\hline Education (low-medium-high) & -2.48 & $-.26^{* *}$ & 2.45 & $.27^{\star}$ & -3.10 & $-.34^{* *}$ & -2.81 & $-.32^{\star \star}$ \\
\hline Years online & -2.84 & $-.29^{\star \star}$ & -0.41 & -.04 & 0.29 & .03 & 0.17 & .02 \\
\hline Hours online weekly & -0.27 & -.03 & -1.40 & -.15 & -0.15 & -.02 & -0.32 & -.04 \\
\hline Internet class (no / yes) & 2.19 & $.19^{*}$ & 1.35 & .12 & 0.26 & .02 & -2.07 & $-.19^{\star}$ \\
\hline Assistance required (no / yes) & 0.09 & .01 & -2.49 & $-.27^{*}$ & 0.13 & .01 & -0.64 & -.07 \\
\hline $\begin{array}{l}\text { Primary location of Internet } \\
\text { use (at home / elsewhere) }\end{array}$ & -0.10 & -.01 & 2.22 & .21 & -1.87 & -.18 & 2.37 & .23 \\
\hline $\begin{array}{l}\text { Working situation (inactive / } \\
\text { active) }\end{array}$ & -0.64 & -.07 & -0.76 & -.09 & -1.10 & -.13 & 0.74 & .09 \\
\hline$R^{2}$ & & .31 & & .23 & & .25 & & .18 \\
\hline$F$ & & $4.84^{* \star *}$ & & $3.37^{* * *}$ & & $3.62^{* \star *}$ & & $2.44^{*}$ \\
\hline
\end{tabular}

\subsection{Conclusions}

Research Questions 7 and 8 addressed specific individual skills-related problems that users experience when using the Internet and the factors that determine the type and amount of Internet skill problems experienced. The results from the analysis revealed that lower levels of educational attainment and higher age largely contribute to individual operational and formal skill-related problems, limiting basic Internet use.

- The major operational problems are saving files or PDF documents. Using search engines as instruments of support is also not a natural task for everyone (e.g., entering keywords in the address bar or typing keywords attached to each other). The mouse, scrollbar, and Web-based forms caused only some problems for subjects with low levels of educational attainment and seniors.

- Regarding individual formal skills-related problems, the most frequently experienced problem was the lack of orientation when navigating between Web sites, but also within Web sites and between search results. Moreover, Web sites' menus-especially rollover-were sources of confusion. Seniors and low-educated participants were the most likely to experience these problems. 
- A major information skill-related problem seems to be the formulation of unsuitable or overly general search queries. Lack of education is a strong contributor to this problem. Another problem is the lack of knowledge about employing Boolean operators to refine searches. The use of Boolean operators is not generally known, and the interface to compose queries with them is hidden from initial view (Marchionini \& White, 2007). A problem that almost all subjects experienced is the limited use of most search results; typically, the first three results attracted attention, and the second page with results was only opened sporadically. This is in accordance with information-seeking studies (e.g., Aula \& Nordhausen, 2005; Birru et al., 2004). The results also revealed that the older the subject, the less relevant search results were selected. The fact that few users tended to evaluate the information found represents an important problem.

- Regarding strategic skill-related problems, it was revealed that the use of Web sites that support users in making informed decisions (e.g., voting) only caused more problems. These Web sites are employed in the wrong way. Lower levels of educational attainment contributed to working in an unstructured manner. Another important problem was the fact that subjects had trouble keeping their focus on the original goal of the assignment. Although the number of distracted subjects was low (this number might be higher in real life since the test environment more or less forced the subjects to focus on the assignment), many of them did lose track of their personal goals.

The results of the analyses reveal that age is mainly related to operational and formal Internet skill-related problems. Though the number of operational and formal individual Internet skill-related problems is highest for the oldest age group, surprisingly, they do not experience more individual information and strategic skill-related problems. As for selection in search results, they scored even better than the youngest group. This underlines the importance of accounting for skill-related problems in the detailed way we have attempted here, and it shows the importance of paying attention to information and strategic skills in addition to operational and formal skills in educational and occupational settings. 

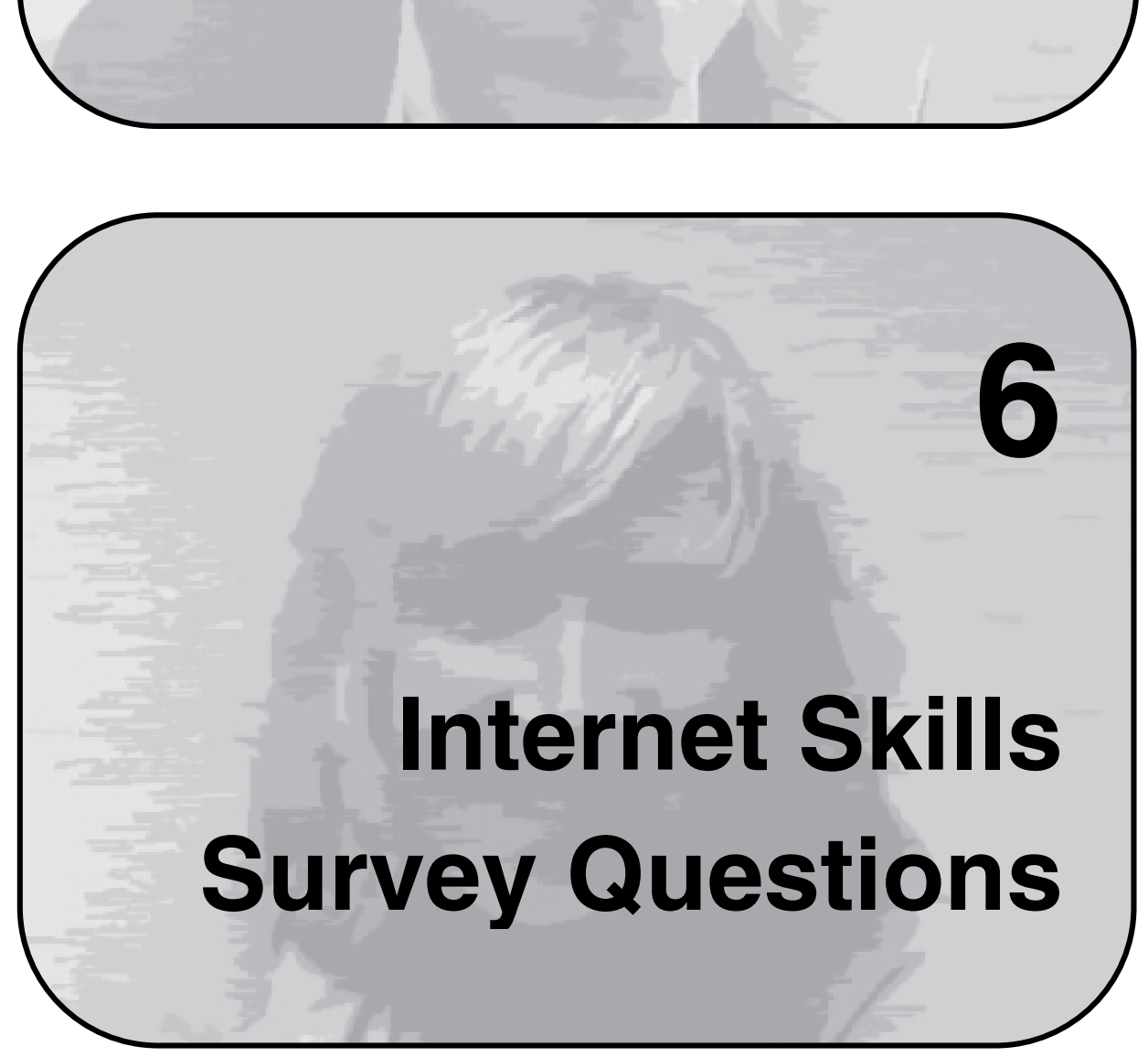

In Chapter 4, three performance tests were discussed in which the actual level of Internet skills was measured. Although these tests proved effective and valid, in the long term, they are too expensive and time-consuming for large-scale data collection. Therefore, in this chapter, a valid method of measuring Internet skills in surveys is sought. To date, surveys that attempt to measure Internet skills often use overly flattering self-assessments or indirect measures of skills. 


\subsection{Introduction}

Both nationally and internationally, policy makers are increasingly attempting to measure Internet skill levels among populations. In Europe, for example, both the databank Eurostat and the Flash Eurobarometer have been emphasizing the importance of Internet skills. Both benchmarks use surveys in which respondents are asked which of a number of activities they have ever carried out. There is no measurement or observation of actual Internet skills. Eurostat, for example, asks respondents which of the following Internet activities they have ever performed: using a search engine to find information, sending an email with an attachment, posting messages, making a telephone call via the Internet, sharing folders with other people so as to exchange media, and creating a Web page. Respondents are subsequently divided into a "low skills level" category of respondents that have carried out one or two of the activities, a "medium skills level" category of respondents that have carried out three or four of the activities, and a "high skills level" category of respondents that have carried out five or six of the activities. Internet skills are thus put on par with Internet usage, although the relation between the two variables is not clear.

Measuring Internet skills in large-scale performance tests is both expensive and time consuming. A useful addition to the measurement of Internet skills would be the development of survey questions for measuring Internet skills. In the United States, Hargittai (2005) proposed composite variables of survey items that served as better predictors of people's actual Internet skills based on performance tests than measures of users' self-perceived abilities, Internet experience, or amount of Internet use. She proposed survey measures that appear as useful additions to current measurements (or lack thereof). In this chapter, the idea of proposing survey items derived from the actual Internet skills levels is further investigated, this time by proposing and testing items for all four Internet skills. Research Question 9 (Which survey questions are valid and reliable measures of Internet skills?) is addressed by testing several items and measurement scales.

The structure of this chapter follows three steps that together result in survey measures for all four Internet skills. In the first step the correlations between several skills items and the actual performances (number of tasks completed successfully and the time spent) are measured for all four Internet skills. In the second step, the Fornell and Larcker (1981) discriminant validity criterion is used 
to test discriminant validity of the Internet skills items that showed the highest correlations in step one. Finally, in the third step the items are further analyzed using a first-order confirmatory factor analysis based on a new data sample.

\subsection{Step 1: Correlations between items and observed skills}

The most common response formats used in measurement scales are frequency (how often), evaluation (how much do you like it), and agreement (how much do you agree) (Redding, Maddock, \& Rossi, 2006). Frequency and agreement scales have been developed to measure Internet skills. The proposed items are derived from the Internet skills definition proposed in Chapter 3. The same subjects that participated in the second $(\mathrm{n}=109)$ and third $(\mathrm{n}=88)$ performance test were asked to complete a questionnaire after completing the assignments. This questionnaire presented Internet skills-related items that together took approximately eight minutes to complete. In the second study, the items used a frequency scale. Respondents were asked to complete items with response options that were anchored on a five-point Likert scale, ranging from Never (1) to Daily (5). The items measured the frequency of various Internet skill-related activities that are based on the Internet skills definition proposed in Chapter 3. Items for all four Internet skills were constructed and pretested among ten respondents for clearness, conciseness, understanding, and reading level. In the third study, the questionnaire used a seven-point Likert scale on which respondents were asked to rate their agreement with several Internet skills-related behaviors, ranging from Strongly disagree (1) to Strongly agree (7). In addition, here, the items for all four Internet skills were constructed and pilot-tested among ten respondents. In analyzing the responses from the questionnaire, all negatively keyed items are recoded. For missing values (13 in the second study and 10 in the third), mean substitution is applied; missing values are replaced by the average of the observed values for that item.

Table 6.1 shows the Pearson's correlation coefficients among the frequency scale items and the two outcomes of actual performance (i.e., percentage of tasks successfully completed and amount of time spent on the eight tasks). The signs of the coefficients are in the expected direction. In the majority of cases, the 
coefficients are statistically significant for both outcome skills measures. This suggests that the created items may be used as a proxy for actual skill measures.

Table 6.1 Pearson's Correlations between Items and the Number of Operational (O), Formal (F), Information (I), and Strategic (S) Internet Skills Assignments Completed Successfully and the Time Spent

\begin{tabular}{|c|c|c|}
\hline On the Internet, how often do you .... & $\begin{array}{c}\text { Successful Task } \\
\text { completion }\end{array}$ & Time spent \\
\hline save files & $.48^{* *}$ & $-.48^{\star \star}$ \\
\hline complete forms (e.g., application forms) & $.44^{\star \star}$ & $-.45^{\star \star}$ \\
\hline use the refresh button & $.44^{* *}$ & $-.45^{\star *}$ \\
\hline upload files to another computer & $.42^{\star *}$ & $-.44^{* *}$ \\
\hline use back and forward buttons & $.42^{\star \star}$ & $-.38^{\star \star}$ \\
\hline download programs from the Internet & $.41^{\star *}$ & $-.34^{\star \star}$ \\
\hline watch video files & $.46^{\star \star}$ & $-.51^{\star *}$ \\
\hline download music files & $.41^{* *}$ & $-.43^{\star \star}$ \\
\hline add a Web site to the "Favorites" & $.27^{\star \star}$ & $-.20^{*}$ \\
\hline save photos on your PC & $.26^{* *}$ & $-.19^{*}$ \\
\hline use multiple browser windows & $.56^{\star \star}$ & $-.66^{\star \star}$ \\
\hline find Web sites to be confusing & $-.37^{\star \star}$ & $.40^{\star *}$ \\
\hline get lost & $-.32^{\star *}$ & $.41^{\star *}$ \\
\hline feel disoriented & $-.29^{\star \star}$ & $.33^{\star *}$ \\
\hline experience difficulties with a Web site's layout & $-.29^{\star \star}$ & $.41^{* *}$ \\
\hline not know where you are & $-.28^{\star \star}$ & $.37^{\star *}$ \\
\hline check information retrieved on another Web site & $.44^{\star \star}$ & -.18 \\
\hline examine only the top results & $-.33^{\star *}$ & $.39^{\star \star}$ \\
\hline search for information & $.30^{\star \star}$ & $-.40^{\star \star}$ \\
\hline find the information you were looking for & $.30^{* *}$ & $-.26^{* *}$ \\
\hline use advanced search options (e.g., Boolean operators) & $.30^{* *}$ & $-.23^{*}$ \\
\hline examine the results on subsequent result pages & $.30^{\star *}$ & -.14 \\
\hline use more than one search keyword & $.24^{*}$ & $-.23^{*}$ \\
\hline evaluate the source of the information found & $.20^{\star}$ & -.18 \\
\hline make a decision based on retrieved information & $.48^{\star \star}$ & -.15 \\
\hline use information about a specific subject from multiple sites & $.42^{* *}$ & -.12 \\
\hline buy a product based on retrieved information & $.38^{* *}$ & -.10 \\
\hline benefit from using the Internet & $.31^{\star \star}$ & -.11 \\
\hline reach your intended goal & $.29^{\star *}$ & -.06 \\
\hline use reference Web sites & $.28^{* *}$ & -.04 \\
\hline gain financial benefits & $.27^{\star \star}$ & -.13 \\
\hline compare products & $.24^{*}$ & $-.21^{*}$ \\
\hline work towards a specific goal & $.22^{*}$ & $-.26^{*}$ \\
\hline
\end{tabular}

Table 6.2 shows the Pearson's correlation coefficients among the agreement scale items and the two items measuring actual performance. Here also, the signs of the coefficients are in the expected direction. However, the correlations, especially those concerning information and strategic Internet skills, are lower than the 
correlations between the frequency scale items and performance outcomes. In the majority of cases considering information Internet skills, the coefficients are not statistically significant for both outcome skills measures. This suggests that the created frequency items might be better suited for use as a proxy for actual skills measures. Therefore, the frequency scale items are further analyzed in steps two and three. More specifically, based on the correlations the following items are considered (and labeled to ease further analyses):

Operational Internet skills:

- O1 - save files

- $\mathrm{O} 2$ - complete forms

- $\mathrm{O} 3$ - use the refresh button

- O4 - upload files to another computer

- O5 - use back and forward buttons

- O6 - download programs from the Internet

- $\mathrm{O} 7$ - watch video files

- O8 - download music files

Formal Internet skills:

- F1 - use multiple browser windows

- $\quad$ F2 - find Web sites to be confusing

- F3 - get lost

- F4 - feel disoriented

- F5 - experience difficulties with a Web site's layout

- F6 - not know where you are

Information Internet skills:

- I1 - check information retrieved on another Web site

- $\quad$ I2 - examine only the top results

- $\mathrm{I} 3$ - search for information

- I4 - find the information you were looking for

- I5 - use advanced search options (e.g., Boolean operators)

- I6 - examine the results on subsequent result pages

- $\quad$ I7 - use more than one search keyword

- $\quad$ I8 - evaluate the source of the information found

Strategic Internet skills:

- $\quad \mathrm{S} 1$ - make a decision based on retrieved information

- $\quad$ S2 - use information about a specific subject from multiple sites

- $\quad \mathrm{S} 3$ - buy a product based on retrieved information 
- $\mathrm{S} 4$ - benefit from using the Internet

- $\quad$ S5 - reach your intended goal

- S6 - use reference Web sites

- $\quad$ S7 - gain financial benefits

Table 6.2 Pearson's Correlations between Items and the Number of Operational (O), Formal (F), Information (I), and Strategic (S) Internet Skills Assignments Completed Successfully and the Time Spent

\begin{tabular}{|c|c|c|}
\hline Agreement measures & $\begin{array}{c}\text { Successful Task } \\
\text { completion }\end{array}$ & Time spen \\
\hline I sometimes save files from the Internet & $.54^{* \star}$ & $-.54^{\star \star}$ \\
\hline Using the Internet browser is self-evident & $.53^{\star *}$ & $-.56^{\star \star}$ \\
\hline I often download music files & $.49^{* *}$ & $-.60^{\star *}$ \\
\hline I often use the back and forward buttons & $.45^{\star \star}$ & $-.51^{\star \star}$ \\
\hline I often do not now the purpose of the Internet browser buttons & $.44^{\star *}$ & $-.47^{\star \star}$ \\
\hline $\begin{array}{l}\text { I sometimes adjust the Internet browser settings to my personal } \\
\text { preferences }\end{array}$ & $.38^{\star *}$ & $-.48^{\star \star}$ \\
\hline I sometimes add a Web site to the "Favorites" & $.26^{\star}$ & $-.40^{\star *}$ \\
\hline I often use multiple browser windows at the same time & $.55^{\star \star}$ & $-.58^{\star \star}$ \\
\hline I sometimes find the Web site's design to be complex & $-.34^{\star *}$ & $.36^{\star \star}$ \\
\hline On the Internet, I often feel disoriented & $-.33^{\star *}$ & $.23^{\star}$ \\
\hline To me, the design of a Web site is often incomprehensible & $-.32^{\star *}$ & $.27^{\star}$ \\
\hline Web sites are sometimes confusing & $-.23^{*}$ & $.24^{*}$ \\
\hline On the Internet, I often get lost & $-.23^{\star}$ & $.22^{*}$ \\
\hline I sometimes do not know where I am on the Internet & -.15 & .19 \\
\hline All those different layouts make working with the Internet difficult & -.11 & .06 \\
\hline It is often difficult to retrieve a Web site on the Internet & -.10 & .18 \\
\hline I should follow a course for searching the Internet & $-.29^{\star \star}$ & $.35^{\star \star}$ \\
\hline I normally use more than one keyword when searching & $.26^{*}$ & $-.28^{*}$ \\
\hline On the Internet, I often do not find what I am looking for & $-.24^{*}$ & $.25^{\star}$ \\
\hline I normally examine more than just the top results & .17 & $-.34^{* *}$ \\
\hline $\begin{array}{l}\text { I sometimes do not know what search terms to use when } \\
\text { searching the Internet }\end{array}$ & -.17 & $.34^{\star *}$ \\
\hline I sometimes check information on another Web site & .03 & $-.26^{\star}$ \\
\hline I normally can easily choose from search results & .07 & $-.24^{*}$ \\
\hline I do not really care where information on the Internet comes from & -.10 & .10 \\
\hline I normally check the source of information on the Internet & .08 & -.12 \\
\hline I often gain benefits from using the Internet & $.31^{\star *}$ & -.10 \\
\hline Using the Internet brings me little benefit & $-.31^{\star *}$ & .09 \\
\hline The Internet sometimes saves me money & $.29^{\star \star}$ & -.12 \\
\hline Except pleasure, using the Internet brings me little benefit & $-.29^{\star \star}$ & .09 \\
\hline On the Internet, I often achieve my goals & $.24^{*}$ & -.05 \\
\hline When I have to make a choice, I sometimes consult the Internet & .20 & -.02 \\
\hline I sometimes make important decisions with the help of the Internet & .09 & -.12 \\
\hline
\end{tabular}




\subsection{Step 2: Discriminant validity of the Internet skills items}

Discriminant validity is the degree to which items differentiate between constructs or measure distinct concepts. The Fornell and Larcker criterion is satisfied when a construct is more closely related to its own indicators than to other constructs. Table 6.3 shows the correlation matrix of the items that resulted from step one. A careful examination of the correlations reveals that most of the four Internet skills items exhibit convergence. However, the rules for discrimination do not hold to indicate the existence of the operational, information, and strategic Internet skills constructs. A construct can be extracted only for formal Internet skills when applying the Fornell and Larcker discriminant validity criterion. For instance, the first eight items (O1 to O8), which represent the dimension of operational Internet skills, converge by exhibiting uniformly high correlations among themselves (with the exception of O5); however, they do not discriminate, since they also reveal high correlations with items in other dimensions, especially information Internet skills. In addition, several of the information Internet skills items have strong correlations with strategic Internet skills items (for example, items I2, I3, and I4 with items S1 and S3).

In general, no strict structure has appeared, and the rules of convergence and discrimination do not hold very strong over the four Internet skills dimensions. However, it is possible to extract items that have the highest potential for further analyses. The convergence is highest between seven of eight operational Internet skills items. These items are:

- O1 - save files

- $\mathrm{O} 2$ - complete forms

- O3 - use the refresh button

- O4 - upload files to another computer

- O6 - download programs

- $\quad$ O 7 - watch video files

- O8 - download music files

The internal consistency of these items, measured with Cronbach's $\alpha$ coefficients, is .76. This creates a new variable that yields correlation coefficients of $.55(\mathrm{p}<.01)$ and $-.59(\mathrm{p}<.01)$ for successful completion of the operational Internet skills tasks and for the time spent on these tasks, respectively. The mean of this new variable is $3.27(\mathrm{SD}=1.19)$. 


\section{Table 6.3}

Correlation Matrix of the Operational (O), Formal (F), Information (I), and Strategic (S) Internet Skills Items

\begin{tabular}{|c|c|c|c|c|c|c|c|c|c|c|c|c|c|c|c|}
\hline & 1 & 2 & 3 & 4 & 5 & 6 & 7 & 8 & 9 & 10 & 11 & 12 & 13 & 14 & 15 \\
\hline 1. 01 & - & .49 & .47 & .42 & .45 & .49 & .61 & .46 & .08 & 25 & .04 & .05 & .07 & .07 & .31 \\
\hline 2. $\mathrm{O} 2$ & & - & .42 & .40 & .25 & .31 & .37 & .34 & .02 & .04 & .12 & .00 & .00 & .11 & .24 \\
\hline 3. 03 & & & - & .48 & .35 & .36 & .37 & .39 & .21 & .19 & .09 & .08 & .03 & .07 & .30 \\
\hline 4. 04 & & & & - & .17 & .50 & .46 & .40 & .02 & .19 & .08 & .06 & .07 & .05 & .17 \\
\hline 5.05 & & & & & 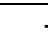 & .19 & .32 & .29 & .23 & .28 & .08 & .06 & .01 & .28 & .18 \\
\hline 6.06 & & & & & & . & .44 & .48 & .08 & .20 & .24 & .08 & .07 & .15 & .17 \\
\hline 7. 07 & & & & & & & - & .66 & .11 & .20 & .04 & .04 & .03 & .14 & .15 \\
\hline 8. 08 & & & & & & & & - & .04 & .08 & .14 & .06 & .05 & .12 & .03 \\
\hline 9. F1 & & & & & & & & & - & .36 & .32 & .32 & .30 & .28 & .07 \\
\hline 10. F2 & & & & & & & & & & - & .33 & .38 & .42 & .45 & .06 \\
\hline 11. F3 & & & & & & & & & & & - & .44 & .44 & .56 & .17 \\
\hline 12. F4 & & & & & & & & & & & & - & .39 & .42 & .06 \\
\hline 13. F5 & & & & & & & & & & & & & - & .60 & .02 \\
\hline 14. F6 & & & & & & & & & & & & & & - & .03 \\
\hline 15. $I 1$ & & & & & & & & & & & & & & & - \\
\hline 16. 12 & & & & & & & & & & & & & & & \\
\hline 17. 13 & & & & & & & & & & & & & & & \\
\hline 18. 14 & & & & & & & & & & & & & & & \\
\hline 19. 15 & & & & & & & & & & & & & & & \\
\hline 20. 16 & & & & & & & & & & & & & & & \\
\hline 21. 17 & & & & & & & & & & & & & & & \\
\hline 22. 18 & & & & & & & & & & & & & & & \\
\hline 23. S1 & & & & & & & & & & & & & & & \\
\hline 24. S2 & & & & & & & & & & & & & & & \\
\hline 25. S3 & & & & & & & & & & & & & & & \\
\hline 26. $\$ 4$ & & & & & & & & & & & & & & & \\
\hline 27. S5 & & & & & & & & & & & & & & & \\
\hline 28. S6 & & & & & & & & & & & & & & & \\
\hline 29. S7 & & & & & & & & & & & & & & & \\
\hline
\end{tabular}

Note: Significant at $p<.05$; non-significant correlations are in italic. 


\begin{tabular}{|c|c|c|c|c|c|c|c|c|c|c|c|c|c|c|}
\hline 16 & 17 & 18 & 19 & 20 & 21 & 22 & 23 & 24 & 25 & 26 & 27 & 28 & 29 & \\
\hline .43 & .40 & .41 & .38 & .37 & .46 & .20 & .18 & .11 & .48 & .22 & .10 & .18 & .33 & 1.01 \\
\hline .49 & .43 & .38 & .32 & .30 & .34 & .32 & .39 & .17 & .48 & .28 & .21 & .23 & .39 & 2. 02 \\
\hline .51 & .39 & .43 & .38 & .37 & .40 & .22 & .22 & .06 & .46 & .15 & .06 & .17 & .08 & 3. 03 \\
\hline .38 & .27 & .17 & .44 & .19 & .24 & .12 & .19 & .01 & .42 & .10 & .09 & .07 & .07 & 4. 04 \\
\hline .49 & .50 & .44 & .20 & .48 & .46 & .35 & .28 & .19 & .36 & .31 & .03 & .19 & .25 & 5. 05 \\
\hline .36 & .29 & .31 & .45 & .21 & .21 & .16 & .21 & .07 & .47 & .14 & .12 & .11 & .17 & 6.06 \\
\hline .35 & .46 & .29 & .37 & .24 & .45 & .13 & .13 & .04 & .36 & .25 & .10 & .17 & .21 & 7. 07 \\
\hline .34 & .29 & .20 & .32 & .17 & .43 & .23 & .20 & .03 & .37 & .06 & .14 & .12 & .17 & 8. 08 \\
\hline .16 & .01 & .25 & .06 & .18 & .11 & .03 & .16 & .10 & .17 & .11 & .03 & .05 & .04 & 9. F1 \\
\hline .22 & .17 & .29 & .03 & .16 & .11 & .16 & .01 & .06 & .15 & .01 & .15 & .06 & .12 & 10. F2 \\
\hline .18 & .17 & .30 & .03 & .08 & .03 & .22 & .06 & .04 & .15 & .15 & .07 & .10 & .07 & 11. F3 \\
\hline .02 & .07 & .11 & .13 & .04 & .14 & .04 & .05 & .05 & .00 & .08 & .01 & .01 & .06 & 12. F4 \\
\hline .10 & .01 & .12 & .21 & .05 & .14 & .03 & .09 & .07 & .10 & .09 & .08 & .01 & .03 & 13. F5 \\
\hline .05 & .25 & .28 & .10 & .14 & .13 & .13 & .06 & .00 & .08 & .03 & .04 & .03 & .07 & 14. F6 \\
\hline .35 & .26 & .40 & .39 & .37 & .24 & .04 & .23 & .19 & .14 & .32 & .05 & .26 & .28 & 15. I1 \\
\hline \multirow[t]{14}{*}{ - } & .57 & .49 & .31 & .65 & .52 & .35 & .54 & .27 & .62 & .31 & .07 & .28 & .36 & 16. 12 \\
\hline & - & .62 & .24 & .45 & .53 & .40 & .39 & .14 & .49 & .42 & .00 & .33 & .30 & 17. 13 \\
\hline & & - & .18 & .41 & .44 & .34 & .37 & .27 & .49 & .38 & .01 & .29 & .26 & 18. 14 \\
\hline & & & - & .31 & .25 & .22 & .20 & .05 & .36 & .23 & .13 & .22 & .08 & 19. 15 \\
\hline & & & & - & .50 & .38 & .36 & .17 & .33 & .24 & .11 & .26 & .20 & 20. 16 \\
\hline & & & & & - & .40 & .25 & .16 & .43 & .29 & .03 & .17 & .18 & 21.17 \\
\hline & & & & & & & .22 & .21 & .40 & .27 & .14 & .17 & .18 & 22. 18 \\
\hline & & & & & & & - & .32 & .51 & .50 & .15 & .55 & .38 & 23. S1 \\
\hline & & & & & & & & & .29 & .21 & .32 & .24 & .33 & 24. S2 \\
\hline & & & & & & & & & - & .35 & .06 & .25 & .32 & 25. S3 \\
\hline & & & & & & & & & & - & .12 & .36 & .29 & 26. $\$ 4$ \\
\hline & & & & & & & & & & & - & .23 & .12 & 27. S5 \\
\hline & & & & & & & & & & & & - & .55 & 28. S6 \\
\hline & & & & & & & & & & & & & - & 29. S7 \\
\hline
\end{tabular}


All six items regarding formal Internet skills show both convergent and discriminant validity. Item F1 (use multiple browser windows) was deleted to increase internal consistency. The remaining items are:

- F2 - find Web sites to be confusing

- F3 - get lost

- F4 - feel disoriented

- F5 - experience difficulties with a Web site's layout

- F6 - not know where you are

These items together have a Cronbach's $\alpha$ coefficient of .77. This creates a new variable that yields correlation coefficients of $.31(\mathrm{p}<.01)$ and $-.25(\mathrm{p}<.01)$ for successful completion of the formal Internet skills tasks and for the time spent on these tasks, respectively. The mean of this new variable is $2.53(\mathrm{SD}=0.87)$.

Six of the eight items regarding information Internet skills show moderate convergence. These include the following items:

- I1 - check information retrieved on another Web site

- I2 - examine more than one search result

- I3 - search for information

- I4 - find the information I was looking for

- I6 - examine more than one page of search results

- $\quad$ I7 - use more than one search keyword.

The internal consistency of these items, measured with Cronbach's $\alpha$ coefficients, is .82. This creates a new variable that yields correlation coefficients of .39 $(\mathrm{p}<.01)$ and $-.27(\mathrm{p}<.01)$ for successful completion of the information Internet skills tasks and for the time spent on these tasks, respectively. The mean of this new variable is $3.27(\mathrm{SD}=1.19)$.

Regarding strategic Internet skills, five of the seven items show moderate convergence. These include the following items:

- $\mathrm{S} 1$ - make a decision based on retrieved information

- S2 - use information about a specific subject from multiple sites

- S4 - benefit from using the Internet

- S6 - use reference Web sites

- S7 - gain financial benefits

The internal consistency of these items, measured with Cronbach's $\alpha$ coefficients, was .76. This creates a new variable that yields correlation coefficients of .46 $(\mathrm{p}<.01)$ and $.14(\mathrm{~ns})$ for successful completion of the strategic Internet skills tasks 
and for the time spent on these tasks, respectively. The mean of this new variable is $3.32(\mathrm{SD}=1.16)$.

\subsection{Step 3: Internet skills items in a first-order confirmatory factor analysis}

In the third step of the analyses, the 23 remaining items from step 2 are further validated in a first-order confirmatory factor analysis. In order to perform this analysis, a study was conducted in which the 23 items were included in the pilot test of a large scale survey concerning Internet access and use. Subscribers of a national online panel (administrated by a profit research and consultancy company) were invited via email to voluntary participate in this online survey in August 2010. In total, 300 subscribers were invited and a total of 238 completed surveys were returned. A brief overview of the respondents is provided in Table 6.4 .

Table 6.4 Subjects over Gender, Age, and Education

\begin{tabular}{llrr}
\hline & & $n$ & $\%$ \\
\hline Gender & & & \\
& Male & 157 & 66 \\
& Female & 81 & 34 \\
\hline Age & & & \\
& $18-29$ & 5 & 2 \\
& $30-39$ & 19 & 8 \\
& $40-54$ & 75 & 32 \\
& $55-80$ & 138 & 58 \\
\hline Education & Low (e.g., Primary school) & & \\
& Middle (e.g., High school) & 42 & 18 \\
& High (e.g., College and University) & 77 & 32 \\
&
\end{tabular}

Prior to the analyses, data were checked for normality. Because of skewness to the lower end of the distribution of the formal Internet skills measures, an inverse (reciprocal) transformation was performed to correct skew (Garson, 2006). Using a first-order confirmatory factor analysis, the measurement model estimated the extent to which the 23 observed items loaded onto their respective latent variables. All latent constructs but no observed error variances were allowed to covary with one another. The initial measurement model generated poor fit (for required fit measures, see Section 4.6), $\chi^{2}(224)=557.81, \chi 2 / \mathrm{df}=2.49, \mathrm{SRMR}=$ 
.087, TLI $=.831$, RMSEA $=.079(90 \%$ confidence interval $[\mathrm{CI}]: .071, .088)$. Subsequently, items with highly correlated error variances identified by post hoc modification indices and items that loaded poorly onto its unique factor were removed. This procedure resulted in the reduction of the number of observed indicators of the latent constructs to better fit the measurement model. Two operational Internet skills items (O2 - complete forms, and O8 - download music files) and one information Internet skills item (I3 - search for information) were removed. The internal consistency of the measures were above aspiration level $(\alpha$ $>$.70). The modified measurement model generated a good fit, $\chi^{2}(164)=284.01$, $\chi^{2} / \mathrm{df}=1.73, \mathrm{SRMR}=.061, \mathrm{TLI}=.923, \mathrm{RMSEA}=.056(90 \%$ confidence interval [C] : .045, .066).

Table 6.5 Descriptive Statistics, Factor Loadings, Squared Multiple Correlations, and Cronbach's a's for the Observed Internet Skills Indicators

\begin{tabular}{lllll}
\hline & $M$ & $S D$ & $\beta$ & $R^{2}$ \\
\hline Operational Internet Skills ( $\mathrm{a}=.73)$ & & & & \\
O1 - save files & 2.53 & 1.30 & .60 & .36 \\
O3 - use the refresh button & 3.38 & 1.57 & .57 & .33 \\
O4 - upload files to another computer & 3.96 & 1.22 & .66 & .43 \\
O5 - download programs & 3.99 & 1.10 & .61 & .38 \\
O7 - watch video files & 3.00 & 1.19 & .53 & .29 \\
Formal Internet Skills ( $\mathrm{a}=.79$ ) & & & & \\
F2 - find Web sites to be confusing & 1.74 & 0.91 & .71 & .57 \\
F3 - get lost & 1.34 & 0.68 & .80 & .64 \\
F4 - feel disoriented & 1.49 & 0.76 & .69 & .48 \\
F5 - experience difficulties with a Web site's layout & 1.86 & 0.88 & .51 & .26 \\
F6 - not know where you are & 1.47 & 0.88 & .62 & .38 \\
Information Internet Skills ( $\mathrm{a}=.87$ ) & & & & \\
I1 - check information retrieved on another Web site & 2.86 & 1.01 & .84 & .70 \\
I2 - examine only the top results & 2.05 & 1.01 & .74 & .55 \\
I4 - find the information you were looking for & 1.97 & 1.06 & .75 & .56 \\
I6 - examine the results on subsequent result pages & 1.96 & 0.95 & .86 & .74 \\
I7 - use more than one search keyword & 1.97 & 1.06 & .67 & .45 \\
Strategic Internet Skills ( $a$ = .81) & & & & \\
S1 - make a decision based on retrieved information & 3.49 & 1.14 & .65 & .43 \\
S2 - use information about a specific subject from multiple sites & 3.17 & 1.08 & .60 & .36 \\
S4 - Benefit from using the Internet & 2.97 & 1.14 & .58 & .33 \\
S6 - use reference Web sites & 3.42 & 0.99 & .83 & .54 \\
S7 - gain financial benefits & 2.51 & 1.23 & .74 & .69 \\
\hline
\end{tabular}

Table 6.5 summarizes the original (uncorrected) means and standard deviations, Cronbach's alphas, the factor loadings $(\beta)$, and the squared multiple correlations $\left(\mathrm{R}^{2}\right)$ of the observed indicators. The correlation matrix of the observed variables is shown in Table 6.6. 


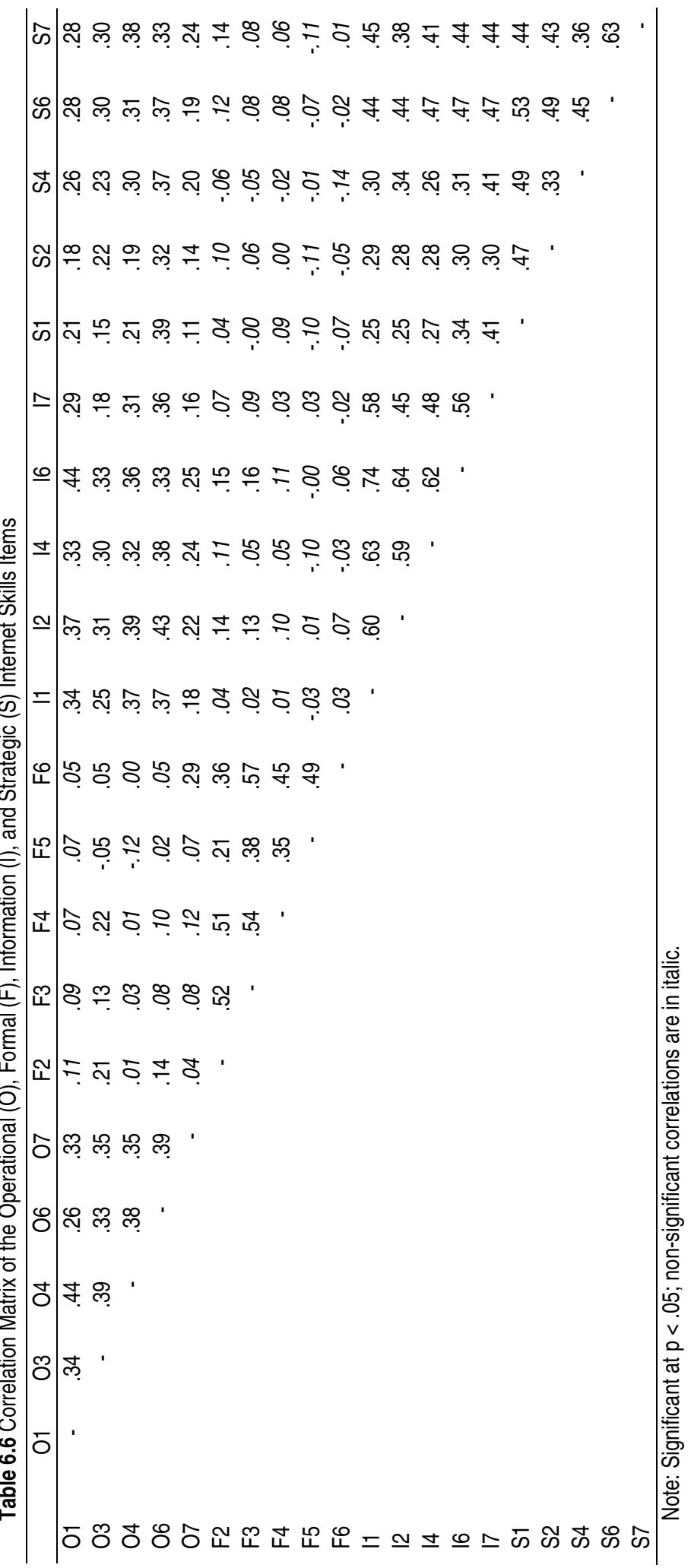




\subsection{Conclusions}

This chapter analyzed potential survey measures for the four Internet skills. Three steps were followed to obtain valid items. In the first step, correlations between frequency scales and the actual performances are measured. This step revealed that some of the items proposed might serve as proxies for Internet skills. In the second step, the Fornell and Larcker (1981) discriminant validity criterion was used to test discriminant validity of the remaining items. In the third step the items were further analyzed using a first-order confirmatory factor analysis based on a new data sample. The items that resulted from the three steps might be used in future survey measures and are shown in Table 6.5. Table 6.6 reveals that it is possible to observe the four Internet skills separately, hereby confirming on the theory discussed in Chapter 3. Three problems should be addressed in further analyses:

1. No clear pattern emerges when the items are analyzed. The desired outcome from discriminant validity criteria is that four different Internet skills constructs would have appeared, as proposed in the Internet skills definition. The analyses revealed that although several specific Internet skills items reveal strong convergence, they also show significant high correlations with items from other Internet skills. This can partly be explained by the nature of the four Internet skills, which is sequential and conditional. Although this specific nature suggests significant correlation between the four Internet skills items, still stronger correlations between the items of one specific skill rather than between different Internet skills items would have been the ideal result.

2. Table 6.5 reveals that most of the items reveal only moderate squared multiple correlations (aspiration level is $>.05$ ) which indicates that they are especially explaining themselves, instead of the latent construct they belong to. A stronger operationalization of the skill items is needed. Therefore extended item batteries should be developed to obtain more discriminant validity.

3. Despite the significant correlations, the mean scores of the four Internet skills constructs do not reflect the scores of the actual performance tests. In fact, the mean score of the strategic Internet skills items is higher than the mean scores of the information Internet skills. Since the observations in the performance tests are more accurate, this result indicates that people have serious trouble indicating their own frequency of specific 
skills-related actions. Further research should carefully test, if at all possible, to obtain valid data by using surveys concerning information and strategic Internet skills. 


\subsection{Introduction}

The main goal of this dissertation was fivefold: (1) to increase our knowledge of the role Internet skills play in contemporary society, (2) to develop an Internet skills definition that can be used in measurements, (3), to indicate the level of Internet skills among the Dutch population, (4) to propose a survey instrument for measuring Internet skills, and (5) to provide recommendations for improving Internet skills levels and Web site development. Several research questions were framed to achieve these goals. Research Questions 1 to 9 are separately addressed in the prior chapters. Before addressing Research Question 10 in the next chapter, first general conclusions and discussions are put in a larger perspective.

The dissertation started out with a brief historic overview. In this overview, it was substantiated that besides increasing demands put on people due to advancements in communication technology, at the same time also the stakes for not being able to keep up have risen. Failing to sustain the changes in communication technology is more and more likely to result in serious disadvantages, eventually resulting in a reinforcement of existing social inequalities. This is most apparent in the contemporary information society, in which it is increasingly necessary to have the skills to make the most out of Internet usage. Since the Internet and the information it represents now play an important role in political, economic, social, and cultural domains, Internet skills should be considered vital assets.

Internet skills were measured by applying an elaborate definition that goes beyond button knowledge or technical skills. The proposed definition distinguished between operational and formal (or medium-related) Internet skills and information and strategic (or content-related) Internet skills. To date, surveys have mainly addressed operational skills and, to some extent, formal skills, thereby providing an overly positive overall picture. The main benefits of the proposed definition are the four types of Internet skills separately distinguished by others in the literature and the sequential and conditional nature of these four types of skills. The definition considers functional skills that are a requirement for typical Internet use, common to the majority of Internet users. Three observational studies were conducted in which subjects were given assignments on the Internet. The results provided greater insight into the actual level of Internet skills of the Dutch population and showed that the proposed Internet skills definition is a 
powerful means for understanding the complex sequential and conditional nature of Internet skills.

In this chapter, first conclusions about the actual level of Internet skills are drawn. Concluding remarks about the differences in Internet skills among several segments of the Dutch population are then provided. From these differences, the consequences of not having sufficient levels of Internet skills are further discussed in the realm of social inequality as introduced in Chapter 2. The chapter ends with some final recommendations for future research.

\subsection{Level of Internet skills}

The three performance tests were conducted between 2007 and 2010. Their primary goal was to indicate the level of operational, formal, information, and strategic Internet skills of the Dutch population. All three studies used a different context for the created assignments subjects had to complete on the Internet. The assignments to measure Internet skills were related to the government in the first study, to general leisure in the second study, and to health in the third study. In all three domains, a large amount of information and services are offered online. The results of the three studies were similar. A large number of assignments were not completed successfully.

When differentiating between the types of Internet skills, the conducted studies show that the level of operational and formal Internet skills in general appeared quite high. Although the number of people between 21 and 40 years old was relatively high in the performance tests when compared to the general Dutch population-which means that the results regarding the level of these skills should be moderated - it is safe to say that the level of these skills are the highest, especially when compared to the level of information and strategic Internet skills. The latter leave much room for improvement. It is important to understand that operational and formal Internet skills are not sufficient for effective use of the Internet and that information and strategic skills are more problematic. The assumption that assistance can always be provided to those that have insufficient skills might be partly true for relatively basic operations, but certainly not for more complicated ones that require information and strategic Internet skills. 
A qualitative analysis of the results of the first performance tests revealed some important findings. Worrisome is the fact that many subjects were unable to save or even open PDF documents. These are often offered online, sometimes as replacements of their printed counterparts. The basic operations to handle the Internet, such as typing a URL in the address bar or typing search queries in the search bar, are not self-evident for all Internet users either. Furthermore, several subjects experienced problems when new browser windows were opened, something not unusual on the Internet. The fact that every Web site employs its own layout and menu structures does not make things easier. Regarding information Internet skills, it became clear that users experience many problems in all steps of the information-seeking process. They have trouble defining proper search queries and selecting relevant search results. Most worrisome, however, is the fact that nobody seems to evaluate information found on the Internet. This became especially clear when some subjects gathered official government information from a Web site containing lectures of primary school students. Results regarding strategic Internet skills revealed that almost all subjects experienced trouble working in a goal-oriented way, using reference Web sites, and making decisions based on multiple information sources.

From a statistical point of view, the number of subjects that participated in the three performance tests is not sufficient to draw conclusions about the entire Dutch population. However, approximately 300 subjects participated in the performance tests, which provides a fairly good indication of the Internet skills levels of the Dutch population, as much trouble was taken to reach sample dispersion. Testing a random sample of approximately 1200 individuals from the entire Dutch population was unrealistic because of the laborious nature of performance tests. To rate the overall representativeness of the sampling approach, it should be compared to the standards of an experiment rather than a survey. For an experiment, the number of subjects is high. However, larger than average experimental groups were required because large social and cultural differences in computer use and experience had to be taken into account.

To our knowledge, there are no other performance tests of users' Internet skills that explicitly distinguish between different Internet skills in a conditional and sequential manner. Unfortunately, this means that there are no direct standards of comparison within the Netherlands or in any other country. When putting the results in a larger perspective, five general claims can be made: 
1. The Netherlands is a country with very high household Internet penetration (93\% in 2010) and a high level of educational attainment. It is to be expected that performance in many other countries in the world will be lower.

2. Evidently, the results depend on the difficulty of the assignments provided to the subjects. In the first study, relatively simple tasks of using online government information were selected. In the second study, an even more feasible context was used, as virtually everyone is familiar with the leisure activities used for the Internet assignments. Finally, in the third study, health-related assignments that are accessible to the general population were used. The results of all three studies appeared similar, suggesting that previously existing knowledge about government issues or health seems to have had a minor effect on the level of Internet skills.

3. The three studies were administered to Dutch-speaking subjects only. Since the amount of content available in other languages, especially English, is larger than available content in the Dutch language, employing information and strategic Internet skills in these languages might be even more difficult. However, this assumption should be tested further in future research.

4. In actual Internet use outside the artificial test situation, performances can expected to be lower. In a test situation, subjects are often more motivated to complete an assignment (though, in this case, they were not explicitly spurred). In their own environments, many of them would have grabbed the phone or run to a service desk or someone else in their social environment to get the answer. Other research among the Dutch population indicates that users of public Web sites often give up and turn to the telephone or a front desk (Pietersen \& Ebbers, 2008). However, one might also argue that specific technicalities used disadvantaged the subjects, especially concerning operational Internet skills, since these are most likely to be influenced by specific settings. Information and strategic Internet skills are less likely to be influenced, since the use of Google, for example, is not browser specific. Furthermore, the subjects could choose between the most common Internet browsers to replicate their normal Internet behavior. However, commanding the skills to use an Internet 
browser without specific personal settings might also be considered an aspect of operational Internet skills.

5. The assignments created to measure information Internet skills "helped" subjects by providing the first stage of the information search process. By defining the assignments, the problem definition to some extent is already accounted for.

By working outside the usual setting, the subjects were also prevented from asking help from peers, something they might have normally done. This became especially apparent when one of the subjects suddenly started to call his brother on his mobile phone (to ask how to go back to the homepage of the Dutch Tax and Customs administration). The results of all three performance tests, however, revealed minimal effects of social support when determining Internet skills levels. The level of Internet skills was not influenced by whether these subjects relied on peers when using the Internet. However, it might be a valuable addition in future research to include observational methods in real-life settings. From the results of the performance tests, for example, one wonders whether people that need help have a sufficient social network to look to for assistance, especially concerning information and strategic related questions.

The results of the performance tests force policy makers and new media developers to adjust their beliefs that, with the exception of some seniors, everybody has access to and can use the Internet. When considering physical access to the Internet, it seems that Dutch national figures are reaching the saturation phase; in 2010, 93\% of individuals 16 years and older had physical access to the Internet (CBS Statline). The results of the Internet skills performance tests now add that a large part of those that actually do use the Internet possess low levels of Internet skills, particularly the content-related information and strategic Internet skills. Based on these results, one might seriously question whether a population-even in a country with such high levels of Internet penetration and broadband connections in comparison with other countries-is ready for the migration of many informative pages and services to the Internet. There seems to be much room for improvement in terms of Internet skills.

The results of the conducted studies strengthen the findings that the original digital divide (defined as the gap between people who have and do not have 
physical access to a computers and the Internet) has developed a second divide that includes differences in the skills to use the Internet. The results indicate that a large part of the Dutch population is struggling to equip themselves with the skills they need to fully participate in contemporary society. Although the lack of operational and formal Internet skills can be considered a temporary problem (until a better accessible technology appears), the lack of information and strategic skills appears to be more structural in nature. For these skills, it will be much more difficult to reach full dispersion over the population. These skills strongly relate to education and intellectual capacities and should therefore gain a more central position in future research. While originally, the digital divide could be "easily" addressed by providing physical access, by focusing on the content-related skills, one might seriously argue that closing the divide here is like reaching for the moon. However, the following chapter will provide recommendations to improve all four Internet skills levels.

\subsection{Differences in Internet skills}

In the digital divide discourse, scholars often focus on the social demographic features along which differences in Internet use develop. The results of the conducted performance tests reveal that age and educational level of attainment are the most important contributing factors to level of Internet skills. Regarding age, the findings revealed some major implications. In contemporary society, the younger generations are associated with frequent and confident use of the Internet (Hargittai, 2010). They are considered unproblematic Internet users that can easily keep up with advancements in communication technologies. Conversely, the older generations are considered problematic and highly unconfident Internet users. The results of the actual performance tests, however, prove different, at least for those over 18 years of age. It appears that higher age only contributes negatively to the level of medium-related operational and formal Internet skills. Seniors, in particular, show low levels of these skills. However, although young people perform well on the medium-related Internet skills, they still show a strikingly low level of information and strategic Internet skills. In fact, it was shown that age has a direct positive effect on content-related skills, meaning that older people perform better on these skills than young people. Unfortunately, older people have considerable problems with the operational and formal Internet skills that strongly influence their performance on the information and strategic Internet 
skills. A popular and unpleasant assumption is that with the passing away of the oldest age groups the Internet skill problem will solve itself (Loos, 2010). This assumption should be countered and seems to be primarily based on the level of operational and formal Internet skills. Unfortunately, this assumption is further strengthened by the seniors themselves. When they are asked to assess their own level of Internet skills, older people score lower than younger people do. People who have little confidence in their ability to use the Internet, who are dissatisfied with their Internet skills, or who are uncomfortable using the Internet may be said to have weak self-efficacy beliefs (Eastin \& Larose, 2000). Unfortunately, those with low levels of self-efficacy are less likely to adopt and use the Internet in the future than those with high degrees of self-efficacy (Eastin \& Larose, 2000). Furthermore, society also contributes to the offensive view that older people are awkward users of technology. Take, for example, commercials in which seniors are presented as clumsy. It seems necessary that this stereotype be carefully reconsidered to encourage older people to take up new technologies. Additionally, in most cases, younger people seem unable to benefit from communication technologies such as the Internet. In fact, younger people should take valuable lessons from older people instead of vice versa. Fortunately, in educational settings, the results regarding age are slowly becoming realized. Conducted research within this field found that students use the Internet at a relatively young age but lack the skills of reflection regarding search results and of critical Web site reading (e.g., Kuiper, 2007; Lorenzen, 2001; Metzger et al., 2003; Pritchard \& Cartwright, 2004; Shenton \& Dixon, 2003).

Regarding the effects of age, it must be emphasized that the obtained results account for the current era and current generations. It is not known whether the same differences will persevere in the future. Although operational and formal skills are easiest to account for, they might persist, since technology changes, and with these changes, new specific operational and formal skill-related problems will come into existence. Information and strategic Internet skills are more likely to be determined by educational levels in the future. When younger people are trained in these skills at school, they might become better at these skills. In future measurements, subjects under the age of 18 should also be included. It can be expected that, to a certain extent, they possess sufficient levels of operational and formal Internet skills but insufficient information and strategic Internet skills, something that should be addressed at an early age (see Chapter 8). 
When searching for an explanation for the finding that age is a negative contributor to the medium-related skills and a positive contributor to the levels of content-related Internet skills, additional intellectual skills that are needed to master information and strategic Internet skills are likely to appear. The high levels of operational and formal Internet skills observed among young people do not guarantee the development of these additional skills. The most important factordetermining all types of Internet skills - is the level of educational attainment. The higher educated the subject, the better they perform on operational, formal, information, and strategic Internet skills. Research within the digital divide discourse is often marked by differences in educational attainment, especially concerning Internet access. Katz and Rice (2002) argue that lower educated groups are unable to apply the content provided by the Internet to their functional needs. Although low general literacy levels are often related to digital inequality (Groot, Maassen, \& Brink, 2006), regarding Internet skills, another assumption is very common. It is often argued that people learn Internet skills more in practice, by trial and error, than in formal educational settings (De Haan \& Huysmans, 2002c; Van Dijk, 2005). From the results of the three studies, it is possible to conclude that this mainly goes for operational and formal Internet skills, but not for information and strategic skills. This makes education in information and strategic Internet skills the sine quo non of social importance in contemporary society.

Another conclusion that can be drawn from the performance test results is that the level of Internet skills has a weak relation with years of Internet experience and amount of time spent online. Internet experience only contributes to the level of medium-related Internet skills. It appears that information and strategic Internet skills do not grow with years of Internet experience or amount of time spent online weekly. Related survey research that uses Internet self-efficacy as a dependent variable often finds prior Internet experience to be a strong predictor. The fact that this only counts for operational Internet skills furthermore enervates the assumption that with the passing away of seniors, the Internet skill problem will solve itself. Regarding information and strategic Internet skills in particular, it is the educational level of attainment that is most relevant. The finding that information and strategic Internet skills do not grow with years of Internet experience or amount of time spent online can be explained by the possession of general intellectual skills that strongly relate to information and strategic Internet skills. These skills do not improve from long or heavy Internet use. The relatively 
weak relation of Internet experience and time spent online with operational and formal skills can also be explained. People often keep repeating similar mistakes when using the Internet. Computer users tend to rely on acquired skills, even when they are aware that they could learn more efficient procedures for achieving the same results (Cahoon, 1998). This probably also accounts for Internet use. People learn by trial and error, but when they more or less achieve the goals they had in mind, people will persist in making the same mistakes online. This certainly is the case when there is no one around to point out mistakes.

All other factors accounted for in the performance test only slightly contributed to the level of Internet skills. For some of these factors, this can probably be explained by their relation with educational level of attainment (e.g., socioeconomic status). Regarding gender, some important findings should be highlighted. Although self-assessments prior to the performance tests revealed that men consider their own level of Internet skills higher than women do, gender did not reveal any differences in the three performance tests. Since education plays such a significant role when considering Internet skills, the lack of significant results for gender is likely to be explained by the fact that, in the Netherlands, gender differences within education have largely disappeared.

\subsection{Internet skills, vital assets in an information society}

Before linking the levels of Internet skills to social inequality, it is important to understand what is actually new about the finding that educational level of attainment is an important factor for explaining the level of Internet skills. After all, differences in educational level of attainment have always been one of the causes of differences in society and opportunities in life. From this point of view, one might argue that the Internet is just the next advancement in communication technology with its usage determined by education. The Internet's information features are used in ways similar to those in which print and audiovisual media are employed. The acts of searching, selecting, processing, and evaluating information from online video, images, sounds, texts, and figures largely correspond to the information skills used with traditional media. People who consider inequality as a necessity for economic development would not be shocked by the observed Internet skills differences that are largely determined by education. Yet, the 
situation regarding the Internet is different and does require considerable attention. First, the Internet differs from other media in terms of communication capacities. Second, society and the role of information have changed. Both arguments are discussed below.

The Internet differs in the communication capacities of accuracy, storage capacity, and selectivity, which are far greater than in any other medium. Since the information provided by the Internet is infinite, more pressure is put on contentrelated information and strategic skills than in the use of other media. These differences imply that Internet skills increase the inequalities observed in the possession of traditional media skills. Although the Internet makes things easier, as it enables systematic simultaneous information retrieval from innumerable sources, it also makes information-seeking and gaining benefits from Internet use more difficult, because it assumes a number of new operational and formal skills to begin with. These skills raise an extra barrier in addition to the skills of reading and writing. Additionally, the Internet requires particular information and strategic skills that are partly different from those required for the use of traditional media.

Although the possession of information was central in all societies, it now seems to dominate the economy as well as human activities. In contemporary society, information sharing has become the major method of earning a living for large segments of society. Information and network society theory both acknowledge the importance of information as a vital resource in society. Coping with the massive amounts of available information and the supporting technologies is now important for everyone, more than ever before. This makes information and strategic Internet skills very important in contemporary (and future) information society. They will largely determine people's positions in the labor market and in social life.

If people with low levels of Internet skills fail to find information online while an increasing number of information and services relevant to daily life become easiest to access on the Internet, they become increasingly disadvantaged. What the exact contribution of Internet skills is here can only be determined when Internet skills are added as independent instead of dependent variables in future research. Then the consequences of Internet skills in terms of inequality can really be assessed among populations at large. Within this dissertation, survey measures for the four types of Internet skills are provided. When these measures are further validated, a 
clear picture of the exact influence of Internet skills on inequality might appear. However, from the results of the performance tests, some important conclusions concerning social inequality can be made.

In political, social, cultural, health, and economic domains, a wide and persistent migration towards the Internet can be observed. More and more information pages and services are provided online, and often, it is expected that they are accessible for all. In some cases, these services and information pages are offered with the intention of replacing traditional offline services, infringing basic human rights when people have no access to the Internet. The results of the studies reveal that within several domains, current policy directions should be evaluated, since there are strong indications that large parts of the population will be excluded from actual and effective Internet use. This especially goes for less educated populations. While these groups have always been socially disadvantaged, their life chances are now further in danger. They are shut out from economic opportunities such as wage premiums, privileged access to jobs, and cheaper products. They are excluded from social opportunities such as intensified social interactions that result in higher life satisfaction. They are shut out from health opportunities such as better diets, improved exercise habits, better care, anonymous help, detection of potential medical problems, and collaborative treatment of illnesses. They are excluded from political opportunities such as online services and participation. Moreover, they are denied cultural opportunities such as online learning and online entertainment. This exclusion appears over all age groups within society. In the beginning, groups with fewer Internet skills will be persuaded in negative ways; flights will be booked, concerts will be sold out, jobs will be given away, and dates will primarily be granted to those having access. It is probably the people on the right side of the debate, mainly the higher educated, that directly notice the benefits and the gains they get from sufficient Internet skills. However, continuing the transformations towards the Internet in the most important domains of life will eventually lead to serious problems if the lack of Internet skills among large parts of the population is not accounted for. A further strengthening of the higher educated and weakening of the lower educated might ultimately even result in the existence of first-, second-, and third-class citizens. Inequalities in terms of educational differences have always existed. Increasingly, however, these inequalities should be perceived as injustice. This calls for immediate attention. A wise point of departure here might be the capability approach of Sen (1985). Since Internet skills are considered as vital 
assets in the contemporary information society-they affect economic, political, social, health, and cultural domains - and can be considered a booster for social inequality, policies should at least attempt to compensate for low levels of Internet skills, especially for those most in need. Unfortunately, the fact that educational differences will always exist makes an equal diffusion of sufficient levels of Internet skills among the population almost sound like utopia. This is especially true if one fails to acknowledge that the skills needed for the Internet add to the differences observed in traditional media. Then, it is unlikely new educational policies are suggested.

\subsection{Limitations and future research}

While other definitions of Internet skills often focus on specific aspects of Internet use, here, a definition of Internet skills is used that is derived from multiple research directions. The different skills are subsequently arranged in a particular order. The definition has three important characteristics:

1. The definition of Internet skills has a sequential and conditional nature. The results of the study emphasize the value of this peculiar distinction by revealing that information and strategic Internet skills, in particular, appear to be the most difficult in performance tests and leave much room for improvement.

2. The definition concentrates on task- and goal-oriented Internet use. This goes beyond the more traditional definitions of media literacy by suggesting a more (inter)active use (consisting of interaction with programs or people, transactions in goods and services, and making decisions).

3. The proposed Internet skills should be commanded by every Internet user if they want to effectively use this medium at all. The assignments administered to the subjects were assignments that all parts of the population are practicing on the Internet. Certain advanced skills (that might be desirable but are only possessed by a relatively small minority of the population) are neglected. 
The applied Internet skills definition, however, is not complete. The Internet skills definition used throughout this dissertation and in the actual measurements limits the content-related part of Internet skills to information. Information-related activities remain the most widely used (Witte \& Mannon, 2009). However, the communication capacities of the Internet have gained considerable attention over the last few years. E-mail, chatting, social networking, and online discussions are all important activities on the Internet. Performing these activities requires additional Internet skills. These could be called communication Internet skills, required for computer-mediated communication. These skills would then, for example, be the skills to communicate in online or virtual environments that are accompanied by a reduction of non-verbal behavior cues, the skills to handle patterns of asynchronous communication, or the skills to handle a flood of messages that are unknown in many traditional media. This all suggests that adding communication skills to the conceptual definition of Internet skills requires the adoption of particular theories of communication.

Besides communication Internet skills, other additions in future research are content creation and content sharing. These activities refer to so-called Web 2.0 applications. Although these skills have been ignored, it can be expected that both information and strategic Internet skills are crucial for these activities, even more than for information retrieval. Active participation and user-generated content require a high level of Internet skills, particularly for "serious" applications (as opposed to entertainment applications). In the studies reported in this dissertation, communication skills and skills for content creation were not measured, because this would have made the performance tests, which already required 1.5 hours from the subjects, an unrealistic effort.

Although we have compared traditional media skills with digital media skills, whether the observed lack of information and strategic skills appears to the same extent in the information retrieval of more traditional media and channels is yet to be empirically investigated. Further research should address this question by including comparable information and strategic skills performance tests in the use of media other than the Internet. A comparison of the results of all these tests could show whether the use of the Internet channel makes a difference and whether Internet use produces better information and better test results or introduces another barrier because many people do not master the special skills required for appropriate use of the Internet. Since computers and the Internet 
make things easier, as they enable systematic information retrieval from innumerable sources simultaneously, finding information in a traditional library might be more difficult for inexperienced information seekers than finding the same information on the Internet using a "simple" search engine. However, as discussed in Section 3.5, computers and the Internet make information seeking and improving literacy more difficult, as they assume a number of new operational and formal skills. This raises yet another barrier in addition to the skills of reading and writing. Furthermore, they require particular information-seeking and strategic skills that are somewhat different from those required for the use of traditional media. Confirmative research is necessary to reveal whether operational, formal, information, and strategic skills together increase the gap between people of differing ages and educational and occupational backgrounds in terms of the new (as opposed to traditional) media.

As already suggested in the prior sections, it is important to expand Internet skills measurements internationally. This will provide more detail on the absolute levels of Internet skills of specific populations and will also reveal whether there are differences in Internet skills and Internet skills determinants between societies.

Of the independent variables tested for when measuring Internet skills, two variables that are usually included in research concerning the digital divide are missing: income and race. Income seems to be more relevant concerning physical access to the Internet. The subjects included in the performance tests all had physical Internet access at home (and used broadband connections). Even if income restricted the subjects' amount of Internet use or Internet experience, the results of the performance tests revealed that these two variables only had a minor influence on the level of Internet skills. Furthermore, if income were to affect Internet skills, then this would probably mainly be due to differences in education and socio-economic position. Race was excluded, since the recruited sample did not contain enough immigrants to include them as a separate group in the analyses. It was impossible to include more subjects in the sample so that racial differences could also be accounted for, as the performance tests (two hours of data collection and at least two hours of intensive analysis) were very labor intensive. However, future studies should also include enough racial and ethnic minorities so that inferences can be drawn about their levels of Internet skills. 
In this dissertation, a new definition for measuring Internet skills is proposed. This definition proved suitable for making in-depth measurements of Internet skills. However, the next step is to move the proposed skills from dependent to independent variables in digital divide research. Although, in this dissertation, the influence of these skills on social inequality is theoretically discussed, empirical evidence should support these suggestions by assessing the input of Internet skills on Internet usage, social inequality, and different aspects of participation in society. Then, the exact impact of these skills will become apparent. Evidently, this requires further validation of the proposed Internet skills items discussed in Chapter 6. Furthermore, the use of longitudinal designs would improve the strength of the findings concerning effects of Internet skills. 

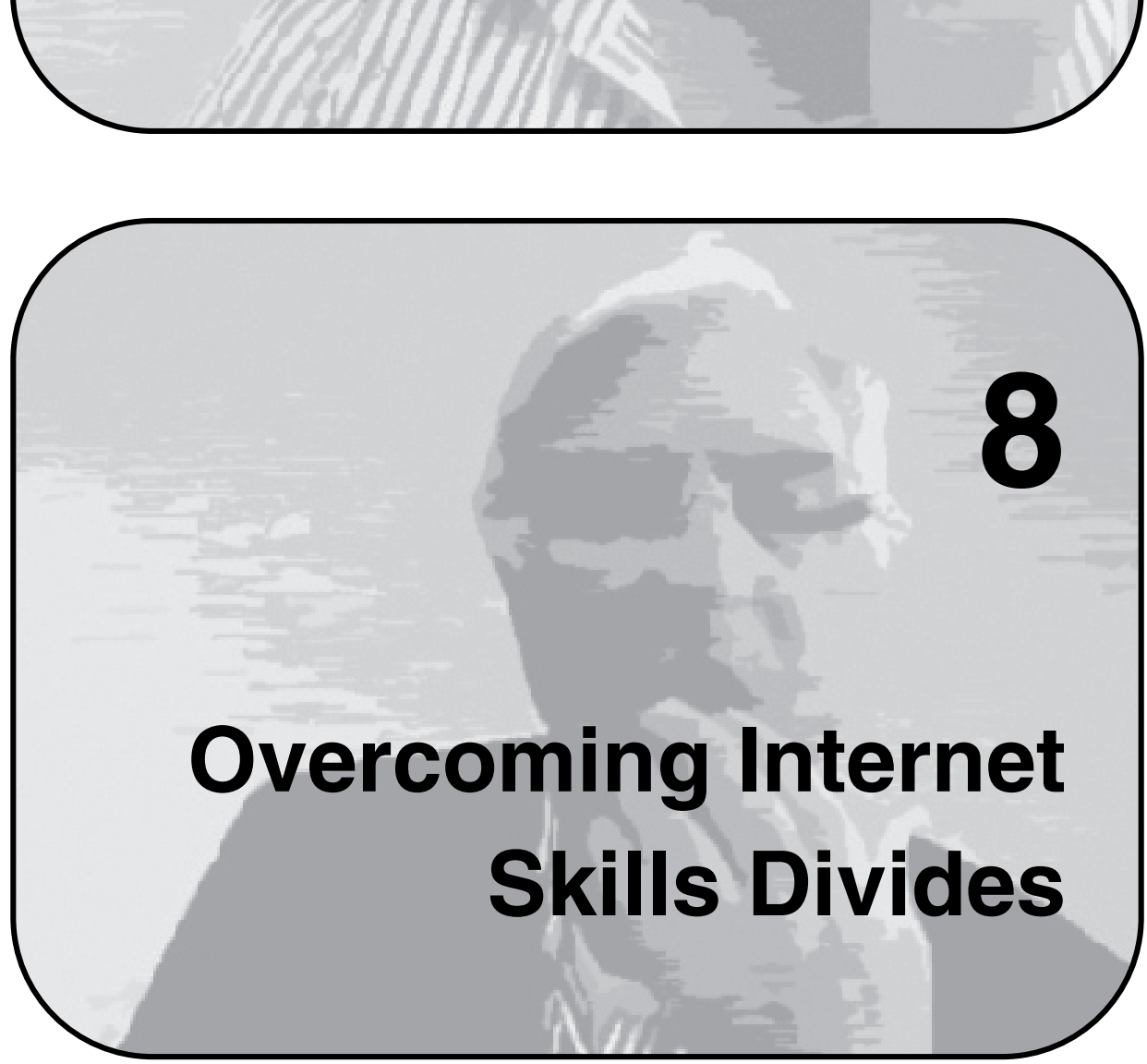

In this final chapter, two strategies for improving Internet skills levels are proposed. First, a supply-side oriented strategy is discussed. Here, it is important to improve the accessibility and usability of online information and service provision, improving Web sites in such a way that they better cater to users. In the second part of this chapter, a demand-side oriented strategy is applied, which focuses on the need for users to learn adequate Internet skills. 


\subsection{Introduction}

From the fact that people are very different it follows that, if we treat them equally, the result must be inequality in their actual position, and that the only way to place them in an equal position would be to treat them differently. (Friedrich August Hayek, 1960, p. 87)

In The Constitution of Liberty, Friedrich August Hayek emphasized that different people require different care to move them to a socially equal level. Putting effort in equalizing social levels in society somehow contrasts with the beliefs of Leo XIII (quoted in Chapter 2, page 22). He argued that differences in skills and preferences make it impossible to move everybody to an equal level. However, this was not seen as a problem; to the contrary, it would benefit economic developments. A balance between the two sides is provided in the capability approach of Sen (1985) in which equal material opportunities go hand in hand with individual freedom of choice. Compensation is offered for inequalities one is not responsible for, but there is also room for personal development and responsibility. Since Internet skills are considered vital assets in the contemporary information society - they affect economic, political, social, health, and cultural domains - and can be considered contributors to social inequality, policies should at least attempt to compensate for low levels of Internet skills, especially for those most in need.

To address different Internet skills levels among the population, it is necessary to use different treatments. This makes Internet skills a complex policy problem that calls for both technological and educational solutions. Here, both solutions are transformed into two broader strategies with the goal of bridging Internet skills divides. The first strategy is supply-side oriented. Here, it is important to improve the usability and quality of the input (i.e., online information and service provision), improving Web sites in such a way that they better cater to users. The other strategy is demand-side oriented and focuses on the need for users to learn adequate Internet skills. The government, producers of hardware and software, institutions with a social and educational mission, and individual citizens and consumers are all responsible for solving the problem of insufficient Internet skills. In this chapter, recommendations for both strategies are suggested. In this way, research Question 10 (How can differences in Internet skills be accounted for?) is addressed. 


\subsection{Improving new media development}

Livingstone (2003) considers literacy to be an emergent property of the interaction and mutual dependence between people and ICT. Therefore, she argues, any communication failure may be as much a result of poor interface design as of poor education. She emphasizes that in discussions of literacy and, especially, of the population's failure to achieve certain levels of literacy, it is implicitly assumed that interfaces are well designed and that the resources are clearly available and merely awaiting appropriate use. When considering new media development, two aspects should be accounted for:

- The interfaces (e.g., Web site designs) that, on the Internet, often leave much room for improvement. Although we do not know how interfaces with poor usability influence Internet skills, it might be expected that people with low levels of operational and especially formal Internet skills have additional difficulty when using these Web sites. After all, formal skills are the skills needed to handle different Web site designs.

- The provided content that should be understandable and suitable for the public for which it is intended.

\subsubsection{Improving interface development}

It is important to realize that many people, especially those with lower levels of educational attainment and seniors, possess low levels of operational and formal Internet skills. Since Internet users that do not belong to the digital generation perform better on both information and strategic Internet skills, they should benefit most from Web sites that account for low levels of operational and formal Internet skills. However, developers and organizations rarely account for users that possess low levels of operational and formal Internet skills when designing and implementing Web sites. Increasingly, information and services are offered online with the expectation that everybody is able to use them. Take, for example, the Dutch Tax and Customs Administration that strongly stimulates people to perform their tax declarations electronically. Although force (by closing traditional channels) might result in actual usage of these services, the people that are unable to use them are somehow left to fend for themselves. Downloading the program needed for the online tax declaration-one of the assignments in the first performance test discussed in Chapter 4-appears to be problematic, especially 
for those over 55 and with low levels of educational attainment. It can be expected that installing and using the program is probably even harder for this group of people. Furthermore, the same segments of the population also experience problems with saving online brochures offered by many institutions.

A useful recommendation that can be derived from the operational Internet skill problems that users experience is to offer clear feedback on Web sites. Feedback that indicates whether a search engine is still searching or feedback that indicates that no results have been found may seem redundant, but is certainly not for people that become confused when they have no clue as to what the Web site is doing or fear that they are doing something wrong. Furthermore, the options that people face on a Web site should be made more explicit, and users should be given a clear and accurate overview of the Web site they are visiting. This also applies to the provided menus that, in many cases, require a better explanation of what each link contains. Detailed instructions on how to use the site would benefit many users. This might sound exaggerated from a designer perspective, but both people with low levels of educational attainment and seniors would benefit. Not everyone knows that search queries do not belong in the address bar or that they should be typed without spaces.

In Chapter 4, several Internet skill-related problems that users experienced were discussed. The analyses revealed that many public Web sites are organized and structured in such a way that they become less accessible and more difficult to use than needed, even considering the complexity of the information and services offered. This also holds for the excessive number of health-related Web sites offered on the Web. From a government as a whole, one might ask whether it is necessary that all organizations maintain their own image and profile when they develop and implement Web sites. Now, interaction between these organizations and users is different for every single Web site that employs its own design and structure. Consistency in Web sites offered would benefit both seniors and lower educated Internet users. Now, they have to learn things anew with every Web site. Furthermore, most of these Web sites have not been tested in a usability lab, since they are considered usable by the developers that mainly focus on providing an attractive Web site. However, only following guidelines for development is insufficient. Unfortunately, from a user-centered perspective, these Web sites leave much room for improvement. From the qualitative analysis, we were able to conclude that users, especially those with low levels of educational attainment and 
those above 55, have to engage in more or less successful learning processes on every Web site again. It might have been more logical to use the same Web site structure and design for every government organization (which would even result in a reduction in costs). After all, civilians consider all these organizations part of the "government." The most important goal should be making the Internet usable as a means of simple information retrieval and service supply. A minimum requirement for both governments and health care organizations when developing Web sites should be the involvement of users that are most likely to experience problems. Furthermore, it would be very helpful for many users, especially seniors and the lower educated, to be informed on exactly where they are on a Web site.

When public and health agencies employ Web sites that are equal in design and structure, the more advanced Web sites that are already present on the Internet could be used as supplementary for those who need the more advanced information and services. This actually implies that organizations should start employing two different Web sites or two branches of sites from a single portal. The more advanced version accounts for the needs of the Internet users with relatively difficult questions and high levels of skills, and the simple version accounts for the needs of the Internet users with relatively simple questions and lower levels of skills. In the relatively simple version, "exaggerated" explanations about the Web site's operation should be provided accompanied by a list of all actions that users can take (e.g., how to proceed to the more advanced Web site, among others). Considering design and didactic approach, the relatively simple version should be equal for every (semi-)government agency. It is important to provide a recognizable, identical, and simple design that leaves out options that are hardly being used. An identical Web site for different organizations both saves on developing costs and caters to the less operational and formal skilled users that are traditionally exposed to new challenges on every single Web site. The easy variants could all be linked in a network of government or health Web sites together covering all basic information and transaction needs. Although this might seem a ludicrous revolutionary idea for many agencies and Web site developers, a complete restructuring of online government and health information provision could prove to be very helpful for users with lower levels of Internet skills. Currently, the fragmented and supply-side oriented nature of the information and service provision of government and health departments that insufficiently cooperate strongly reinforces the inaccessibility of information and the extent of the Internet skills divide. 
A suitable aid for designing government and health-related Web sites (in fact, for all Web sites) is strict guidelines for developers. Although the government does provide such a guideline, the indicators used are shallow. Usually, a more supplyside orientation is taken instead of one accounting for the needs and capabilities of users. When Web sites are tested and compared in both international and national benchmarks, the same shallow indicators are used for ranking sites. The result is that Web sites that offer many information and advanced services tend to score high on these benchmarks. However, the quality of the Web site in terms of accessibility and usability remains unconsidered. This became quite apparent in the first performance test that, for one assignment, used a municipal Web site that ranked number one in the main national public benchmark for public Web sites in 2007. Many subjects from all demographic categories experienced severe usabilityrelated problems (e.g., not being able to use the far too slow rollover menu). Although in scientific literature, several guidelines are proposed for developing citizen-centered Web site designs (e.g., Wang, Bretschneider, \& Gant, 2005), they are hardly being employed by developers of public Web sites. From a usability point of view, the division of public and health Web sites is far from consistent. These guidelines should be stricter and account for segments of the population with low levels of operational and formal Internet skills. Designers should know and bear in mind the characteristics of the target users, their goals, and the strategies they tend to adopt. Unfortunately, the main advantage that national benchmarks seem to achieve is the development of Web sites in terms of advanced and flashy features, not in terms of simplicity and improvements from the user's point of view.

\subsubsection{Improving online information provision}

Since the level of information and strategic Internet skills appeared to be quite low among the general Internet user population, improving online information provision would benefit all users, especially those with lower levels of education. Search processes in general take too many steps and too much time. Although a lack of content-related Internet skills is a major cause, insufficient anticipation of low levels of these skills from a developer point of view worsens the problem. This problem can be addressed by following the staged search process proposed in Chapter 3. 
- Choosing a source of information. Web site developers should accept the fact that Internet users choose their own search engines and adjust their methods of information supply accordingly. Both governments and health institutions attempt to improve the accessibility and connectivity of their information provision by offering large-scale reference Web portals. Although this may be well intentioned, offering portals that attempt to create order in the chaos of government and health Web sites often fails to make things easier for its users. Usually, the portal's scope of information and menu designs are too broad. Furthermore, it is often not clear who offers the reference portal and what the exact purpose is. The excessive amount of information offered often makes the relevant sources harder to find for many users, as was observed in the conducted performance tests. When the subjects were allowed to choose the method of finding the information themselves, they passed government and health portals and turned to Google. A recommendation that follows from this observation is that it is important to monitor where people first arrive on a Web site. These pages should then be supported as entry points. Both government and health institutions should carefully consider the added value of a portal before starting the development. Portals should clearly state for what purposes they are made, who offers them, and what people might expect. The principle of offering a Web portal seems positive, but it is important to offer extra value, something that, in most cases, does not happen. Of course, search facilities should be placed in a noticeable location, as some users prefer to search than to browse. Furthermore, the main search topics should be included in the first few lines of text on the home page.

- Formulating search queries. Many of the subjects in the performance tests experienced problems formulating a reasonable search query. This suggests that it is necessary to develop tools that support the queryformulation process. Lately, Google offers such a support tool by suggesting queries when words are typed into the search bar. Furthermore, users who misspell words should be provided with the right spelling instead of a "no result" response. The Rijksvoorlichtingsdienst (Information Service of the Ministry of General Affairs in the Netherlands) Web site, for example, offers a Web portal with complete and accurate public information that seems to be easy to extract. The 
Web portal uses an intelligent search system that makes suggestions when visitors use broad search queries. These suggestions prove very helpful, especially for people that have trouble defining clear search queries and selecting relevant search results.

- Selecting the most relevant information. Many (government- and health-related) Web sites that are listed in the search result pages after conducting a search often do not provide the information people are expecting to find there. Furthermore, similar (government- and health-related) information is available on different Web sites. As long as this information is complete, there is no problem. Unfortunately, this does not seem to be the case. It is recommended that fewer Web sites be offered but that those offered contain specific information following a clear task division.

- Evaluating information. From the performance tests, it can be concluded that virtually no users evaluate online information. Since many seem to believe that everything on the Internet is true, it might be a good idea for official Web sites to start applying quality marks that can only be used if the content is proven valid and of high quality. When users are informed that these marks exist, they might be more aware of the quality of the information found. Evidently, the Web sites' contents should first be carefully tested and continually scrutinized for quality. Healthcare providers should assume the responsibility of ensuring that patients who use the Internet have access to reliable information to help them understand their disease and possible treatments.

Using online information and services to gain strategic benefits (e.g., choosing a political party based on online information, choosing a health institution with specific needs, or booking the best value city-trip) only seems possible for a minority of the population. Taking into account low levels of strategic Internet skills is a difficult challenge for Web site developers. A reasonable suggestion is to employ more decision support software, programmed with information about actual behavior in decision making. Decision support software such as that used in intelligent search systems or in electronic voting guides appears to be very helpful for a large number of users. Such systems and guides can also be developed for other choices that civilians often face. 


\subsection{Improving Internet skills}

The second strategy to bridge the Internet skills divide focuses on the learning of Internet skills. Here, it is important to account for the whole range of the proposed Internet skills. Only addressing operational and formal Internet skills would ignore important content-related problems that users experience on the Internet. After all, the information and strategic Internet skills levels appear to be most problematic. These skills become increasingly important due to the everexpanding amount of available information. However, this does not mean operational and formal Internet skills can simply be neglected. Segments of the population that have limited operational and formal Internet skills are less likely to employ information and strategic Internet skills, as seems to be the case with seniors.

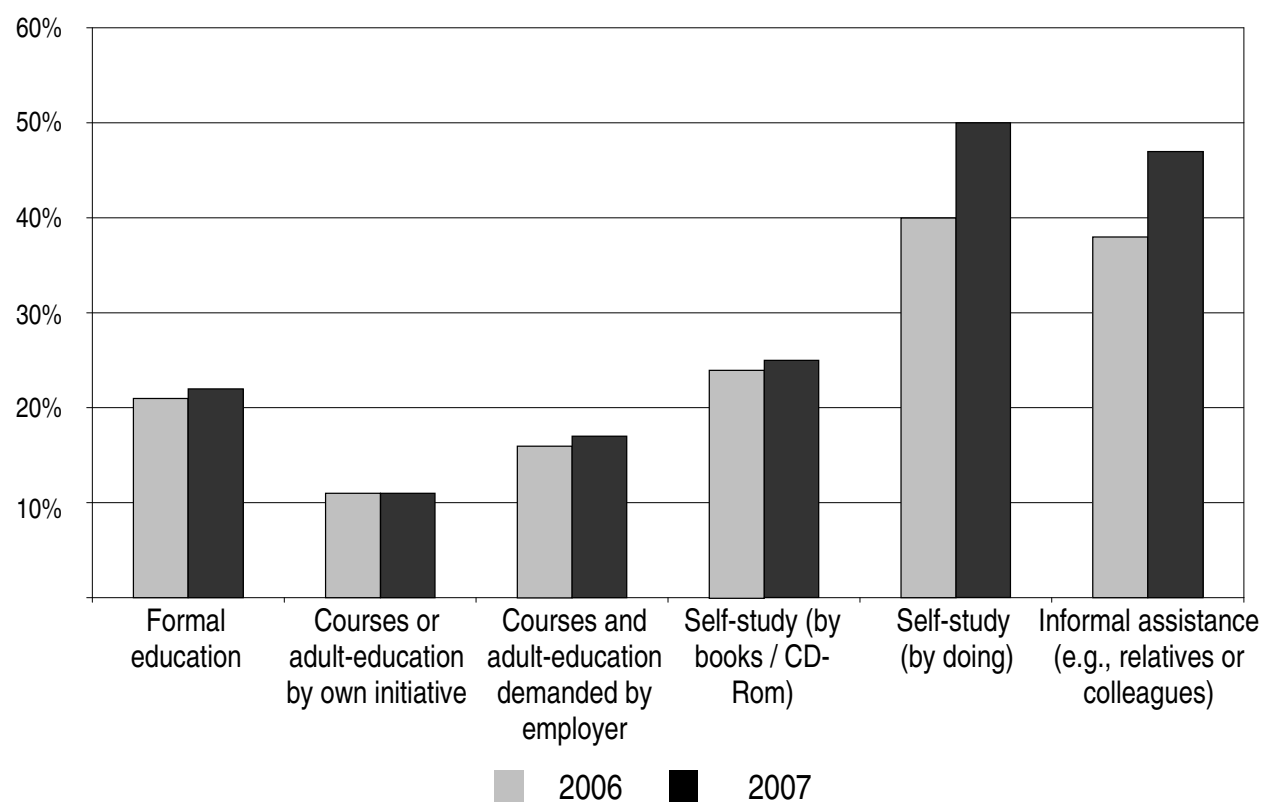

Figure 8.1 Ways of Acquiring Computer and Internet Skills (\% of individuals) Source: Eurostat Community Survey on ICT Usage by Households and by Individuals between 16-74 Years Old (2006-2007). EU27 without Malta. 
There are several options for learning Internet skills. From Figure 8.1, it appears that self-study and informal assistance are the most common ways of learning Internet skills for individuals in Europe. However, as will be argued in the next sections, formal education and specific courses seem more appropriate for segments of the population that suffer from low levels of any of the four Internet skills. This suggests that besides individuals themselves, other parties should assume responsibility for improving the Internet skills levels of the general population. Besides the government, the hardware and software industry, societal organizations of citizens (e.g. organizations for seniors and for the disabled), and employers and employees also have some responsibility for improving the skills that are required in the contemporary information society. The recommendations provided in this section are sorted over life events instead of over the four different Internet skills levels. After all, the results of the performance tests revealed that age and education are the most important factors when identifying Internet skills level. This section starts with upbringing and education. Then, the skills learned during work are addressed, followed by adult education for people that never used the Internet in work or education. Finally, several recommendations are mentioned concerning the elderly, the disabled, and immigrants.

\subsubsection{Learning skills in the context of upbringing and education}

Although the three performance tests only included subjects over the age of 18, this section also provides recommendations for children in primary and secondary education. An important observation regarding operational and formal Internet skills of these children is that they mainly seem to learn them in practice, outside the official educational setting (De Haan \& Huysmans, 2002a). Children's "educational use of the Internet mainly occurs outside the school day with little direction, if any, from teachers" (Harkham Semas, 2002, p. 11). It seems that most children and students possess higher levels of these skills than their teachers do. In primary education in the Netherlands, $90 \%$ of teachers use computers, while in secondary education, only 50\% do (Kennisnet ICT op School, 2007). Less than half of the teachers feel confident enough about their ICT skills to actually teach them. However, the fact that children outrun their teachers and learn these skills mainly in practice does not mean operational and formal Internet skills do not 
require further attention. Spontaneous learning outside the classroom mainly results in learning what is needed. It is likely to be partial and insufficient for many purposes, as many important operations, applications, and opportunities are bypassed. The rapid pace of the evolution of information technology also demands children continually improve their skills. These skills indeed also develop through learning by doing, trying things out, sharing one's problems, and seeing what others are doing. However, mastering the Internet and the specific operational and formal skills for particular applications also requires formal training. This becomes even more apparent when differences between children with different backgrounds are examined. Ba, Tally, and Tsikalas (2002) revealed that middle-income parents developed extensive computer and Internet skills through their jobs and schooling and were able to show rich and varied uses. They engaged their children in critical discussion about the Internet. These children appeared to have sufficient leisure time at home to develop their computer and Internet skills. They used their computers for varied purposes, ranging from work to fun and social interaction. In contrast, many of the low-income parents had never touched a computer and were less able to show practices to their children. Their children appeared to have very little leisure time due to their school's extended-day schedule, and they used their computer primarily for schoolwork.

Fortunately, students are increasingly encouraged to use the computer, the Internet, and e-mail (e.g., for doing homework, looking up information for projects, preparing and presenting talks, and co-operative learning and communicating with classmates) (Cox et al., 2004; Somekh et al., 2002). However, attention to these skills in classes themselves is not a standard component. Often, the skills students learn depend on the teacher of who some have a personal interest in the Internet and spent much time on teaching relating skills, while others feel resistance and simply ignore the subject (Bunz, 2004). In primary education, some attention has been paid to learning operational Internet skills, especially the skills needed for word processing, for example. In secondary education, however, many teachers tacitly assume that students know how to use the computer, surf the Web, and communicate via e-mail (Kuhlemeier \& Hemker, 2007). No further attention is given. This generation is often ascribed distinct characteristics that set them apart from previous generations; they are considered optimistic, team-oriented achievers who are talented with technology (Howe \& Strauss, 2000, 2003). In fact, the main assumption in the literature is that young people of the digital native generation possess sophisticated knowledge of 
information technologies and the skills to use them effectively (Bennett, Maton, \& Kervin, 2008). However, before using the Internet in educational programs, tests should indicate whether students possess an adequate level of operational and formal Internet skills. If they do not, instruction should come first.

Boekhorst (2000) concluded that although pupils were adequately trained in the use of technology, they were not trained to become information literate, as the emphasis is on acquiring operational skills instead of acquiring information. His main recommendation was to engage subjects at all levels to become prepared for their role in contemporary society. Information and strategic skills certainly require more attention in education, since these will not automatically be learned in computer and Internet operations. Students clearly lack information skills (Cohen de Lara, 2005; Kuiper, Volman, \& Terwel, 2004, 2005). From the performance tests, it was concluded that both information and strategic Internet skill levels are quite low, especially with the younger generations. In general, the search process took too many steps and too much time. An educational context is probably the best way to learn information and strategic Internet skills for all age groups. The systematic training of information and strategic Internet skills is an urgent task for all levels of education, from primary to higher education. Unfortunately, Internet skills in general and the acquisition of information skills in particular have a minor role in regular education. Table 8.1 shows that Internet skills in general and the acquisition of information skills in particular have a minor role in Dutch classes, especially in secondary classes in which students use the Internet extensively and therefore should have the information and strategic skills to get the most out of their Internet time. This lack of attention contrasts with the fact that learning these Internet skills is much more difficult than learning operational and formal Internet skills.

Table 8.1 Internet Use by Teachers in Primary and Secondary Education in the Netherlands, 2007

\begin{tabular}{lcc}
\hline & $\begin{array}{c}\text { Primary } \\
\text { education }\end{array}$ & $\begin{array}{c}\text { Secondary } \\
\text { education }\end{array}$ \\
\hline $\begin{array}{l}\text { I provide assignments in which students have to search for the answer on } \\
\text { the Internet. }\end{array}$ & $15 \%$ & $9 \%$ \\
I let the student search for actual sources on the Internet. & $18 \%$ & $10 \%$ \\
When a student works on a school project, I inspire him or her to use the & $32 \%$ & $29 \%$ \\
Internet. & $29 \%$ & $14 \%$ \\
I teach students to use Internet sources selectively. & $11 \%$ & $9 \%$ \\
In my education, students make assignments involving the Internet and & & \\
email. & & \\
\hline Source: Vier in Balans Monitor (2007, p. 50) &
\end{tabular}

Source: Vier in Balans Monitor (2007, p. 50) 
O'Hanlon (2002) concluded that college administrators must not assume that new students arrive with acceptable Internet research skills simply because they say they are competent. O'Hanlon recommended that college administrators assess entry-level skills and provide appropriate instruction in a variety of modes: in research skills courses, short seminars, and workshops, as well as integrated into required courses for various degree programs. Although students might be interested in information Internet skills, they will not take the initiative to learn these themselves (McEuen, 2001). Schools need to present the challenges and create the opportunities or requirements.

We believe that when the Internet is to be used for particular substantial purposes, this is probably best learned by providing special training of information and strategic skills in the contexts engaged (such as particular subjects at school and organizational, hobby, social, and citizen activities). Special attention should be paid to the information Internet skills. It seems that broad standards tend to be provided (for example, by the ACRL) rather than concrete formulations for practical need. The primary objective should be using search engines. Special training in information skills on the Internet is highly recommended for teachers. In addition, it is important to develop educational material that is designed for Internet use. For teaching operational and formal Internet skills, designing separate classes would be best. However, the learning of information and strategic Internet skills might better be implemented in existing courses of the current school curriculum. When information and strategic Internet skills are implemented in existing courses such as language, history, biology, and geography, they will be more effectively picked up than when special computer classes are developed. Furthermore, teachers will be more motivated to spend additional time and effort.

Information and strategic Internet skills can also be added to so-called media education. This part of the curriculum often primarily discusses knowledge derived from the mass media and effects of the media. Considering the Internet, much of the attention goes to the dangers or risks for (mainly) young people on the Internet and how to deal with them. For example, children and students need information and strategic Internet skills to learn about the risks of privacy intrusions in their daily Internet activities and to prevent the naiveté they frequently demonstrate in these matters. Teachers should also better understand how information is presented on the Internet and should be able to teach new 
concepts and approaches to help students comprehend and discriminate the content validity and reliability of information available on the Internet.

Finally, as can be derived from the prior recommendations, it might be necessary to change current educational systems. Regarding technology in education, students have been variously described as disappointed (Oblinger, 2003), dissatisfied (Levin \& Arafeh, 2002), and disengaged (Prensky, 2005).

\subsubsection{Learning skills in the context of labor}

Another social sphere in which people can learn Internet skills systematically is the labor context. Middle-aged people that did not get the chance to acquire Internet skills in education actually depend on their work or on adult education to learn these skills. It appears that approximately $37 \%$ of the Dutch population between 18 and 65 follows a computer and/or an Internet course, most of them within the scope of work (Van Damme, De Haan, Kraan, et al., 2005). Unfortunately, these courses mainly address word processing (Van Damme et al., 2005). Classes paid for by employers often have a very specific character that primarily focuses on the ability to perform the accompanying goals of the job. This especially goes for the more complex applications. The four Internet skills defined in Chapter 3 are rarely an objective of teaching. However, they are also appropriate and sometimes even necessary for employees. In contemporary society, the number of informationrelated jobs continues to increase. This makes proper search queries, sharp selections, and an appreciation of valid information sources very important, especially since information Internet skills have a very functional purpose in information jobs. A lack of these skills might even result in a serious drop in productivity. Strategic Internet skills at work include the processing and use of information for the specific position one has and for the overall company's goal, varying from sales and recruitment to the development of organizational strategies.

Table 8.2 shows how employees learn digital skills. Although the precise skills are not specified, they also include Internet skills. Unfortunately, comparable data for Internet skills only are not available. In general, $40 \%$ of the employees have gained digital skills from courses. It appears, however, that learning digital skills from colleagues is even more common. This is done by $42 \%$ of the employees. Forty 
percent have taught themselves digital skills. It remains unclear what the relative contribution of every source is to the actual level of digital skills. There are, however, some indications for the actual level of digital skills that result from courses. The Dutch employees themselves indicate that they are very happy with their own level of digital skills. In 2002, they scored a 7.5 on a 10-point scale when assessing this level themselves. It appeared that $19 \%$ of the employers even scored a nine or ten. Only 7\% scored an unsatisfactory mark (AIAS/EUR, 2002). The findings from the conducted performance tests, however, suggest that employers and employees do not score better than inactive or unemployed civilians do. A comparable performance test conducted among 98 civil servants (with the same assignments as used in the performance tests among the Dutch population) revealed that these servants do not differ from the general user population in their Internet skills (Van Deursen \& Van Dijk, 2010). Furthermore, in Chapter 4, it was concluded that when assessing their own level of Internet skills, subjects primarily have operational Internet skills in mind. This might suggest that employees' high level of digital skills probably means that they are very capable in handling the functional applications they have to use at work. It is very likely they overrate their Internet skills levels, especially their information and strategic Internet skills. This would imply some serious problems. The $40 \%$ of employees that obtain their digital skills at work mainly learn operational skills taught at courses for basic applications or very special programs (Van Damme et al., 2005). Only a very limited number of formal navigation and orientation skills are learned. If any attention is paid to information and strategic Internet skills, then it only involves utilizing a specific application. General information and strategic Internet skills, even the proper use of a search system, are not considered.

Table 8.2 Ways of Acquiring Digital Skills at Work in the Netherlands, $\%$ of employees

\begin{tabular}{lcccccc}
\hline & $\begin{array}{c}\text { High } \\
\text { education }\end{array}$ & $\begin{array}{c}\text { Medium } \\
\text { education }\end{array}$ & $\begin{array}{c}\text { Low } \\
\text { education }\end{array}$ & $\begin{array}{c}\text { Basic } \\
\text { skills }\end{array}$ & $\begin{array}{c}\text { Complex } \\
\text { skills }\end{array}$ & Total \\
\hline Learned at school & 13 & 8 & 1 & 7 & 9 & 8 \\
Learned at a course & 37 & 43 & 38 & 39 & 46 & 40 \\
Learned from a & 33 & 46 & 49 & 42 & 39 & 42 \\
colleague & 4 & 10 & 17 & 9 & 9 & 10 \\
Learned from a superior & 41 & 34 & 34 & 41 & 39 & 40 \\
Learned myself & 51 & & & & &
\end{tabular}

Source: AIAS/EUR (CentER panel data University of Tilburg), 2002.

Just as teachers more or less assume that students learn their Internet skills at home or in practice, employers expect their employees to have already learned sufficient levels of Internet skills at home or at school. It seems that, in their 
opinion, employees need training for specific applications at most. Especially for the highly educated, it is assumed that they possess all levels of Internet skills. These skills are rarely asked for, let alone tested. Considering the low levels of information and strategic Internet skills that were observed in the three performance tests, one might expect that the Internet is used insufficiently at work. Employees experience problems in using search systems similar to those the average civilian does. The consequence might be a lack of productivity or insufficient support for innovation. It is recommended to test the level of all four Internet skills when hiring new employees. ICT-related courses and training should focus more heavily on the Internet. Proper use of the Internet will improve productivity, especially in information-related professions. In addition, even more straightforward media education could be added, involving safe use of the Internet, privacy protection, intellectual property rights, and other elementary juridical Internet problems.

\subsubsection{Learning skills in the context of adult education}

Table 8.3 Sources of Internet Skills in the Netherlands, $\%$ of persons in three age groups

\begin{tabular}{lccc}
\hline & $\mathbf{1 8 - 3 4}$ & $\mathbf{3 5 - 4 9}$ & Over $\mathbf{5 0}$ \\
\hline Self study & 92 & 87 & 64 \\
Computer courses & 32 & 46 & 48 \\
Computer books & 41 & 46 & 51 \\
Secondary education & 25 & 1 & 0 \\
Higher education & 56 & 17 & 6 \\
Colleagues & 54 & 56 & 43 \\
Friends, acquaintances, and neighbors & 52 & 41 & 24 \\
Parents & 20 & 1 & 0 \\
Children & 3 & 18 & 29 \\
\hline
\end{tabular}

Source: Van Dijk, De Haan, \& Rijken, 2000.

People above 35 that do not learn Internet skills in education or at work increasingly depend on the social environment and their own initiatives. Table 8.3 reveals that this should not be a major problem. For all ages, it appears that private study is the most important source for obtaining Internet skills. However, for older people and those with lower levels of education, private study seems less of an option. For seniors and people of middle age, books and courses gain importance. Seniors lose support from colleagues when they retire. They have less of a social network to fall back on in terms of computers and the Internet. People 
over 50 instead turn relatively often to their children. For older and lower educated adults, a structured approach involving courses and specialized material is no luxury. In the Netherlands, adult education does not meet these demands sufficiently. The major part of computer and Internet courses is initialized and paid for by employers.

Another option is to follow courses on one's own initiative and to pay for it one's self (e.g., the European Computer Driving License of which 16,000 modules were sold in the Netherlands in 2007). Although they are becoming scarce, community centers also offer computer and Internet courses. In the Netherlands, at the beginning of the current millennium, several so-called "digital playgrounds" in neighborhood centers were opened, intended to teach computer and Internet use. The playgrounds were quite popular, mostly among seniors, the lower educated, and immigrants. Over the years, these facilities became rundown or were taken over by institutional initiatives like public libraries, regular community centers, or even nursing homes for the elderly. This often happened because of the assumption that the problem of lack of skills was almost solved since everybody nowadays has access to computers and the Internet at home.

From the results presented in Chapter 4, it appears that the Internet skills problem has been far from resolved. The elderly and lower educated people especially appear to have low levels of operational and formal Internet skills. The majority of the population possesses insufficient information and strategic Internet skills. Initiatives for people that cannot turn to courses at work or education are more than welcome. Community centers, for example, can integrate all four Internet skills in the basic courses that were originally developed for the digital playgrounds in existing community facilities. This integration should take a perspective that fits the daily needs of the stakeholder, namely seniors, lower educated people, and immigrants. This would motivate and support the learning of these skills (Van Dijk, 2005; Warschauwer, 2003). Public libraries seem an appropriate venue to train adults in the improvement of information Internet skills, at least when calling in a professional staff in addition to simply installing the necessary equipment. In general, all public and private institutions of adult education should receive more means and a competent staff to meet the needs of Internet courses. People should be able to participate in these courses at low cost. The same goes for elementary computer and Internet instruction in the context of education and citizen programs for immigrants. 


\section{Case: Internet Boot Camp}

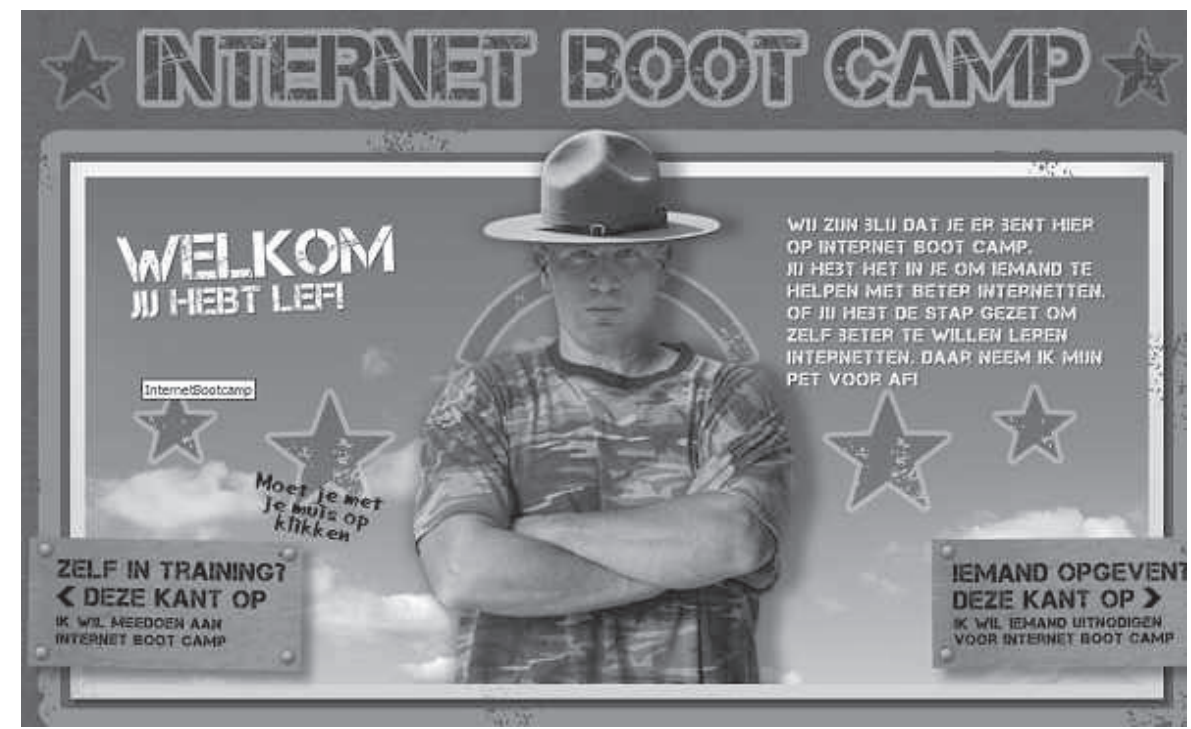

Screenshot of the Web site Internet Boot Camp

On November 20, 2009, the Assistant secretary of the Ministry of Economic Affairs kicked of Internet Boot Camp (www.internetbootcamp.nl). This Web site and the accompanying campaign is part of the program Digivaardig \& Digibewust (Digi-skilled and Digi-aware) which aims at decreasing the number of computer and Internet illiterates in the Netherlands. The goal of the Web site is to assist people that feel uncomfortable in using the Internet. The Web site is freely accessible and is used in courses and workshops offered by libraries.

The contents of the Web site are developed by Alexander van Deursen and Jan van Dijk. The Web site contains four modules, one for testing operational Internet skills, one for formal Internet skills, one for information Internet skills, and one for strategic Internet skills. The Web site is unique in the fact that skills are tested by providing actual assignments to be completed on the Internet. The assignments used in the performance tests discussed in this dissertation were used for input. Since the main goal of the Web site was to improve one's level of Internet skills, providing useful feedback to the users based on their assignment completion was essential. Most challenging was accounting for all the possible Internet skills-related problems that users can experience during the assignment completion. This had to be recognized by the Web site's software. Eventually, based on the encountered problems, specific recommendations were composed and presented (online and by e-mail) to the user after finishing a module. 


\subsubsection{Learning skills in the context of people with specific needs}

Internet skills are highly dependent on both education and age. From this point of view, several groups with special needs can be defined. First, individuals above 55 and the lower educated have both gained some attention in the prior section. Furthermore, there are groups with specific problems: illiterates, the disabled, and immigrants. From the studies discussed in this dissertation, it is not possible to draw specific conclusions for these groups, as they were underrepresented in the performance tests. However, some recommendations can be provided from other sources of knowledge.

Seniors seem to be mainly hindered by their lack of operational and formal Internet skills. A remarkable conclusion was that they would score better than the younger generations on the content-related Internet skills when they were not limited by their medium-related Internet skills. This means that when they get support in Internet courses that are adapted to their speed, style of learning, and physical limitations, they should be able to cross the threshold of the online information environment. However, this does not mean that they will favor the Internet over traditional channels like telephones and counter desks, as the need for social contact and reassurance remains. The elderly need specific training or personal assistance for improving their operational and formal Internet skill levels. In 2008, the Dutch Statistical Bureau (CBS) reported that seniors only use the most relevant Internet applications like e-mail and elementary information searches, probably because they lack operational and formal Internet skills.

Table 8.4 Actual and Desired Sources for Acquiring Internet Skills in the Netherlands, $\%$ of Seniors over 55

\begin{tabular}{lcc}
\hline & Actual source & Desired source \\
\hline Self study & 54 & 28 \\
Course & 18 & 39 \\
$\quad$ Specific for seniors & & 13 \\
$\quad$ General & & 4 \\
$\quad$ Private & 12 & \\
At work & 9 & 8 \\
Children & 7 & 8 \\
Family, friends, and neighbors & & \\
Other & &
\end{tabular}


In a study conducted by the Dutch Social Cultural Planning Office (Van Ingen, De Haan, \& Duimel, 2007)), seniors were asked for their sources of Internet skills training. The results are presented in Table 8.4. The table reveals that the social network that offers support when using the Internet is not really their desired source from which to gain help. They probably feel unconfident or even ashamed to ask for help. The table also reveals that self-study seems inadequate. Fifty-six percent of the seniors indicated that they would like to learn Internet skills in formal courses, preferably aimed at seniors. Regular courses might be too fast for seniors and do not specifically address the problems seniors face. They need Internet courses that are adapted to their speed and style of learning and to their physical limitations that increase with age. Special courses can be offered by commercial organizations and voluntary organizations. According to Wales (2004, p. 41), for older people "who are usually considered more frail or vulnerable," the Internet is more like "a person issue before a technology issue." Wales suggests that seniors need a personal approach, because many older people do not see the benefits of IT and might have physical limitations. Seniors who participate in programs in which youth and young adults teach computer skills to older adults show a positive change in attitudes towards computers and the Internet and gain confidence in their own proficiency with technology (Kolodinsky, Cranwell, \& Rowe, 2002). Youth who participate in these programs as the "teacher" show improved leadership skills and gain a sense of personal-worth based on their contributions to society (Kolodinsky et al., 2002). This personal approach can, for example, also be provided by healthcare providers. By proactively helping patients to find health-related information on the Internet, it is ensured that these patients will be directed to comprehensive sites.

Within the lower-educated part of the population, not only seniors have low levels of operational and formal Internet skills. In fact, all lower-educated civilians seem to benefit from training in operational and formal Internet skills. A much larger problem for this group, however, is the lack of information and strategic Internet skills. Besides general education, it seems that only adult-education can provide assistance here. For the specific group of illiterates, the use of computers and the Internet seems almost impossible, since they largely require the ability to read and write. However, special aids such as audiovisual interfaces, multimedia programs, and touch screens can be designed and offered to them. 
Disabled people are twice as likely as non-disabled people to have no educational qualification and are far less likely to have access to the Internet or possess the skills needed to use it (Thoreau, 2006). Organizations for the disabled increasingly call for the development of Web sites accessible for the disabled and for specific adjustments in hard- and software that is needed to use the Internet. The Internet's capacity for text, graphics, animation, video, and audio could also potentially exclude disabled people with visual, hearing, or mobility impairments (Goggin \& Newell, 2003). For the disabled, Web sites should be made more accessible with special aids, and additional services might be provided if necessary (e.g., homecare). Furthermore, voluntary organizations of/for disabled people could give Internet classes that are adapted to the specific needs of the disabled.

The final group that might require special attention is immigrants. In the Netherlands, this group is quite diverse. Large-scale survey research revealed that especially Turkish, Moroccan, and non-Western immigrants lag behind most regarding digital skills (D'Haenens, 2003). It is also very likely that there are differences between first- and second-generation level immigrants. The first generation probably overlaps with the age problems discussed, strengthened by illiteracy regarding the Dutch language. However, when adjusting for age and education, the level of digital skills still differs between all generations of natives and immigrants (Van Ingen, De Haan, \& Duimel, 2007). This difference is probably caused by language and cultural factors. For ethnic minorities, the supply of information and services should preferably be designed to provide more multicultural choice options. More training materials can be provided that also account for minority languages. Finally, Web site designs should also be inspired by minority cultural experiences. 

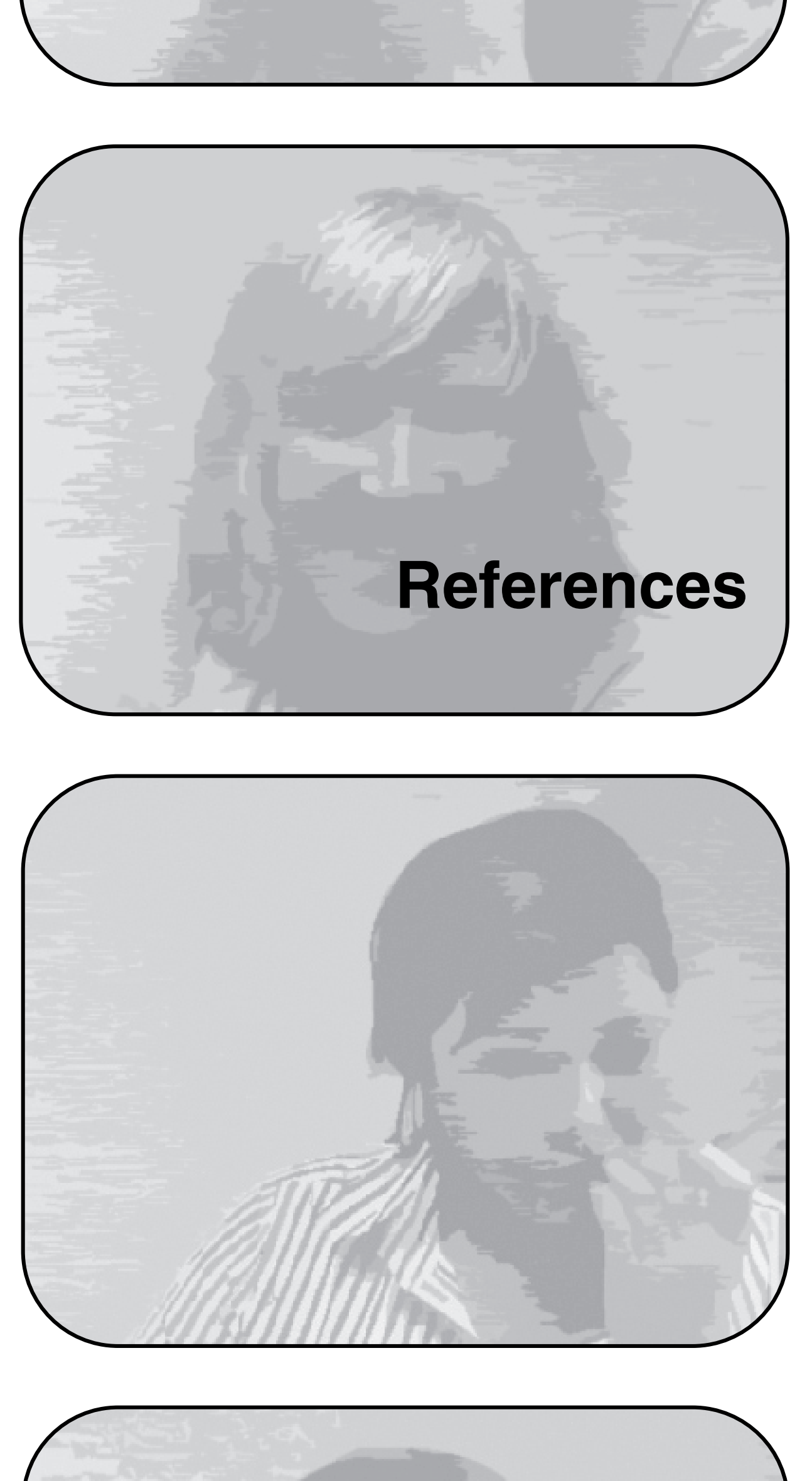
Adams, J.A. (1984). Networked computers promote computer literacy and computer-assisted instruction. Technological Horizons in Education, 11(8), 9599.

Adams, D., \& Hamm, M. (2001). Literacy in a multimedia age. Norwood, MA: Christopher-Gordon.

Ahuja, J.S., \& Webster, J. (2001). Perceived disorientation: an examination of a new measure to assess web design effectiveness. Interacting with Computers, 14(1), 15-29.

American Library Association (1989). Presidential Committee on Information Literacy. Final report. Chicago, IL: ALA.

Anandarajan, M., Simmers, C., \& Igbaria, M. (2000). An exploratory investigation of the antecedents and impact of internet usage: an individual perspective. Behaviour \& information technology, 19(1), 69-85.

Anttiroiko, A.V., Lintilä, L., \& Savolainen, R. (2001). Information society competencies of managers: conceptual considerations. In E. Pantzar, R. Savolainen, \& P. Tynjälä (Eds.), In search for a buman-centered information society. (pp. 27-57). Tampere: Tampere University Press.

Armstrong, S., \& Warlick, D. (2004). The new literacy. TechLearning, 25(2), 24-31.

Attewell, P. (2001). The First and Second Digital Divides. Sociology of Education, 74(3), 252-259.

Auferderheide, P. (1993). Media literacy: A report of the national leadership conference on media literacy. Aspen, CO: Aspen Institute.

Aula, A., \& Nordhausen, K. (2006). Modeling successful performance in Web searching. Journal of the American Society for Information Science and Technology, 57(12), 1678-93.

Ba, H., Tally, W., \& Tsikalas, K. (2002). Investigating children's emerging digital literacies. Journal of Technology, Learning and Assessment, 1(4), 1-48.

Balasubramanian, V. (1994). State of the art review on bypermedia issues and applications. Retrieved from: http://www.isg.sfu.ca/ duchier/misc/ hypertext_review/index.html

Bawden, D. (2001). Information and digital literacies: A review of concepts. Journal of Documentation, 57(2), 218-259.

Beitzel, S.M., Jensen, E.C., Chowdhury, A., Grossman, D., \& Frieder, O. (2004). Hourly analysis of a very large topically categorized Web query log. In P.D. Bruza \& J. Thom (Eds.), Proceedings of the 27th annual international conference on Research and development in information retrieval (pp. 321-328). Sheffield, UK: SIGIS. 
Benigeri, M., \& Pluye, P. (2003). Shortcomings of health information on the Internet. Health Promotion International, 18(4), 381-386.

Bennett, S., Maton, K., \& Kervin, L. (2008). The digital natives debate: A critical review of the evidence. Britsh Journal of Educational Technology, 39(5), 775786.

Bentler, P.M. (1989). Theory and implication of EQS: A structural equations program. Los Angeles, CA: BMDP Statistical Software.

Berners-Lee, T., \& Fischetti, M. (2000). Weaving the Web: The original design and ultimate destiny of the World Wide Web. New York, NY: HarperCollins.

Bikson, T.K., \& Panis, C.W.A. (1997). Computers and connectivity: Current trends. In S. Kielser (Ed.), Culture of the Internet (pp. 407-430). Mahwah, NJ: Lawrence Erlbaum Associates.

Bilal, D. (2002). Children's use of the Yahooligans! Web search engine. III. Cognitive and physical behaviors on fully self-generated tasks. Journal of the American Society for Information Science and Technology, 53(13), 1170-83.

Bilton, T., Bonnett, K., Jones, P., et al. (2002). Introductory Sociology. London: Palgrave Macmillan.

Bimber, B. (2000). The gender gap on the Internet. Social Science Quarterly, 81(3), 868-76.

Bimber, B. (2003). Information and American Democracy. New York, NY: Cambridge University Press.

Birru, M.S., Monaco, V.M., Charles, L., et al. (2004). Internet usage by low-literacy adults seeking health information: an observational analysis. Journal of Medical Internet Research, 6(3), e25.

Boekhorst, A.K. (2000). Informatievaardig worden in het onderwijs, een informatiewetenschappelijk perspectief: een vergelijkende gevallenstudie in Nederland en ZuidAfrika. Pretoria: Universiteit van Pretoria.

Boekhorst, A.K. (2003). Information literacy in the Netherlands. On becoming information literate in the Netherlands. In C. Basisli (Ed.), Information Literacy in Europe: a first insight into the state of the art of Information Literacy in the European Union (pp. 187-205). Roma: Consiglio Nazionale delle Ricerche.

Bonfadelli, H. (2002). The Internet and knowledge gaps: a theoretical and empirical investigation. European Journal of Communication, 17(1), 65-84. 
Boomershine, T. (1987). Peter's denial as polemic or confession: the implications of media theory for biblical hermeneutics. In L.H. Silberman (Ed.), Orality, aurality and Biblical narrative (pp.47-68). Decatur, GA: Scholars Press.

Bourdieu, P. (1984). Distinction: a social critique of the judgment of taste. London: Routledge.

Boyd, D.A., \& Bee, H. (2009). Lifespan development. Boston: Pearson.

Boyd, D.M., \& Ellison, N.B. (2007). Social network sites: Definition, history, and scholarship. Journal of Computer-Mediated Communication, 13(1), 210-230.

Brand-Gruwel, S., Wopereis, I., \& Vermetten, Y. (2005). Information problem solving by experts and novices: Analysis of a complex cognitive skill. Computers in Human Behavior, 21(3), 487-508.

Breivik, P.S. (2001). Information literacy. Retrieved from http://www.nordinfo. helsinki.fi/nordnytt/nnytt4_01/breivik.htm

Briggs, C.F. (2000). Literacy, reading, and writing in the medieval West. Journal of Medieval History, 26(4), 397-420.

Brown, J.A. (1998). Media literacy perspectives. Journal of communication, 48(1), 4457.

Browne, M.W., \& Cudeck, R. (1993). Alternative ways of assessing model fit. In K.A. Bollen, \& J.S. Long (Eds.), Testing structural equation models (pp. 136162). Newbury Park, CA: Sage.

Brundidge, J., \& Rice, R.E. (2008). Political engagement online: Do the information rich get richer and the like-minded become more similar? In A. Chadwick, \& P.N. Howard (Eds.), The handbook of Internet politics (pp. 144-156). London and New York: Routledge.

Buckingham, D. (2007). Beyond technology: Children's learning in the age of digital culture. Cambridge: Polity.

Bucy, E., \& Newhagen, J. (2004). Media access: Social and psychological dimensions of new technology use. London: LEA.

Buente, W., \& Robbin, A. (2008). Trends in internet information behavior, 20002004. Journal of the American Society for Information Science and Technology, 59(1), 1743-60.

Bundy, A. (2004). One essential direction: information literacy, information technology fluency. Journal of eLiteracy, 1(1), 7-22.

Bunz, U. (2004). The computer-Email-Web (CEW) Fluency Scale - Development and validation. International journal of human-computer interaction, 17(4), 479506. 
Bunz, U. (2009). A generational comparison of gender, computer anxiety, and computer-email-web fluency. Studies in media \& information literacy education, 9(2), 54-69.

Cahoon, B. (1998). Teaching and learning Internet skills. New Directions for Adult and Continuing Education, 78, 5-13.

Carvin, A. (2000). More than just access: Fitting literacy and content into the digital divide equation. Retrieved from http://net.educause.edu/ir/library/pdf/ ERM0063.pdf

Castells, M. (1996). The Rise of the Network Society. Blackwell: Oxford.

Castells, M. (1999). Flows, networks and identity: A critical theory of the international society. In M. Castells, et al. (Eds.), Critical Education in the New Information Age. Rowman and Littlefield: Lanham.

Castells, M. (2004). The network society: a cross-cultural perspective. Northampton, MA: Edward Elgar.

Chadwick-Dias, A., McNulty, M., \& Tullis, T. (2003). Web usability and age: how design changes can improve performance. In M. Zajicek \& A. Edwards (Eds.) Proceedings of the 2003 conference on Universal usability (pp. 30-37), New York, NY: ACM.

Cho, J., Gil de Zúñiga, H., Rojas, H., \& Shah, D. (2003). Beyond access: The Digital Divide and Internet Uses and Gratifications. IT\&Society, 1(4), 4672.

Choo, C.W., Detlor, B., \& Turnbull, D. (2000). Web Work: Information seeking and knowledge work on the World Wide Web. Kluwer Academic Publishers, Dordrecht.

Clark, W. (2001). Kids and teens on the Net. Canadian Social Trends, 62, 6-10.

Cody, M.J., Dunn, D., Hoppin, S., \& Wendt, P. (1999). Silver surfers: Training and evaluating Internet use among older adult learners. Communication Education, 48(4), 269-286.

Cohen, J. (1988). Statistical power analysis for the behavioral sciences. Hillsdale, NJ: Erlbaum.

Cohen de Lara, H. (2005). Hoe word ik webwijs. Didaktief, 8(35), 22-24.

Compaine, B.M. (2001). The Digital Divide: Facing a Crisis or Creating a Myth? London: MIT Press.

Conklin, L. (1987). Hypertext: An introduction and survey. Computer, 20(9), 17-41.

Connelly, S.L., Hasher, L., \& Zacks, R.T. (1991). Age and reading: the impact of distraction. Psychology and Aging, 6(4), 533-541 
Correia, R.A.M., \& Teixeira, J.C. (2003). Information Literacy: an integrated concept for a safer Internet. Online Information Review, 27(5), 311-20.

Cox, M., Webb, M., Abbott, C., Blakeley, B., Beauchamp, T., \& Rhodes, V. (2004). A review of the research literature relating to ICT and attainment. London, Becta.

D’Alessandro, D.M., Kingsley, P., \& Johnson-West, J. (2001). The readability of pediatric patient education materials on the World Wide Web. Archives of Pediatrics and Adolescent Medicine, 155(7), 807-812.

Danielson, D.R. (2003). Transitional Volatility in Web Navigation: Usability Metrics and User Behavior. IT \& Society, 1(3), 131-158.

Davis, P.M. (2003). Effects of the web on undergraduate citation behavior: Guiding student scholarship in a networked age. Libraries and the Academy, 3(1), 41-51.

De Castell, S., \& Luke, A. (1988). Defining "literacy" in North American schools: Social and historical conditions and consequences. In E.R. Kingten, B.M. Kroll, \& M. Rose (Eds.), Perspectives on literacy (pp. 159-174). Carbondale, IL: Southern Illinois University Press.

De Haan, J. (2003). IT and Social Inequality in the Netherlands. IT \& Society, 1(4), 27-45.

De Haan, J. (2004). A multifaceted dynamic model of the digital divide. IT\&Society, 1(7), 66-88.

De Haan, J., \& Huysmans, F. (2002a). Van huis uit digitaal; Verwerving van digitale vaardigheden tussen thuismilieu en school. Den Haag: SCP.

De Haan, J., \& Huysmans, F. (2002b). Digitale vaardigheden in de informatiesamenleving. In J. de Haan, \& F. Huysmans (Eds.), E-cultuur: Een empirische verkenning (pp. 61-73). Den Haag: SCP.

De Haan, J., \& Huysmans, F. (2002c). Differences in time between Internet users and nonusers in the Netherlands. IT\& Society, 1(2), 67-85.

De Haan, J., Huysmans, F., \& Steyaert, J. (2002). At home in a digital world. Acquiring digital skills between home and school. The Hague: SCP.

D'Haenens, L. (2003). ICT in multicultural society. The Netherlands: A context for sound multiform media policy? International Communication Gazette, 65(4-5), 405-421.

De Mul, J. (2005). Filosofie in cyberspace. Reflecties op de informatie- en communicatietechnologie. Kampen: Klement. 
De Vries, B., Van Der Meij, H., \& Lazonder, A.W. (2008). Supporting reflective web searching in elementary schools. Computers in Human Behavior, 24(3), 649-665.

Diaz, J.A., Griffith, R.A., Ng, J.J., Reinert, S.E., Friedmann, P.D., \& Moulton, A.W. Patients' use of the Internet for medical information. Journal of General Internal Medicine, 17(3), 180-185

DiMaggio, P., \& Hargittai, E. (2001). From the 'Digital divide' to 'Digital inequality': Studying internet use as penetration increases. Working Paper Series 15: Princeton University Center for Arts and Cultural Policy Studies.

DiMaggio, P., Hargittai, E., Celeste, C., \& Shafer, S. (2004). From Unequal Access to Differentiated Use: A Literature Review and Agenda for Research on Digital Inequality. In Neckerman, K. (Ed.), Social Inequality (pp. 355-400). New York, NY: Russell Sage Foundation.

DiMaggio, P., \& Bonibowski, B. (2008). Make money surfing the web? The impact of internet use on the earnings of U.S. Workers. American Sociological Review, 73(2), 227-250.

Dinet, J., Favart, M., \& Passerault, J.M. (2004). Searching for information in an Online Public Access Catalogue (OPAC): The impacts of information search expertise on the use of Boolean operators Journal of Computer Assisted Learning, 20(5), 338-346.

Doyle, C.S. (1994). Information literacy in an information society: a concept for the information age. Syracuse, NY: ERIC Clearinghouse.

Dunn, K. (2002). Assessing information literacy skills in the California state university: a progress report. The Journal of Academic Librarianship, 28(1-2), 26-35.

Eastin, M.S., \& LaRose, R. (2000). Internet self-efficacy and the psychology of the digital divide. Journal of Computer-Mediated Communication, 6(1).

Edwards, D., \& Hardman, L. (1989). Lost in hyperspace: Cognitive mapping navigation in a hypertext environment. In R. McAleese (Ed.), Hypertext: Theory into practice (pp 90-105). Norwood, NJ: Ablex Publishing.

Eisenberg, M., \& Berkowitz, R. (1990). Information problem solving: The big six skills approach to library and information skills instruction. Norwood, NJ: Ablex.

Ellis, D. (1989). A behavioural model for information retrieval system design. Journal of Information Science, 15(4-5), 237-247.

Eshet-Alkalai, Y. (2004). Digital Literacy: A conceptual framework for survival skills in the digital era. Journal of Educational Multimedia and Hypermedia, 13(1), 93-106. 
Eshet-Alkalai, Y., \& Amichai-Hamburger, Y. (2004). Experiments in digital literacy. Cyberpsychology and Behavior, 7(4), 421-429.

Eysenbach, G., \& Köhler, C. (2002). How do consumers search for and appraise health information on the World Wide Web? Qualitative study using focus groups, usability tests, and in-depth interviews. British Medical Journal, 324(7337), 573-577.

Eysenbach, G., \& Köhler, C. (2003). What is the prevalence of health-related searches on the World Wide Web? Qualitative and quantitative analysis of search engine queries on the internet. In M. Musen (Ed.), Proceedings of the 2003 AMIA Annual Symposium (pp. 225-9). Washington, DC: AMIA.

Eysenbach, G., Powell, J., Kuss, O., \& Eunryoung, S. (2002). Empirical studies assessing the quality of health information for consumers on the World Wide Web: A systematic review. Journal of American Medical Association, 287(20), 2691-2700.

Fallows, D. (2005). How men and women use the Internet. Washington, DC: Pew Internet and American Life Project.

Fidel, R., Davies, R.K., Douglass, M.H., Holder, J.K., Hopkins, C.J., \& Kushner, E.J. (1999). A visit to the information mall: Web searching behaviour of high school students. Journal of the American Society for Information Science, 50(1), 24-37.

Forman, C. (2005). The Corporate Digital Divide: Determinants of Internet Adoption. Management Science, 51(4), 641-654.

Fornell, C., \& Larcker, F. D. (1981). Evaluating structural equation models with unobservable variables and measurement error. Journal of Marketing Research, 18(1), 39-50.

Fox, S., \& Fallows, D. (2003). Internet health resources. Pew internet \& American life project. Retrieved from http://www.pewinternet.org/pdfs/PIP_Health _Report_July_2003.pdf

Fox, S., \& Rainie, L. (2002). The online bealth care revolution: how the Web helps Americans take better care of themselves. Pew Internet \& American Life Project: online life report.

Freeman, R. (2002). The labour market in the new information economy. Oxford Review of Economic Policy, 18(3), 288-305.

Freese, J., Rivas, S., \& Hargittai, E. (2006). Cognitive ability and internet use among older Adults. Poetics, 34(4), 236-249.

Fuchs, C. (2008). Internet and society: Social Theory in the information age. New York: Routeledge. 
Fuchs, C., \& Horak, E. (2008). Africa and the digital divide. Telematics and Informatics, 25(2), 99-116.

Gilster, P. (1997). Digital literacy. New York: Wiley.

Goggin, G., \& Newell, C. (2003). Digital Disability: The Social Construction of Disability in New Media. Lanham, MD: Rowman \& Littlefield.

Goldin, C., \& Katz, L.F. (2008). The race between education and technology. Cambridge, MA: The Belknap Press of Harvard University.

Golding, P. (1996). World-wide Wedge: Division and contradiction in the global information infrastructure. Monthly Review, 48(3), 70-86.

Golding, P., \& Murdock, G. (2001). Digital divides: communications policy and its contradictions. New Economy, 8(2), 110-115.

Goss, E.P., \& Phillips, J.M. (2002). How Information Technology Affects Wages: Evidence Using Internet Usage As a Proxy for IT Skills. Journal of Labor Research, 23(3), 463-474.

Goulding, A., \& Spacey, R. (2002). Women and the Information Society: Barriers and Participation. Paper presented at 68th IFLA Council and general conference, Glasgow. Retrieved from http://ericit.org/fulltext/IR058567 .pdf

Griffiths, F., Lindenmeyer, A., Powell, J., Lowe, P., \& Thorogood, M. (2006). Why are health care interventions delivered over the Internet? A systematic review of the published literature. Journal of Medical Internet Research, 8(2), e10.

Gwizdka, J., \& Spence, I. (2007). Implicit measures of lostness and success in web navigation. Interacting with Computers, 19(3), 357-369.

Hakkarainen, K., Ilomaki, L., Lipponen, L., et al. (2000). Students' skills and practices of using ICT: results of a national assessment in Finland. Computers \& Education, 34(2), 103-117.

Hargittai, E. (2002). Second-Level Digital Divide: Differences in people's online skills. First Monday, 7(4).

Hargittai, E. (2005). Survey measures of web-oriented digital literacy. Social Science Computer Review, 23(3), 371-379.

Hargittai, E. (2010). Digital $\mathrm{Na}$ (t)ives? Variation in Internet skills and Uses among members of the "Net generation." Sociological Inquiry, 80(1), 92-113.

Hargittai, E., \& Shafer, S. (2006). Differences in actual and perceived online skills: the role of gender. Social Science Quarterly, 87(2), 432-448.

Harkham Semas, J. (2002). Digital Disconnect: teens say teachers lack Internet skills. District Administration, 38, 11. 
Harris, T., \& Hodges, R. (1995). The literacy dictionary: The vocabulary of reading and writing. Newark, DE: International Reading Association.

Harrison, C., Comber, C., Fisher, T., Haw, K., Lewin, C., \& Lunzer, E. (2003). The impact of information and communication technologies on pupil learning and attainment, ICT in Schools. Coventry, GB: Research and Evaluation Series No. 7, Becta.

Herder, E. (2003). Revisitation Patterns and Disorientation. In Proceedings of the German Workshop on Adaptivity and User Modeling in Interactive Systems (pp. 291 - 294). Hannover: ABIS.

Hobart, M.E., \& Schiffman, Z.S. (2000). Information ages. Literacy, numeracy, and the computer revolution. Baltimore, MD: The John Hopkins University Press.

Hobbs, R. (1997). Expanding the concept of literacy. In R. Kubey (Ed.), Media literacy in the information age (pp. 163-183). New York, NY: Transaction.

Hoem, J., \& Schwebs, T. (2004). Personal Publishing and Media Literacy. World Conference on Computers in Education. Retrieved form http:// infodesign.no/artikler/personal_\%20publishing_media_literacy.pdf

Hoffman, R. (2008). Socioeconomic differences in old age mortality. Dordrecht: Springer.

Holbert, R.L., \& Stephenson, M.T. (2002). Structural equation modeling in the communication sciences, 1995-2000. Human Communication Research, 28, 531-551.

Holborn, M., \& Langley, P. (2004). Sociology: Themes and perspectives. London: Collins Educational.

Horrigan, J.B. (2008). Home Broadband Adoption 2008. Pew Internet \& American Life Project. Retrieved from http://www.pewinternet.org

Hoyle, R.H., \& Panter, A.T. (1995). Writing about structural equation models. In R.H. Hoyle (Ed.), Structural equation modeling (pp. 158-176). Thousand Oaks, CA: Sage.

Hradil, S. (2001). Soziale Ungleichheit in Deutschland. Budrich: UTB.

Hu, L., \& Bentler, P.M. (1999). Cutoff criteria for fit indexes in covariance structure analysis: Conventional criteria versus new alternative. Structural Equation Modeling, 6, 1-55.

Ivanitskaya, L., O’Boyle, I., \& Casey, A.M. (2006). Health information literacy and competencies of information age students: results from the interactive online research readiness self-assessment (RRSA). Journal of Medical Internet Research, 8(2), 2-12

Jackson, L.A., Zhao, Y., Kolenic, A., Fitzgerald, H.E., Harold, R., \& Von Eye, A. (2008). CyberPsychology \& Behavior, 11(4), 437-442. 
Jansen, B.J., \& Pooch, U. (2000).Web user studies: A review and framework for future work. Journal of the American Society of Information Science and Technology, 52(3), 235-246.

Jansen, B.J., \& Spink, A. (2003). An analysis of web information seeking and use: Documents retrieved versus documents viewed. In Proceedings of the $4^{\text {th }}$ International Conference on Internet Computing (pp. 65-69). Las Vegas, Nevada.

Jefferson, G.H. (2005). Malthus, the Industrial revolution, and the Technology Multiplier. Retrieved form http://people.brandeis.edu/ jefferso/

Jenkins, C., Corritore, C.L., \& Wiedenbeck, W. (2003). Patterns of information seeking on the Web: a qualitative study of domain expertise and Web expertise. IT\&Society, 1(3), 64-89.

Jones Thompson, M. (2005). Online Recreation. Technology Review, 108(32).

Jung, J.Y., Qiu, J.L., \& Kim, Y.C. (2001). Internet Connectedness and Inequality: Beyond the Divide. Communication Research, 28(4), 507-35.

Karchmer, R.A. (2001). The Journey Ahead: thirteen teachers report how the Internet influences literacy and literacy instruction in their K-12 classrooms. Reading Research Quarterly, 36(4), 442-466.

Katz, J.E., \& Aspden, P. (1997). A nation of strangers? Communications of the ACM, 40(12), 81-86.

Katz, J.E., \& Rice, R. (2002). Social Consequences of Internet Use: Access, Involvement, and Interaction. Cambridge, MA: MIT Press.

Kellar, M., Hawkey, K., Inkpen, K.M., \& Watters, C. (2008). Challenges of Capturing Natural Web-based User Behaviours. International Journal of Human Computer Interaction, 24(4), 385-409.

Kim, C.W., \& Mauborgne, R. (1999). Strategy, value innovation, and the knowledge economy. Sloan Management Review, 40(3), 41-53.

Klein, D.C.D., Yarnall, L., \& Glaubke, C. (2001). Using technology to assess students' web expertise. Los Angeles, CA: CRESST.

Kling, R. (2000). Learning about information technologies and social change: The contribution of social informatics. The Information Society, 16(3), 217232.

Knobel, M., \& Lankshear, C. (2008). Digital Literacy and participation in online social networking spaces. In C. Lankshear \& M. Knobel (Eds.), Digital Literacies, concepts, policies and practices (pp. 249-278). New York, NY: Peter Lang. 
Kolodinsky, J., Cranwell, M., \& Rowe, E. (2002). Bridging the Generation Gap across the Digital Divide: Teens Teaching Internet Skills to Senior Citizens. Journal of Extension, 40(3).

Kraut, R., Patterson, M., Lundmark, V., Kiesler, S., Mukophadhyay, T., \& Scherlis, W. (1998). Internet Paradox: A Social Technology That Reduces Social Involvement and Psychological Well-Being? American Psychologist, 53(9), 1017-31.

Kuhlemeier, H., \& Hemker, B. (2007). The impact of computer use at home on students' Internet skills. Computers \& Education, 49(2), 460-480.

Kuhlthau, C.C. (1987). Information skills for an information society: A review of research. Syracuse, NY: ERIC Clearinghouse on Information Resources.

Kuiper, E. (2007). Teaching web literacy in primary education. Amsterdam, Vrije Universiteit.

Kuiper, E., Volman, M., \& Terwel, J. (2004). Internet als informatiebron in het onderwijs: Een verkenning van de literatuur. Pedagogische Studiën, 81, 423443.

Kvasny, L. (2006). Cultural (Re)production of digital inequality in a US community technology initiative. Information, Communication \& Society, 9(21), 160-181.

Kwan, M.P. (2001). Cyberspatial cognition and individual access to information: the behavioral foundation of cybergeography. Environment and Planning B: Planning and Design, 28, 21-37.

Langford, L. (1998). Information literacy: a clarification. School Libraries Worldwide, 4(1), 59-72.

Larsson, L. (2002). Digital literacy checklist. Retrieved from http://depts. washington.edu/hserv/teaching/diglit

Lawley, E.L. (1994). The Sociology of Culture in Computer-mediated Communication: An Initial Exploration. Sydney: University of Technology.

Lee, M.J. (2005). Expanding hypertext: Does it address disorientation? Journal of Computer-Mediated Communication, 10(3), article 6.

Levin, D., \& Arafeh, S. (2002). The digital disconnect: the widening gap between Internet-savy students and their schools. Washington DC: Pew Internet \& American Life Project.

Livingstone, S. (2003). The changing nature and uses of media literacy. Retrieved from http://www.lse.ac.uk/collections/media@1se/ 
Livingstone, S., Van Couvering, E., \& Thumim, N. (2005). Adult media literacy: A review of the research literature. Retrieved from http://www.ofcom.org.uk/ consumer_guides/media_literacy

Loos, E. (2010). De oudere: een digitale immigrant in eigen land? Een verkenning naar toegankelijke informatievoorziening. Den Haag: Lemma.

Lorenzen, M. (2001). The land of confusion? High school students and their use of the World Wide Web for research. Research Strategies, 18, 151-163.

Lundt, J.C., \& Vanderpan, T. (2000). It Computes When Young Adolescents Teach Senior Citizens. Middle School Journal, 31(4), 18-22.

Madden, M. (2003). America's online pursuits. Washington, DC: Pew Internet and American Life Project.

Mansell, R. (2002). From Digital Divides to Digital Entitlements in Knowledge Societies. Current Sociology, 50(3), 407-426.

Marchionini, G. (1995). Information seeking in electronic environments. New York: Cambridge University Press.

Marchionini, G., \& White, R. (2007). Find What You Need, Understand What You Find. International Journal of Human-Computer Interaction, 23(3), 205-237.

Markauskaite, L. (2006). Towards an integrated analytical framework of information and communications technology literacy: from intended to implemented and achieved dimensions. Information Research, 11(3), paper 252.87

Markus, L.M. (1987). Toward a "Critical Mass" theory of interactive media. Communication Research, 14(5), 491-511.

Martin, A. (2006). A framework for digital literacy. Glasgow: University of Glasgow.

Martin, J., \& Norman, A.R.D. (1973). The Computerized Society. Armondsworth: Penguin Books Ltd.

Mason, S.M., \& Hacker, K.L. (2003). Applying communication theory to digital divide research. IT\&Society, 1(5), 40-55.

McEuen, S. 2001. How fluent with information technology are our students? Educause Quarterly, 24(4), 8-17.

McClure, C.R. (1994). Network Literacy: a Role for Libraries? Information Technology and Libraries, 13(2), 115-125.

McDonald, S., \& Stevenson, R.J. (1998). Effects of Text Structure and Prior Knowledge of the Learner on Navigation in Hypertext. Human Factors 40(1), 18-27.

McLuhan, M. (1962). The Gutenberg Galaxy. London: Routledge \& Kegan Paul. 
Messaris, P. (1998). Visual aspects of media literacy. Journal of Communication, 48(1), $70-80$.

Messick, S. (1995). Validity of psychological assessment: validation of inferences from persons' responses and performances as scientific inquiry into score meaning. American Psychologist, 50(9), 741-749.

Metzger, M.J., Flanagin, A.J., \& Zwarun, L. (2003). College student web use, perceptions of information credibility, and verification behavior. Computers \& Education, 41(3), 271-290.

Meyrowitz, J. (1998). Multiple media literacies. Journal of communication, 48(1), 96108.

Miall, D.S., \& Dobson, T. (2001). Reading hypertext and the experience of literature. Journal of Digital Information, 2(1), 1-20.

Miller, K. (2006). Organizational Communication, Approaches and processes. Belmont, CA: Thomson Wadsworth.

Mittermeyer, D. (2005). Incoming first year undergraduate students. Education for information, 23(4), 203-32.

Mittman, R., \& Cain, M. (1999). The future of the Internet in health care: A fiveyear forecast. Oakland, CA: California HealthCare Foundation.

Moore, N. (1998). The information society. In Y. Courrier (Ed.), World Information Report (pp. 271-293). Geneva: UNESCO Reference Books.

Morahan-Martin, J.M. (2004). How Internet users find, evaluate, and use online health information: A cross-cultural review. CyberPsychology and Behavior, 7(5), 497-510.

Mossberger, K., Tolbert, C.J., \& Stansbury, M. (2003). Virtual Inequality: Beyond the Digital Divide. Washington, DC: Georgetown University Press.

Mossberger, K., Johns, K., \& King, B. (2006). The digital divide and economic opportunity: Does Internet Use Matter for Less-Skilled Workers. Retrieved from http://www.uic.edu/orgs/stresearch/Documents /

Mizrach, S. (1998). From orality to teleliteracy. Retrieved from http:www.fiu.edu/ $\sim$ mizrachs/orality.htm.

Müller, C. (1999). Networks of personal communities and group communities in different online communication services. In Proceedings of the Exploring Cyber Society: Social, Political, Economic and Cultural Issues (pp. 1-14). University of Northumbria at Newcastle, United Kingdom.

Naito, M. (1991). An information literacy curriculum: a proposal. College Research Libraries News, 52(5), 293-296. 
Naisbitt, J. (1984). Megatrends: Ten new directions transforming our lives. New York: Warner Books.

Nahm, E., \& Resnick, B. (2001). Homebound older adults' experiences with the Internet and email. Computer in Nursing, 19(6), 257-263.

Newhagen, J.E., \& Bucy, E.P. (2004). Routes to media access. In E.P. Bucy \& J.E. Newhagen (Eds.), Media access: Social and psychological dimensions of new technology use (pp. 3-23). London: LEA.

Nielsen, J. (2002). Usability for Senior Citizens. Retrieved from http://www.useit. com/alertbox/20020428.html

Norris, P. (2001). Digital Divide: Civic engagement, information poverty and the Internet worldwide. Cambridge: Cambridge University Press.

Oblinger, D. (2003). Boomers, Gen-Xers and Millennials: understanding the new students. Educause Review, 38(4), 37-47.

Odlyzko, A. (1997). Silicon dreams and silicon bricks: the continuing evolution of libraries. Library Trends, 46(1), 152-167.

O'Hanlon, N.O. (2002). Net knowledge: Performance of new college students on an Internet skills proficiency test. The Internet and Higher Education, 5(1), 55-66.

O'Hara, K. (2004). Curb Cuts on the Information Highway: Older Adults and the Internet. Technical Communication Quarterly, 13(4), 423-445.

Ono, H., \& Zavodny, M. (2003). Gender and the Internet. Social Science Quarterly, 84(1), 111-121.

Otter, M., \& Johnson, H. (2000). Lost in hyperspace: metrics and mental models. Interacting with computers, 13(1), 1-40.

Ozmutlu, S., Spink, A., \& Ozmutlu, H.C. (2004). A day in the life of web searching: an exploratory study. Information Processing and Management: an International Journal, 40(2), 319-345.

Pandolfini, C., Impicciatore, P., \& Bonati, M. (2000) Parents on the Web: risks for quality management of cough in children. Pediatrics, 105, A1-A8.

Park, H.W. (2002). The digital divide in South Korea: Closing and widening divides in the 1990s. Electronic Journal of Communication, 12(1-2).

Park, J., \& Kim, J. (2000). Contextual navigation aids for two world wide web systems. International Journal of Human-Computer Interaction, 12(2), 193-217.

Pask, J.M., \& Saunders, E.S. (2004). Differentiating Information Skills and Computer Skills: A Factor Analytic Approach. Libraries and the Academy, 4(1), 61-73. 
Pieterson, W., \& Ebbers, W. (2008). The use of service channels by citizens in the Netherlands; implications for multi-channel management. International Review of Administrative Sciences, 74(1), 95-110.

Pinkett, R.D. (2000). Bridging the Digital Divide: Sociocultural constructionism and an asset-based approach to community technology and community building. Paper presented at the 81st Annual Meeting of the American Educational Research Association. Retrieved from http://www. media.mit.edu/ rpinkett/papers/aera2000.pdf

Porat, M.U. (1977). The Information Economy: Definition and Measurement. Office of Telecommunications, U.S. Department of Commerce.

Potter, W.J. (2004). Theory of media literacy: A cognitive approach. Thousand Oaks: Sage.

Pritchard, A., \& Cartwright, V. (2004). Transforming that they read: Helping eleven-year-olds engage with Internet information. Literacy, 38(1), 26-31.

Pruulmann-Vengerfeldt, P. (2006). Exploring social theory as a framework for social and cultural measurements of the Information society. The Information Society, 22(5), 303-310.

Quan-Haase, A., Wellman, B., Witte, J., \& Hampton, K.N. (2002). Capitalizing on the Internet: Network capital, participatory capital, and sense of community. In B. Wellman \& C. Haythornthwaite (Eds.), The Internet in everyday life (pp.291-324). Oxford: Blackwell.

Rawls, J. (1971). A Theory of Justice. Cambridge, MA: Harvard University Press.

Redding, C.A., Maddock, J.E., \& Rossi, J.S. (2006). The sequential approach to measurement of health behavior constructs: Issues in selecting and developing measures. Californian Journal of Health Promotion, 4(1), 83-101.

Reeves, B., \& Nass, C. (1996). The media equation: How people treat computers, television, and new media like real people and places. New York, NY: Cambridge University press.

Revere, D. (2005). Digital Literacy Self-Assessment. Retrieved from http://courses. washington.edu/hsstudev/studev/self-assess.html

Robinson, J.P., DiMaggio, P., \& Hargittai, E. (2003). New Social Survey Perspectives on the Digital Divide. IT\& Society, 1(5), 1-22.

Rogers, E.M. (1995). Diffusion of Innovations. New York, NY: Free Press. 
Rothbaum, F., Marthland, N., \& Jannsen, J.B. (2008). Parents' reliance on the Web to find information about children and families: Socio-economic differences in use, skills and satisfaction. Journal of Applied Developmental Psychology, 29(2), 118-128.

Rury, J.L. (2005). Education and social change: themes in the history of American schooling. Mahwah, NJ: Lawrence Erlbaum Associates, Inc.

Sabel, C.F., \& Zeitlin, J. (1985). Historical alternatives to mass production: politics, markets, and technology in nineteenth century industrialization. Past and Present, 108, 133-76.

Salomon, G. (1977). Effects of encouraging Israeli mothers to co-observe sesame street with their five-year-olds. Child Development, 48(3), 1146-51.

Scheufele, D.A., \& Nisbet, M.C. (2002). Being a citizen online. New opportunities and dead ends. The Harvard International Journal of Press/Politics, 7(3), 55-75.

Schumacher, P., \& Morahan-Martin, J. (2001). Gender, Internet and computer attitudes and experiences. Computers in Human Behavior, 17(1), 95-110.

Schumacker, R.E., \& Lomax, R.G. (2004). A beginner's guide to structural equation modeling. Mahwah, NJ: L. Erlbaum Associates.

Shaw, A.C. (1995). Social constructionism and the inner city: Designing environments for social development and urban renewal. Cambridge, MA: MIT Media laboratory.

Shneiderman, B., Byrd, D., \& Croft, B. (1997). Clarifying search: a user-interface framework for text searches. D-Lib Magazine. Retrieved from http:// www.dlib.org/dlib/january97/retrieval/01 shneiderman.html

Selwyn, N (2006). Digital division or digital decision? A study of non-users and low-users of computers. Poetics, 34(4-5), 273-292.

Sen, A.K. (1985). Commodities and Capabilities. Oxford: Oxford University Press.

Serveas, J. (2003). The European information society: A reality check. Bristol: Intellect Books.

Shapiro, J., \& Hughes, S. (1996). Information technology as a liberal art. Educom Review, 31(2), 31-36.

Shenton, A.K., \& Dixon, P. (2003). A comparison of youngsters' use of CD$\mathrm{ROM}$ and the Internet as information resources. Journal of the American Society for Information Science and Technology, 54(11), 1029-49.

Shepperd, S., Charnock, D., \& Gann, B. (1999). Helping patients access high quality health information. British Medical Journal, 319(7212), 764-766.

Silverblatt, A. (1995). Media literacy: Keys to interpreting media messages. Westport, CT: Preager. 
Slouka, M. (1995). War of the worlds: Cyberspace and the high-tech assault on reality. New York: Basic Books.

Søby, M. (2003). Digital competences: From ICT skills to digital bildung. Oslo: University of Oslo.

Somekh, B., Lewin, C., Mavers, D., Fisher, T., Harrison,C., \& Haw, K. (2002). ImpaCT2: Pupils' and teachers' perceptions of ICT in the home, school and community. Coventry, GB: Becta.

Stevens, J. (1996). Applied Multivariate Statistics for the Social Sciences. Mahwah, New Jersey: Lawrence Erlbaum Publishers.

Steyaert, J. (2002). Inequality and the digital divide: myths and realities. In S. Hick \& J. McNutt (Eds.), Advocacy, activism and the internet (pp. 199-211). Chicago: Lyceum Press.

Stone, L. (1969). Literacy and Education in England 1640-1900. Past \& Present, 42, 69-139.

Susskind, A.M., Bonn, M.A., \& Dev, C. (2003). To look or book: An examination of consumers' apprehensiveness toward internet use. Journal of Travel Research, 41 (3), 256-264.

Sutherland-Smith, W. (2002). Weaving the literacy web: Changes in reading from page to screen. Reading Teacher, 55(7), 662-669.

Talja, S. (2005). The social and discursive construction of computing skills. Journal of the American Society for Information Science and Technology, 56(1), 13-22.

Tambini, D. (2000). Universal Internet access: A realistic view. Retrieved from http://www.csls.ox.ac.uk/

Tesdell, L.S. (2005). Evaluating Web-sources: Internet literacy and L2 academic writing. Technical Communication, 52, 401.

Thatcher, A. (2008). Web search strategies: The influence of Web experience and task type. Information Processing and Management, 44(3), 1308-13.

Tichenor, P.J., Donohue, G.A., \& Olien, C.N. (1970). Mass media flow and differential growth in knowledge. Public Opinion Quarterly, 34(2), 159-170.

Tilly, C. (1999). Durable Inequality. University of California Press.

Thoreau, E. (2006). Ouch!: An examination of the self-representation of disabled people on the Internet. Journal of Computer-Mediated Communication, 11(2), article 3 .

Torkzadeh, G., \& Van Dyke, T. (2002). Effects of training on Internet selfefficacy and computer user attitudes. Computers in Human Behavior, 18(5), 479-494. 
Tyner, K. (1998). Literacy in a Digital World. Mahwah NJ: Lawrence Erlbaum Associates.

University of Valencia (2000). Computer Skills Self-Assessment Survey. Retrieved form http://www.unm.edu/ unmvc/Busntech/ComputerAssessment Survey.htm

Valkenburg, P.M., Peter, J., \& Schouten, A.P. (2006). Friend networking sites and their relationship to adolescents' well-being and social self-esteem. CyberPsychology \&o Behavior, 9(5), 584-590.

Van Damme, M., De Haan, J., Kraan, K. et al. (2005). Verzonken Technologie. Ict en de arbeidsmarkt. Den Haag: SCP

Van Deursen, A. (2007). Where to go in the near future: Diverging perspectives on online public service delivery. Lecture Notes in Computer Science, 4656, 143-154.

Van Deursen, A., \& Gui, M. (2008). Digital skills in the information society. Working paper. Enschede: University of Twente.

Van Deursen, A., \& Pieterson, W. (2006). The Internet as a service channel in the Public Sector. A substitute or complement of traditional service channels? Paper presented at the International Communication Association Conference 2006. Retrieved from http://doc.utwente.nl/59807/1/Deursen 06internet.pdf

Van Deursen, A.J.A.M., \& Van Dijk, J.A.G.M. (2009). Trendreport: Motivation, Access, Use and Skills. A European and Dutch perspective. Enschede: University of Twente.

Van Deursen, A.J.A.M., \& Van Dijk, J.A.G.M. (2010). Civil servants' Internet skills: are they ready for e-government? Lecture Notes in Computer Science, $6228,130-143$.

Van Deursen, A., Van Dijk, J., \& Ebbers, W. (2006). Why E-government usage lags behind: Explaining the gap between potential and actual usage of electronic public services in the Netherlands. Lecture Notes in Computer Science, 4084, 269-280.

Van Dijk, J. (1999). The network society: Social aspects of new media. London: Sage Publications.

Van Dijk, J. (2005). The deepening divide. Inequality in the information society. London: Sage Publications.

Van Dijk, J. (2006). Digital divide research, achievements and shortcomings. Poetics, 34(4-5), 221-35. 
Van Dijk, J., \& Hacker, K. (2003). The Digital Divide as a complex and dynamic phenomenon. The Information Society, 19(4), 315-327.

Van Dijk, L., De Haan, J., \& Rijken, S. (2000). Digitalisering van de leefwereld; een onderzoek naar Informatie- en Communicatietechnologie en sociale ongelijkheid. Den Haag: SCP

Van Duivenboden, H.P.M., Lips, A.M.B., \& Korsten, A.F.A. (2001). Klantgericht werken en ICT. Op weg naar vraaggerichte elektronische publieke dienstverlening. Bestuurskunde, 11(8), 322-327.

Van Ingen, E., De Haan, J., \& Duimel, M. (2007). Achterstand en afstand. Digitale vaardigheden van lager opgeleiden, ouderen, allochtonen en inactieven. Den Haag: SCP.

Virkus, S. (2003). Information literacy in Europe: a literature review. Information Research, 8(4), paper no. 159.

Volman, M., Van Eck, E., Heemskerk, I., \& Kuiper, E. (2005). New technologies, new differences. Gender and ethnic differences in pupils' use of ICT in primary and secondary education. Computers \& Education, 45(1), 35-55.

Wales, R.J. (2004). A person issue before a technology issue. Paper presented at the HCI and the Older Population Workshop. Leeds, UK. Retrieved from http:// www.dcs.gla.ac.uk/utopia/workshop/wales.pdf

Wallace, R., Kupperman, J., Krajcik, J., \& Soloway, E. (2000). Science on the Web: Students online in a sixth-grade classroom. Journal of the Learning Sciences, 9(1), 75-104.

Walraven, A., Brand-Gruwel, S., \& Boshuizen, H.P.A. (2008). Informationproblem solving: a review of problems students encounter and instructional solutions. Computers in Human Behavior, 24(3), 623-48.

Wang, L., Bretschneider, S., \& Gant, J. (2005). Evaluating Web-Based eGovernment services with a citizen-centric approach. Paper presented at the 38th Annual Hawaii International Conference on Systems Sciences. Big Island, Hawaii.

Warschauer, M. (2002). Reconceptualising the digital divide. First Monday, 7(7).

Warschauer, M. (2003). Technology and Social inclusion: Rethinking the Digital Divide. Cambridge, MA: The MIT Press.

Wasserman, I.M., \& Richmond-Abbott, M. (2005). Gender and the Internet: Causes of variation in access, level, and scope of use. Social Science Quarterly, 86(1), 252-270.

Weber, M. (1978). Economy and Society. Berkeley: University of California Press. 
Webster, F. (2002). The Information society revisited. In: Lievrouw, L.A. \& Livingstone, S. (Eds.). Handbook of New Media (pp. 443-457). London: Sage Publications.

Webster, J., \& Ahuja, J.S. (2006). Enhancing the design of web navigation systems: The influence of user disorientation on engagement and performance. Management Information Systems Quarterly, 30(3), 661-678.

Weil, L.D., \& Rosen, M.M. (1995). The psychological impact of technology from a global perspective: a Study of technological sophistication and computer anxiety in university students from twenty-three countries. Computers in Human Behavior, 11(1), 95-133.

Wellman, B. (2001). Physical place and cyberspace: The rise of personalized networks. International Urban and Regional Research, 25(2), 227-252.

Willis, S., \& Tranter, B. (2006). Beyond the digital divide. Internet diffusion and inequality in Australia. Journal of Sociology, 42(1), 43-59.

Wilson, E.J. (2006). The Information revolution and developing countries. Cambridge, MA: MIT Press.

Witte, J.C., \& Mannon, S.E. (2009). The Internet and social inequalities. New York: Routledge.

Zauberman, G. (2003). The intertemporal dynamics of consumer lock-in. Journal of Consumer Research, 30(3), 405-419.

Zellweger, P. (1989). Scripted Documents: A hypermedia path mechanism. In Proceedings of the Hypertext'89 (pp. 1-14). Baltimore, ACM.

Zillien, N., \& Hargittai, E. (2009). Digital Distinction: Status-Specific Types of Internet Usage. Social Science Quarterly, 90(2), 274-291.

Zurkowski, P.G. (1974). The information service environment: Relationships and Priorities. Washington, DC: National Commission on Libraries and Information Science. 


\section{Appendix I Assignments used in performance test 1}

\begin{tabular}{|c|c|}
\hline Operational Internet skills & Assignment 1 (max time allowed: 12 minutes) \\
\hline $\begin{array}{l}\text { Opening Web sites by entering the URL in the } \\
\text { browser's location bar; }\end{array}$ & $\begin{array}{l}\text { 1. Go to the Web site of the Dutch Tax and Custom } \\
\text { Administration (www.belastingdienst.nl) }\end{array}$ \\
\hline \multirow[t]{3}{*}{ Using text or images with hyperlinks; } & $\begin{array}{l}\text { 2. Click on the link 'Download and order' in the menu } \\
\text { on the right. }\end{array}$ \\
\hline & Click on the subject 'Marriage' \\
\hline & $\begin{array}{l}\text { Click on the link to the brochure 'When you are } \\
\text { getting married'. }\end{array}$ \\
\hline Opening various common file formats (e.g., & 3. Open the brochure 'When you are getting married'. \\
\hline & $\begin{array}{l}\text { Save the brochure in the folder 'Marriage' on the } \\
\text { desktop of the computer. }\end{array}$ \\
\hline Navigating forward and backward between & 4. Use the back-button to go back to the 'Download \\
\hline pages using the browser buttons; & $\begin{array}{l}\text { and order' page. Click on the link 'Declaration 2006' } \\
\text { placed in column 'private'. Click on 'Declaration } \\
\text { software } 2006 \text { (Windows)' }\end{array}$ \\
\hline Saving files on the Hard Disk; & $\begin{array}{l}\text { 5. Save the file 'Electronic declaration IB } 2006 \text { for } \\
\text { Windows' on the desktop. }\end{array}$ \\
\hline Bookmarking Web sites; & $\begin{array}{l}\text { 6. Go back to the homepage of the Dutch Tax } \\
\text { Administration. Add the homepage to the favourites } \\
\text { (or bookmarker). }\end{array}$ \\
\hline Operating Internet-based search engines: & 7. Use the search engine on top of the Web site \\
\hline Entering keywords in the proper field; & using the keyword 'save-as-you-earn deduction' \\
\hline Executing the search operation; & Open the third search result of the search \\
\hline Opening search results in the search result lis & \\
\hline
\end{tabular}

Operating Internet-based forms:

Using the different types of fields and buttons; Submitting a form.

Assignment 2 (max time allowed: 8 minutes) 8. Go to the Child care allowance Web site of the Dutch Tax and Custom administration: www.toeslagen.nl/reken/kinderopvangtoeslag/ Complete the fields using the information given.

\begin{tabular}{l}
\hline Formal Internet skills \\
Not becoming disoriented when navigating \\
web site; \\
Not becoming disoriented when navigating \\
between Web sites;
\end{tabular}
Assignment 3 (max time allowed: 10 minutes) 1. Go to the Web site of the Central Office of Information, postbus51.nl. Follow the options Accommodation / Rent / Rental price/ Rent Subsidy. Choose the option: 'What is rent subsidy and how do I apply for it?'

2. Click on the link 'Applying for rent subsidy'. Go to the homepage of the Allowance Web site in the new window. Go back to the homepage of Postbus51 in the old window.

Not becoming disoriented when opening and browsing through search results;
3. Perform a search on the Postbus 51 Web site with keyword 'rental price'. Open the first search result. Open the second search result.

Navigating on the Internet by using hyperlinks Assignment 4 (max time allowed: 10 minutes) (e.g., menu links, textual links, image links) in 4. Imagine that you just moved to Nijmegen. You would like to look up the physical office addresses of different menu and Web site layouts. the following organisations: IB-Groep, UWV and CWI. 


\begin{tabular}{ll}
\hline Information Internet skills & Assignment 5 (max time allowed: 12 minutes) \\
\hline Locating required information, by: & 1. Imagine that you just moved to Rotterdam. You \\
Defining search options or queries; & decide to buy a subscription to a parking lot. Find out \\
Selecting information (on a Web site); & how much a subscription to the car park named \\
Evaluate information found. & 'Spaanse Kade' costs. Use the homepage of the \\
& municipality of Rotterdam (www.rotterdam.nl).
\end{tabular}

Locating required information, by:

Choosing a Web site or a search system to

seek information;

Defining search options or queries;

Selecting information (in search results);

Evaluating information sources.

Assignment 6 (max time allowed: 12 minutes) 2. Imagine that, during a day at the shopping mall, your passport is stolen. Use a search engine (e.g., www.google.nl or the one you use at home) to find out what type of document you need to apply for a new passport after the old one is stolen.

Locating required information, by:

Choosing a Web site or a search system to seek information;

Defining search options or queries;

Selecting information (in search results);

Evaluating information sources.

Assignment 7 (max time allowed: 12 minutes)

3. Imagine that you are 25 years old. In between September 1st and December 30th you had a fulltime job in a factory (40 hours / week). Your wage was 1275 euro gross every month. This was not much. Use a search engine (e.g., www.google.nl or the one you use at home) to find out whether you were entitled to a higher salary during this period. (Yes, because the salary was lower than _ euro. / No, because the salary was higher than _ euro)

\begin{tabular}{l}
\hline Strategic Internet skills \\
\hline Taking advantage of the Internet, by: \\
Developing an orientation towards the goal; \\
Taking the right action to reach this goal; \\
Making the right decision to reach this goal; \\
Gaining the benefits resulting from this goal.
\end{tabular}
Assignment 8 (max time allowed: 12 minutes) 1. When your employer paid you too little, what financial recourse do you have can you then personally obtain? Sort this out using the Internet.

Taking advantage of the Internet, by: Developing an orientation towards a particular goal;

Taking the right action to reach this goal; Making the right decision to reach this goal; Gaining the benefits resulting from this goal.

Assignment 8 (max time allowed: 30 minutes) 2. Image that there are national elections soon. You are in doubt whether to vote for the PvdA, the CDA or the VVD. You have the following opinions:

- You are in favour of using nuclear energy;

- You are in favour of a high child care allowance;

- You are against having two nationalities.

Using the Internet, find out which of these three political parties have your first, second and third preference. 


\section{Appendix II Assignments used in performance test 2}

\begin{tabular}{|c|c|}
\hline Operational Internet skills & Assignment 1 (max time allowed: 6 minutes) \\
\hline $\begin{array}{l}\text { Opening Web sites by entering the URL in the } \\
\text { browser's location bar: }\end{array}$ & 1. Go to the Web site of the CBR (www.cbr.nl). \\
\hline Using text or images with hyperlinks; & $\begin{array}{l}\text { 2. Click on the link 'Motor' in the menu on the left. } \\
\text { Click on the subject 'Motor and scooter', placed in } \\
\text { the column 'Brochures.' }\end{array}$ \\
\hline Opening various common file formats; & 3. Open the brochure 'Motor and scooter.' \\
\hline Saving files on the Hard Disk; & $\begin{array}{l}\text { Save the brochure in the folder 'CBR' in My } \\
\text { Documents. }\end{array}$ \\
\hline $\begin{array}{l}\text { Navigating forward and backward between } \\
\text { pages using the browser buttons; } \\
\text { Bookmarking Web sites; }\end{array}$ & $\begin{array}{l}\text { 4. Use the back button to go back to homepage of } \\
\text { the CBR Web site. } \\
5 \text {. Add the homepage to the Favourites (or } \\
\text { bookmarks }\end{array}$ \\
\hline
\end{tabular}

Operating Internet-based forms:

Using the different types of fields and buttons;

Submitting a form.

Operating Internet-based search engines:

Entering keywords in the proper field;

Executing the search operation;

Opening search results in the search result lists.

Saving files from the Internet on the Hard Disk;

Assignment 2 (max time allowed: 5 minutes)

6. Go to the Web site of Marktplaats

(www.marktplaats.nl).

Click on the link 'Uitgebreid zoeken.'

Complete the fields using the information given.

7. Execute the search function and open the third search result.

\section{Formal Internet skills \\ Not becoming disoriented when navigating within a Web site; \\ Not becoming disoriented when navigating between Web sites;}

Not becoming disoriented when opening and browsing through search results;

Navigating on the Internet by using hyperlinks (e.g., menu links, textual links, image links) in different menu and Web site layouts.
8. Save the logo of Markplaats in the upper left corner on the desktop of the computer Assignment 3 (max time allowed: 8 minutes) 1. Go to the Web site of the ANWB (www.anwb.nl). Follow the options Car / Sell / Selling Occasion. Choose the option: 'Selling my car via Auto Trader.'.

Choose the option: 'What is rent subsidy?' 2. In both windows, go to the homepage of the Web site opened: Go to the homepage of the Autotrader Web site in the new window. Go to the homepage of the ANWB in the old window. 3. Perform a search on the ANWB Web site with keyword 'beach'. Open the first search result. Open the second search result.

Assignment 4 (max time allowed: 10 minutes) 4. Find the addresses of the following three Museums in The Hague. Use the museums' Web site: Het Mauritshuis (www.mauritshuis.nl/), Het Museon (www.museon.nl/), and Het Letterkundig Museum (www.letterkundigmuseum.nl). 


\begin{tabular}{ll}
\hline Information Internet skills & Assignment 5 (max time allowed: 12 minutes) \\
\hline Locating required information, by: & 1. Imagine... 8 months ago you subscribed to a \\
Defining search options or queries; & mobile telephone contract with T-mobile. Now you \\
Selecting information (on a Web site); & would like to take advantage of the new T-mobile \\
Evaluate information found. & iPhone offer. Answer the following question, using \\
& the T-mobile Web site (www.t-mobile.nl). Is it \\
& possible to subscribe to a T-mobile iPhone contract \\
& as a continuation of your current subscription?
\end{tabular}

Locating required information, by:

Choosing a Web site or a search system to

seek information;

Defining search options or queries;

Selecting information (in search results);

Evaluating information sources.

Locating required information, by:

Choosing a Web site or a search system to

seek information;

Defining search options or queries;

Selecting information (in search results);

Evaluating information sources.

Assignment 6 (max time allowed: 12 minutes) 2. Imagine... You would like to go out for a luxury dinner in Amsterdam next week. You prefer the restaurant with the most Michelin stars. Answer the following question using a search engine (e.g., Google or the Web site you use at home): What restaurant would you go to?

Assignment 7 (max time allowed: 12 minutes) 3. Imagine... You bought a rubber boat of 9.84 feet in length. You can reach a maximum speed of 17 $\mathrm{mph}$ with this boat. Answer the following question using a search engine (e.g., Google or the Web site you use at home): Are you allowed to use this boat in public waters like the Maas?

\begin{tabular}{|c|c|}
\hline Strategic Internet skills & Assignment 8 (max time allowed: 15 minutes) \\
\hline $\begin{array}{l}\text { Taking advantage of the Internet, by: } \\
\text { Developing an orientation towards a particular } \\
\text { goal; } \\
\text { Taking the right action to reach this goal; } \\
\text { Making the right decision to reach this goal; } \\
\text { Gaining the benefits resulting from this goal. }\end{array}$ & $\begin{array}{l}\text { 1. Imagine... You, your partner and your son go on } \\
\text { a trip to Amsterdam. Going by car costs } 4 \text { Euros for } \\
\text { every } 12.4 \text { miles, excluding } 4 \text { hours of parking in } \\
\text { central Amsterdam. Your son has a train pass } \\
\text { (which might also offer discounts for you) and you } \\
\text { wonder if the train would be a cheaper option. Use } \\
\text { the Internet to find out which option is cheaper. }\end{array}$ \\
\hline $\begin{array}{l}\text { Taking advantage of the Internet, by: } \\
\text { Developing an orientation towards a particular } \\
\text { goal; } \\
\text { Taking the right action to reach this goal; } \\
\text { Making the right decision to reach this goal; } \\
\text { Gaining the benefits resulting from this goal. }\end{array}$ & $\begin{array}{l}\text { Assignment } 9 \text { (max time allowed: } 30 \text { minutes) } \\
2 \text {. Imagine... from March the } 7 \text { th through the } 13 \text { th } \\
\text { you are going on a trip to London with your partner. } \\
\text { You would like to book two plane tickets from a } \\
\text { nearby airport and a hotel in central London. Find } \\
\text { out how much this would cost using the Internet, } \\
\text { aiming to identify the cheapest options. Consider } \\
\text { flight, hotel and travel expenses to and from the } \\
\text { airport in London. }\end{array}$ \\
\hline
\end{tabular}




\section{Appendix III Assignments used in performance test 3}

\begin{tabular}{|c|c|}
\hline Operational Internet skills & Assignment 1 (max time allowed: 12 minutes) \\
\hline $\begin{array}{l}\text { Opening Web sites by entering the URL in } \\
\text { the browser's location bar; }\end{array}$ & 1. Go to the Web site of the RIVM (www.rivm.nl). \\
\hline Using text or images with hyperlinks; & $\begin{array}{l}\text { 2. Click on the link 'Infectieziekten' in the menu on } \\
\text { the left. Click on the subject 'Thema's'. Click on the } \\
\text { subject 'Hoofdluis'. Click on the subject } \\
\text { 'Voorlichtingsmateriaal downloaden of bestellen' }\end{array}$ \\
\hline $\begin{array}{l}\text { Opening various common file formats; } \\
\text { Saving files on the Hard Disk; } \\
\text { Navigating forward and backward } \\
\text { between pages using the browser buttons; } \\
\text { Bookmarking Web sites. }\end{array}$ & $\begin{array}{l}\text { 3. Open the brochure 'Brochure veelgestelde vragen' } \\
\text { Save the brochure in "My Documents". } \\
\text { 4. Use the back button to go back to homepage of } \\
\text { the RIVM Web site. } \\
\text { 5. Add the homepage to the Favourites (or } \\
\text { bookmarks }\end{array}$ \\
\hline & Assignment 2 (max time allowed: 8 minutes) \\
\hline Operating Internet-based forms: & $\begin{array}{l}\text { 6. Go to the Web site of MinVMS (www.minvws.nl). } \\
\text { Click on the link 'Uitgebreid zoeken.' }\end{array}$ \\
\hline $\begin{array}{l}\text { Using the different types of fields and } \\
\text { buttons; } \\
\text { Submitting a form. }\end{array}$ & $\begin{array}{l}\text { Complete the fields using the information given. } \\
\text { 7. Execute the search function and open the third } \\
\text { search result. }\end{array}$ \\
\hline $\begin{array}{l}\text { Saving files from the Internet on the Hard } \\
\text { Disk. }\end{array}$ & $\begin{array}{l}\text { 8. Save the logo of the MinVWS on the desktop of } \\
\text { the computer. }\end{array}$ \\
\hline Formal Internet skills & Assignment 3 (max time allowed: 10 minutes) \\
\hline $\begin{array}{l}\text { Not becoming disoriented when navigating } \\
\text { within a Web site; }\end{array}$ & $\begin{array}{l}\text { 1. Go to the website of ZonMW (www.zonmw.nl). } \\
\text { Follow the options Onderwerpen / Jeugd / Zorg } \\
\text { Choose the option: 'RIVM/Jeugdgezondheid'. }\end{array}$ \\
\hline $\begin{array}{l}\text { Not becoming disoriented when navigating } \\
\text { between Web sites; }\end{array}$ & $\begin{array}{l}\text { 2. In both windows, go to the homepage of the Web } \\
\text { site opened: } \\
\text { Go to the homepage of the RIVM Web site in the new } \\
\text { opened window. } \\
\text { Go to the homepage of the RIVM website in the } \\
\text { original window. }\end{array}$ \\
\hline $\begin{array}{l}\text { Not becoming disoriented when opening } \\
\text { and browsing through search results. }\end{array}$ & $\begin{array}{l}\text { 3. Perform a search on the ZonMW Web site with } \\
\text { keyword 'infectie'. Open the first search result. } \\
\text { Open the fourth search result. }\end{array}$ \\
\hline & Assignment 4 (max time allowed: 10 minutes) \\
\hline $\begin{array}{l}\text { Navigating on the Internet by using } \\
\text { hyperlinks (e.g., menu links, textual links, } \\
\text { image links) in different menu and Web } \\
\text { site layouts. }\end{array}$ & $\begin{array}{l}\text { 4. Find the addresses of the following three health } \\
\text { organizations. Use the Web sites of the organization: } \\
\text { ISALA Clinic in Zwolle (www.isala.nl). } \\
\text { BOSK Organization for the disabled (www.bosk.nl/). } \\
\text { GGZ Enschede (www.ggznederland.nl) }\end{array}$ \\
\hline
\end{tabular}




\begin{tabular}{ll}
\hline Information Internet skills & Assignment 5 (max time allowed: 12 minutes) \\
\hline Locating required information, by: & 1. Imagine... You would like to know more about the \\
Defining search options or queries; & H1N1 influenza. This was originally called the Swine \\
Selecting information (on a Web site); & Flu. Answer the following question, using the Web \\
Evaluate information found. & $\begin{array}{l}\text { site of Dokterdokter (www.dokterdokter.nl): Why is } \\
\text { the name Swine flu not correct? }\end{array}$
\end{tabular}

Locating required information, by: Choosing a Web site or a search system to seek information;

Defining search options or queries; Selecting information (in search results); Evaluating information sources.

Locating required information, by:

Choosing a Web site or a search system to seek information;

Defining search options or queries;

Selecting information (in search results); Evaluating information sources.
Assignment 6 (max time allowed: 12 minutes) 2. Imagine... During a hike you are bitten by a tick. A red spot appears that increases. This is a sign you have been infected with Lyme borreliosis. A friend recommends to start with an antiviral (remedy against viral infections) immediately., since Lyme's disease can have very unpleasant consequences, especially when treatment starts too late! Answer the following question using a search engine (e.g., Google or the Web site you use at home): Is it a good idea to start an antiviral remedy?

Assignment 7 (max time allowed: 12 minutes) 3. Imagine... The last few months, your son has been suffering problems with his back. His back shows a deviation to the left. It looks like one leg is shorter than the other, although this not appear to be the case. Answer the following question using a search engine (e.g., Google or the Web site you use at home): What is the name of the condition your son suffers from?

\begin{tabular}{|c|c|}
\hline Strategic Internet skills & Assignment 8 (max time allowed: 12 minutes) \\
\hline $\begin{array}{l}\text { Taking advantage of the Internet, by: } \\
\text { Developing an orientation towards a } \\
\text { particular goal; } \\
\text { Taking the right action to reach this goal; } \\
\text { Making the right decision to reach this } \\
\text { goal; } \\
\text { Gaining the benefits resulting from this } \\
\text { goal. }\end{array}$ & $\begin{array}{l}\text { 1. Imagine... You have a three year old son. Your } \\
\text { mother gives you the advise to give him extra } \\
\text { vitamins A and D. Your mother believes these are } \\
\text { necessary for a healthy growth. Answer the following } \\
\text { question using a search engine (e.g., Google or the } \\
\text { Web site you use at home): Would you give your son } \\
\text { both extra vitamins A and D? }\end{array}$ \\
\hline $\begin{array}{l}\text { Taking advantage of the Internet, by: } \\
\text { Developing an orientation towards a } \\
\text { particular goal; } \\
\text { Taking the right action to reach this goal; } \\
\text { Making the right decision to reach this } \\
\text { goal; } \\
\text { Gaining the benefits resulting from this } \\
\text { goal. }\end{array}$ & $\begin{array}{l}\text { Assignment } 9 \text { (max time allowed: } 30 \text { minutes) } \\
\text { 2. Imagine... Your mother is } 82 \text { years old. Lately, she } \\
\text { has been suffering from dementia and impaired } \\
\text { hearing. You decided to find a homecare } \\
\text { organization in Enschede that has a special caring } \\
\text { program for these complaints. You also would like the } \\
\text { organization to organize daily activities for seniors. } \\
\text { Use the Internet to find a homecare organization in } \\
\text { Enschede that meets your demands? }\end{array}$ \\
\hline
\end{tabular}



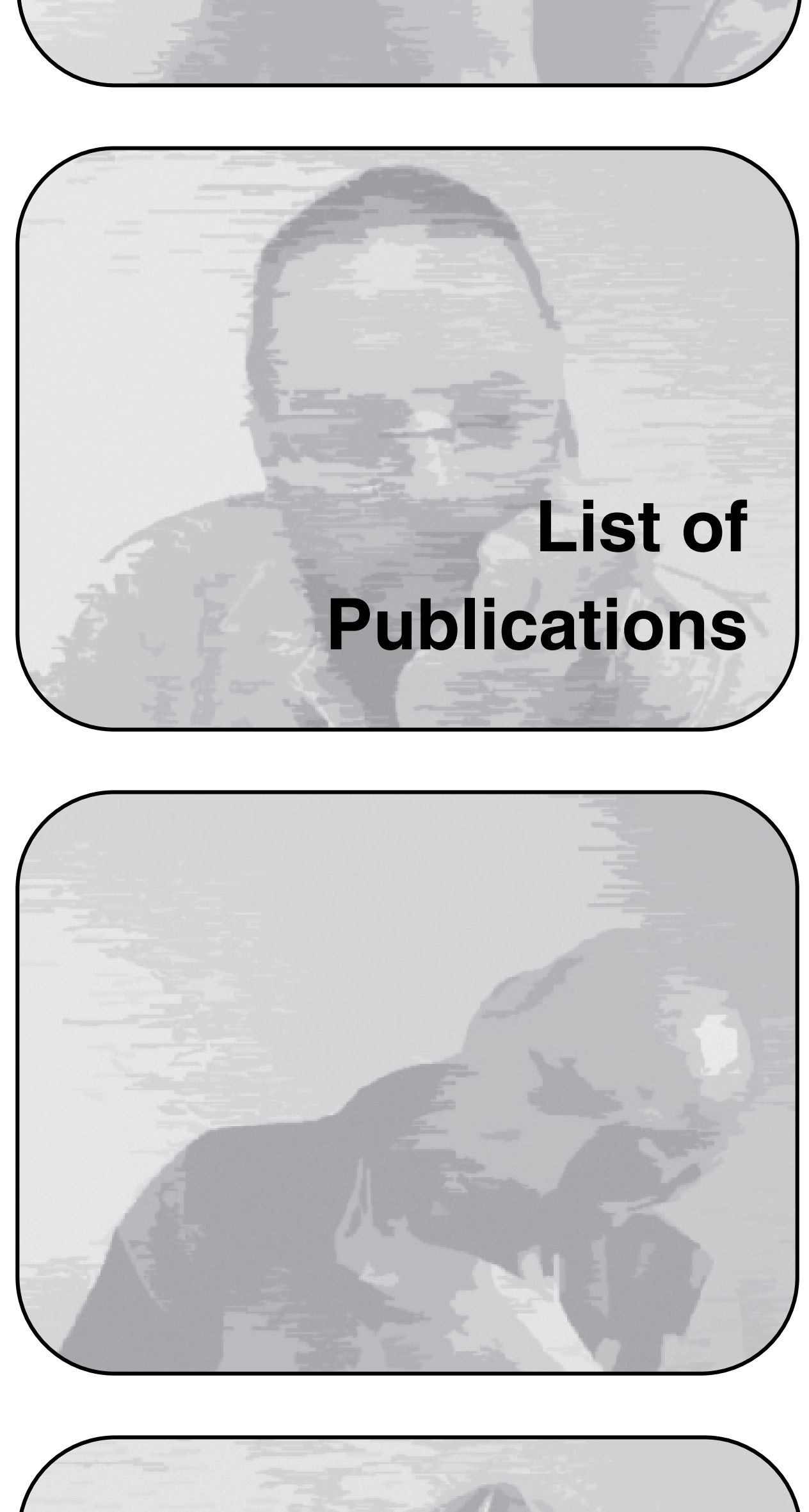


\section{Journal articles}

Van Deursen, A.J.A.M., \& Van Dijk, J.A.G.M. (submitted). Internet skills, vital resources in an information society.

Van Deursen, A.J.A.M., \& Van Dijk, J.A.G.M. (submitted). Digital media skills and traditional media Skills, is there much of a difference?

Van Deursen, A.J.A.M., \& Van Dijk, J.A.G.M. (submitted). Internet Skills Performance Tests: Are People Ready For eHealth?

Van Deursen, A.J.A.M., Van Dijk, J.A.G.M., \& Peters, O. (in press). Rethinking Internet skills. The contribution of gender, age, education, Internet experience, and hours online to medium- and content-related Internet skills. Poetics

Van Deursen, A.J.A.M., \& Van Dijk, J.A.G.M. (in press). Internet skills and the digital divide. New Media and Society

Van Deursen, A.J.A.M., \& Van Dijk, J.A.G.M. (2010). Measuring Internet skills. International Journal of Human-Computer Interaction, 26(10), 891-916.

Van Deursen, A., \& Van Dijk, J.A.G.M. (2010). Internet skills and civil servants: Are they ready for eGovernment? Lecture Notes in Computer Science, 4656, 143-154.

Van Deursen, A.J.A.M., \& Van Dijk, J.A.G.M. (2009). Using the Internet: Skillrelated problems in users' online behavior. Interacting with Computers, 21(56), 393-402.

Van Deursen, A.J.A.M., \& Van Dijk, J.A.G.M. (2009). Improving digital skills for the use of online public information and services. Government Information Quarterly, 26(2), 333-340.

Van Deursen, A. (2007). Where to go in the near future: Diverging perspectives on online public service delivery. Lecture Notes in Computer Science, 4656, 143-154.

Van Deursen, A., Van Dijk, J., \& Ebbers, W. (2006). Why e-government usage lags behind: Explaining the gap between potential and actual usage of electronic public services in the Netherlands. Lecture Notes in Computer Science, 4084, 269-280. 


\section{Conference papers}

Van Deursen, A.J.A.M., Van Der Vaart, R., Drossaert, C., Van Dijk, J., Taal, E., \& Van De Laar, M. (2010). Validation of a Dutch Version of the EHealth Literacy Scale (eHEALS). Poster presented at the Medicine $2.0{ }^{\circledR}$ conference 29-30 November.

Van Deursen, A.J.A.M., \& Van Dijk, J.A.G.M. (2010). The Older the better. Rethinking Internet skills. Presented at the ICA Conference in Singapore, 22-26 June. Awarded: TOP Faculty paper

Van Deursen, A.J.A.M., \& Van Dijk, J.A.G.M. (2010). New media skills and traditional media skills. Is there much of a difference? Presented at the ICA Conference in Singapore, 22-26 June.

Van Deursen, A.J.A.M., \& Van Dijk, J.A.G.M. (2010). Internet Skills: Participating in an information society. Presented at the Etmaal van de Communicatiewetenschap conference at the University of Gent in Belgium, 4-5 February.

Van Deursen, A. (2009). Internet skills, vital resources in contemporary society. Presented at the Participating in a mediated world conference organized by the Platform for Communication, Media, and Information (CMI) within the Royal Netherlands Academy of Arts and Sciences, 27 November.

Van Deursen, A.J.A.M. (2008). Internet skills in an information society. Presented at the fifth Annual Work Conference of the Netherlands Institute of Government (NIG) in Enschede, 20-21 November.

Van Deursen, A.J.A.M., \& Van Dijk, J.A.G.M. (2008). Measuring Internet skills. Performance tests of operational, formal, information and strategic Internet skills among the Dutch population. Presented at the EGOV 2008 conference in Krakow, 4-8 September.

\section{Runner up: Best practical concept.}

Van Deursen, A.J.A.M., \& Van Dijk, J.A.G.M. (2008). Measuring Internet skills. Performance tests of operational, formal, information and strategic Internet skills among the Dutch population. Presented at the ICA Conference in Montreal, 22-26 May. 


\section{Book chapters}

Van Dijk, J., \& Van Deursen, A. (2009). Inequalities of Digital Skills and How to Overcome Them. In: E. Ferro, Y. K. Dwivedi, R. Gil-Garcia and M. D. Williams (Eds.), Handbook of Research on Overcoming Digital Divides: Constructing an Equitable and Competitive Information Society. Hershey, IGI Global.

\section{Research Reports}

Van Deursen, A.J.A.M., \& Van Dijk, J.A.G.M. (2010). Trendrapport Computer-en Internetgebruik 2010. Een Nederlands en Europees perspectief. Enschede: Universiteit Twente.

Van Deursen, A.J.A.M., \& Van Dijk, J.A.G.M. (2009). Trendrapport 2009: Motivatie, Toegang, Gebruik en Vaardigheden. Een Europees en Nederlands perspectief. Enschede: Universiteit Twente.

Van Deursen, A.J.A.M., \& Van Dijk, J.A.G.M. (2009). eV aardigheden en eAwareness van Nederlandse ambtenaren. Enschede: Universiteit Twente.

Van Deursen, A.J.A.M., \& Van Dijk, J.A.G.M. (2008). Digitale vaardigheden van Nederlandse burgers. Een prestatiemeting van operationele, formele, informatie en strategische vaardigheden bij het gebruik van overheidswebsites. Enschede: Universiteit Twente.

Van Deursen, A.J.A.M., Van Dijk, J.A.G.M., \& Boland, D. (2007). Elektronische publieke dienstverlening in de toekomst. Enschede: Universiteit Twente.

Van Deursen, A.J.A.M., \& Van Dijk, J.A.G.M. (2006). Van Aanbod naar V raag, Tijd voor een Perspectiefwisseling: Verkennend Onderzoek naar het Gebruik van Elektronische Overbeidsdiensten door Burgers in Nederland. Enschede: Universiteit Twente. 

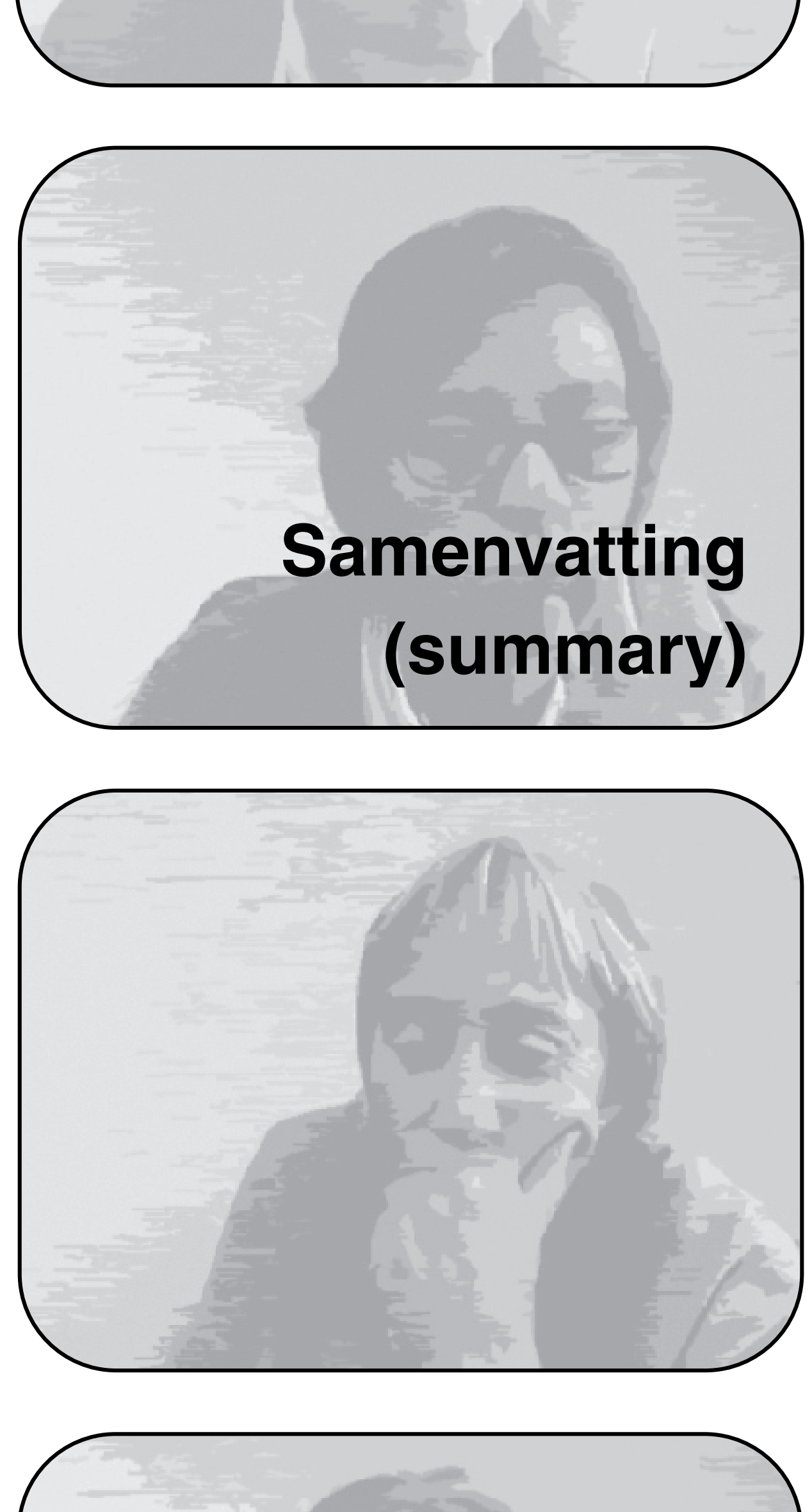
In de huidige samenleving vormen digitale communicatietechnologieën zoals computers en het Internet een belangrijk onderdeel van het dagelijks leven. Het gebruik van deze technologieën is echter niet altijd even vanzelfsprekend. Wanneer men de historische ontwikkeling van communicatietechnologieën bekijkt is dit niet zo verwonderlijk. De introductie van het schrift, het alfabet, de drukpers en audiovisuele media stelden stuk voor stuk nieuwe eisen aan een reeds bestaand pakket van benodigde vaardigheden waarover de gebruikers moesten beschikken. Een zorgwekkende trend is dat de consequenties van het niet kunnen gebruiken van de nieuwe technologieën steeds groter zijn. Bij de inmiddels traditionele communicatietechnologieën was het eerst slechts een kleine groep die hiervan gebruik maakten (denk bijvoorbeeld aan de monniken die het lezen en schrijven beheersten in de middeleeuwen). Van het merendeel van de bevolking werd niet verwacht dat zij deze technologieën al beheersten en directe nadelige gevolgen van het niet (kunnen) gebruiken bleven uit. In hoofdstuk 1 wordt betoogd dat dit nu niet meer het geval is. Speciale aandacht gaat uit naar dé communicatietechnologie die de afgelopen jaren haar stempel heeft gedrukt op de informatiesamenleving; het Internet. De transformatie van informatie en diensten naar het Internet gaat gestaag door, waarbij de veronderstelling lijkt te zijn dat deze vervolgens algemeen toegankelijk zijn. De rol die het Internet speelt in het dagelijks leven hangt af van de vaardigheden die nodig zijn om deze communicatietechnologie te gebruiken. Het zijn deze vaardigheden die centraal staan in dit proefschrift.

Het niet kunnen voldoen aan de toenemende eisen die de ontwikkeling van nieuwe communicatietechnologieën met zich mee brengt en de nadelige gevolgen die hieruit voortvloeien staan centraal in Hoofdstuk 2. In dit hoofdstuk worden de nadelige gevolgen van het niet beschikken over de vaardigheden om het Internet te gebruiken bekeken vanuit het perspectief van sociale ongelijkheid. Een gangbare classificatie voor sociale ongelijkheid is door Bourdieu voorgesteld. Hij onderscheidt economisch kapitaal (geld en materiële eigendommen), cultureel kapitaal (de kennis, vaardigheden en onderwijs die iemand een hogere status geven) en sociaal kapitaal (relaties en sociale ondersteuning). In hoofdstuk 2 wordt betoogd dat deze kapitaalvormen een recursief verband hebben met het Internet. Om te beginnen zijn zij samen een vereiste voor Internettoegang. Economisch kapitaal is nodig voor de aanschaf van een computer en Internetaansluiting, sociaal kapitaal is nodig om verbinding met het Internet te maken en het vervolgens te gebruiken, en cultureel kapitaal is nodig om wegwijs te worden in de enorme hoeveelheid informatie die het Internet vertegenwoordigt. Wanneer aan 
deze eisen is voldaan kan er vervolgens kapitaalversterking optreden. De gebruiker kan economisch kapitaal bijvoorbeeld vergroten door het goedkoper aanschaffen van producten of het vinden van een betere baan. Sociaal kapitaal kan vergroot worden door het uitbreiden van het fysieke netwerk naar een virtueel netwerk waardoor betrokkenheid en gemeenschapsgevoel worden vermeerderd. Cultureel kapitaal ten slotte kan vergroot worden door bijvoorbeeld het Internet te benutten om kennis te vergaren. In hoofdstuk 2 wordt betoogd dat het bij het verband tussen kapitaalvormen en het Internet van belang is dat het gebruik van het Internet wordt aangepast aan de wensen van de gebruikers waardoor de nietgebruikers steeds meer op achterstand worden gezet. Zij zullen in toenemende mate negatieve gevolgen ondervinden. Hierbij kan gedacht worden aan volgeboekte vluchten, uitverkochte concerten, vergeven banen of misgelopen potentiële partners. Het idee van ongelijkheid dat wordt versterkt door nieuwe technologieën ligt ten grondslag aan de digitale kloof. Oorspronkelijke conceptualisaties van de digitale kloof beschouwden deze vooral als een tweedeling op basis van fysieke toegang tot computers en het Internet. Recente conceptualisaties benadrukken dat ook sociale, psychologische en culturele aspecten in ogenschouw moeten worden genomen wanneer het gaat over het wel of geen toegang hebben tot een nieuwe technologie. Een belangrijke variabele die een rol speelt in deze conceptualisaties is de Internetvaardigheid.

Er valt heel wat af te dingen op reeds bestaande metingen van Internetvaardigheden. Meestal wordt er gebruik gemaakt van enquêtes waarin indirect naar vaardigheden wordt gevraagd of waarin mensen een schatting moeten maken van hun eigen vaardigheden. Tevens wordt er dikwijls een definitie van Internetvaardigheden gehanteerd die zich beperkt tot zogenaamde knoppenkennis. Een literatuuroverzicht in hoofdstuk 3 leert dat er een grote verscheidenheid aan Internetvaardigheden gerelateerde termen bestaat, maar dat er weinig overeenstemming is over wat deze precies betekenen. Tevens ontbreken operationele definities. Op basis van bestaande concepten en gerelateerde onderzoeksstromen wordt er een definitie voor Internetvaardigheden voorgesteld. Hierin worden vier soorten vaardigheden onderscheiden die samen beschouwd kunnen worden als vereiste voor regulier Internetgebruik. In de definitie wordt onderscheid gemaakt tussen vaardigheden om het Internet te kunnen bedienen (zogenaamde mediumgerelateerde vaardigheden die bestaan uit operationele en formele vaardigheden) en inhoudelijk gerelateerde vaardigheden (informatie en strategisch). Hiermee wordt een technologisch-deterministische visie vermeden. 
De definitie heeft een sequentieel en conditioneel karakter: zonder mediumgerelateerde vaardigheden komt men niet toe aan het in de praktijk brengen van de inhoudelijke vaardigheden. Verder wordt vooral het actieve karakter van Internetgebruik benadrukt, dat groter is in vergelijking met de relatief passieve traditionele media door de grotere mogelijkheden tot interactie. De definitie luidt:

Operationele vaardigheden zijn het kunnen...

- $\quad$...bedienen van een Internet browser:

- Het openen van websites door de URL in de adresbalk te typen,

- Het vooruit en terug navigeren door gebruik te maken van de browser knoppen,

- Het opslaan van bestanden op de harde schijf,

- Verschillende formaten kunnen openen en opslaan (bijvoorbeeld PDF),

- Het opslaan van websites in de Favorieten.

- ...bedienen van een zoekmachine op het internet:

- Het invullen van zoekwoorden in een zoekveld,

- Het uitvoeren van een zoekopdracht,

- Het openen van zoekresultaten.

- ...gebruiken van online formulieren:

- Gebruik maken van de verschillende typen invoervelden en knoppen (bijvoorbeeld dropdown menu's),

○ Het versturen van een formulier.

Formele vaardigheden zijn het kunnen...

- $\quad$...navigeren op het internet door:

- Hyperlinks te gebruiken (in een menu, in een tekst, in plaatjes etc.) in verschillende menu en website lay-outs.

- ...behouden van een gevoel van oriëntatie tijdens het navigeren op het internet:

- Niet gedesoriënteerd raken binnen een website,

○ Niet gedesoriënteerd raken tijdens het surfen tussen websites,

- Niet gedesoriënteerd raken tijdens het openen van en surfen tussen zoekresultaten.

Informatievaardigheden zijn het kunnen...

- $\quad$...vinden en gebruiken van informatie, door:

- Het kiezen van een geschikt zoeksysteem (of plaats om informatie te zoeken),

- Het definiëren van zoekwoorden die zich op het informatieprobleem richten,

- Het selecteren van geschikte informatiebronnen,

- Het evalueren van informatiebronnen.

Strategische vaardigheden zijn het...

- $\quad$...voordeel behalen uit Internetgebruik, door:

○ Het oriënteren op een bepaald doel,

- De juiste actie ondernemen om het doel te behalen,

- De juiste beslissingen nemen om het doel te behalen,

- De voordelen van dit doel behalen. 
Om een betrouwbaar en volledig beeld te krijgen van het niveau van de Internetvaardigheden van de Nederlandse bevolking zijn er in 2007, 2008 en 2009 prestatiemetingen uitgevoerd. Deze prestatiemetingen worden besproken in hoofdstuk 4. Deelnemende proefpersonen zijn geworven met een gestratificeerde en gerandomiseerde steekproef waardoor de respondenten systematisch verschilden in geslacht, leeftijd en opleiding. In totaal moest elke deelnemer negen opdrachten uitvoeren met behulp van het Internet, twee om operationele vaardigheden te meten, twee om formele vaardigheden te meten, drie om informatie vaardigheden te meten en twee om strategische vaardigheden te meten. Succesvolle voltooiing en de tijd die men nodig had voor de opdrachten zijn als maat voor Internetvaardigheid gebruikt. De eerste prestatiemeting (2007) gebruikte opdrachten in de context van de overheid. Omdat deze specifieke context de resultaten betreffende Internetvaardigheden zou kunnen vertekenen, zijn deze vaardigheden opnieuw aan een toetsing onderworpen in een tweede (2008) en derde (2009) prestatiemeting, waarbij vergelijkbare opdrachten geformuleerd werden in de context van respectievelijk algemeen vermaak en gezondheid. De resultaten van de drie prestatiemetingen kwamen overeen. Een groot aantal opdrachten werd niet succesvol voltooid. Van de vier typen vaardigheden waren de operationele en formele vaardigheden niet het meest problematisch. De informatie- en strategische vaardigheden daarentegen werden door een groot deel van de proefpersonen niet voldoende beheerst om de taken succesvol af te ronden. Operationele en formele Internetvaardigheden bleken een noodzakelijke maar onvoldoende voorwaarde voor het in de praktijk brengen van de informatie en strategische Internetvaardigheden.

Om variabalen te identificeren die bijdragen aan het niveau van internetvaardigheden zijn de resultaten van de drie prestatiemetingen apart geanalyseerd. Aangezien de structuur en type taken in elke studie gelijkwaardig waren zijn de resultaten van de drie studies ook samengevoegd om multivariate analyses uit te kunnen voeren. De variabele die de sterkste voorspeller is van de vier Internetvaardigheden is het opleidingsniveau. Opleidingsniveau draagt bij aan zowel de mediumgerelateerde operationele en formele vaardigheden als aan de inhoudelijke informatie- en strategische vaardigheden. Omdat de prestaties op de mediumgerelateerde vaardigheden ook sterk bepalend zijn voor de prestaties op de inhoudelijke vaardigheden is er naast het directe effect op de inhoudelijke vaardigheden ook een indirect effect waarneembaar. Naast opleidingsniveau speelt ook leeftijd een belangrijke rol. Jongeren worden veelal geassocieerd met frequent 
en vaardig Internetgebruik. Ouderen daarentegen worden beschouwd als problematische en onzekere gebruikers. Leeftijd vertoont inderdaad een negatieve relatie met het niveau van de operationele en formele vaardigheden. Jongeren presteren hierop veel beter dan ouderen. Echter, het niveau van de inhoudelijke vaardigheden bij jongeren is precair. Leeftijd vertoont een direct positief effect op de inhoudelijke informatie- en strategische vaardigheden wat impliceert dat naarmate mensen ouder zijn, hun informatie- en strategische vaardigheden ook groter zijn. Helaas wordt dit positieve effect tenietgedaan door een gebrek aan mediumgerelateerde vaardigheden. Met andere woorden, ouderen worden zodanig door hun operationele en formele vaardigheden gehinderd dat zij op inhoudelijke vaardigheden slechter scoren dan jongeren. De veronderstelling dat het vaardigheidsprobleem vanzelf wordt opgelost met het uitsterven van de huidige oudere generatie is dus onjuist. Dit blijkt ook uit het gegeven dat de factor Internetervaring weinig bijdraagt aan het vaardigheidsniveau, met uitzondering van een effect op de operationele Internetvaardigheden. Daarnaast nemen de informatie- en strategische vaardigheden niet toe door hoeveelheid gebruik. Het hebben gevolgd van een Internetcursus, het hebben van sociale ondersteuning, de locatie van Internetgebruik en de socio-economische positie spelen allen slechts een marginale rol bij het beschikken over Internetvaardigheden.

De specifieke vaardigheidsproblemen die mensen ondervinden tijdens het gebruik van het Internet en de factoren die hier aan bijdragen staan centraal in hoofdstuk 5. Op basis van de in hoofdstuk 3 voorgestelde definitie en de uitvoering van de opdrachten is er een codeerschema opgesteld waarmee mogelijke en ondervonden problemen kunnen worden geïdentificeerd. De in de eerste prestatiemeting softwarematig opgenomen schermacties zijn geanalyseerd. Voorbeelden van problemen betreffende operationele Internetvaardigheden zijn het niet in staat zijn om een PDF bestand op te slaan of te openen en het missen van de basisvaardigheden voor het gebruik van een zoekmachine. Zoekwoorden werden bijvoorbeeld door enkele senioren aan elkaar getypt of in de adresbalk geplaatst. Een veelvoorkomend formeel vaardigheidsprobleem is het gebrek aan oriëntatie tijdens het surfen. Bij het openen van een nieuw venster bijvoorbeeld, zijn het vooral ouderen die het oorspronkelijke venster niet meer terugvinden, ook al is het duidelijk zichtbaar op de achtergrond. Verder veroorzaakten bepaalde menuontwerpen (vooral pop-up menu's) problemen bij ouderen en lager opgeleiden. Het feit dat elke website een eigen ontwerp en menustructuur hanteert maakt het er voor veel mensen niet makkelijker op. Betreffende de 
informatievaardigheden bleek dat proefpersonen uit alle groepen problemen ondervonden in het informatiezoekproces: ze hebben bijvoorbeeld veel moeite met het definiëren van geschikte zoekwoorden (vaak veel te algemeen) en met het selecteren van geschikte zoekresultaten (waarbij vaak niet verder wordt gekeken dan de eerste drie resultaten, laat staan verder dan de eerste zoekpagina). Opmerkelijk is ook de bevinding dat niemand gevonden informatie lijkt te evalueren. Dat wat op het Internet staat wordt te gemakkelijk voor waar aangenomen. Bij de strategische vaardigheden viel ten slotte op dat beslissingsondersteunende websites vaak voor verkeerde doelen worden ingezet. Tevens werken lager en middelbaar opgeleide proefpersonen vaak ongestructureerd (willekeurig browsen) en ondervinden zij regelmatig problemen met het gefocust blijven op het voorgenomen doel.

In dit onderzoek is aangetoond dat er een grote groep mensen is met een laag niveau van Internetvaardigheden. Hierdoor worden deze mensen steeds meer benadeeld. De exacte invloed van de Internetvaardigheden op bijvoorbeeld het dagelijkse leven of op sociale ongelijkheid kan echter pas worden vastgesteld wanneer deze vaardigheden als onafhankelijke variabelen worden opgenomen in grootschalig onderzoek. Omdat het uitvoeren van prestatiemetingen erg tijdrovend en duur is, zijn er in hoofdstuk 6 zogenaamde proxyvariabelen geïdentificeerd die in vragenlijsten gebruikt kunnen worden voor het meten van Internetvaardigheden. Daartoe hebben proefpersonen in de tweede en derde prestatiemeting achteraf een vragenlijst gekregen met daarin aan vaardigheden gerelateerde stellingen. In de vragenlijst bij de tweede prestatiemeting zijn stellingen met een maat voor frequentie gebruikt en in de vragenlijst bij de derde prestatiemeting zijn stellingen met een maat voor overeenkomst gebruikt. De frequentiestellingen vertoonden de hoogste correlatie met de daadwerkelijke prestaties. Verdere analyse op validiteit en betrouwbaarheid leidde uiteindelijk tot een instrument met daarin 23 stellingen. Deze stellingen vormden samen echter nog geen sluitend geheel en worden op het moment van schrijven verder getoetst in grootschaliger onderzoek. Ter afsluiting van hoofdstuk 6 worden de problemen die het pionierswerk van instrumentontwikkeling voor Internetvaardigheden opleverde verder bediscussieerd.

In hoofdstuk 7 passeren de meest in het oog springende bevindingen en hun implicaties de revue. Centraal staat het gemeten vaardigheidsniveau, de ongelijkmatige verdeling hiervan binnen verschillende bevolkingssegmenten en de 
betekenis hiervan in de huidige informatiesamenleving. Onder andere wordt de eerder vermelde migratie naar het Internet (vaak ter vervanging van traditionele vormen van informatievoorziening en dienstverlening) geëvalueerd. Betoogd wordt dat er een reële kans bestaat dat grote delen van de bevolking worden uitgesloten van belangrijke informatie en diensten. Vervolgens wordt gekeken wat er nieuw is aan deze vorm van uitsluiting. De belangrijke rol van opleidingsniveau bijvoorbeeld, is immers ook bij traditionele media waarneembaar. Twee verschillen staan centraal. Ten eerste zijn er bovenop de vaardigheden van het lezen en schrijven een reeks extra mediumgerelateerde vaardigheden nodig die samen een nieuwe barrière opwerpen. Ten tweede verschillen bepaalde communicatiecapaciteiten van het Internet behoorlijk van die van traditionele media waardoor er hogere eisen worden gesteld aan de inhoudelijke informatie en strategische vaardigheden. Dit betekent, zo wordt aannemelijk gemaakt, dat bestaande ongelijkheden die veroorzaakt worden door verschillen in gebruik van traditionele media versterkt. Deze versterkte vorm van ongelijkheid kan als onrechtvaardig worden beschouwd. Hoofdstuk 7 eindigt met een discussie betreffende de methodiek en de resultaten. Tevens worden suggesties voor mogelijk vervolgonderzoek gegeven.

De digitale kloof bestond in zijn oorspronkelijk vorm uit een tweedeling van fysieke toegang, de 'haves' en de 'have-nots'. Dit maakte beleid dat moest leiden tot vermindering van de kloof relatief eenvoudig; het beschikbaar stellen van een computer met Internetaansluiting was voldoende. $\mathrm{Nu}$ is beargumenteerd dat Internetvaardigheden een prominentere plaats in moeten nemen in onderzoek naar de digitale kloof - zij zijn ten slotte 'vital assets in an information society' wordt het dichten ervan veel complexer. Ter afsluiting worden in hoofdstuk 8 twee mogelijke strategieën besproken om het probleem van een tekort aan Internetvaardigheden aan te pakken. De eerste strategie richt zich op de toegankelijkheid en bruikbaarheid van websites. Hier worden vanuit het oogpunt van elk van de vier vaardigheden aanbevelingen gedaan voor het ontwerp van een website. Voorbeelden zijn het op websites aanbieden van duidelijke terugkoppeling (bijvoorbeeld dat een zoekmachine nog aan het zoeken is) of het hanteren van consistente en eenvoudige ontwerpen ten behoeve van operationele vaardigheden. Een meer rigoureuze aanpak voor bijvoorbeeld de gezamenlijke overheidsinstanties is het aanbieden van een alternatieve websitevariant die qua ontwerp en didactische aanpak voor elke instantie standaard is. Op een laag niveau van informatievaardigheden zouden websites in kunnen spelen door bijvoorbeeld 
suggesties te geven bij het definiëren van zoekwoorden of door andere opties te suggereren bij het niet vinden van zoekresultaten ("bedoelde u misschien"). Vanuit het oogpunt van de strategische vaardigheden is het aanbevelenswaardig beslissingsondersteunende software te ontwikkelen voor onderwerpen waarmee mensen vaak in aanraking komen. Deze software dient dan te worden toegespitst op het keuzegedrag van mensen. De tweede strategie richt zich op het verbeteren van Internetvaardigheden zelf. Hierbij is er onderscheid gemaakt tussen verschillende levensfasen. Voorbeelden zijn het geven van speciale aandacht aan informatievaardigheden in het onderwijs door deze te integreren in bestaande vakken, het toespitsen van het ICT-cursuspakket voor werknemers op het gebruik van het Internet (vooral in informatieberoepen), of het aanbieden van cursussen in wijkwerk en club- en buurthuizen. Openbare bibliotheken kunnen de centra bij uitstek worden waar volwassenen hun informatievaardigheden kunnen vergroten. Voor ouderen kunnen passende cursussen in operationele en formele computeren internetvaardigheden beschikbaar gemaakt worden. Wanneer zij deze vaardigheden beter beheersen, zullen ze beter scoren dan de jongeren op de inhoudelijke vaardigheden. 

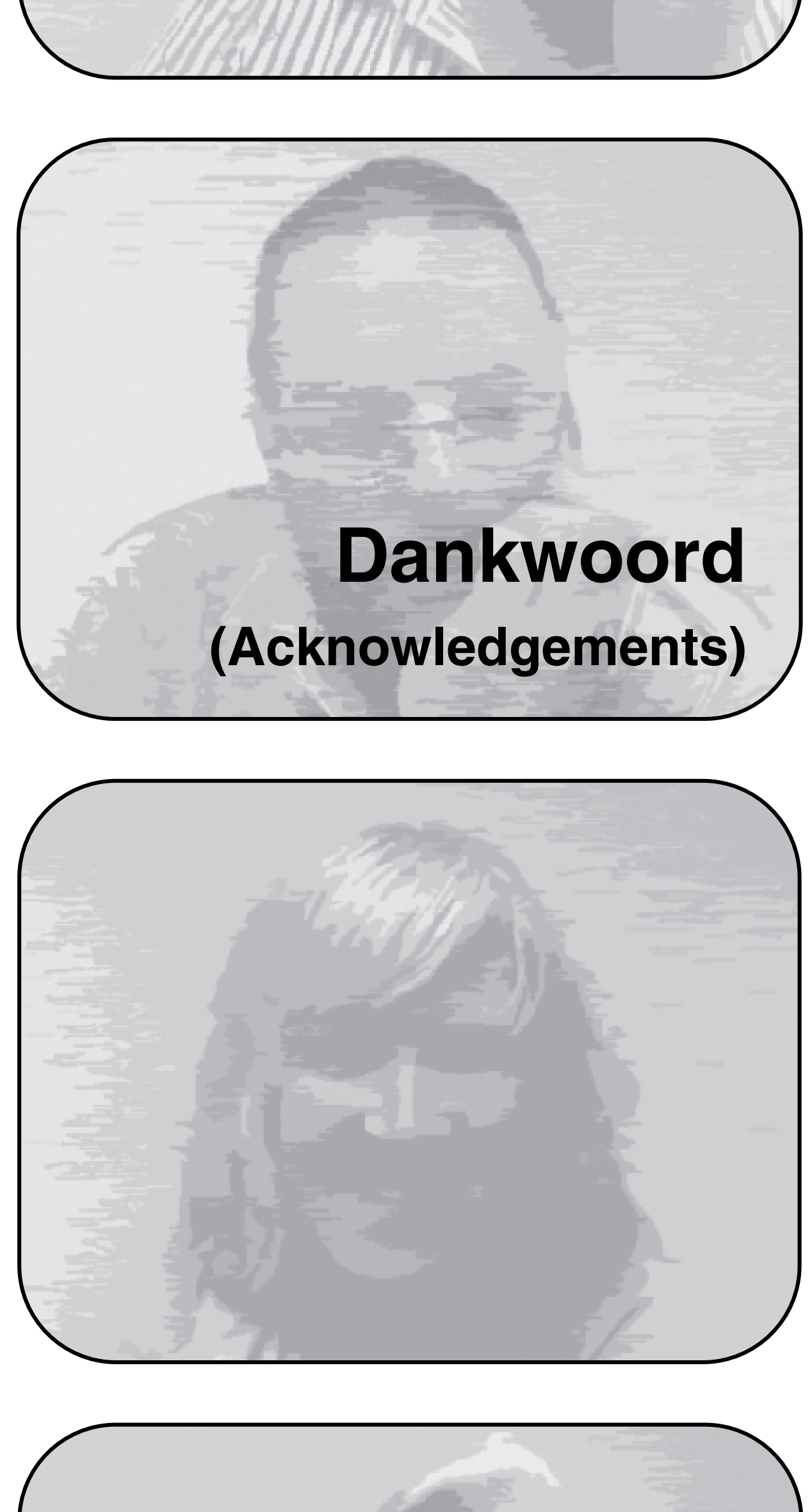
Als iemand mij voor mijn 19e levensjaar vroeg wat ik wilde worden, antwoordde ik steevast 'etholoog' (in de vroegste jaren sprak ik over 'beestjes onderzoeken'). Uren kon ik mijzelf vermaken met het liggen naast een mierenhoop of het aanschouwen van een aquariumvis. Op basis van mijn huidige baan zou je kunnen zeggen dat ik er destijds behoorlijk naast zat, maar dit is niet het geval. Ik bedoel natuurlijk niet dat ik uren voor een aquarium zit, maar wel dat ik in mijn baan als promovendus geregeld het gedrag van dieren bestudeer, de wat complexere soort zeg maar. Wanneer een homo sapiens zich achter een computer met internetverbinding nestelt, vinden de meest dierlijke gedragingen plaats (van paringsrituelen tot foerageren). Geregeld heb ik mijzelf afgevraagd of wij wel zo veel verder zijn ontwikkeld dan een gemiddeld dier uit de klasse der mammalia. Dat dit wel het geval is laat ik nu zien door het uitspreken van een dankwoord zonder lik in het gezicht of uitgebreide vlooibeurt.

Laat ik beginnen met mijn promotor, een bijzonder man zonder wiens inbreng dit proefschrift er zeker niet had gelegen. Jan, ik kan me je eerste college in de mastertrack "Nieuwe media en communicatietechnologie" nog goed herinneren. "Wat een tof onderzoek doet die man in Hawaïblouse met bijpassende Powerpoint schermen" was mijn eerste gedachte. $\mathrm{Nu}$, een aantal jaren later doe ik zelf volop mee in de fascinerende wetenschap van de informatiesamenleving. De maatschappelijke relevantie van mijn onderzoek en de spraakmakende resultaten, zorgen samen dat ik met genoegen terug kijk op mijn tijd als promovendus. Jan, ik ben je buitengewoon dankbaar dat je me het vertrouwen, de kans en de vrijheid hebt gegeven dit te realiseren. Ik hoop de komende jaren nog veel van je te leren en de plezierige samenwerking nog lang voort te zetten. Er staat ons nog een boel werk te wachten, of zoals je het zelf poëtisch formuleert: "we gaan ons te pletter schrijven".

Een tweede woord van dank gaat uit naar Oscar. Ondanks dat het pas laat vast stond dat je assistent-promotor werd, heb ik erg veel aan je gehad. Complexe multivariate analyses en andere statistische gekkigheden, voor jou was niets te gortig. En het mooie is dat dit naast een gemoedsrustige Alexander ook nog eens een mooie plak op heeft geleverd!

Dan zijn er nog een aantal andere collega's die een stempel hebben gedrukt op deze dissertatie. Is het niet door het stellen van geïnteresseerde vragen, dan wel door het meedenken over de materie. Ook niet onbetekenend zijn de vele gezellige momenten die zich afspeelden op en buiten de campus. Dat mijn eigenwijze houding tijdens de pubquiz de pret nooit heeft weten te drukken is reden genoeg voor groot respect mijnerzijds. De volgende personen wil ik even bij 
naam noemen: Bob, Claartje, Fenne, Jeroen, Joost, Jordy, Joris, Jurjen, Karin, Lex, Lidwien, Loes, Marieke, Nalini, Nicol(e), Peter, Renske, Sanne, Somaya, Suzanne, Thea, Thomas, Vanessa en Willem, bedankt voor de stimulerende werkomgeving en de vele leuke momenten!

I am very grateful to my committee members. Thank you for your time and effort in reading my dissertation.

Bij deze wil ik ook de contactpersonen bij de verschillende organisaties die op enig moment hebben meegewerkt aan de in deze dissertatie genoemde onderzoeken bedanken.

$\mathrm{Na}$ de promotor, assistent-promotor, collega's en betrokken zakenrelaties volgen gewoonlijk nog een hele riedel personen waarvan de precieze inbreng aan het proefschrift vaak raadselachtig is. Dit zijn in de regel familieleden en vrienden. Laat ik beginnen met de eerste categorie. Als mijn (stief)ouders, (stief)zussen en stiefbroers me in de afgelopen vier jaar niet zo vaak succes hadden gewenst met mijn 'afstuderen', dan had dit boekje er nooit gelegen! Het is fijn om zowel in het zuiden (twee) en in het noorden (één) des lands thuisfronten te hebben waarvan je weet dat je er altijd welkom bent.

Dan rest er nog een stel vrienden die een belangrijke rol spelen in de momenten van vermaak. Altijd goed jullie weer te zien en een biertje te drinken. Hopelijk hoef ik nadat jullie het proefschrift hebben gelezen - want dat doen jullie natuurlijk - nooit meer uit te leggen dat promoveren meer is dan een luie ambtenarenbaan met als centraal thema vakantie. Speciale aandacht gaat uit naar twee vrienden die mij tijdens de verdediging bijstonden: Bram, die ondanks het beginnend vaderschap en de bijkomende hectiek direct klaar stond, en Jordy, de meest geroutineerde paranimf die er bestaat. Het is goed jullie in de buurt te hebben.

Naast een promotietraject bracht Universiteit Twente me via de Batavierenrace nog iets moois, mijn Marijke. Relativeren wordt door deze slimme, mooie en vooral nuchtere Friezin een stuk eenvoudiger. Bedankt voor je geduld en je rotsvaste vertrouwen.

Houdoe en bedankt! 